\title{
NOVEL APPROACHES TO MODELING AND TREATMENT OF COGNITIVE DEFICITS IN NEUROPSYCHIATRIC DISEASES
}

\author{
PhD Thesis \\ Dissertation \\ for the award of the degree \\ "Doctor of Philosophy" (Ph.D.) \\ Division of Mathematics and Natural Sciences \\ of the Georg-August-University Göttingen \\ Faculty of Biology
}

submitted by

AHMED EL-KORDI

born in

CAIRO, EGYPT

Göttingen 2010 


\section{MEMBERS OF THE THESIS COMMITTEE}

Prof. Dr. Dr. Hannelore Ehrenreich

Division of Clinical Neuroscience

Max Planck Institute of Experimental Medicine

Göttingen

Prof. Dr. Nils Brose

Department of Molecular Neurobiology

Max Planck Institute of Experimental Medicine

Göttingen

Prof. Dr. Henning Gibbons

Institute of Medical Psychology

Georg-August-University

Göttingen

Date of the oral examination:

08.10.2010 


\section{DECLARATION}

I hereby declare that this submission is my own work and that, to the best of my knowledge and belief, it contains no materials previously published or written by another person, nor material which to a substantial extent has been accepted for the award of any other degree of the university or other institute of higher education, except where due acknowledgement has been made in the text. 


\section{ACKNOWLEDGEMENTS}

First of all, I would like to express my deepest gratitude to Prof. H. Ehrenreich for supporting me throughout my $\mathrm{PhD}$ years and for giving me the great opportunity to work on the here mentioned projects. Furthermore, I would like to thank her for her patience, unparalleled supervision and enthusiasm in each and every respect.

I owe many thanks to the members of my thesis committee, Prof. Brose and Prof. Gibbons for their scientific support during my PhD period.

I want also to mention my gratitude to the behavioral team of the Division of Clinical Neuroscience: Dr. Konstantin Radyushkin, Dr. Bartosz Adamcio, Anja Ronnenberg and Martesa Tantra for their collegiality, cooperation and great scientific help.

Of course I want to extend my thanks to all members of the Division of Clinical Neuroscience for their support and help. Many thanks go especially to our lab assistants Swetlana Sperling and Kathrin Hannke. Additionally, I want to thank Dr. Derya Sargin, for the great time we spent on working on the transgenic project and for the inspiring discussions.

I want to thank my family for their boundless and unconditional support all over the years of my studies. 


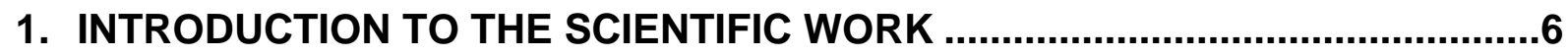

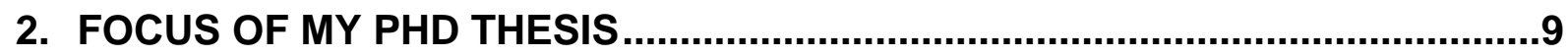

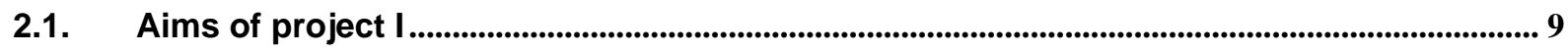

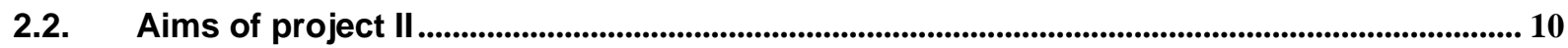

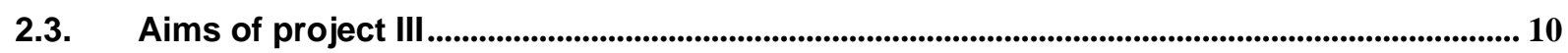

3. AN EXPERIMENTAL APPROACH COMBINING GENETIC AND ENVIRONMENTAL FACTORS FOR INDUCING A SCHIZOPHRENIA-LIKE PHENOTYPE IN MICE............................................................................... 11

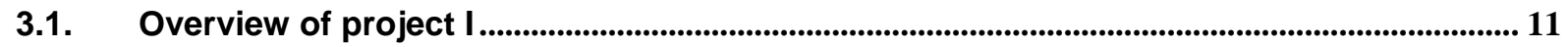

3.2. Original book chapter ................................................................................................................... 13

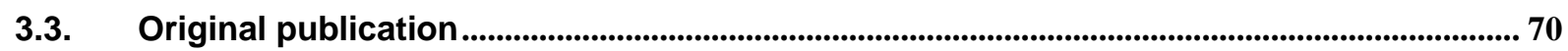

4. EFFECT OF EPO ON HIGHER BRAIN FUNCTIONS IN MICE .........................82

4.1. Overview of project II ...................................................................................................................... 82

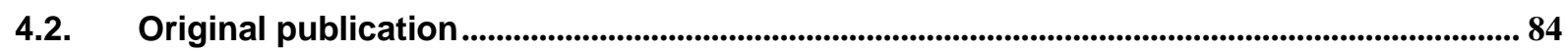

5. EFFECT OF OVER-EXPRESSION OF EPOR IN THE FOREBRAIN AND HIPPOCAMPUS OF MICE ON COGNITIVE PERFORMANCE ............................. 93

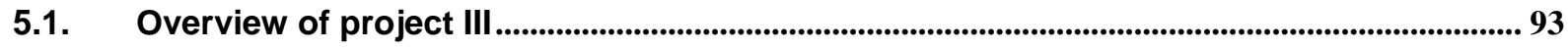

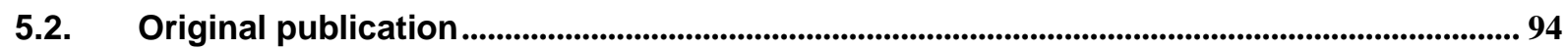

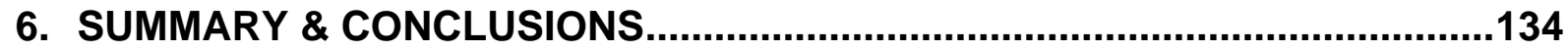

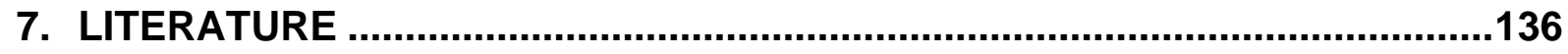

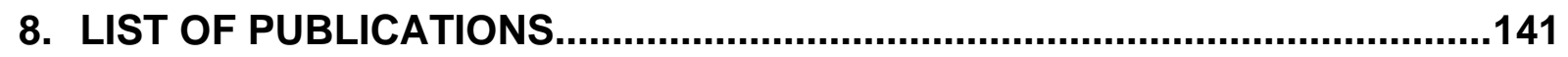

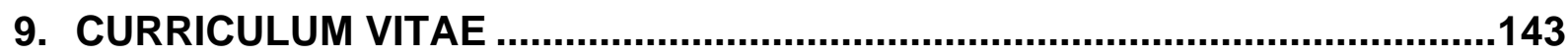




\section{INTRODUCTION TO THE SCIENTIFIC WORK}

Cognitive deficits and impairments of higher brain functions are the most burdening features in various neuropsychiatric disorders (e.g. Elvevag and Goldberg, 2000). Up to now, there are no satisfying proven treatments targeting the main cognitive domains affected in neuropsychiatric diseases (e.g. Stroup et al., 2006). In light of an increasingly aging society in industrialized countries and the herewith associated cognitive decline, novel neuroprotective / neuroregenerative approaches are desperately needed. The present cumulative thesis was conducted in the framework of the Göttingen Research Association for Schizophrenia (GRAS) and addresses two related topics: first, a novel experimental approach in mice combining genetic and environmental factors for modelling cognitive deficits relevant to schizophrenia, and second, the effect of erythropoietin (EPO) on improving higher cognitive functions (i.e. learning and memory, attention, executive functions) in mice. The thesis includes one book chapter and three original first-author publications. The chapter (El-Kordi, Radyushkin, Adamcio et al., 2009) gives a state-of-the-art overview of previous, present and future rodent models of schizophrenia. It highlights the urging need for valid and reliable animal models of this burdening disease. Moreover, it defines for the first time "DSM criteria for rodent schizophrenia". On these grounds, the first paper evolves (Radyushkin, El-Kordi et al., 2010) which combines a complexin2 null mutation in mice as a genetic factor and a mild parietal neurotrauma applied during puberty, as an environmental factor. This genetic-environmental model yielded several phenotypes relevant to schizophrenia. The second publication (El-Kordi, Radyushkin and Ehrenreich, 2009) demonstrates the effect of EPO treatment on several types of learning, attention and executive control in mice. It sheds light on methodological approaches to study these functions in rodents and uncovers the role of EPO in improving these domains in healthy mice. From there, I wanted to obtain more mechanistic insight into EPO mediated cognitive improvement. For that purpose, I characterized the cognitive performance of constitutively active EPOR overexpressing mice (cEPOR under the $\alpha$-calcium/calmodulin-dependent protein kinase II ( $\alpha$-CaMKII) promoter). This third paper shows the effects of EPOR on cognition when overexpressed in selected hippocampal and cortical neurons, which are highly relevant to higher brain functions (Sargin, El-Kordi et al., 2010, submitted). 
Impairments of higher brain functions are believed to be the core of various neuropsychiatric diseases, in particular schizophrenia (Elvevag and Goldberg, 2000). Schizophrenia is a debilitating disorder that affects approximately $1 \%$ of the population across cultures. It comprises positive symptoms (delusions, hallucinations), negative symptoms (social withdrawal, alogia, affective flattening) and cognitive symptoms (deficits in working memory, sustained attention and executive functions) (Wong and Van Tol, 2003). Knowledge about its neurobiological causes is still nascent. Accordingly, animal models of schizophrenia often lack construct validity. The observation that concordance rate in monozygotic twins amounts to only $50 \%$ made clear that environmental risk factors (e.g. neurotrauma, drug abuse, obstetric complications, etc.) likely act as necessary 'second hit' to trigger the disease process in a genetically predisposed individual. Previously, animal models with high face validity addressed only one factor, e.g. alterations in neurotransmitter systems, neonatal brain lesions, etc. (for review, see Lipska, 2004), and thus lack construct validity. Based on this 'second hit hypothesis' (e.g. Bayer et al., 1999; Maynard et al., 2001), recent animal models emerged unifying genetic and environmental factors (for review, see Ayhan et al., 2009).

Despite moderate success in mimicking schizophrenia in rodents, and recent pharmacological innovations in treatment, improving cognitive deficits remains a challenging task. Up to now, treatment of schizophrenia mainly addresses positive symptoms and is still based on antagonising the dopaminergic system, specifically blockade of D2 receptor (Miyamoto et al., 2005). New antipsychotic medications, so called atypical antipsychotics, act on partial blockade of several members of the serotonin receptor family (e.g. $5-\mathrm{HT}_{1 \mathrm{~A}}, 5-\mathrm{HT}_{2 \mathrm{~A}}, 5-\mathrm{HT}_{2} \mathrm{C}$ ) besides their affinity to $\mathrm{D} 2$ and other receptors (Mortimer, 2004). This new generation of antipsychotics have fewer undesirable side effects, in contrast to the old generation. However, they often lead to weight gain, sedation and metabolic alterations (for review, see Simon et al., 2009). Most importantly, in spite of attenuating positive symptoms and alleviating mood and motivation, they still have limited effects on improving cognition (for review, see Galletly et al., 2000). Nevertheless, in recent years, several reports appeared targeting neural systems implicated in the cognitive deficits observed in schizophrenia. Specifically $\alpha 7$-nicotinic agents proved to be potential promising 
candidates for ameliorating cognitive impairment (for review, see Buchanan et al., 2007; Radek et al., 2010).

Although often debated (Rund, 2009), limited neurodegenerative processes are almost always evident in the schizophrenic brain (Lieberman, 1999). Treating cognitive impairments in schizophrenia is so tedious due to the structural abnormalities, which seem to be pharmacologically non-modifiable (Harvey, 2009). The ongoing neurodegeneration in schizophrenics renders neuroregenerative / neuroprotective agents potentially attractive candidates for treating cognitive deficits in this disease. Neuroprotective compounds give a long-lasting improvement of symptoms and aim at slowing a continuous disease process (Sargin, Friedrichs et al., 2010 , in press). Interestingly, a recent study of our group demonstrated the effect of EPO on delaying gray matter loss in association with cognitive improvement in chronic schizophrenia (Wüstenberg et al., 2010).

Justifiably, in the last decade, EPO has in an unprecedented manner attracted attention as a candidate for neuroprotection / neuroregeneration (Sargin, Friedrichs et al., 2010, in press). Since its introduction to the clinic to treat chronic renal failure, several reports have been accumulating highlighting the effect of EPO on improving cognitive functions (Ehrenreich et al., 2008; Jelkmann, 1992). For a long time, the effect of EPO on cognition was attributed to its direct action on the hematopoietic system and to enhanced tissue oxygenation. However, several lines of evidence convincingly prove that EPO-mediated cognitive improvement is independent of its hematopoietic properties and represents a direct action in the brain (Ehrenreich et al., 2008; Sakanaka et al., 1998; Sirén et al., 2009; Zhang et al., 2009).

EPO is a 165 amino acid glycoprotein with a molecular mass of approximately 30.4 $\mathrm{kDa}$. It acts via binding to its specific transmembrane receptor, (EPOR), belonging to the cytokine type 1 receptor subfamily. Homodimerization of 2 transmembrane EPOR molecules leads to binding of a single EPO molecule and induces a conformational change which initiates EPOR-associated JAK2 transphosphorylation. Downstream signaling molecules are subsequently activated including signal transducers and activators of transcription (STATs), phosphatidylinositol-3 kinase (PI3K)/AKT, RAS/extracellular signal-regulated kinase (ERK1/2), nuclear factor kappa B (NF- 
kappa B) and calcium (Brines and Cerami, 2005; Sirén et al., 2001). Activation of these signaling cascades leads to further activation of anti-apoptotic factors, stimulation of cell differentiation, including induction of cellular shape-change and growth, or modulation of plasticity (Sargin, Friedrichs et al., 2010, in press).

The discovery of EPO/EPOR system in the brain (Masuda et al., 1993) made it a very promising candidate for treating brain disorders. In recent years, it has been shown that EPO is a potent agent for treating cognitive dysfunctions in several neuropsychiatric and neurological diseases (Ehrenreich, Fischer et al., 2007; Ehrenreich, Hinze-Selch et al., 2007). However, the mechanisms of its action on improving higher cognitive functions (e.g. attention and behavioral control) have not been studied so far.

\section{FOCUS OF MY PHD THESIS}

The present thesis addresses (1) a novel experimental approach in mice combining genetic and environmental factors for modelling cognitive deficits relevant to schizophrenia. (2) The effect of EPO on improving higher cognitive functions (i.e. learning and memory, attention, executive functions) in mice and the underlying physiological mechanisms. It includes 4 publications (one first-author book chapter, two first-author published papers and one first-author submitted paper).

\subsection{AIMS OF PROJECT I}

The first project addressed a novel experimental approach in mice, based on the 'second hit hypothesis' of schizophrenia. In this framework, a book chapter giving a state-of-the-art of past, present and future rodent models of schizophrenia has been published. The original study has been designed to investigate the effect of combining a complexin2 null mutation in mice (genetic predisposition) with a juvenile parietal neurotrauma as a second hit (environmental trigger). We hypothesized that only mutant lesioned mice will exhibit schizophrenia relevant behavioral, cognitive and MRI alterations. 


\subsection{AIMS OF PROJECT II}

The second project aimed at studying the effect of EPO on higher brain functions in mice. For that purpose we investigated under healthy conditions in absence of intervening disease variables the effect of chronic EPO administration on different types of learning and memory, attention and executive functions in 28-days old male C57BI/6 mice. In this study, we made use of a sequential operant paradigm, the five choice serial reaction time task (5CSRTT).

\subsection{AIMS OF PROJECT III}

To obtain a mechanistic insight in EPO-mediated cognitive improvement, we have designed the third study, investigating the effect of over-expression of EPOR in selected cell populations. For this purpose, we studied the cognitive performance of constitutively active EPOR overexpressing mice (CEPOR under the $\alpha$ calcium/calmodulin-dependent protein kinase II ( $\alpha$-CaMKII) promoter). These mice over-express the EPO receptor only in the pyramidal neurons of cortex and hippocampus. Their behavior was extensively analyzed using a wide behavioral test battery. 


\section{AN EXPERIMENTAL APPROACH COMBINING GENETIC AND ENVIRONMENTAL FACTORS FOR INDUCING A SCHIZOPHRENIA- LIKE PHENOTYPE IN MICE}

\subsection{OVERVIEW OF PROJECT I}

Schizophrenia is a frequent and devastating disease characterized by positive, negative, and cognitive symptoms (American-Psychiatric-Association, 2000; van Os and Kapur, 2009; Wong and Van Tol, 2003). Its underlying etiology is still poorly understood (Sawa and Snyder, 2002). Consequently, animal models addressing central constructs of the disorder are widely lacking. In contrast, models with face validity, where juvenile rodents are exposed to lesions or pharmacological interventions, have been used for many years (for review, see Lipska, 2004).

The genetic basis of this disorder is indisputable. However, the concordance rate in monozygotic twins is only $50 \%$ (Gottesman and Shields, 1976), pointing to additional, environmental risk factors (Bayer et al., 1999). Animal models reliably representing the disease phenotype should account for the interaction between genetic and environmental triggering factors. Based on the 'second hit hypothesis', we designed an experimental approach for modeling schizophrenia which combines a genetic (complexin2 ( $\mathrm{Cp} / \mathrm{x} 2$ ) null mutation) and an environmental factor (mild parietal cortical lesion during puberty) to induce schizophrenia-like phenotype in mice.

Since schizophrenia is seen as a "disease of the synapse" (Harrison and Weinberger, 2005) synaptic molecules moved into research focus. Among others, complexins (CPLX), which are presynaptic proteins that modulate neuronal exocytosis (for review, see Brose, 2008), have been associated with schizophrenia (Harrison and Eastwood, 1998; Lee et al., 2005; Sawada et al., 2002). Previous studies characterizing $\mathrm{Cp} / \times 2^{-/}$mice reported a mild behavioral phenotype consisting of slight motor deficit and impaired learning in Morris water maze (Glynn et al., 2003; Glynn et al., 2007; Yamauchi et al., 2005). Regarding environmental factors, neurotrauma (Malaspina et al., 2001; McAllister, 1998), and particularly lesions affecting the right parietal lobe have been linked with schizophrenia-like psychosis (Sachdev et al., 2001; Zhang and Sachdev, 2003). Several years ago, we have developed a mouse model, based on findings of a longitudinal study on patients with childhood onset schizophrenia (Thompson et al., 2001). In our mouse model (Sirén et 
al., 2006) a small standardized lesion is set during puberty onto the right parietal cortex which initiates a bilateral neurodegenerative process very similar to that occurring endogenously in schizophrenia for - up to now - unknown reasons (Sargin et al., 2009).

By combining $C p / \times 2$ null mutation with neurotrauma, we provide further experimental evidence that a genetic predisposition (affecting neurodevelopment) in addition to an environmental risk factor (triggering neurodegeneration) can give rise to alterations relevant to schizophrenia in mouse behavior and brain morphology.

Several months after lesion we found, that only $C p / \times 2$ null mutants showed reduced pre-pulse inhibition, deficit of spatial learning, and loss of inhibition after MK-801 treatment. Forced alternation in T-maze, object recognition, social interaction and elevated plus maze tests were unaltered in all groups. The previously reported mild motor phenotype of Cplx2 null mutants was accentuated upon lesion. MRI volumetrical analysis revealed a decrease of hippocampal volume exclusively in lesioned Cplx2 null mutants. 


\subsection{ORIGINAL BOOK CHAPTER}

El-Kordi A, Radyushkin K, Adamcio B and Ehrenreich H (2009). Rodent models of schizophrenia: Past, present and future. In: Endophenotypes of Psychiatric and Neurodegenerative Disorders in Rodent Models. Ed. Granon S. Signpost.

Personal contribution

I was responsible for literature search and writing the chapter. 
Transworld Research Network

37/661 (2), Fort P.O., Trivandrum-695 023, Kerala, India

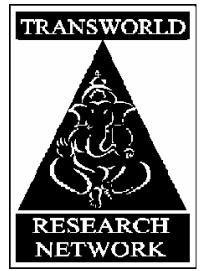

Endophenotypes of Psychiatric and Neurodegenerative Disorders in Rodent Models, 2009: 209-264 ISBN: 978-81-7895-402-8 Editor: Sylvie Granon

\title{
Rodent models of
} schizophrenia: Past, present and future

\author{
Ahmed El-Kordi, Konstantin Radyushkin, Bartosz Adamcio \\ and Hannelore Ehrenreich \\ Division of Clinical Neuroscience, Max-Planck-Institute of Experimental \\ Medicine, Göttingen, Germany
}

\begin{abstract}
Schizophrenia is a devastating disease that affects approximately $1 \%$ of the population all over the world and across cultures. Up to now, neither satisfying treatment nor fundamental understanding of etiology and pathogenesis of this heterogeneous group of diseases are available. In contrast to many other brain diseases, schizophrenia has long been seen as a mysterious, non-organic and stigmatising condition. Even nowadays, the point of view exists
\end{abstract}

Correspondence/Reprint request: Dr. Hannelore Ehrenreich, Division of Clinical Neuroscience, Max-PlanckInstitute of Experimental Medicine, Göttingen, Germany. E-mail: Ehrenreich@em.mpg.de 
that schizophrenia cannot affect species other than humans and requires developed language to manifest itself. The first animal models of schizophrenia-associated symptoms evolved when antipsychotic drugs had to be tested for efficacy in preclinical studies. These first models were crude, aimed at creating a phenotype that could be influenced by antipsychotics but did not model any real features of schizophrenia. In this regard, major advance in the field came within the last years. Animal models have been developed that try to cover various aspects of schizophrenia, i.e. positive symptoms (particularly hyperactivity), negative symptoms (social behavior and withdrawal) and cognitive dysfunction (working memory, attention). There is not only improvement in defining suitable animal models, but also in the validity of behavioral paradigms used for classifying schizophrenia-like phenotypes in rodents. This chapter will summarize "DSM criteria for rodent schizophrenia", describe (1) models that have been used in the past, (2) models that have been introduced recently and (3) models that are being developed, combining genetic and environmental factors, in order to more adequately represent the disease phenotype. To conclude, an animal model of schizophrenia is desperately needed not only to improve prevention and treatment options for schizophrenic patients, but also to gain insight into relevant pathophysiological processes leading to the disease.

\section{Abbreviations}

\begin{tabular}{|c|c|c|}
\hline AP5 & : & dl-2-amino-5-phosphonovaleric acid \\
\hline ARVCF & 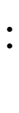 & $\begin{array}{l}\text { Armadillo repeat deleted in velocardiofacial } \\
\text { syndrome }\end{array}$ \\
\hline BDNF & : & Brain-derived neurotrophic factor \\
\hline сAMP & : & cyclic adenosine monophosphate \\
\hline COMT & : & Catechol-O-methyl transferase \\
\hline COS & : & Childhood-onset schizophrenia \\
\hline CPLX2 & : & Complexin 2 \\
\hline $\mathrm{DH}$ & : & Dorsal hippocampus \\
\hline DISC1 & : & Disrupted-in-schizophrenia 1 \\
\hline DLPFC & : & Dorsolateral prefrontal cortex \\
\hline DSMIV & : & $\begin{array}{l}\text { Diagnostic and Statistical Manual of Mental } \\
\text { Disorders, 4th ed. }\end{array}$ \\
\hline DTNBP1 & : & Dystrobrevin-binding protein 1 \\
\hline FEZ1 & : & Fasciculation and elongation zeta- 1 protein \\
\hline fMRI & : & functional magnetic resonance imaging \\
\hline GABA & & Gamma aminobutyric acid \\
\hline GAD-67 & & Glutamate decarboxylase 67 \\
\hline
\end{tabular}




\begin{tabular}{lll}
\hline HPS7 & $:$ & Hermansky-Pudlak syndrome type 7 \\
KO & $:$ & Knock-out \\
LI & $:$ & Latent inhibition \\
LIS1 & $:$ & Lissencephaly 1 protein \\
LTP & $:$ & Long term potentiation \\
MPFC & $:$ & Medial prefrontal cortex \\
NDEL1 & $:$ & Nuclear distribution element-like 1 protein \\
NMDA & $:$ & N-methyl-D-aspartate \\
NRG1 & $:$ & Neuregulin 1 \\
PCP & $:$ & Phencyclidine \\
PD & $:$ & Postnatal day \\
PDEB4 & $:$ & Phosphodiesterase 4B \\
PFC & $:$ & Prefrontal cortex \\
PPI & $:$ & Prepulse inhibition \\
RGS4 & $:$ & Regulator of G-protein signaling 4 \\
SNP & $:$ & Single nucleotide polymorphism \\
STG & $:$ & Superior temporal gyrus \\
THC & $:$ & Tetrahydrocannabinol \\
TTX & $:$ & Tetrodotoxin \\
VGluT-1 & $:$ & Vesicular glutamate transporter 1 \\
VH & $:$ & Ventral hippocampus \\
WCST & $:$ & Wisconsin Card Sorting Test \\
5CSRTT & $:$ & Five Choice Serial Reaction Time Task
\end{tabular}

\section{Introduction}

Schizophrenia is a devastating brain disorder characterized by positive symptoms (e.g. delusions, hallucinations and formal thought disturbances), negative symptoms (e.g. social withdrawal, affective flattening and alogia) and cognitive deficits (e.g. attention, executive functions and working memory) [1]. The latter are getting more and more in the focus of research and are thought to be central to the social and behavioral disability of schizophrenic patients [2, 3]. According to the Diagnostic and Statistical Manual of Mental Disorders (4th edition; DSM-IV) of the American Psychiatric Association [4], the estimated lifetime prevalence of schizophrenia is between $0.5 \%$ and $1 \%$. This estimation is found across all cultures worldwide and has remained unchanged since 1900 [5]. The heterogeneity of symptoms of schizophrenia as well as the lack of clearly measurable disease specific pathology [6], together with the fact that manifestation of this complex disorder in humans is most reliably diagnosed through language make developing rodent model of schizophrenia a difficult and challenging undertaking. 
This chapter takes the reader to a time travel through the history of rodent models of schizophrenia, without intending to review all possible animal models of schizophrenia and their theoretical underpinnings. It is the attempt to illustrate the different stages and conceptual frameworks of the development of valid and reliable rodent models of schizophrenia. For primate models, the interested reader is referred to the review of Ellenbroek and Cools [7]. The difficulty of reliably modeling mental disorders, believed to be quite uniquely "human", in simpler model systems such as mice, makes the formulation and fulfilling of validity criteria an imperative condition. The next section discusses criteria which have to be met in an animal model of mental disorders in general and of schizophrenia in particular. An overview is provided about schizophrenia symptoms which have a clear homology in rodents and thus are translatable to a simpler organism.

\section{Validity issues in animal models of psychiatric disorders}

Animal models for psychiatric disorders are becoming an indispensable tool in investigating the mechanisms that underlie a human disease. Moreover, they are of great importance for the development of new treatment strategies. This is not only the case for animal models for physical disorders, such as diabetes mellitus and hypertension, it also holds true for mental disorders like depression and schizophrenia. But modeling mental disorders in rodents is more complex and challenging compared to modeling a physical condition with clear-cut pathophysiological mechanisms and well-defined diagnostic criteria. As Kornetsky and Markowitz [8] reported, animal models of physical disorders have the attribute of close similarity between the physiologic condition in animals and humans. But even in these areas, similar behaviors in various species may exist for different reasons, and therefore, have different meanings [9]. Nevertheless, modeling a highly cognitive condition in a cognitively lower organism has to be regarded as an experimental compromise. As McKinney and Moran [9] pointed out, an animal model can never be exactly the same as the condition being modeled. Accordingly, prevailing animal models do not attempt to represent a complete animal equivalent of the human condition. This is obvious when modeling such heterogeneous brain disorder as schizophrenia. Rather, animal models are developed to study specific causative or mechanistic aspects of the corresponding disease [10].

There are different levels on which an animal model may represent a disease: 1. It may reproduce inducing factor(s); 2. It may mimic phenomenology and 3. It may show responsiveness to already existing treatments [11]. Thus, the degree 
to which an animal model accurately mimics the real human condition varies according to the aspects that it intends to represent [11]. In this context, the concept of validity is of great importance. Initially, McKinney and Bunney [12] proposed minimal requirements an animal model has to meet: 1 . similarity of inducing conditions; 2. similarity of behavioral states produced; 3. common underlying biological mechanisms; and 4 . reversal by clinically effective treatment techniques [adapted from ref. 9, see also ref. 12]. Later, Willner [13] pointed out, that animal models should possess predictive, face and construct validity. Although this was originally stated in the framework of animal models for depression, it also holds true for other animal models of psychiatric disorders, including schizophrenia.

Predictive validity, in its broadest sense, concerns the ability of a model to make successful predictions or to forecast, for example, the behavior of the animal in a specific experimental situation. A model with high predictive validity allows to draw extrapolation of the effect of a given experimental manipulation from one species (e.g. rodents) to other species (e.g. humans), and from one setting (e.g. laboratory) to another one (e.g. "real life") [14]. In a more narrow sense, predictive validity implies that treatments proven to be effective in humans have also to be effective in the animal model. This notion is quite similar to the concept of pharmacological isomorphism proposed by Matthysse [15]. For the purpose of this chapter, we use the term predictive validity in its narrow sense.

The second validity issue is that of face validity. Face validity implies, in its simplest definition, the similarity of symptomatology. This concept is similar to the criterion of behavioral isomorphism by Kornetsky and Markowitz [16] and the concept of symptom similarity by Matthysse [15]. Symptoms elicited in animal models with high face validity resemble those seen in humans.

The third aspect of validity which animal models have to fulfill is the concept of construct validity. It is believed to be the most important aspect in validating animal models of complex disorders such as schizophrenia [14]. This concept refers to the theoretical background or constructs underlying the disease process. Animal models with construct validity have a sound theoretical rationale. Thus they go in accordance with constructs characterizing schizophrenia patients. For example, animal models are based on the construct that schizophrenics have a deficient information processing.

According to Ellenbroek and Cools [7], predictive validity has the lowest, while construct validity has the highest hierarchy of validity issues. Concerning animal models of schizophrenia in particular, various researchers proposed specific and theoretically sound criteria, which have to be met in an animal model trying to mimic the disease's pathophysiology, 
symptomatology, etiology, and to pave the way for developing new treatment strategies. For example, Matthysse and Haber [17] defined standards which a model of schizophrenia has to satisfy. Although most of these standards were drug related, and thus fit perfectly well to a pharmacological animal model of schizophrenia, these criteria may be incorporated with other complementing standards addressing other issues, such as etiology and cognitive symptomatology. For instance, Ellenbroek and Cools [7], proposed criteria adapted from Willner [18] addressing aspects of predictive, face and construct validity. Reviewing these standards with their corresponding empirical background is beyond the scope of this chapter. The interested reader is referred to $[7,18]$.

After discussing standards guiding the development and assessment of animal models for psychiatric disorders, with a main focus on schizophrenia, how should a rodent model mimicking the schizophrenia symptomatology and possessing predictive, face and construct validity look like? Which symptoms do rodents show that may be translatable to disturbances seen in humans with schizophrenia? And how can these symptoms and behavioral traits be reliably measured? As mentioned earlier in this chapter, modeling schizophrenia is a great challenge for behavioral neuroscientists, not only due to the heterogeneity of symptoms, but also due to the simple fact, that rodents do not possess a crucial tool, which has a diagnostically indispensable value, that is verbal communication. As McKinney and Moran [9] stated, no animal analogies exist for the characteristics of auditory hallucinations, flatness of affect, thought alienation, thoughts spoken aloud, delusions of control, voices speaking to the patient to just name a few [see also ref. 9]. However, rodents have a wide behavioral repertoire, and their lack of human-like verbal communication should not discourage researchers from trying to model these symptoms.

The next section will focus on behavioral domains an animal model of schizophrenia has to show to be regarded as valid. We will illustrate the symptoms in schizophrenia patients with their corresponding behavioral traits in rodents and behavioral paradigms used to assess these traits.

\section{Behavioral domains affected in schizophrenia}

In the context of modeling brain disorders in rodents, many people wonder: “How does a schizophrenic mouse look like?” In our opinion, and based on human phenomenological data, a rodent model for schizophrenia should not show complete cognitive disability. Despite the fact that schizophrenia is a burdening and devastating brain disorder, which compromises normal social interaction, patients do not have a general intellectual disability comparable to that seen in patients suffering from mental retardation. Thus, what has to be 
modeled is not debility or apparently eccentric and bizarre behavior in each and every respect. Rather, subtle deficits in particular cognitive domains, ideally under specific conditions and experimental manipulations, should be present in a valid model of schizophrenia. In the last years, many researchers made very accurate attempts to relate rodent behaviors of potential relevance to clinical phenomena in schizophrenia $[11,19]$. Table 1 summarizes the main domains or symptom clusters found in schizophrenia with their corresponding findings in humans and the behavioral aspects, which have to be proven in a rodent model of schizophrenia. Figure 1 illustrates some of the behavioral set-ups for testing and assessing positive (A) and negative symptoms (B) as well as cognitive functions (C, D) mentioned in Table 1. The following section will illustrate behavioral and cognitive domains implicated in schizophrenia, amenable to modeling in rodents.

Table 1. Schizophrenia symptoms which might be mimicked in a rodent model.

\begin{tabular}{|c|c|c|}
\hline Domain & Schizophrenia in humans & Rodent phenotype \\
\hline \multirow[t]{3}{*}{$\begin{array}{l}\text { Positive } \\
\text { symptoms }\end{array}$} & $\begin{array}{l}\text { Hallucinations, delusions, thought } \\
\text { disorders }\end{array}$ & Lack of reliable readouts in rodents \\
\hline & Psychomotor agitation & $\begin{array}{l}\text { Spontaneous hyperlocomotion } \\
\text { Increase in PCP- or MK-801-induced } \\
\text { hyperlocomotion } \\
\text { Increase in amphetamine-induced } \\
\text { hyperlocomotion } \\
\text { Increase in stress-induced hyperlocomotion } \\
\text { Reduced haloperidol-induced catalepsy }\end{array}$ \\
\hline & Stereotypy & Circling movements, repetitive behaviors \\
\hline \multirow[t]{3}{*}{$\begin{array}{l}\text { Negative } \\
\text { symptoms }\end{array}$} & Social withdrawal & $\begin{array}{l}\text { Decreased preference to social novelty } \\
\text { Decreased social interaction } \\
\text { Decreased nesting in the home cage } \\
\text { Decreased huddling in the home cage }\end{array}$ \\
\hline & Affect flattening & Lack of reliable readouts in rodents \\
\hline & Anhedonia & Decrease in sucrose preference \\
\hline \multirow[t]{4}{*}{$\begin{array}{l}\text { Cognitive } \\
\text { symptoms }\end{array}$} & Working memory deficits & $\begin{array}{l}\text { Impaired performance in T-maze } \\
\text { Impaired performance in 8-arm maze }\end{array}$ \\
\hline & Deficit of attention & $\begin{array}{l}\text { Impaired latent inhibition } \\
\text { Decreased sustained attention in 5CSRTT } \\
\text { Increased distractibility in 5CSRTT }\end{array}$ \\
\hline & Executive function deficits & $\begin{array}{l}\text { Increased reaction time in 5CSRTT } \\
\text { Increased premature responses in 5CSRTT } \\
\text { Decreased set-shifting ability }\end{array}$ \\
\hline & Disturbance of sensorimotor gating & Diminished PPI, retarded startle habituation \\
\hline
\end{tabular}



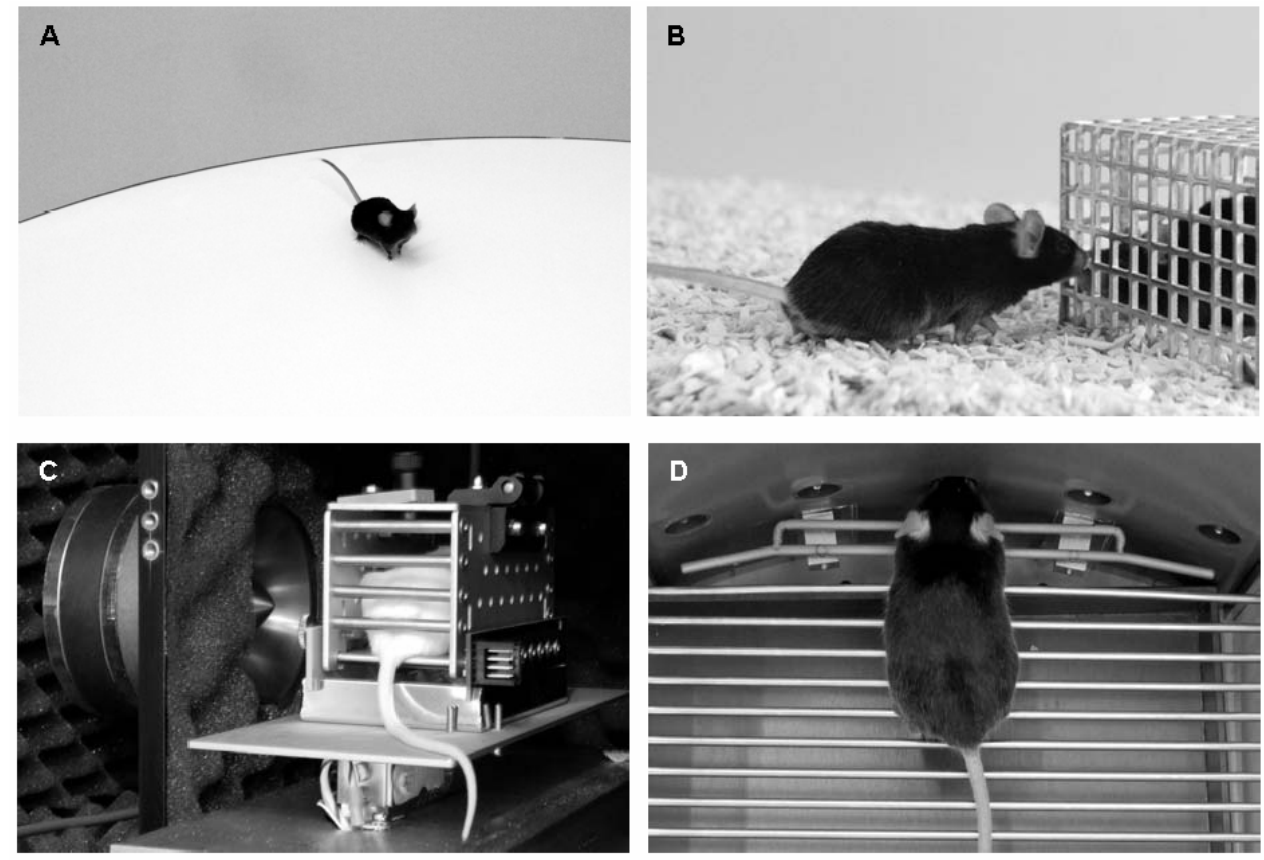

Figure 1. Experimental paradigms to address schizophrenia-like behavioral traits in mice. A. Hyperactivity in open field as evidence for psychomotor agitation. The behavior of a mouse, placed in a big circular or rectangle arena, is recorded by a computer, equipped with a video-tracking system. Total distance traveled and pike velocity serve as readouts of motor hyperactivity, which in turn are seen as indirect evidence for positive symptoms of schizophrenia. B. Tri-partite test for sociability and social memory in mice. In an experimental chamber with 3 compartments, the mouse has the option to stay (1) in close proximity with an unfamiliar mouse, kept in a small wire cage, (2) in the compartment with an empty cage or (3) in the central compartment. The proportion of time spent in interaction with the unfamiliar mouse is used as a readout for sociability. In order to evaluate social memory, the test mouse, right after sociability testing, is offered the choice between two mice - one that is familiar and one that is new. The proportion of time spent in interaction with the new mouse (normally higher as compared to the familiar mouse) is the readout for social memory. C. Prepulse inhibition of the startle response. Like many species, including Homo Sapiens, mice show a startle response to a sudden acoustic stimulus. To measure the amplitude of such a response, the mouse is placed in a small restriction cage on a platform, attached to a vibration sensor. The amplitude of oscillations, evoked by body muscle contractions, triggered by a $120 \mathrm{~dB}$ white noise pulse, is recorded by a computer. The mouse responses are measured in two types of trials - with and without a weak stimulus (prepulse), applied 50-500msec before the pulse. The degree of response reduction in "prepulse trials" in comparison to "pulse only trials" is calculated. D. Five choice serial reaction time task as a test of attention. In order to get a reward, the mouse has to make a nosepoke response into one of 5 holes where a light signal is randomly presented for a short period of time $(0.8 \mathrm{sec})$. The number of omitted signal presentations serves as a main readout for attention. In order to further characterize the attention of the mouse, additional trials with application of distracting stimuli are used. 


\subsection{Positive symptoms}

As mentioned earlier in this chapter, there is no assessable rodent behavior equivalent to hallucinations and disorganized thoughts [9]. Thus, investigators have to rely on other overt behavioral traits indicative of positive symptoms. Interestingly, modeling positive symptoms in rodents, initially in rats, has a long tradition in the context of studying the effect of antipsychotic drugs. Hyperlocomotion is seen as a valid indicator for positive symptoms in rodents. Thus, several studies have used spontaneous hyperlocomotion and increased vertical (i.e. rearing) and horizontal motor activity in the open field as a measure for positive symptoms in rodents [20-24].

\subsection{Stereotypy}

Stereotypy is a repetitive, unvarying and seemingly functionless behavior found in various psychiatric disorders. Repetitions can be manifest at different functional levels. For example, in the motor domain (tics and tremor), executive motor functions (rituals), cognitive domain in form of preoccupation with specific thoughts (rumination) and in planning and strategic domain, resulting in cognitive inflexibility [25]. Stereotypy has often been regarded as a hampering symptom of schizophrenia. It comprises different behavioral features, e. g. brief motor tics, mannerisms and complex ritualistic behavior [25]. These disturbances seem to be due to excess of subcortical dopaminergic activity [26]. Another phenomenologically similar symptom, i.e. perseverations, has been also implicated in schizophrenia. Perseverations are also repetitive hampering behaviors. However, they are often described in the context of mental states. Ridley [25] defines stereotypy as the excessive production of one type of motor act or mental state, whereas perseveration refers to a restriction of choices of action while the repetitive behavior is not excessive. We will not discuss modeling perseverations in rodents, as there are barley any data pointing to this feature. Stereotypy has been documented in various species, especially in captive animals [e.g. ref. 27]. This phenomenon is widely spread among laboratory animals [for review, see ref. 28]. In rats, administration of dopamine agonists (e.g. apomorphine) has been reported to induce stereotyped locomotion, sniffing, licking and gnawing [29]. Thus, rodents bear the ability of showing stereotyped behavior, moreover, this also depends on genetic and environmental factors [29].

\subsection{Negative symptoms: Social behavior}

Social deficits are believed to be a hallmark of schizophrenia and belong to negative symptoms of the disease. Social functions including interacting and communicating with others, maintaining employment and having a functional 
role in community are affected in various psychiatric disorders, however, they are characteristic for schizophrenia [30]. Social deficits are even apparent long before the disease onset. Moreover, it is widely recognized that social and cognitive functions are interrelated [for review, see ref. 31]. Even though rodents manifest a limited spectrum of social behaviors, they enable measuring functions like social interest, interaction and social preference. Furthermore, rodents emit ultrasonic vocalizations in various contexts [32], another trait of social behavior and communication, recently exploited for the demonstration of autistic symptoms in a mouse model of monogenic heritable autism [33]. Social traits have been often assessed in rodent models of schizophrenia. Another feature of negative symptoms is flattened affect and anhedonia. Whereas flattened affect is virtually not assessable in rodents, measuring anhedonia and reward sensitivity has a long tradition. A classic method of assessing anhedonia in rodents is sucrose preference [for review, see ref. 34].

\subsection{Cognitive functions: Prepulse inhibition and latent inhibition}

Prepulse inhibition (PPI) of the acoustic startle response is a valuable and traditional measurement widely used in schizophrenia research. It has been repeatedly reported that schizophrenia patients manifest deficits in PPI [for review, see ref. 35]. PPI is a measure for sensorimotor gating and preattentive information processing [36]. Detailed description of this procedure as well as parametric issues have been reviewed elsewhere [37]. A wealth of studies have reported the effect of dopamimetics, NMDA antagonists and antipsychotics on PPI performance of rodents [38].

Another paradigm with great experimental tradition is latent inhibition (LI). Latent inhibition is defined as the poor evidence for learning a stimulus that previously was presented without any consequence, as compared to a novel or previously attended stimulus [39]. LI is believed to be a measure for attentional processes, which are severely disrupted in schizophrenia patients, although data of LI in some schizophrenia patients delivered also conflicting results [40]. Several dopaminergic agonists as well as glutamatergic compounds disrupt LI [41, 42]. It has also been demonstrated, that clozapine reverses the amphetamine-induced LI deficits in rats [43]. Various studies have used LI in the framework of establishing animal models of schizophrenia. Some of these will be discussed later in this chapter.

\subsection{Memory}

Perhaps one of the most intensively studied affected cognitive domains in schizophrenia is memory. In particular, working memory has gained a 
considerable amount of scientific attention. Deficits in memory processes have been consistently reported in individuals with schizophrenia [for review, see ref. 44]. Several studies have demonstrated altered activation of prefrontal cortical brain regions, substantially involved in executive functions and working memory [e.g. refs. 45, 46]. Notably, deficits in working memory are believed to be a behavioral and cognitive marker for schizophrenia, as some studies have demonstrated that working memory deficits are specific to schizophrenia, not to other mental disorders [47]. Working memory is, simply speaking, the ability to hold information in temporary storage to successfully complete the running task [48]. Again, rodents have a wide repertoire of behavioral traits, thus allowing assessment of memory functions. Due to space limitations, we will not review tasks used to assess memory in rodents. Several papers give an extensive and adequate overview of memory tasks, predominantly working memory, of rodents [49, 50].

Taken together, rodents possess various behavioral traits which are analogous to clinical domains implicated in schizophrenia. This provides the ground for developing rodent models of the disease. In the following sections, existing rodent models will be discussed and evaluated on the basis of their validity issues.

\section{3. "Past"}

In this part we will discuss animal models which have been used in the past. The time categorization "past, present and future" should not be understood as a judgment of the scientific impact of these models. Rather, we are using this classification to illustrate the scientific trend and to describe the progress, based on clinical and basic research, made in the context of modeling schizophrenia in animals. For the purpose of this chapter, we adapt the definition of animal models made by Marcotte and colleagues [10]. Thus, an animal model is "an experimental manipulation that elicits behavioral and neurochemical changes that can be related to schizophrenia using the criteria for predictive, construct and face validity" [10]. Reliable and well characterized indicators of schizophrenia in humans such as deficient information processing and pre-attentive functions as measured by prepulse inhibition [51] and latent inhibition should not be considered animal models for schizophrenia in their own right. These are valid behavioral paradigms widely used in schizophrenia research and are therefore important single tools within a battery characterizing the behavioral phenotype of a potential animal model.

For simplicity, "past" animal models are here subdivided into two big groups: 1. Pharmacological models and 2. Neurodevelopmental models. 


\subsection{Pharmacological animal models}

Perhaps the most famous hypothesis on the pathophysiology of schizophrenia is the dopamine hypothesis of schizophrenia [52, 53]. In brief, the hypothesis states, that there is an excess of dopamine in the brains of schizophrenia patients [53]. This hypothesis was expanded and revised in the last years, also to account for negative and cognitive symptoms of schizophrenia [for review, see refs. 54, 55]. Nowadays, it is widely believed, that some deficits seen in schizophrenia are due to two dopamine related processes: Hyperactive dopamine system in mesocortical brain regions, accounting for positive symptoms, and hypoactive prefrontal dopamine system resulting in negative symptoms and cognitive deficits [55, 56].

Effects of amphetamine administration on non-schizophrenics [57] as well as reports describing schizophrenia-like symptoms elicited by amphetamine (so called "amphetamine psychosis", [58]) were some hints directing to the formulation of the dopamine hypothesis of schizophrenia. It is thus not surprising that the first rodent models of schizophrenia were those using dopamimetics to elicit schizophrenia-like symptoms. Administration of amphetamine and related psychotomimetics in rodents reliably elicits hyperlocomotion and stereotypy [23], behavioral alterations that are widely believed to be schizophrenia relevant traits analogous to positive symptoms in humans [19]. As mentioned earlier in this chapter, the correspondence between rodent "positive symptoms" and positive symptoms in schizophrenia patients might be debatable. However, the reliable replications of this finding in rodents, as well as the involvement of the relevant neurotransmitter systems make this feature of dopamimetics administration very useful for comparison among species and different models [10].

Animal models using psychotomimetics have also another important feature: Dopamine agonists disrupt PPI. This is the case for humans [59] as well as for rodents [60]. Based on the finding, that schizophrenia patients have disrupted PPI [61, 62] and that disruptions in sensorimotor gating and information processing are believed to be some of the characteristics of the disorder, pharmacological rodent models of schizophrenia, at least those using dopamimetics, seem to have at least some construct validity. Interestingly, antipsychotic medication, such as clozapine, reverses PPI deficits in some schizophrenia patients [63] and in apomorphine-treated rodents [64]. To sum up, previous rodent models using psychotomimetics or dopamine agonists succeeded in eliciting some core features of schizophrenia in rodents. Despite fairly good predictive and face validity, the construct validity is still limited [10], due to ignoring the genetic basis of the disease 
[e.g. ref. 65]. On the other hand, it is not surprising, that pharmacological models have good predictive validity as they elicit psychosis in healthy humans [57] and deteriorate symptoms in schizophrenia patients [66]. Nevertheless, the involvement of dopamine and its interactions with other neurotransmitter systems in the pathophysiology of schizophrenia is not yet resolved. Moreover, there are some treatment-resistant schizophrenia patients, who do not benefit from dopamine antagonists [67, 68]. Also the lack of responding of negative symptoms upon classic antipsychotic medication reduces the construct validity of dopamine models.

Not only dopamimetics attracted attention regarding their potential involvement in the pathophysiology of schizophrenia. The administration of the dissociative anesthetic phencyclidine (PCP) - and its analogue ketamine in healthy subjects was reported to produce a syndrome closely resembling schizophrenia with positive symptoms (paranoia, hyperlocomotion and auditory hallucinations), negative symptoms (social withdrawal, affective flattening and anhedonia) and cognitive symptoms (e.g. impaired working memory) [69]. PCP and ketamine are noncompetitive NMDA glutamate receptor antagonists. Their schizophrenia resembling effect was first discovered during their initial use as anesthetics [70]. Clinical data showed decreased glutamate levels in the cerebrospinal fluid of schizophrenic patients [71]. Moreover deficits in PPI due to administration of NMDA antagonists are reversed by clozapine, an atypical antipsychotic [72, 73]. Based on the effect of PCP in healthy volunteers, it was proposed that schizophrenia may involve reduced NMDA receptor function [74, 75]. In rodents, PCP and related substances such as MK-801 produce a picture similar to that in humans. Intrastriatal injections of a selective NMDA receptor antagonist, AP5, in rats led to a selective impairment in working memory (T-maze) performance, while spatial memory (radial arm maze) was intact [76]. Also in the rat, administration of MK-801 and PCP significantly disrupted PPI [77]. Moreover, rats treated with PCP had deficits in social behavior, while the typical antipsychotic haloperidol reversed some of these deficits [78]. For a review on rodent glutamate models of schizophrenia, the reader is referred to [74].

Although the glutamate hypothesis of schizophrenia may bear many similarities with the clinical manifestation of schizophrenia [for a discussion see ref. 74] and possesses good predictive and face validities, again, as the case with the dopamine hypothesis, the construct validity is limited [10]. For example, some findings in PPI are highly dose dependant. In some cases, sub-anesthetic PCP and ketamine administration did not have any effect on PPI. In other cases, it even elicited an enhanced inhibition [79]. Indeed, there are various interactions between the glutamatergic and dopaminergic systems 
[80] and both seem to be involved in the neurobiology of schizophrenia. But the way how one neurotransmitter system or another elicits/affects the wide spectrum of symptoms over the course of the disease remains unclear.

To conclude, pharmacological animal models addressing different neurotransmitter systems, involved in the pathophysiology of schizophrenia, are undoubtedly of great value for unraveling of disease and treatment mechanisms. Nevertheless, pharmacological models, directly manipulating specific neurotransmitter systems, could not account for some other features of schizophrenia, for example neuroanatomical abnormalities in patients [81] and relatives [82], or cognitive deficits in first-degree relatives $[83,84]$. These and other features are out of the scope of direct pharmacological induction. Other models were developed to address aspects of disease dynamics and course. These models will be discussed in the next section.

\subsection{Neurodevelopmental models}

\subsubsection{Clinical evidence and theoretical basis}

As was shown in the section before, most conventional animal models have studied effects of direct manipulations of specific neurotransmitter systems, especially dopamine. This is due to the strong implications of dopamine in the disorder. However, it has become clear that models based on direct manipulations of the dopaminergic system have a limited heuristic ("serving to discover") value [11]. Moreover, they do not address mechanisms involved in either cortical deficits [85] or the postpubertal onset, that also characterizes schizophrenia. Structural abnormalities in the brains of schizophrenia patients have been excessively documented and led to the assumption, that degenerative and/or neurodevelopmental processes are involved in the etiology of the disorder. For example, enlargement of the lateral and third ventricles [86], reduced volume of cortical grey matter [87] and smaller anterior hippocampi [86] were some widely reported findings. The reductions of grey matter do not seem to be a general deficit; rather it appears that they affect association cortices such as those located in the superior temporal gyrus, in the dorsal prefrontal cortex and in limbic regions including hippocampus and anterior cingulate [81, 88]. Importantly, many of these abnormalities were found in first-episode [89], never medicated subjects. Thus, the structural pathology seems to reflect a primary disease process [3].

The hippocampus and the prefrontal cortex (PFC) proved to be imperative for the pathology of the disease, both due to structural abnormalities [81, 88, 90, 91] and partially corresponding cognitive defects 
[92, 93], a core feature of the disease. For instance, reduced regional cerebral blood flow in the dorsolateral PFC (DLPFC) in chronic schizophrenia during performance of an established executive task, the WCST [85, 93] was reported. The discovery that the prefrontal cortex could regulate subcortical dopamine [94] offered a promising link between the suggested subcortical dopamine dysregulation and data assuming the involvement of the PFC in schizophrenia [93]. Other data also consistently reported cell loss in the hippocampus [95], while initial reports of hippocampal neuron misplacement in the superficial layers of the adjacent entorhinal cortex have not been replicated in various following studies [96]. However, PFC structural abnormalities were less consistently replicated, while hippocampal pathology was most reliably observed [for review, see ref. 81].

There has been a considerable debate, whether the observed structural abnormalities and clinical manifestations are due to neurodegenerative processes or whether they are a result of neurodevelopmental deficit [97, 98]. The clinical deterioration [99] as well as the progressive cortical grey matter loss [100] may reflect the involvement of degenerative processes. On the other hand, ventricular enlargement and size do not seem to correlate with the duration of untreated illness [101]. Whereas gliosis in postmortem studies [102], evidence for most degenerative processes [103], is lacking, recent evidence suggests a role of apoptotic mechanisms in the disease process [104, 105]. Interestingly, schizophrenia patients do not seem to carry an increased risk of Alzheimer disease [106]. It has also been reported, that intellectual and cognitive functions do not much further decline with age in schizophrenia [107]. Taking all these points into account, schizophrenia is a primarily neurodevelopmental disorder, with neurodegenerative processes contributing to pathophysiology and disease progression.

Neurodevelopmental models of schizophrenia test the hypothesis that schizophrenia is caused by a defect in brain development [108]. Some models attempt testing etiological theories, studying the role of maternal malnutrition [109, 110], viral infections during pregnancy [111] and obstetric complications [112]. Others address the impact of disrupted neuronal maturation and synaptogenesis [113] and the relevance of early stressful events [114]. Discussing all these models in detail is out of the scope of this chapter. Readers are referred to Lipska and Weinberger [11], for review.

In this chapter we will focus on another group of neurodevelopmental models with quite promising implications for schizophrenia: Lesion models. First, we will report on existing lesion models targeting different brain areas with implication in schizophrenia. Then, we will focus on the neonatal hippocampal lesion model, which possesses significant validity concerning modeling disease emergence, course, and core symptoms of schizophrenia. 


\subsubsection{Lesion models}

Based on the pathology observed in specific brain regions in schizophrenic patients, it became obvious to investigate whether disrupting these neuronal and functional circuitries would mimic clinical manifestations seen in schizophrenia. This idea was operationalized by damaging corresponding brain regions in adult rodents, and studying the direct effect on behavior and cognition of this disruption. There is a great deal of research done in the adult (i.e. at postnatal day 42) rodent brain. While first attempts in inducing a lesion were crude and relatively tissue unspecific (e.g. aspiration lesions), newer approaches became more precise with less damage to adjacent tissue. For instance, a widely used method to disrupt specific brain sites is the stimulation of excitatory glutamate release or the administration of direct glutamate receptor agonists (e.g. kainic acid), and thus producing relatively selective lesions, damaging predominantly pericaryal sites [115]. Electrolytic lesion techniques, on the other hand, destroy neuronal cell bodies as well as axons.

In the 70ties, scientists developed methods for selectively lesioning central norepinephrine and dopamine containing neurons in the neonatal rat brain. This was done by using 6-hydroxydopamine, an approach, originally applied in animal models of Parkinson's disease as well as in mimicking selfinjurious behavior in the context of the Lesh-Nyhan syndrome [116, 117].

Another approach addressed the transient, not permanent, inactivation of specific brain regions during a critical time window of brain development. Lipska and colleagues [118], used tetrodotoxin (TTX), a potent and specific blocker of the voltage-gated $\mathrm{Na}^{+}$-channels to inactivate the ventral hippocampus in neonatal rats. As its effect is completely reversible, this method does not result in permanent, vast damage of the brain.

\section{The prefrontal cortex (PFC)}

It is not surprising, that a region like the PFC with its subdivisions is one of the most intensively studied brain sites in schizophrenia [44, 85, 93, 119]. As mentioned earlier, abnormalities in structure as well as metabolism were consistently reported in human studies. Also cognitive functions, which arise from this brain area (e.g. organization of behavior, behavior monitoring, executive attention, inhibition and facilitation) are often reported to be compromised in schizophrenia patients [44, 89]. In the rat, medial and lateral PFC are considered as homologues of the primate dorsal PFC and orbital PFC, respectively. The medial PFC (MPFC) is implicated in working memory tasks. It has been hypothesized that the MPFC is primarily engaged in the temporary storage and processing of information lasting from milliseconds to several seconds [120]. Lesions of the rat adult MPFC result in 
various behavioral and neurochemical deficits also seen in schizophrenia, for example, increased responsiveness to dopamine agonists [121], decrease in haloperidol-induced catalepsy and initiation of stereotypic behavior [122], apomorphine-induced PPI deficits [123] as well as hypersensitivity to stress [124]. Fourteen days - but not 28 days - postoperatively, bilaterally ibotenic acid MPFC lesioned rats showed increased spontaneous locomotion as well as apomorphine-induced hyperactivity. Levels of noradrenalin in the cingulate cortex were reduced, while dopamine, DOPAC as well as homovanillic acid in the medial striatum were increased [124]. Also deficits in higher brain functions were apparent in MPFC lesioned animals. Ibotenic acid lesioned rats manifested symptoms resembling those of dysexecutive syndrome in humans. MPFC lesions resulted in disruption of decisional processes, as the performance of the lesioned rats was on a random level in a task addressing decision making [125]. In an analogue of the WCST, which is usually applied to assess executive functions in humans and widely used in schizophrenia research, the performance of electrolytically MPFC lesioned adult rats was impaired, while the LI effect was intact [126]. In a recent study comparing lateral and medial prefrontal cortical lesions in rats, lateral prefrontal lesions did not affect the behavior of the experimental group in any of the paradigms used in this study. On the other hand, an increase in PPI was reported in rats with medial prefrontal cortex lesions, while LI was not affected [127].

Lesions affecting the PFC with its subdivisions resemble somewhat the cognitive symptoms of schizophrenia. It was mentioned earlier, that the dopamine innervation in the MPFC is implicated in the regulatory inhibition of several dopamine terminal fields. Several lines of evidence propose that specifically the MPFC can influence subcortical dopamine [128-131].

The behavioral alterations seen in the studies discussed above go in concert with augmented mesolimbic and nigrostriatal dopamine activity [132135]. Treatments counteracting these effects (e.g. neuroleptics) reverse behavioral symptoms [132, 133]. Thus, lesions in the PFC have good predictive and face validity. However, the lack of evidence for a "lesion" underlying the behavioral and cognitive disturbances seen in schizophrenia presents a great obstacle for the construct validity. This is of course an aspect which also holds true for the following lesion models, addressing different brain regions.

\section{Thalamus}

The thalamus is a composite of multiple nuclei that relay and filter sensory inputs, a function which involves multiple corticostriatothalamic loops modulated by the cerebral cortex [for review, see ref. 136]. Based on 
human data, the thalamus and its nuclei moved into the scope of interest in schizophrenia research. Thalamic nuclei are involved in the regulation of PPI and sensorimotor gating [137], both are features affected in schizophrenia patients [35]. Furthermore, using magnetic resonance imaging, it was shown that lower relative glucose metabolism in the pulvinar was associated with more hallucinations and more positive symptoms, while lower relative glucose metabolism in the mediodorsal thalamic nucleus was associated with more negative symptoms in non-medicated patients [138]. Also other studies could replicate similar findings of reduced thalamic activity [139], pointing to deficits in filtering and information processing seen in schizophrenia patients [62]. Decreased activation within the whole thalamus, anterior nuclei and the medial dorsal nucleus were found using fMRI during performance of memory tasks in patients [140]. While functional findings seem to be consistent, structural findings are to a certain degree heterogeneous. Some post-mortem studies proved abnormalities in thalamic volumes in schizophrenia [141], while others failed to replicate this finding [142].

Among other thalamolimbic sites, the habenular complex is believed to be a key component in various physiological functions. It builds the dorsal diencephalic conduction system with both the stria medullaris and fasciculus retroflexus [143, 144] and serves as a link between the limbic forebrain and the midbrain-extrapyramidal motor system. The habenula was reported to be involved in some psychiatric disorders [144] including schizophrenia [145, 146]. Lesions of the habenula lead to a variety of behavioral alterations. Specifically, habenula-lesioned rats tested in a task measuring attention (Five choice serial reaction time task, 5CSRTT) demonstrated a time-dependant behavioral pattern. After the lesion, rats had an increase in premature, impulsive responding, which improved with time. Selective attention did not show deficits at the beginning, but deteriorated afterwards. Administration of amphetamine increased, while haloperidol decreased premature responding [147]. These findings serve as a link between attentional deficits in schizophrenia, limbic dopaminergic hyperactivity and potential structural abnormalities in the habenular complex. Additionally to thalamic structural abnormalities seen in schizophrenic patients [148], this finding is in some agreement with the deficit in attentional performance of affected individuals [149]. However, thalamus lesions often produce a syndrome of central pain and ataxia [for review, see ref. 150], symptoms not observed in schizophrenia. As with other lesion models, the construct validity is very limited.

In the context of subcortical lesion models, there are various studies looking at the effect of intracerebroventricular lesion using kainic acid [e.g. refs. 151, 152, 153]. Administration of kainic acid in the rat brain leads to an 
immediate as well as delayed neuronal cell death in the hippocampus and other regions of the medial temporal lobe. It was originally used as an animal model of neurodegeneration, implying potential relevance of these mechanisms for the pathophysiology of schizophrenia, too [153]. Intracerebroventricularly administered kainic acid produces an increase in locomotor activity in a novel environment. More grooming was also reported at the beginning of testing in lesioned rats [152]. Additionally, increased dopamine receptor binding in the nucleus accumbens has been reported [151], a finding, which was also observed in adult lesions of the hippocampus, that will be discussed below.

The apparent destruction and artificiality of this lesion type hamper drawing a link to schizophrenia, despite the resemblance of some symptoms. Nevertheless, this model results quite often in seizures [e.g. ref. 151] and unnecessary suffering of the animals, and should be regarded with caution.

\section{Hippocampus}

As mentioned earlier, the hippocampal formation gained a significant amount of experimental attention due to consistent findings in schizophrenia patients [86, 154] and because this brain site projects to the prefrontal cortex $[155,156]$ and participates in the regulation of the mesolimbic dopamine system [157]. In this section we will consider hippocampal lesions, again in the adult rat. Research has been focused predominantly on two parts of the hippocampal formation: Dorsal and ventral hippocampus (DH and $\mathrm{VH}$, respectively) with both leading to different phenotypes. Ventral hippocampal lesions have been reported to result in differential neurochemical pattern in cortical and subcortical areas, resembling core symptoms of schizophrenia. This site has been chosen as lesion target because it directly projects to the prefrontal cortex [158] and corresponds to the anterior hippocampus in humans, the region which shows structural abnormalities in schizophrenic patients [86]. Both lesions of the $\mathrm{DH}$ and the $\mathrm{VH}$ enhance various dopaminemediated behaviors, e.g. spontaneous locomotion, amphetamine-induced hyperactivity and stereotypy [122, 159, 160], while the behavioral effects observed were site specific. For instance, ibotenic acid lesions of the DH induced a transient increase in spontaneous locomotion, but not in amphetamine-induced hyperactivity [159]. On the other hand, VH lesion permanently affects both exploratory activity and amphetamine-induced hyperactivity [160]. Indeed, spontaneous activity following hippocampal lesion has been reported to be site specific [161] with permanent increase after ventral or combined dorsal-ventral lesions, but only transient increase after dorsal lesions. One reason for this finding might be that major hippocampal striatal projections arise in the ventral hippocampus and 
innervate ventral parts of the striatum [162]. Moreover, it has been proposed, that the dorsal hippocampus might affect striatal functions indirectly through its connections with the ventral part [163]. In a study comparing prefrontal cortical and both hippocampal (VH and $\mathrm{DH}$ ) lesions, rats with either MPFC or $\mathrm{VH}$ lesions showed reduced haloperidol-induced catalepsy, while $\mathrm{DH}$ lesions did have no significant effect. Also in the same study, DH as well as $\mathrm{VH}$ ibotenic acid lesions demonstrated less frequent oral stereotypies than control animals [122]. Both dopamine antagonist-induced catalepsy and dopamine agonist-induced stereotypies are believed to be largely dependent on postsynaptic dopamine-mediated mechanisms in the striatum [122].

The results shown in studies using adult hippocampal lesions, especially ventral lesions, have great resemblance with schizophrenia symptoms, predominantly addressing positive symptoms and dopamine-mediated deficits. Thus, adult hippocampal lesion models have both face and predictive validity.

The most limiting shortcoming of all lesion studies in general is the lack of observed lesions in schizophrenia. In particular, brain injuries in the adult do not lead to schizophrenia. However, they may give rise to various dopamine-mediated symptoms, which are observed in schizophrenia and can be reversed by neuroleptics. Structural abnormalities as well as neurologic soft signs in schizophrenia patients led to the idea, that there is an early developmental injury that remains relatively silent until adulthood [85, 164]. In this context, schizophrenia represents a unique case, as it was shown, that the degree of functional compensation increases with the postinjury interval (also called "Kennard principle" [165]). Schizophrenia represents the opposite process with successful functional sparing during short postinjury intervals (i.e. before adulthood) and deficient sparing during long postinjury periods (i.e. after puberty, adulthood) resulting in the emergence of the already described schizophrenia symptoms. Following this rationale, Lipska and co-workers - among others - suggested, that the effects of early (i.e. neonatal) hippocampal damage in limbic dopamine systems would not be attenuated by maturation, instead, it would emerge with maturation (the so called "schizophrenia principle" instead of "Kennard principle") [166]. The following section will therefore focus on a very influential animal model of schizophrenia: The neonatal ventral hippocampal lesion model.

\section{The neonatal hippocampal lesion}

As described above, adult lesions enhanced our understanding concerning mesolimbic and corticostriatal dopaminergic pathways. However, their heuristic value in modeling a broad spectrum of schizophrenia symptoms as well as disease emergence and progression is somehow limited. 
Following the procedure used in adult lesions, excitotoxic agents (e.g. ibotenic acid) were also applied in the neonatal lesion model. The main difference is the lesion time point. In this model, bilateral lesions to the ventral hippocampus were made on postnatal day 7 (PD 7), thus inducing an insult of the hippocampus, which disrupts the development of relevant cortical and subcortical circuitry in which the hippocampus participates. Again, the lesion targeted the ventral hippocampus, because it directly projects to the prefrontal cortex [158] and corresponds to the anterior hippocampus in humans, a region, whose relevance for schizophrenia was demonstrated [86].

The most intriguing aspect of this animal model is the postpubertal emergence of symptoms. Tested at PD 35, lesioned rats do not show significant behavioral aberrations. However, testing them at PD 56 (i.e. early adulthood), rats with $\mathrm{VH}$ lesion demonstrate symptoms similar to psychotomimetic models discussed earlier as well as to schizophrenia [166]. Providing that results of this model have been reviewed repeatedly and intensively elsewhere $[10,11,108]$, we will focus on some predominantly behavioral features of this model with high face validity.

Similar to sensitization to psychostimulants, neonatally lesioned rats show at PD 56, but not at PD 35, increased activity as response to novelty as well as after amphetamine administration. These symptoms were reversed by haloperidol. At PD 70, neonatally lesioned rats display markedly increased activity after stress [166]. The changes in the reported behaviors are believed to be primarily related to increased mesolimbic and nigrostriatal dopamine. It has also been reported, that lesioned rats showed no differences in haloperidol-induced catalepsy when tested on PD 35, while apomorphine administration increased locomotor activity at this time point. Contrary to that, as young adults (PD 56), lesioned rats showed reduced catalepsy following haloperidol, while the apomorphine-induced hyperactivity was still apparent [167]. Interestingly, clozapine (as an atypical antipsychotic) as well as haloperidol (typical antipsychotic) could suppress hyperlocomotion [168], but clozapine was not effective for reversing social deficits [169].

Other studies demonstrated that neonatally ventral hippocampal lesioned rats have altered dopamine release in the striatum, nucleus accumbens and in the frontal cortex in response to mild repeated stress as well as after chronic haloperidol administration. This manifested itself in a modest reduction in baseline striatal dopamine release, which became more pronounced after chronic stress [170]. Contrary, chronic haloperidol treatment resulted in increased dopamine release in the same regions.

Concerning motivational and reward related behaviors, which are involved in the negative symptoms in schizophrenia, some studies reported 
deficits in reward mechanisms [171], reduction in saccharine preference as well as deficit in amphetamine-induced conditioned place preference only in neonatal VH lesion, not in adult lesion [172]. Also there are various findings supporting the face validity of this model, as they show social deficits resembling those seen in schizophrenia.

The observed decrease in social interaction time [173] was increased by clozapine in some studies [174], but not in others [169]. It has also been shown, that social memory is impaired and that haloperidol was partially effective in attenuating this symptom [175]. It is intriguing, however, that social deficits were observed at both PD 35 and PD 65, although hyperactivity was only present after puberty [169]. Interestingly, deficits in spatial memory were also observed with no time delay. They were present at PD 25, 40 and 80 [176]. These findings resemble the clinical picture seen in schizophrenia, with negative symptoms, e.g. social withdrawal, and some cognitive deficits existing already long before the onset of positive symptoms.

Also, deficits in working memory and functions which require the functional integrity of the PFC have been demonstrated in this model. For example, in a study using T-maze, two related working memory tasks were applied: 1 . The continuous delayed alternation and 2. The discrete paired-trial delayed alternation task [177]. Lesioned rats required longer time to learn the former, while working memory was shown to be compromised in the latter. Furthermore, deficits in sensorimotor gating and attentional processes have also been reported in neonatal VH lesioned rats $[178,179]$. Consistent with other studies [180], there was no difference in startle response, but there were deficits in PPI which were reversed by atypical, but not typical, antipsychotics [179]. The reported behavioral phenotype seems to be mediated by genetic influence, which might attenuate or enhance the susceptibility to the VH lesion effects [181].

Interestingly, these symptoms could not be explained by the boost of gonadal hormones in puberty, as the absence of these by means of castration did not prevent the emergence of behavioral disturbances in adulthood. On the contrary, it enhanced those linked primarily to the mesolimbic dopamine system [182]. Furthermore, it has been shown that removal of PFC neurons in adult animals with neonatal lesions can restore some of the behavioral alterations [183]. This suggests that this behavioral phenotype is due to abnormal or dysregulated PFC activation resulting in increased subcortical firing upon dopaminergic subcortical stimulation.

Concerning molecular and electrophysiologic aspects, a considerable amount of research suggests that abnormal cortical interaction between the neurotransmitter systems dopamine, glutamate and GABA may give rise to 
cortical dysfunction in the neonatally $\mathrm{VH}$ lesioned rats. Many of these findings have been observed in schizophrenic patients, e.g. reduced glutamate decarboxylase-67 (GAD-67) expression, reduced BDNF expression and changes in neuronal morphology [for overview, see refs. 108, 184, 185].

Interestingly, it has been shown that neonatal $\mathrm{VH}$ lesions lead to prominent and widespread cell death even in brain areas distant from the lesion site, while cell death is restricted to the lesion area in the analogous adult model [186].

Unlike pharmacological models, the neonatal VH lesion model affects other neuroanatomic and functional systems outside the hippocampus and elicits a unique time-dependent behavioral and physiological phenotype reminiscent of schizophrenia. As was mentioned before, this model possesses good predictive as well as face validity. Many symptoms are reversed by atypical antipsychotics resembling treatment effects observed in clinical populations. As was discussed earlier in this chapter, newer studies based on this model also apply transient, not permanent, "lesions”. Using tetrodotoxin makes the transient inactivation of the $\mathrm{VH}$ during a critical period of neurodevelopment possible [187]. The behavioral phenotype induced by this model is similar to that of the permanent lesion, however, the magnitude is smaller compared with those observed after the excitotoxic lesion. Moreover, the reversible hippocampal disconnection does not lead to significant social deficits as seen in the permanent model [118].

To sum up, adult and neonatal lesions discussed in this section produce symptoms relevant for schizophrenia, while the neonatal lesion model has more heuristic value and resembles the clinical course and temporal pattern as well as the symptoms of the disease. Table 2 illustrates the phenotype of both adult and neonatal lesion models and compares their main corresponding findings.

As it was portrayed in this section, lesion models, whether in adult animals or in the neonate, enhanced our understanding concerning putative temporal as well as physiologic mechanisms of relevant features of schizophrenia. They filled the gap, which was present in pharmacological models and illustrated a potential mechanism underlying the delayed emergence of psychotic symptoms. Compared to direct pharmacological manipulation models, lesion models accounted for many schizophrenia relevant symptoms. However, the most prominent shortcoming in these models is their artificial character in inducing a "lesion". Recent developments are still based on the idea of early brain injuries, which might be induced by different toxins or other hazardous agents. These might act directly on the brain (neurotrauma, brain contusion, viral infections, malnutrition, etc.) or indirectly via psychological mechanisms (e.g. psychosocial stressors and psychotrauma). But this is not the only possibility 
Table 2. Comparison between the phenotype of adult versus neonatal bilateral ventral hippocampal lesion in the rat.

\begin{tabular}{|c|c|c|}
\hline Domain & $\begin{array}{c}\text { Adult lesion } \\
\text { (at postnatal day 42+) } \\
\end{array}$ & $\begin{array}{c}\text { Neonatal lesion } \\
\text { (at postnatal day 7) }\end{array}$ \\
\hline $\begin{array}{l}\text { Hypersensitivity } \\
\text { to stress }\end{array}$ & $\begin{array}{l}\text { Adult lesion does not lead to } \\
\text { hypersensitivity to stress } \\
\text { (i.e. no increased activity } \\
\text { after saline injection or after } \\
\text { swim stress) [160] }\end{array}$ & $\begin{array}{l}\text { Neonatal lesion leads to } \\
\text { hypersensitivity to stress and } \\
\text { resembles in this respect the adult } \\
\text { lesion of the medial prefrontal } \\
\text { cortex [124] }\end{array}$ \\
\hline $\begin{array}{l}\text { Haloperidol-induced } \\
\text { catalepsy }\end{array}$ & $41 \%$ decrease of catalepsy & 51\% decrease of catalepsy [167] \\
\hline $\begin{array}{c}\text { Apomorphine-induced } \\
\text { hyperactivity }\end{array}$ & \multicolumn{2}{|c|}{ Both lesions lead to apomorphine-induced hyperactivity $[167,172]$} \\
\hline $\begin{array}{c}\text { Apomorphine-induced } \\
\text { stereotypy }\end{array}$ & Reduced stereotypic responses & \begin{tabular}{|c} 
Potentiation of stereotypic responses \\
{$[167]$} \\
\end{tabular} \\
\hline Memory & No memory impairment & Memory impairment [177] \\
\hline Reward sensitivity & No deficit & Deficit in reward sensitivity [172] \\
\hline $\begin{array}{l}\text { Cellular and neuronal } \\
\text { pattern of lesion }\end{array}$ & $\begin{array}{l}\text { Cell death is restricted to the } \\
\text { ventral hippocampal area }\end{array}$ & $\begin{array}{l}\text { Widespread and prominent cell } \\
\text { death, even in sites distant from } \\
\text { the lesion area (striatum, } \\
\text { nucl.accumbens, cortical areas } \\
\text { surrounding the VH) [186] }\end{array}$ \\
\hline
\end{tabular}

for inducing a lesion. Indeed, recent models are guided by the notion of "genetic lesion". In other words, the observed phenotype in conventional adult and neonatal lesion models might be produced not by means of mechanical induction, but, due to dysfunctions in neuronal maturation, migration and/or differentiation. Providing that schizophrenia has a strong genetic background and due to the recent vast development and advance in genetic engineering and technology, present models focus on the effects of some candidate genes, which have been shown to be involved in the pathophysiology of schizophrenia in population studies. These models are the topic of the next section.

\section{4. "Present"}

\subsection{Genetic models}

Schizophrenia is a highly heritable disease. The population incidence is $0.17-0.57$ per 1000 , and the point prevalence is $2.4-6.7$ per 1000 reflecting the typical disabling chronic clinical course of the disease [188]. Using family, twin and adoption studies, it has been demonstrated, that the morbid risk of schizophrenia in relatives correlates with the degree of genes shared [189]. In contrast to lifetime morbid risk in general population of $1 \%$ [190], the incidence of schizophrenia is $6 \%-17 \%$ in first degree relatives 
(e.g. parents, siblings) of an individual with schizophrenia. In monozygotic twins, who have $100 \%$ of their genes in common, the morbid risk increases to approx. 50\%. Notably, even in this case, where the genetic background is identical, the risk to be affected is - fortunately - not fully determined. This fact gives credence to the role of non-genetic factors, e.g. environmental and social influences. Interestingly, the risk estimations as well as the emergence of schizophrenia seem to be universal [191] and fairly consistent across different racial groups [192].

The main focus of present schizophrenia research is set on the elucidation and determination of genetic factors increasing susceptibility to the disease. Studies in the last two decades have demonstrated that schizophrenia is not a monogenic, unifactorial disease. There is almost uniform agreement on the involvement of non-Mendelian mode of inheritance. The prevailing notion implies polygenic transmission with interacting environmental factors [189]. New research approaches aim at identifying genetic markers, which significantly co-exist with the disease in a relatively diagnosis-specific manner within affected families (i.e. genetic linkage studies) or within populations (i.e. genetic association studies) [193].

A great body of clinical and basic research evidence shed light on the putative role of some susceptibility genes for schizophrenia. Indeed, it has been an enormous amount of genetic linkage and whole genome scan studies accumulating for the last twenty years. Some of which has been evaluated in large meta-analysis studies [194, 195].

The vastly increasing advances in genomics and gene technologies facilitated the investigation of the genetic underpinnings of complex behavior. In contrast to the leading part of the rat in the past, the laboratory mouse settled into the center of scientific attention as being the most important model organism in the study of psychiatric genetics. This is not only due to the biological and physiological homology in wide parts of brain organization as well as the numerous features of behavioral repertoires mice and humans share, but also to practical issues (e.g. efficiency in breeding, economical maintenance, space saving due to small body size, etc.) that have contributed to the wide popularity of the laboratory mouse as a main modeling organism for molecular psychiatric research [196]. Besides, mouse and human genomes have been reported to share many similarities [197].

Improved and precise methods in gene targeting, conditional knock-out and transgenic technologies [198] as well as targeted mutations paved the way for studying and modeling complex issues like depression, circadian rhythm, narcolepsy, aggression, social interaction and stress-related behaviors [199, 200] in the mouse. Indeed, current methods open the opportunity for generating mutant mice carrying virtually any given gene of 
interest [196]. This development was also supported by the increasing understanding of molecular and cellular mechanisms in concert with genetic influences in modulating sophisticated functions, e.g. cognition.

The availability of the Mouse Genome Database, which unites genomic and phenotypic information about the laboratory mouse and contains (up to September 2007) approximately 29.000 sequenced genes [201] and the vast sequencing of the mouse genome [202] accelerated the generation of diverse mouse models of psychiatric disorders, including schizophrenia, all over the world. However, despite the optimistic perspective evolved from current technical advances and the fast increasing efforts to complete the mosaic of the genetics of schizophrenia and mental disorders, developments in this field have soon posed various further problems. These could be divided in two groups: First, methodological considerations inherent in genetic modeling of mental disorders and addressing cognitive functions in mice and, second, the nature of clinical diagnosis of schizophrenia including the lack of objective and clear-cut markers of the disease.

In addition to disease heterogeneity and the lack of objective stateindependent markers, the plurivalent outcomes of genetic modifications as well as the imperfect relation between genes and behaviors underline the necessity of quantifiable, detectable and reliable disease markers. In this context, studies of biological markers of psychiatric disorders have yielded an overwhelming amount of ambiguous biological and physiological indicators, many of which are not disease-specific and tend to confound with other physiological parameters. Moreover, these often single findings have limited reproducibility both among and within patients [203]. Taking this into consideration, the definition of "endophenotypes" (i.e. intermediate phenotypes) presents a useful tool for diagnosing and studying the genetic mechanisms in mental disorders [204]. According to Gottesman and Gould [205], endophenotypes have to fulfill the following criteria to be called such: (1) The endophenotype is associated with illness in the population. (2) It is heritable. (3) It is state-independent (i.e. it is detectable in the affected individual whether or not the illness is active). (4) Endophenotype and illness co-segregate within families. (5) It is found in affected as well as in unaffected family members to a higher rate than in the general population.

In the previous sections some putative endophenotypes have been discussed. For example, sensorimotor gating (usually measured by means of prepulse inhibition) and working memory tasks are believed to be endophenotypic behavioral and cognitive domains, respectively. Additionally, hippocampal size and morphology as well as frontal lobe volume and activity have consistently been implicated in schizophrenia and fulfill the above mentioned criteria for endophenotypes [for review, see refs. 205, 206]. 
Giving the fact, that genes affect cognitive functions and behavioral features [for example frontal functions, ref. 207], it is not surprising that various such genes have been discovered and found to be altered in schizophrenia patients [e. g. ref. 208]. As noted previously, such candidate genes have been successfully linked to the disease, even though they are not necessarily disease-specific. Their physiological roles and functions are pleiotropic, e.g. affecting different neuronal circuitry and interacting with various neurotransmitter systems.

The first genetic mouse models of schizophrenia were based on disrupting dopaminergic and glutamatergic neurotransmitter systems, continuing the study of dopamine and glutamate hypothesis of schizophrenia. Thus, these models were again set on the assumption that the schizophrenic phenotype arises from a disturbed neurotransmitter function. Since schizophrenia is not the result of disruption of a single neurotransmitter system, these models had essentially the same limitations as pharmacological models, even though many of them reliably mimic schizophrenia symptoms [6, 209, 210].

For the sake of brevity, we will not discuss all genes that have been implicated in schizophrenia. Instead, we will focus on six candidate genes (i. e. genes that have a neurobiologic role in signal transduction, neuronal maturation, neurite outgrowth and/or neurotransmission and are localized within putative disease-linked regions): Neuregulin1 (NRG1), Complexin 2 (CPLX2), Disrupted-in-schizophrenia 1 (DISC1), Catechol-O-methyl transferase (COMT), Regulator of G-protein signaling 4 (RGS4) and Dystrobrevinbinding protein 1 (DTNBP1). As noted previously, the list of candidate genes of schizophrenia is growing daily. However, associating a gene with the disease in a small number of studies does not automatically qualify it for being called "candidate gene". In addition, not all genes linked with the disease seem to be biologically plausible, as they have no overt role in the pathophysiology.

Thus, the genes selected in this chapter unify some aspects: (1) They have been repeatedly and consistently reported to be involved in the disease. (2) They seem to have a biologically plausible overt role. Consequently, modeling them in mice might provide good construct validity. (3) Some of them have been implicated in cognition. (4) Studies in which they are disrupted delivered promising potential in terms of face and predictive validity.

In one case, complexin 2, evidence of being a schizophrenia candidate gene in humans or mouse models is somehow sparse. It may ultimately turn out to be a non disease-specific risk gene modulating the disease phenotype [211]. In this context, a great part of work in our lab is currently focusing on it. As we do not attempt to give an overview of the enormous and steadily 
.

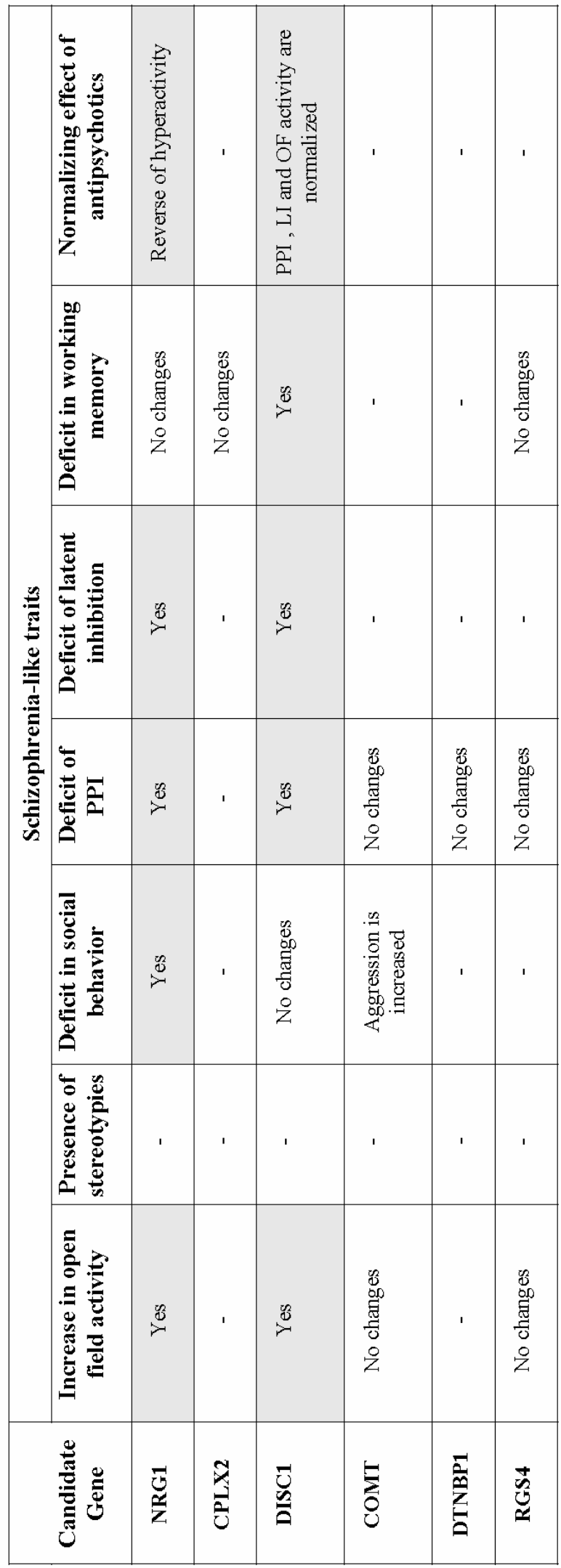


growing amount of literature on genetic mouse models of schizophrenia, we recommend to the reader recent reviews providing an overview of other promising genes [212-214].

In the following section, the above mentioned genes will be discussed. First, their relevance for schizophrenia as well as their main physiological features and roles will be addressed. Then, evidence concerning their endophenotypes will be presented, where applicable. Finally, data are given on respective mouse models regarding the domains locomotor activity, stereotypy, social behavior, sensorimotor gating, latent inhibition, memory and effect of antipsychotic administration. Table 3 provides an overview of the behavioral phenotype of mice, mutant for the here presented candidate genes of schizophrenia.

\subsubsection{Neuregulin 1 (NRG1)}

NRG1 was identified as being a susceptibility gene for schizophrenia in a genome-wide linkage scan study of 33 Icelandic families with schizophrenia [22]. The systematic study of the locus 8p21-22 revealed association between the disease and a multi-marker haplotype at the 5' end of NRG1 gene [22]. This has been confirmed in other samples [215, 216], although allelic heterogeneity was present in many cases [217-219]. Several meta-analyses have supported the association between NRG1 and schizophrenia [220-222] and diverse studies have been recently reviewed elsewhere [223]. However, as often observed in genetic studies of mental illness, negative results claiming non-association have also been found [224-226]. Notably, it has recently been reported that NRG1 shows extreme population differences in allele and haplotype frequencies [227]. Taking this into account, conflicting results from different populations should not be surprising.

Neuregulin 1 belongs to the family of growth and differentiation factors. Through its interaction with the ErbB tyrosine kinase transmembrane receptors it induces differentiation and growth of neuronal, glial and other cell types. NRG1 gene is a huge, complex gene that spans more than 1.1 megabase and produces diverse isoforms through alternative splicing. For an extensive discussion on the NRG1-ErbB signaling mechanisms, the reader is referred to [228, 229], for diverse physiological roles of NRG1 to [230, 231].

Further evidence points to NRG1 signaling involvement in PFC physiology and neurotransmission [232]. For instance, ErbB4, a crucial NRG1 receptor, is localized at GABAergic terminals of the PFC [233] which is, as we noted previously, one of the most studied brain sites involved in schizophrenia. Furthermore, a recent study has demonstrated, that altered NRG1/ErbB4 signaling results in NMDA receptor hypofunction in schizophrenia [234]. Additionally, ErbB4 is implicated in activity-dependent 
maturation and plasticity of synaptic structure. Thus, genetic defects in NRG1/ErbB4 signaling might lead to glutamatergic hypofunction [235], another line of evidence strengthening a role for NRG1 as a susceptibility gene for schizophrenia.

Even though the NRG1 gene is one of the most promising candidate genes studied for schizophrenia, its cognitive and clinical correlates have received relatively little attention. A number of studies elucidate its putative involvement in cognitive dysfunction in schizophrenia. The study by Hall and colleagues [236] showed an association between a risk-allele within the NRG1 gene (SNP8NRG243177), which is part of the risk-associated haplotype “deCODE” [22, 237], and decreased activation in frontal and temporal brain regions, increased development of psychotic symptoms and lower premorbid IQ.

A recent MRI study proved the association between the Icelandic at-risk haplotype $\left(\mathrm{HAP}_{\mathrm{ICE}}\right)$ with lower hippocampal volumes in schizophrenia patients and their non-affected relatives [216]. Hippocampal volume reductions have been widely replicated in schizophrenia patients and their unaffected family members, for example in twin studies [238, 239]. As mentioned previously, hippocampal volume and size are believed to be consistent endophenotypic markers for schizophrenia and other mental disorders. Interestingly, children who carry the at-risk variant of the NRG1 gene have significantly larger grey and white matter volumes, independently of whether they were affected by childhood-onset-schizophrenia (COS) or not. However, COS risk allele carriers demonstrate a steeper rate of subsequent brain matter volume decline into adolescence [240]. These findings support a role for the NRG1 gene in defects of neurodevelopment in schizophrenia.

Considering mouse data, genetic inactivation of the NRG1 gene in mice is associated with abnormalities in several types of schizophrenia-relevant behavior. Thus, spontaneous locomotor activity in the open field is increased in NRG1 mutants [22, 241]. Moreover, the atypical antipsychotic clozapine reduces this hyperactivity [22]. NRG1 mutants also show a selective impairment in response to social novelty [242], which is similar to social deficit observed in schizophrenia patients. Some behavioral tests related to cognitive deficits in schizophrenia were altered in NRG1 mutant mice. Prepulse inhibition, which is believed to be a test for sensorimotor gating [37], is impaired in NRG1 mutant mice [22]. Latent inhibition, which - with certain limitations - might reflect selective attention [39], is also disrupted in NRG1 mutant mice [243]. Contrary, other cognitive processes such as spatial learning and working memory appear to be intact in NRG1 knock-out (KO) mice [242]. 
Taken together, clinical evidence as well as data from mouse models on NRG1 support its candidacy as a susceptibility gene for schizophrenia. NRG1 knockout mouse models deliver fairly good construct and face validity. Pharmacological interventions using antipsychotics have also proved to reverse the schizophrenia-like symptoms in this knockout mouse model and thus, predictive validity of this line of research is quite satisfying. However, the pleiotropic role of the NRG1 gene in neurodevelopment and neural plasticity complicate the understanding of its role in cognition and schizophrenia.

\subsubsection{Complexin 2 (CPLX2)}

In contrast to the other candidate genes presented here, which have been repeatedly and intensively studied, complexins are just starting to move in the focus of scientific attention regarding schizophrenia. Complexins are presynaptic proteins, which play a key role in the modulation of neuronal exocytosis. They form a family of four small (134-160 residues) charged proteins, which are subdivided into two subfamilies: CPLX1/2 and CPLX3/4 [244]. CPLX1 is specifically expressed in the central nervous system. CPLX2, on the other hand, was detected in other, non-neuronal tissue [245]. CPLX3/4 are predominantly expressed in the ribbon synapses of the retina [244].

Various studies reported decreased CPLX2 mRNA and protein levels in postmortem hippocampal tissue of schizophrenia patients [246, 247]. As discussed previously, there is no doubt about the central role of the hippocampal formation in schizophrenia. Cause and significance of these findings regarding CPLX2 is still unclear. CPLX2 may not play the role of a typical candidate gene for schizophrenia but rather be a contributor/modifier of disease progression/severity. As such, it obviously also plays a part in diseases as different as Alzheimer disease, alcoholism or Huntington's Disease [211].

Few behavioral data exist on mice carrying a targeted deletion of the complexin 2 gene. For example, spatial learning and memory are not affected in CPLX2 mutant mice [248]. However, CPLX2 KO mice are more vulnerable to maternal deprivation stress in comparison to wild type control animals [248]. Two other studies have demonstrated electrophysiological alterations, which are not directly related to a schizophrenia phenotype. However, it has been shown that LTP in CA1 and CA3 in the hippocampus is reduced in CPLX2 KO [249]. Further characterization of schizophrenia related behavior of CPLX2 mutant mice is required. To conclude, complexin 2 appears to be an interesting gene in the context of schizophrenia, albeit more in the sense of a non-specific disease modifier. 


\subsubsection{Disrupted-in-schizophrenia 1 (DISC1)}

DISC1 gene is one of the most intensively studied candidate genes for schizophrenia. There is a great body of evidence pointing to its association with major psychiatric disorders [250], e.g. bipolar disorder, schizophrenia [251] and the autism spectrum [252]. DISC1 was first identified and located at the breakpoint of a balanced chromosomal translocation $\mathrm{t}(1 ; 11)(\mathrm{q} 42.1$; q14.3) in a large Scottish family which displayed a variety of psychiatric disorders [253]. The breakpoint at 1q was cloned and it was shown that it includes two genes, called DISC1 and DISC2 [254].

Several association and linkage studies in different populations [see also ref. 250 for review] supported its candidacy for the involvement in psychiatric disorders. Many studies have shown associations between allelic variations in DISC1 and risk to develop schizophrenia. For example, it has been reported that a single three-locus haplotype within the DISC1 gene was over-transmitted to individuals affected with schizophrenia in a family-based association study in a Finnish population [255]. In addition, overrepresentation of five SNPs in the gene in schizophrenia patients has also been reported [256].

DISC1 is a complex gene, whose involvement in and implications for development and neuroplasticity are still poorly understood. It has 13 exons spanning over 200kb. The protein it encodes (consisting of 854 amino acids) is associated with various cytoskeletal proteins involved in centrosomal and microtubule function, cell migration, neurite outgrowth, membrane trafficking of receptors and possibly mitochondrial functioning [212, 257]. Additionally, a role in regulating adult neurogenesis has recently been reported [258]. In the human brain, DISC1 is expressed in cerebral cortex and hippocampus [259], both regions implicated in schizophrenia.

In the mouse brain, DISC1 is expressed from embryonic day 10 on to adult age, pointing to its role in neurodevelopment [260]. DISC1 interacts with many other proteins, including the nuclear distribution element-like 1 (NDEL1) protein, the nuclear protein lissencephaly 1 (LIS1), the fasciculation and elongation protein zeta-1 (FEZ1) and phosphodiesterase 4B (PDEB4). Interestingly, significantly reduced expression of NDEL1, FEZ1 and LIS1 in the hippocampus and DLPFC of brains from schizophrenia patients has been reported, while DISC1 expression remained unaltered [261].

There is a strong body of clinical [262] as well as molecular evidence linking DISC1 with different cognitive and behavioral phenotypes relevant to schizophrenia [250 for review]. The molecular link emerged from the interaction of DISC1 with PDEB4 [263]. PDEB4 belongs to the family of phosphodiesterases which are orthologous to the Drosophila learning and 
memory gene dunce [264]. Notably, phosphodiesterases play an imperative role in inactivating cAMP, which is a key signaling molecule implicated in learning and memory [for review, see refs. 265, 266].

Thus, alterations in this gene, as was shown from human studies, have deleterious effects on learning and memory. Mice carrying a point mutation leading to conversion from leucine to proline at residue 100 (L100P) of final DISC1 protein were shown to be abnormal in many types of schizophrenia related behavior tests [21]. Thus, DISC1 mutants show increase in locomotor activity in open field, deficit in prepulse inhibition and latent inhibition. Interestingly, all these alterations were sensitive to clozapine treatment [21]. DISC1 mutants also show deficit in working memory. However, social behavior of these mice was unimpaired [21].

Taken together, the DISC1 gene has proven to be a candidate gene for schizophrenia. DISC1 mutant mouse models demonstrate good predictive and partially also face validity. The construct validity is again a difficult issue, because schizophrenia is not a monogenic disorder. However, the involvement of DISC1 in the disease seems to be quite unequivocally accepted, although it is still unclear how alterations in the DISC1 gene can contribute to the emergence of the disorder.

\subsubsection{Catechol-O-methyl transferase (COMT)}

The gene encoding the enzyme catechol-o-methyl transferase (COMT), located at the chromosomal region 22q11, has been implicated in schizophrenia, bipolar disorder, anorexia nervosa, anxiety and other mental disorders [267, 268]. Its putative involvement in schizophrenia is quite apparent, due to its key role in dopamine catabolism. COMT has also been intensively studied in the context of the microdeletion syndrome involving its chromosomal locus (velo-cardio-facial syndrome), in which a high prevalence of psychotic and affective symptoms has been reported [269]. Many studies have been conducted focusing on a functional polymorphism that substitutes a valine to methionine at codon 158. This constellation influences enzymatic activity and consequent dopamine metabolism. Homozygous carriers for the Met allele have the lowest activity, resulting in reduced dopamine degradation. This allele has been shown to be associated with PFC activation [270] and improved performance in frontal cortical tasks [271]. Conversely, the Val allele confers higher activity and has been relatively consistently linked to reduced performance in PFC-related tasks [271, 272].

As with the other previously mentioned genes, COMT studies have delivered mixed results. While some studies have supported the association between Val/Met polymorphism and schizophrenia [273, 274], other 
studies found no association [275, 276]. Conflicting results emerged also from meta-analyses [277, 278] pointing to only a small effect of the COMT allelic variation on schizophrenia risk. Concerning the neurocognitive phenotype of the Val/Met polymorphism, there is a growing number of studies showing COMT involvement in PFC related functions [for review, see refs. 271, 279-282].

According to Craddock and colleagues [283], it cannot be ruled out, that a variation elsewhere in the COMT or neighbouring genes, such as ARVCF, is basis of the susceptibility to schizophrenia. Neither the mechanisms of how COMT regulates cognition nor its candidacy as a vulnerability gene have yet been clarified. The same conflicting pattern of evidence is found in mouse models of this gene. Mice lacking COMT did not show a prominent behavioral phenotype.

Thus, except for an increase in aggression, these mice show no changes in schizophrenia related types of behavior. The PPI and locomotor activity in open field are not affected in COMT mutants [20]. In conclusion, despite its apparent role in the physiology of the dopaminergic system and its potential implication in dopamine-mediated cognitive functions, the role of COMT in the pathophysiology of schizophrenia is still obscure.

\subsubsection{Dystrobrevin-binding protein 1 (DTNBP1)}

A considerable body of evidence supports the involvement of the gene encoding dystrobrevin-binding protein 1 (DTNBP1) - synonym: Dysbindin1 in the etiology of schizophrenia [284-286]. Nevertheless, some studies in diverse populations failed to find associations between schizophrenia and certain haplotypes of SNPs in the DTNBP1 gene [287-289]. DTNBP1 gene is located on chromosome 6p22.3 and encodes a 40-50 kDa protein that binds both $\alpha$ - and $\beta$-dystrobrevin. Both are members of the dystrophin glycoprotein complex [290], which is concentrated at postsynaptic densities in different brain areas.

DTNBP1 mRNA has been found to be expressed in diverse brain sites, including frontal and temporal cortices, nucleus accumbens, thalamus, amygdala and hippocampus. The reduction of DTNBP1 mRNA in multiple layers of the DLPFC in schizophrenia patients [291] as well as a selective decline in DTNBP1 expression presynaptically in the hippocampus support DTNBP1 as a candidate gene for schizophrenia [292]. Additionally, Talbot and colleagues [292] have reported an inverse correlation of DTNBP1 levels with the vesicular glutamate transporter 1 (VGluT-1) in the hippocampus of schizophrenia patients. This finding may support the involvement of this gene in schizophrenia. As noted earlier in this chapter, one of the original hypotheses on schizophrenia pathophysiology is the 
glutamatergic hypothesis, pointing to a glutamatergic hypofunction as basis of clinical features [293].

Neuropsychological data underline the putative function of DTNBP1 in cognitive deficits reported in this disease. Several studies succeeded in identifying high-risk haplotypes in the DTNBP1 gene [e. g. 294, 295] as well as in linking these with specific clinical features of the disease. For example, carriers of a high-risk haplotype were more likely to manifest high levels of negative symptoms in an Irish study of high-density schizophrenia families [296]. Another study reported that a variation in DTNBP1 is associated with negative symptoms [297]. These findings are of great interest in light of previously discussed effects of glutamatergic antagonists on inducing negative symptoms in healthy individuals [69]. Taken together, these data suggest DTNBP1 to be involved in mechanisms related to affect and mood domains of schizophrenia.

Different studies have reported relations between variants in DTNBP1, poor premorbid functioning and childhood-onset-schizophrenia (COS) [298], higher cognitive decline [299] as well as deficits in early visual processing [300]. Moreover, DTNBP1 variants have been reported to be involved in frontal brain functions in healthy subjects [301].

Despite promising evidence from clinical and basic research, DTNBP1 does not seem to be the ideal "schizophrenia candidate" gene. Disruptions in the dystrophin-associated protein complex result in Duchenne muscular dystrophy [302]. A murine spontaneous mutation in the DTNBP1 gene leads to Hermansky-Pudlak syndrome type 7 (HPS7), which is characterized by oculocutaneous albinism, prolonged bleeding and pulmonary fibrosis [303]. The sandy mouse (sdy) is a rodent model of HPS7. In this context, no schizophrenia-related behavioral features have been reported. This might be seen as a drawback for the candidacy of DTNBP1; however, considering conflicting human data as well as the complexity of schizophrenia pathophysiology and clinical manifestation, it should not be too surprising that alteration in this gene in a model organism like mice does not lead to an apparent phenotype, which satisfies validity criteria.

\subsubsection{Regulator of G-protein signaling 4 (RGS4)}

RGS4, which is localized at the chromosomal locus 1q23, is a particularly interesting candidate gene for schizophrenia, as clinical and basic research data show its biological implication in the disease. Also, this gene appears to be a target of the physiological response to environmental stimuli (e.g. stress). Stress in turn is regarded as one crucial factor for the emergence of psychiatric disorders. 
According to Levitt and colleagues [304], there are principally three lines of evidence supporting the candidacy of RGS4: First, genetic studies showing the association between schizophrenia and non-functional polymorphisms in the RGS4 gene [305-307]; second, decreased RGS4 expression in cortical regions in schizophrenia patients in a disease-specific pattern [308] and third, the differential regulation of RGS4 in the brain and its responsiveness to chronic stress [304, 309].

Although many studies have succeeded in associating RGS4 with schizophrenia [306] other studies conducted in different populations could not fully support this association [310-312]. Possible reasons for these conflicting results might be diagnostic differences between samples, heterogeneous and small sample sizes and/or locus heterogeneity. However, the biological and cellular role and function of RGS4 gene make it a very attractive and promising candidate. RGS4 was one of the earliest studied members of the RGS (regulators of G-protein signaling) family, which has been shown to be a highly diverse protein family. Regulator of G-protein signaling control the duration and regulate the timing of intracellular signaling of many G-protein mediated receptors [305]. The expression of the RGS gene family is widespread through the human body. Many of them show a distinct expression pattern in the rodent brain [313].

RGS4 mRNA has its highest levels of expression in frontal cortical regions, while subcortical sites (e. g. thalamus, striatum) manifest the lowest expression levels in normal postmortem human brain samples [314]. The regional density pattern in rodents seems to differ from that observed in humans [313]. It has been reported that RGS4 is linked to dopamine receptor D2 functions $[315,316]$ and inhibits signaling of metabotrobic glutamate receptors [317].

Concerning implication in neurocognitive functions, altered expression of RGS4 mRNA in the superior temporal gyrus (STG) in brains of schizophrenic patients has been documented [318]. The STG is believed to be a key region for speech and communication. Furthermore, it is connected to limbic regions, associated with schizophrenia pathology, and has been reported to be involved in the emergence of auditory hallucinations [319]. Another study demonstrated the impact of a variation at a RGS4 SNP, associated previously with psychosis (rs951436), on frontoparietal and frontotemporal regions during a working memory task and on grey matter structural connectivity as well as on white matter volume in healthy volunteers [320].

Concerning mouse behavioral data, RGS4 deficient mice did not display deficits in PPI [321]. RGS4 mutants also failed to show abnormalities in locomotor activity in open field, working memory in T-maze or Pavlovian 
fear conditioning [321]. Again, as noted previously, genetic variation in a specific gene in humans does not inevitably lead to comparable behavioral and clinical manifestations in mice. Nevertheless, the potential contribution of this gene to the evolvement of the schizophrenic phenotype is worthwhile further investigating. Regarding validity issues of the RGS4 mouse model, the construct validity might be considered satisfying due to aspects mentioned above. However, further research is needed for clarifying face and predictive validity issues.

Table 3 contains an overview summarizing the behavioral phenotype of mice mutant for the here discussed genes. As noted previously, we have only included studies yielding positive results regarding schizophrenia-related symptoms. There is an enormous amount of studies addressing various genetic alterations in the mentioned genes. However, the number of schizophrenia-relevant phenotypes is still limited.

\subsection{Present genetic animal models: Promises and problems}

Based on human data and experimental results with rodents, evidence is available for a role in schizophrenia of all the genes discussed here, be it as disease co-causing or as modifying factor. This holds true despite of the somewhat discouraging recent reports, raising doubts as to the significance of any of the candidate genes for schizophrenia [322]. Especially, strongest evidence remains for NRG1 and DTNBP1 [323-325].

The previous sections tried to illustrate different approaches in modeling schizophrenia in rodents. By means of clinical as well as basic research it has become apparent, that schizophrenia is not a monofactorial mental disorder, which affects a single physiologic system or specific brain region with its interconnected functional circuits. Rather it is a complex and polygenetic disease, which might be triggered by different environmental influences. Twin studies have taught us, that heritability has a crucial impact on the evolution of the disease. However, it is not sufficient for triggering schizophrenia since heritability even in monocygotic twins amounts to only $50 \%$ [238, 239]. Logically, the other 50\% stem from environmental influences. Although research in this category is still nascent, we have to put all efforts into creating models that unify multiple causative factors, genetic and environmental, to give rise to the disease.

\section{5. "Future"}

As noted previously, the prevailing notion is that an early developmental deficit alone may not be sufficient to cause the disease. However, an early predisposing factor may increase disease vulnerability. On basis of this 
increased vulnerability - might it be due to genetic predisposition or early brain injury - the illness is believed to be triggered by later life events (e.g. severe stress caused by extreme social isolation, psychotrauma, neurotrauma, drug abuse to just name a few). This interaction of (genetic) vulnerability and additional triggering events has been called the "two-hit" hypothesis of schizophrenia [326, 327]. The basic requirement is believed to be a perhaps minimally dysfunctional genetic background, affecting neuronal development and plasticity, which is fully compensated for in the absence of additional triggers.

There is in fact a growing number of studies combining two or more predisposing and triggering factors. For example, the effects of neonatal ventral hippocampal lesion is modified according to genetic strain [181]. Repeated PCP administration induced a more pronounced hyperlocomotion pattern in rats with neonatal ventral hippocampal lesion [328]. Furthermore, a recent study using different behavioral tasks has demonstrated that heterozygous NRG1 mutant mice are more sensitive to the effects of delta-9tetrahydrocannabinol (THC) administration [329]. Surprisingly, NRG1 mutant mice acutely treated with THC had an enhanced PPI compared with wild type mice. The locomotion suppressant effect of THC was also more pronounced in the NRG1 mutant mice. Despite these rather atypical findings, a rodent model combining two different vulnerability factors seems to be a promising approach for more adequately mimicking the etiology and pathogenesis of schizophrenia. Table 4 gives an overview on the various rodent models discussed in this chapter.

In recent work, the onset of schizophrenia-associated cortical grey matter loss in the parietal lobe [100] was modeled by inducing a small standardized unilateral parietal cold-lesion through the intact skull of 28 day old mice. This lesion, triggering from the outside as yet unknown internal processes that lead to progressive brain atrophy in schizophrenia, led to a similar type of cortical grey matter loss in mice, when applied at the vulnerable age around puberty. Applied at later age, no such changes were observed anymore [330]. Presently, we are combining this model of subtle progressive brain atrophy with genetic and other environmental factors in order to create a "several hit model" of schizophrenia.

The prevailing amount of studies containing multiple vulnerability factors does not intensively focus on assessing the behavioral domains affected in schizophrenia. Moreover, various studies use locomotion as the only behavioral schizophrenia-relevant phenotype. This is not only the case in "multiple-hit" studies. It also holds true for monofactorial designs. Since diagnosing schizophrenia in man relies primarily, if not exclusively, on the behavioral phenotype, an assessment procedure which addresses relevant 


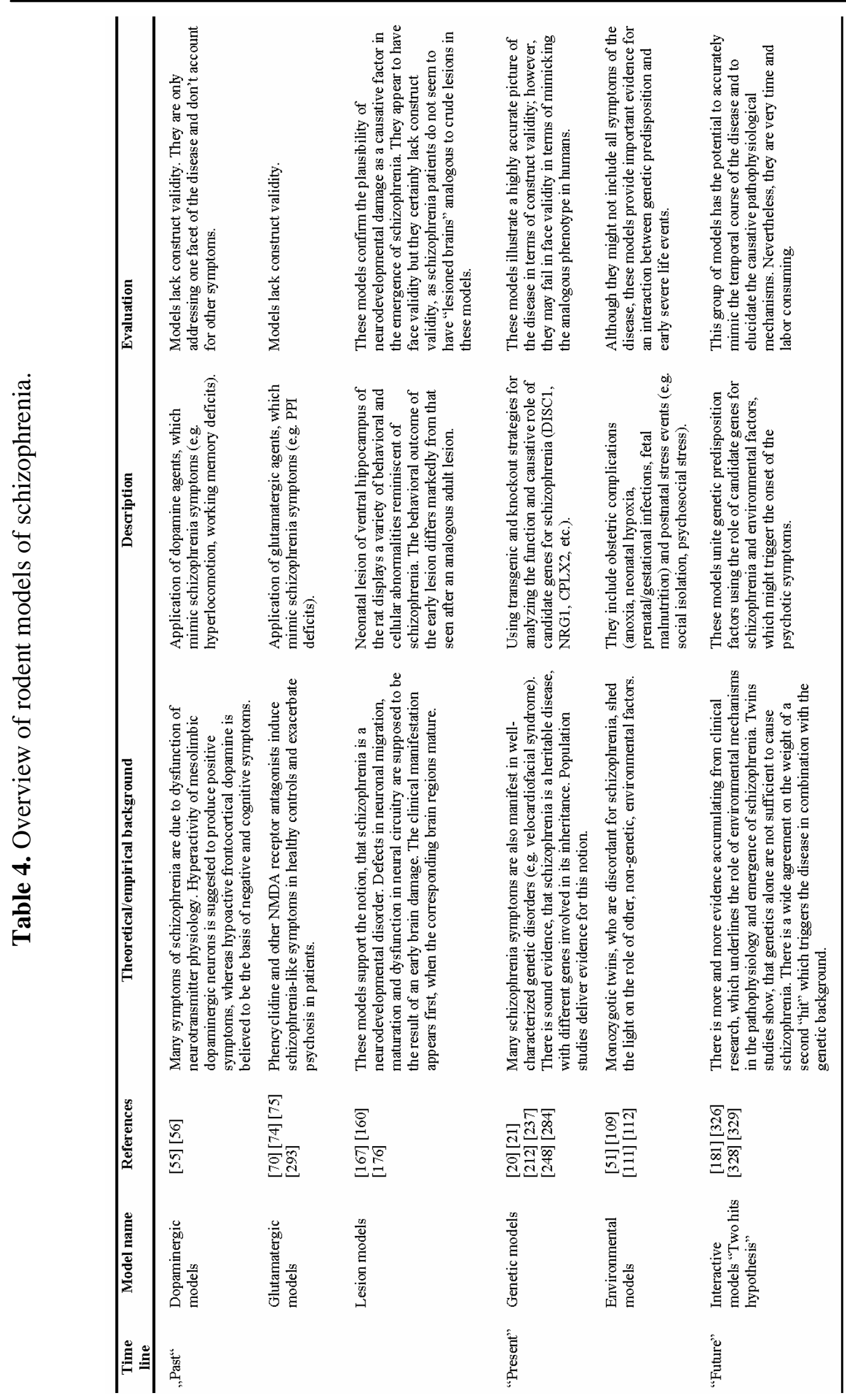


domains also in rodents is indispensable. One potentially promising approach is adapting the diagnostic practice of mental disorders in humans to rodents. Compared with physical diseases, mental disorders lack thus far specific cellular indicators. Thus, the process of diagnosis is heavily based on objective and communicable classification systems. The most famous and widely used diagnosis system for mental health professionals is the Diagnostic and Statistical Manual of Mental Disorders (DSM) of the American Psychiatric Association [4]. The DSM, since 1994 in its $4^{\text {th }}$ revision, is a categorical classification system for various mental disorders. It enables making a diagnosis of the mental functioning of patients by means of using different branches of clinical information. The DSM-IV includes criteria for diagnosing a mental disorder. Symptoms listed there are grouped into clusters. Patients do not have to fulfill all points in all criteria to be given the corresponding diagnosis. Rather, these categories have to be seen as prototypes for the disease and patients showing a high degree of approximation with a given prototype get the corresponding diagnosis. DSM-IV criteria for schizophrenia are summarized in Table 5. For example, according to DSM-IV criteria for schizophrenia, it is necessary to show at least two symptoms of criterion A for the period of one month (or less if successfully treated). Additionally, the patient has to meet criteria B, C and $\mathrm{D}$, while other potential confounding disorders have to be excluded (criteria $\mathrm{E}$ and $\mathrm{F}$ ). However, only one symptom in criterion $\mathrm{A}$ is required, if delusions are bizarre or hallucinations consist of a monologue commenting the behavior of the patient. Despite these objective classification systems, making a diagnosis of schizophrenia in humans is often challenged by various pitfalls. Nevertheless, intensive efforts are made on validating diagnostic methods, e.g. by continuous updates and revisions of DSM and similar classification systems.

Similarly to diagnostic practice in humans, such an objective classification system is desperately needed in rodent models of schizophrenia. As was shown in this chapter, rodents demonstrate various behavioral features analogous to humans. Thus, the formulation of objective diagnostic criteria for "rodent schizophrenia" seems to have a factual basis.

In the following section, we offer a preliminary step towards defining communicable criteria for the diagnosis of schizophrenia in rodent models. As stated previously, the diagnostic process should account for the mainly behavioral phenotype of the disease. For the sake of simplicity, we will call these criteria "rodent DSM criteria", pointing to our intention of having analogous conditions to human diagnostic standards. The key principle which is implicated in these criteria is that the diagnosis is made on the basis of constellation of specific symptoms, rather than of the presence of single 
features of the disease. In analogy with the human diagnostic manual, a rodent diagnostic system has to include the combination of sufficient diseaserelated symptoms in different behavioral domains. It has to be stated at this point, that the formulation of such diagnostic criteria is dependent on current knowledge of disease symptomatology and underlying mechanisms. The state of knowledge is target of steadily growing developments and achievements in science. Thus, the criteria we propose here for rodents will be as much subject to future revisions as the DSM criteria in humans.

Table 5. Diagnostic criteria for schizophrenia according to DSM-IV (AmericanPsychiatric-Association 2000).

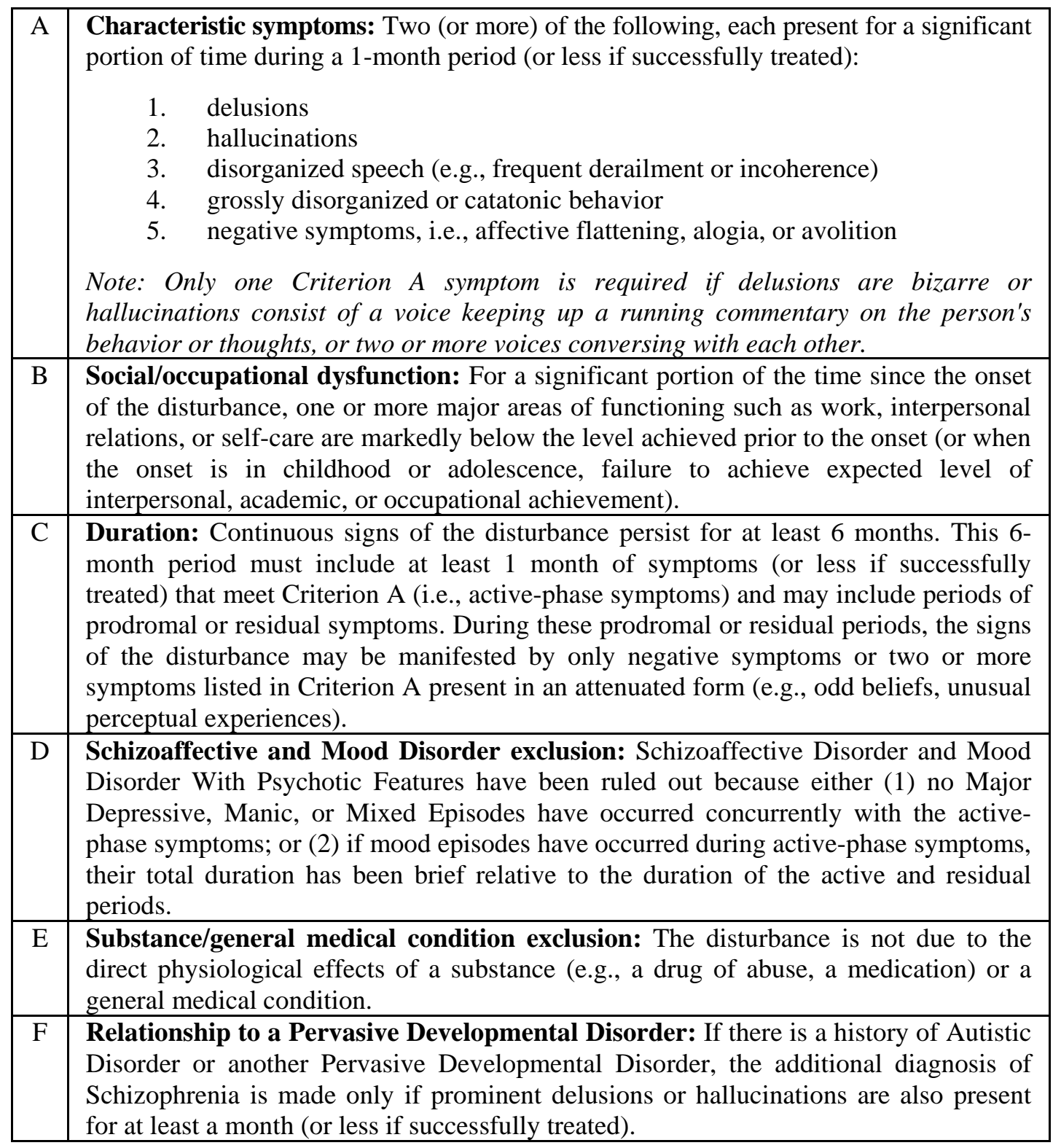




\subsection{DSM criteria for rodent schizophrenia}

As discussed at the beginning of this chapter, animal models of psychiatric disorders have to meet some validity requirements to be regarded as scientifically beneficial. Consequently, we are convinced that good construct validity of a potential model should be regarded as the first criterion, delivering category $\mathbf{A}$ which has to be met in a diagnostic system for rodent schizophrenia (Table 6). Thus, a potential experimental intervention should meet this key prerequisite. For example, animal models based on the "two-hit" hypothesis implicating combinations of candidate genes and environmental factors fulfill this requirement at best. However, the assessment of construct validity heavily depends on the growing understanding of the disease. Thus, this point has to be evaluated according to the contemporary disease knowledge.

Furthermore, we suggest to group different schizophrenia symptoms into classes according to domains (compare also Table 1). This approach addresses the face validity issue and puts positive and negative symptoms together in category B. As mentioned previously, there are almost no reliable behavioral features in rodents, which are homologous to human positive symptoms (e.g. hallucinations and delusions). Nevertheless, it is widely believed, that hyperlocomotion/hyperactivity and stereotypy are to some extent related to positive symptomatology. One important feature of negative symptoms is social withdrawal, which can be assessed in rodents. Similarly, some aspects of affective loss/anhedonia can be modeled. Consequently, a potential rodent model of schizophrenia should fulfill at least one of the characteristic features listed in category B.

As cognitive symptoms are believed to be a core feature of the disease [2], we suggest to include them in rodent DSM, even though very surprisingly they were not addressed (forgotten?) in the current version ( $4^{\text {th }}$ ed.) of human DSM. Cognitive functions have been intensively studied in rodents, with various well-established and reliable tests available. Based on that, we include readouts for working memory, attention, executive functions and sensorimotor gating in category C. A potential "schizophrenic" rodent should show deficits in at least two characteristic features listed in this category. It has to be noted here, that rodents showing a global cognitive deficit with very low learning ability have to be excluded. Schizophrenia is not comparable with mental retardation or debility.

Concerning predictive validity, we suggest to include the reversal of symptoms by antipsychotics as part of an optional, supporting category $\mathbf{D}$. We feel that it should be optional since not all schizophrenic patients benefit from antipsychotic medication [68]. However, a putative rodent model showing reversal of symptoms after antipsychotic medication should be regarded as most reliable. 
Table 6. Rodent DSM-like criteria for modelling schizophrenia.

\begin{tabular}{|c|c|c|c|c|}
\hline $\begin{array}{l}\text { Validity } \\
\text { aspects }\end{array}$ & Category & Domain & \multicolumn{2}{|c|}{ Characteristic features } \\
\hline $\begin{array}{l}\text { Construct } \\
\text { validity }\end{array}$ & A & & \multicolumn{2}{|c|}{$\begin{array}{l}\text { The means of modeling applied in rodents should stay in } \\
\text { conceptual correspondence with human disease }\end{array}$} \\
\hline \multirow{8}{*}{$\begin{array}{l}\text { Face } \\
\text { validity }\end{array}$} & B & $\begin{array}{l}\text { Positive } \\
\text { symptoms }\end{array}$ & $\begin{array}{l}\text { Psychomotor } \\
\text { agitation }\end{array}$ & $\begin{array}{l}\text { Spontaneous hyperlocomotion } \\
\text { Increase in PCP- and MK801-induced } \\
\text { hyperlocomotion } \\
\text { Increase in amphetamine induced } \\
\text { hyperlocomotion } \\
\text { Increase in stress induced } \\
\text { hyperlocomotion }\end{array}$ \\
\hline & & & Stereotypy & $\begin{array}{l}\text { Circling movements } \\
\text { Repetitive behaviors }\end{array}$ \\
\hline & & $\begin{array}{l}\text { Negative } \\
\text { symptoms }\end{array}$ & Anhedonia & Decrease in sucrose preference \\
\hline & & & $\begin{array}{l}\text { Social } \\
\text { withdrawal }\end{array}$ & $\begin{array}{l}\text { Decreased preference to social novelty } \\
\text { Decreased social interaction } \\
\text { Decreased or disturbed nesting in the } \\
\text { home cage } \\
\text { Decreased huddling in the home cage }\end{array}$ \\
\hline & \multirow[t]{4}{*}{ C } & $\begin{array}{l}\text { Cognitive } \\
\text { symptoms }\end{array}$ & $\begin{array}{l}\text { Working } \\
\text { memory deficits }\end{array}$ & $\begin{array}{l}\text { Impaired performance in T-maze } \\
\text { Impaired performance in 8-arm maze }\end{array}$ \\
\hline & & & Deficit of attentior & $\begin{array}{l}\text { Impaired latent inhibition } \\
\text { Decreased sustained attention in } \\
\text { 5CSRTT } \\
\text { Increased distractibility in 5CSRTT }\end{array}$ \\
\hline & & & $\begin{array}{l}\text { Executive } \\
\text { function deficits }\end{array}$ & $\begin{array}{l}\text { Increased reaction time in 5CSRTT } \\
\text { Increased premature responses in } \\
\text { 5CSRTT } \\
\text { Decreased set-shifting ability }\end{array}$ \\
\hline & & & $\begin{array}{l}\text { Deficit of } \\
\text { sensorimotor } \\
\text { gating }\end{array}$ & $\begin{array}{l}\text { Diminished PPI } \\
\text { Deficit of startle habituation }\end{array}$ \\
\hline
\end{tabular}

Note: A global cognitive deficient phenotype with very low learning ability has to be excluded.

\begin{tabular}{|c|c|c|}
\hline & & Optional, supporting categories \\
\hline $\begin{array}{ll}\text { Predictive } \mathbf{D} \\
\text { validity }\end{array}$ & & $\begin{array}{l}\text { Improvement of some deficits from categories } \mathbf{B} \text { and } \mathbf{C} \\
\text { after treatment with known antipsychotic drugs }\end{array}$ \\
\hline $\mathbf{E}$ & $\begin{array}{l}\text { Non- } \\
\text { behavioral } \\
\text { traits }\end{array}$ & $\begin{array}{l}\text { Findings in neuroimaging, neuropathology, pathophysiology } \\
\text { and gene expression comparable to schizophrenia patients }\end{array}$ \\
\hline
\end{tabular}


In contrast to diagnostic criteria in humans, we propose several additional invasive assessment methods for detecting morphological, cellular and physiological abnormalities shown in schizophrenic patients. Even though these findings are not consistent in all affected human individuals, they might ultimately help to formulate and detect subtypes of the disease in humans as well as rodents. These diagnostic means are considered in the second optional, supporting category $\mathbf{E}$.

The here proposed criteria should be regarded as an attempt to guide the modeling of such complex biological disease phenomena as schizophrenia. The steadily growing advances in translational neuroscience and biological psychiatry will hopefully help to revise and modify the above mentioned categories for classification of rodent schizophrenia. Rigorous scientific efforts are desperately required in order to fulfill the substantial need for valid animal models of schizophrenia. We are convinced that further developments in this field will lead to deeper understanding of the underlying disease mechanisms as well as to advances in therapeutic and preventive approaches.

\section{References}

1. Wong, A.H. and Van Tol, H.H. 2003, Neurosci Biobehav Rev, 27, 269.

2. Elvevag, B. and Goldberg, T.E. 2000, Crit Rev Neurobiol, 14, 1.

3. Lewis, D.A. and Lieberman, J.A. 2000, Neuron, 28, 325.

4. American-Psychiatric-Association 2000, Diagnostic and Statistical Manual of Mental Disorders. 4th ed. American Psychiatric Association, Washington, DC.

5. Adityanjee, Aderibigbe, Y.A., Theodoridis, D. and Vieweg, V.R. 1999, Psychiatry Clin Neurosci, 53, 437.

6. Ross, C.A., Margolis, R.L., Reading, S.A., Pletnikov, M. and Coyle, J.T. 2006, Neuron, 52, 139.

7. Ellenbroek, B.A. and Cools, A.R. 1990, Behav Pharmacol, 1, 469.

8. Kornetsky, C. and Markowitz, R. 1975, Model Systems in Biological Psychiatry. Ingle, D.J. and Shein, H.M. (Ed.). MIT Press, Cambridge, Massachusetts, 26.

9. McKinney, W.T. and Moran, E.C. 1981, Am J Psychiatry, 138, 478.

10. Marcotte, E.R., Pearson, D.M. and Srivastava, L.K. 2001, J Psychiatry Neurosci, 26, 395.

11. Lipska, B.K. and Weinberger, D.R. 2000, Neuropsychopharmacology, 23, 223.

12. McKinney, W.T., Jr. and Bunney, W.E., Jr. 1969, Arch Gen Psychiatry, 21, 240.

13. Willner, P. 1986, Prog Neuropsychopharmacol Biol Psychiatry, 10, 677.

14. van der Staay, F.J. 2006, Brain Res Rev, 52, 131.

15. Matthysse, S. 1986, Prog Brain Res, 65, 259.

16. Kornetsky, C. and Markowitz, R. 1978, Psychopharmacology: A Generation of Progress. Lipton, M., DiMascio, A. and Killam, K. (Ed.). Raven Press, New York, 583. 
17. Matthysse, S. and Haber, S. 1975, Model Systems in Biological Psychiatry. Ingle, D.J. and Shein, H.M. (Ed.). MIT Press, Cambridge, Massachusetts, 4.

18. Willner, P. 1984, Psychopharmacology (Berl), 83, 1.

19. Powell, C.M. and Miyakawa, T. 2006, Biol Psychiatry, 59, 1198.

20. Gogos, J.A., Morgan, M., Luine, V., Santha, M., Ogawa, S., Pfaff, D. and Karayiorgou, M. 1998, Proc Natl Acad Sci U S A, 95, 9991.

21. Clapcote, S.J., Lipina, T.V., Millar, J.K., Mackie, S., Christie, S., Ogawa, F., Lerch, J.P., Trimble, K., Uchiyama, M., Sakuraba, Y. et al. 2007, Neuron, 54, 387.

22. Stefansson, H., Sigurdsson, E., Steinthorsdottir, V., Bjornsdottir, S., Sigmundsson, T., Ghosh, S., Brynjolfsson, J., Gunnarsdottir, S., Ivarsson, O., Chou, T.T. et al. 2002, Am J Hum Genet, 71, 877.

23. Borison, R.L., Havdala, H.S. and Diamond, B.I. 1977, Life Sci, 21, 117.

24. Lipska, B.K., Jaskiw, G.E. and Weinberger, D.R. 1991, Pharmacol Biochem Behav, 40, 169.

25. Ridley, R.M. 1994, Prog Neurobiol, 44, 221.

26. Iversen, S.D. and Koob, G.F. 1977, Adv Biochem Psychopharmacol, 16, 209.

27. Vickery, S.S. and Mason, G.J. 2005, Applied Animal Behaviour Science, 91, 247.

28. Garner, J.P. and Mason, G.J. 2002, Behav Brain Res, 136, 83.

29. Ellenbroek, B.A. and Cools, A.R. 2002, Behav Genet, 32, 349.

30. Bellack, A.S., Morrison, R.L., Wixted, J.T. and Mueser, K.T. 1990, Br J Psychiatry, 156, 809.

31. Couture, S.M., Penn, D.L. and Roberts, D.L. 2006, Schizophr Bull, 32 Suppl 1, S44.

32. Portfors, C.V. 2007, J Am Assoc Lab Anim Sci, 46, 28.

33. Jamain, S., Radyushkin, K., Hammerschmidt, K., Granon, S., Boretius, S., Varoqueaux, F., Ramanantsoa, N., Gallego, J., Ronnenberg, A., Winter, D. et al. 2008, Proc Natl Acad Sci U S A, 105, 1710.

34. Ellenbroek, B.A. and Cools, A.R. 2000, Behav Pharmacol, 11, 223.

35. Braff, D.L., Geyer, M.A. and Swerdlow, N.R. 2001, Psychopharmacology (Berl), 156, 234.

36. Braff, D.L. and Light, G.A. 2004, Psychopharmacology (Berl), 174, 75.

37. Swerdlow, N.R., Braff, D.L. and Geyer, M.A. 2000, Behav Pharmacol, 11, 185.

38. Geyer, M.A., Krebs-Thomson, K., Braff, D.L. and Swerdlow, N.R. 2001, Psychopharmacology (Berl), 156, 117.

39. Lubow, R.E. 2005, Schizophr Bull, 31, 139.

40. Swerdlow, N.R., Braff, D.L., Hartston, H., Perry, W. and Geyer, M.A. 1996, Schizophr Res, 20, 91.

41. Weiner, I., Lubow, R.E. and Feldon, J. 1988, Pharmacol Biochem Behav, 30, 871.

42. Moser, P.C., Hitchcock, J.M., Lister, S. and Moran, P.M. 2000, Brain Res Brain Res Rev, 33, 275.

43. Weiner, I., Shadach, E., Tarrasch, R., Kidron, R. and Feldon, J. 1996, Biol Psychiatry, 40, 834. 
44. Barch, D.M. 2005, Annu Rev Clin Psychol, 1, 321.

45. Perlstein, W.M., Carter, C.S., Noll, D.C. and Cohen, J.D. 2001, Am J Psychiatry, 158, 1105.

46. Barch, D.M. and Csernansky, J.G. 2007, Am J Psychiatry, 164, 1090.

47. Barch, D.M., Sheline, Y.I., Csernansky, J.G. and Snyder, A.Z. 2003, Biol Psychiatry, 53, 376.

48. Baddeley, A. 1996, Proc Natl Acad Sci U S A, 93, 13468.

49. Dudchenko, P.A. 2004, Neurosci Biobehav Rev, 28, 699.

50. Pontecorvo, M.J., Sahgal, A. and Steckler, T. 1996, Brain Res Cogn Brain Res, 3, 205.

51. Weiss, I.C. and Feldon, J. 2001, Psychopharmacology (Berl), 156, 305.

52. Carlsson, A. 1977, Psychol Med, 7, 583.

53. van Kammen, D.P. 1979, Psychoneuroendocrinology, 4, 37.

54. Davis, J.M. 1974, J Psychiatr Res, 11, 25.

55. Davis, K.L., Kahn, R.S., Ko, G. and Davidson, M. 1991, Am J Psychiatry, 148, 1474.

56. Abi-Dargham, A. 2004, Int J Neuropsychopharmacol, 7 Suppl 1, S1.

57. Griffith, J.J., Oates, J. and Cavanaugh, J. 1968, J. Am. Med. Ass., 205.

58. Connell, P.H. 1958, Amphetamine Psychosis. Oxford University Press Oxford.

59. Hutchison, K.E. and Swift, R. 1999, Psychopharmacology (Berl), 143, 394.

60. Swerdlow, N.R., Braff, D.L., Taaid, N. and Geyer, M.A. 1994, Arch Gen Psychiatry, 51, 139.

61. Parwani, A., Duncan, E.J., Bartlett, E., Madonick, S.H., Efferen, T.R., Rajan, R., Sanfilipo, M., Chappell, P.B., Chakravorty, S., Gonzenbach, S. et al. 2000, Biol Psychiatry, 47, 662.

62. Braff, D.L. and Geyer, M.A. 1990, Arch Gen Psychiatry, 47, 181.

63. Hamm, A.O., Weike, A.I. and Schupp, H.T. 2001, Psychopharmacology (Berl), 156, 259.

64. Swerdlow, N.R., Talledo, J., Sutherland, A.N., Nagy, D. and Shoemaker, J.M. 2006, Neuropsychopharmacology, 31, 2011.

65. Klaning, U., Mortensen, P.B. and Kyvik, K.O. 1996, Br J Psychiatry, 168, 688.

66. Angrist, B., Sathananthan, G., Wilk, S. and Gershon, S. 1974, J Psychiatr Res, $11,13$.

67. Mackeprang, T., Kristiansen, K.T. and Glenthoj, B.Y. 2002, Biol Psychiatry, 52, 863.

68. Conley, R.R. and Kelly, D.L. 2001, Biol Psychiatry, 50, 898.

69. Krystal, J.H., Karper, L.P., Seibyl, J.P., Freeman, G.K., Delaney, R., Bremner, J.D., Heninger, G.R., Bowers, M.B., Jr. and Charney, D.S. 1994, Arch Gen Psychiatry, 51, 199.

70. Meyer, J.S., Greifenstein, F. and Devault, M. 1959, Journal of Nervous and Mental Disease, 129, 54.

71. Kim, J.S., Kornhuber, H.H., Schmid-Burgk, W. and Holzmuller, B. 1980, Neurosci Lett, 20, 379.

72. Bakshi, V.P., Swerdlow, N.R. and Geyer, M.A. 1994, J Pharmacol Exp Ther, 271, 787. 
73. Bakshi, V.P. and Geyer, M.A. 1995, Psychopharmacology (Berl), 122, 198.

74. Moghaddam, B. and Jackson, M.E. 2003, Ann N Y Acad Sci, 1003, 131.

75. Jentsch, J.D. and Roth, R.H. 1999, Neuropsychopharmacology, 20, 201.

76. Hauber, W. and Schmidt, W.J. 1989, J Neural Transm Gen Sect, 78, 29.

77. Mansbach, R.S. and Geyer, M.A. 1989, Neuropsychopharmacology, 2, 299.

78. Steinpreis, R.E., Sokolowski, J.D., Papanikolaou, A. and Salamone, J.D. 1994, Pharmacol Biochem Behav, 47, 579.

79. Duncan, E.J., Madonick, S.H., Parwani, A., Angrist, B., Rajan, R., Chakravorty, S., Efferen, T.R., Szilagyi, S., Stephanides, M., Chappell, P.B. et al. 2001, Neuropsychopharmacology, 25, 72.

80. Carlsson, M. and Carlsson, A. 1990, Trends Neurosci, 13, 272.

81. McCarley, R.W., Wible, C.G., Frumin, M., Hirayasu, Y., Levitt, J.J., Fischer, I.A. and Shenton, M.E. 1999, Biol Psychiatry, 45, 1099.

82. Staal, W.G., Hulshoff Pol, H.E., Schnack, H.G., Hoogendoorn, M.L., Jellema, K. and Kahn, R.S. 2000, Am J Psychiatry, 157, 416.

83. Hughes, C., Kumari, V., Das, M., Zachariah, E., Ettinger, U., Sumich, A. and Sharma, T. 2005, Acta Psychiatr Scand, 111, 185.

84. Barrantes-Vidal, N., Aguilera, M., Campanera, S., Fatjo-Vilas, M., Guitart, M., Miret, S., Valero, S. and Fananas, L. 2007, Schizophr Res, 95, 70.

85. Weinberger, D.R., Berman, K.F. and Zec, R.F. 1986, Arch Gen Psychiatry, 43, 114.

86. Suddath, R.L., Christison, G.W., Torrey, E.F., Casanova, M.F. and Weinberger, D.R. 1990, N Engl J Med, 322, 789.

87. Cannon, T.D., van Erp, T.G., Huttunen, M., Lonnqvist, J., Salonen, O., Valanne, L., Poutanen, V.P., Standertskjold-Nordenstam, C.G., Gur, R.E. and Yan, M. 1998, Arch Gen Psychiatry, 55, 1084.

88. Goldstein, J.M., Goodman, J.M., Seidman, L.J., Kennedy, D.N., Makris, N., Lee, H., Tourville, J., Caviness, V.S., Jr., Faraone, S.V. and Tsuang, M.T. 1999, Arch Gen Psychiatry, 56, 537.

89. Hirayasu, Y., Tanaka, S., Shenton, M.E., Salisbury, D.F., DeSantis, M.A., Levitt, J.J., Wible, C., Yurgelun-Todd, D., Kikinis, R., Jolesz, F.A. et al. 2001, Cereb Cortex, 11, 374.

90. Wible, C.G., Shenton, M.E., Hokama, H., Kikinis, R., Jolesz, F.A., Metcalf, D. and McCarley, R.W. 1995, Arch Gen Psychiatry, 52, 279.

91. Weinberger, D.R. 1991, Biol Psychiatry, 29, 509.

92. Heckers, S., Rauch, S.L., Goff, D., Savage, C.R., Schacter, D.L., Fischman, A.J. and Alpert, N.M. 1998, Nat Neurosci, 1, 318.

93. Weinberger, D.R., Berman, K.F. and Illowsky, B.P. 1988, Arch Gen Psychiatry, 45, 609.

94. Pycock, C.J., Kerwin, R.W. and Carter, C.J. 1980, Nature, 286, 74.

95. Falkai, P. and Bogerts, B. 1986, Eur Arch Psychiatry Neurol Sci, 236, 154.

96. Harrison, P.J. 1999, Brain, 122, 593.

97. Church, S.M., Cotter, D., Bramon, E. and Murray, R.M. 2002, J Neural Transm Suppl, 129.

98. Lieberman, J.A. 1999, Biol Psychiatry, 46, 729. 
99. Ojeda, N., Sanchez, P., Elizagarate, E., Yoller, A.B., Ezcurra, J., Ramirez, I. and Ballesteros, J. 2007, Actas Esp Psiquiatr, 35, 263.

100. Thompson, P.M., Vidal, C., Giedd, J.N., Gochman, P., Blumenthal, J., Nicolson, R., Toga, A.W. and Rapoport, J.L. 2001, Proc Natl Acad Sci U S A, 98, 11650.

101.Hoff, A.L., Sakuma, M., Razi, K., Heydebrand, G., Csernansky, J.G. and DeLisi, L.E. 2000, Am J Psychiatry, 157, 1824.

102. Bogerts, B. 1993, Schizophr Bull, 19, 431.

103. Croisier, E. and Graeber, M.B. 2006, Acta Neuropathol, 112, 517.

104. Jarskog, L.F., Gilmore, J.H., Selinger, E.S. and Lieberman, J.A. 2000, Biol Psychiatry, 48, 641.

105. Jarskog, L.F. 2006, Curr Opin Psychiatry, 19, 307.

106. Purohit, D.P., Perl, D.P., Haroutunian, V., Powchik, P., Davidson, M. and Davis, K.L. 1998, Arch Gen Psychiatry, 55, 205.

107. Mockler, D., Riordan, J. and Sharma, T. 1997, Schizophr Res, 26, 1.

108. Lipska, B.K. 2004, J Psychiatry Neurosci, 29, 282.

109. Harms, L.R., Eyles, D.W., McGrath, J.J., Mackay-Sim, A. and Burne, T.H. 2008, Behav Brain Res, 187, 343.

110. Cintra, L., Granados, L., Aguilar, A., Kemper, T., DeBassio, W., Galler, J., Morgane, P., Duran, P. and Diaz-Cintra, S. 1997, Hippocampus, 7, 184.

111. Cotter, D., Takei, N., Farrell, M., Sham, P., Quinn, P., Larkin, C., Oxford, J., Murray, R.M. and O'Callaghan, E. 1995, Schizophr Res, 16, 233.

112. Brake, W.G., Boksa, P. and Gratton, A. 1997, Psychopharmacology (Berl), 133, 389.

113. Black, M.D., Selk, D.E., Hitchcock, J.M., Wettstein, J.G. and Sorensen, S.M. 1999, Neuropharmacology, 38, 1299.

114. Liu, D., Diorio, J., Tannenbaum, B., Caldji, C., Francis, D., Freedman, A., Sharma, S., Pearson, D., Plotsky, P.M. and Meaney, M.J. 1997, Science, 277, 1659 .

115. Coyle, J.T. and Schwarcz, R. 1983, Methods in Chemical Neuroanatomy. Björklund, A. and Hökfelt, T. (Ed.). Elsevier, New York, 508.

116. Breese, G.R. and Traylor, T.D. 1970, J Pharmacol Exp Ther, 174, 413.

117. Breese, G.R., Knapp, D.J., Criswell, H.E., Moy, S.S., Papadeas, S.T. and Blake, B.L. 2005, Brain Res Brain Res Rev, 48, 57.

118. Lipska, B.K., Halim, N.D., Segal, P.N. and Weinberger, D.R. 2002, J Neurosci, 22, 2835.

119. Suzuki, M., Nohara, S., Hagino, H., Takahashi, T., Kawasaki, Y., Yamashita, I., Watanabe, N., Seto, H. and Kurachi, M. 2005, Psychiatry Res, 140, 157.

120. Yoon, T., Okada, J., Jung, M.W. and Kim, J.J. 2008, Learn Mem, 15, 97.

121. Braun, A.R., Jaskiw, G.E., Vladar, K., Sexton, R.H., Kolachana, B.S. and Weinberger, D.R. 1993, Pharmacol Biochem Behav, 46, 51.

122. Lipska, B.K., Jaskiw, G.E., Braun, A.R. and Weinberger, D.R. 1995, Biol Psychiatry, 38, 255.

123. Swerdlow, N.R., Lipska, B.K., Weinberger, D.R., Braff, D.L., Jaskiw, G.E. and Geyer, M.A. 1995, Psychopharmacology (Berl), 122, 27.

124. Jaskiw, G.E., Karoum, F.K. and Weinberger, D.R. 1990, Brain Res, 534, 321. 
125. Miner, L.A., Ostrander, M. and Sarter, M. 1997, J Psychopharmacol, 11, 169.

126. Joel, D., Weiner, I. and Feldon, J. 1997, Behav Brain Res, 85, 187.

127. Lacroix, L., Spinelli, S., White, W. and Feldon, J. 2000, Neuroscience, 97, 459.

128. Jaskiw, G.E., Karoum, F., Freed, W.J., Phillips, I., Kleinman, J.E. and Weinberger, D.R. 1990, Brain Res, 534, 263.

129. Harden, D.G., King, D., Finlay, J.M. and Grace, A.A. 1998, Brain Res, 794, 96.

130. King, D., Zigmond, M.J. and Finlay, J.M. 1997, Neuroscience, 77, 141.

131. Wise, R.A. 2000, Prog Brain Res, 126, 255.

132. Bordi, F. and Meller, E. 1989, Brain Res, 504, 276.

133. Delfs, J.M. and Kelley, A.E. 1990, Neuroscience, 39, 59.

134. LaHoste, G.J. and Marshall, J.F. 1990, Behav Brain Res, 38, 233.

135. Subramaniam, S., Lucki, I. and McGonigle, P. 1992, Brain Res, 571, 313.

136. Herrero, M.T., Barcia, C. and Navarro, J.M. 2002, Childs Nerv Syst, 18, 386.

137. Swerdlow, N.R., Geyer, M.A. and Braff, D.L. 2001, Psychopharmacology (Berl), 156, 194.

138. Hazlett, E.A., Buchsbaum, M.S., Kemether, E., Bloom, R., Platholi, J., Brickman, A.M., Shihabuddin, L., Tang, C. and Byne, W. 2004, Am J Psychiatry, 161, 305.

139. Buchsbaum, M.S., Someya, T., Teng, C.Y., Abel, L., Chin, S., Najafi, A., Haier, R.J., Wu, J. and Bunney, W.E., Jr. 1996, Am J Psychiatry, 153, 191.

140. Andrews, J., Wang, L., Csernansky, J.G., Gado, M.H. and Barch, D.M. 2006, Am J Psychiatry, 163, 463.

141. Andreasen, N.C., Arndt, S., Swayze, V., 2nd, Cizadlo, T., Flaum, M., O'Leary, D., Ehrhardt, J.C. and Yuh, W.T. 1994, Science, 266, 294.

142. Arciniegas, D., Rojas, D.C., Teale, P., Sheeder, J., Sandberg, E. and Reite, M. 1999, Biol Psychiatry, 45, 1329.

143. Sutherland, R.J. 1982, Neurosci Biobehav Rev, 6, 1.

144. Sandyk, R. 1991, Int J Neurosci, 61, 189.

145. Sandyk, R. 1992, Int J Neurosci, 67, 19.

146. Ellison, G. 1994, Brain Res Brain Res Rev, 19, 223.

147. Lecourtier, L. and Kelly, P.H. 2005, Neuropsychopharmacology, 30, 484.

148. Harms, M.P., Wang, L., Mamah, D., Barch, D.M., Thompson, P.A. and Csernansky, J.G. 2007, J Neurosci, 27, 13835.

149. Mathalon, D.H., Heinks, T. and Ford, J.M. 2004, Am J Psychiatry, 161, 872.

150. Schmahmann, J.D. 2003, Stroke, 34, 2264.

151. Bardgett, M.E., Jackson, J.L., Taylor, G.T. and Csernansky, J.G. 1995, Behav Brain Res, 70, 153.

152. Bardgett, M.E., Jacobs, P.S., Jackson, J.L. and Csernansky, J.G. 1997, Behav Brain Res, 84, 47.

153. Csernansky, J.G., Csernansky, C.A., Kogelman, L., Montgomery, E.M. and Bardgett, M.E. 1998, Biol Psychiatry, 44, 1143.

154. Goldman, M.B. and Mitchell, C.P. 2004, Schizophr Bull, 30, 367.

155. Tierney, P.L., Degenetais, E., Thierry, A.M., Glowinski, J. and Gioanni, Y. 2004, Eur J Neurosci, 20, 514.

156. Thierry, A.M., Gioanni, Y., Degenetais, E. and Glowinski, J. 2000, Hippocampus, 10, 411. 
157. Kelley, A.E. and Domesick, V.B. 1982, Neuroscience, 7, 2321.

158. Jay, T.M., Glowinski, J. and Thierry, A.M. 1989, Brain Res, 505, 337.

159. Lipska, B.K., Jaskiw, G.E., Karoum, F., Phillips, I., Kleinman, J.E. and Weinberger, D.R. 1991, Pharmacol Biochem Behav, 40, 181.

160. Lipska, B.K., Jaskiw, G.E., Chrapusta, S., Karoum, F. and Weinberger, D.R. 1992, Brain Res, 585, 1.

161. Lanier, L.P. and Isaacson, R.L. 1975, Behav Biol, 13, 59.

162. Kelley, A.E., Domesick, V.B. and Nauta, W.J. 1982, Neuroscience, 7, 615.

163. Swanson, L.W. and Cowan, W.M. 1977, J Comp Neurol, 172, 49.

164. Weinberger, D.R. 1987, Arch Gen Psychiatry, 44, 660.

165. Kennard, M. 1936, Am J Physiol, 115, 138.

166. Lipska, B.K., Jaskiw, G.E. and Weinberger, D.R. 1993, Neuropsychopharmacology, 9, 67.

167. Lipska, B.K. and Weinberger, D.R. 1993, Brain Res Dev Brain Res, 75, 213.

168. Lipska, B.K. and Weinberger, D.R. 1994, Neuropsychopharmacology, 10, 199.

169. Sams-Dodd, F., Lipska, B.K. and Weinberger, D.R. 1997, Psychopharmacology (Berl), 132, 303.

170. Lipska, B.K., Chrapusta, S.J., Egan, M.F. and Weinberger, D.R. 1995, Synapse, 20, 125.

171. Chambers, R.A. and Self, D.W. 2002, Neuropsychopharmacology, 27, 889.

172. Le Pen, G., Gaudet, L., Mortas, P., Mory, R. and Moreau, J.L. 2002, Psychopharmacology (Berl), 161, 434.

173. Becker, A., Grecksch, G., Bernstein, H.G., Hollt, V. and Bogerts, B. 1999, Psychopharmacology (Berl), 144, 333.

174. Becker, A. and Grecksch, G. 2003, Pharmacol Biochem Behav, 76, 1.

175. Becker, A. and Grecksch, G. 2000, Behav Brain Res, 109, 137.

176. Chambers, R.A., Moore, J., McEvoy, J.P. and Levin, E.D. 1996, Neuropsychopharmacology, 15, 587.

177. Lipska, B.K., Aultman, J.M., Verma, A., Weinberger, D.R. and Moghaddam, B. 2002, Neuropsychopharmacology, 27, 47.

178. Grecksch, G., Bernstein, H.G., Becker, A., Hollt, V. and Bogerts, B. 1999, Neuropsychopharmacology, 20, 525.

179. Le Pen, G. and Moreau, J.L. 2002, Neuropsychopharmacology, 27, 1.

180. Lipska, B.K., Swerdlow, N.R., Geyer, M.A., Jaskiw, G.E., Braff, D.L. and Weinberger, D.R. 1995, Psychopharmacology (Berl), 122, 35.

181. Lipska, B.K. and Weinberger, D.R. 1995, Proc Natl Acad Sci U S A, 92, 8906.

182. Lipska, B.K. and Weinberger, D.R. 1994, Brain Res Dev Brain Res, 78, 253.

183. Goto, Y. and O'Donnell, P. 2004, Biol Psychiatry, 55, 172.

184. Akbarian, S. and Huang, H.S. 2006, Brain Res Rev, 52, 293.

185. Angelucci, F., Brene, S. and Mathe, A.A. 2005, Mol Psychiatry, 10, 345.

186. Khaing, Z.Z., Weickert, C.S., Weinberger, D.R. and Lipska, B.K. 2000, Eur J Neurosci, 12, 4424.

187. Lipska, B.K. and Weinberger, D.R. 2002, Neurotox Res, 4, 469.

188. Jablensky, A. 1997, Schizophr Res, 28, 111. 
189. Owen, M.J., O'Donovan, M.C. and Gottesman, I.I. 2002, Psychiatric Genetics \& Genomics. McGuffin, P., Owen, M.J. and Gottesman, I.I. (Ed.). Oxford University Press, Oxford, 247.

190. Gottesman, I.I. 1991, Schizophrenia Genesis: The Origins of Madness. Freeman, New York.

191. Jablensky, A. and Sartorius, N. 1988, Acta Psychiatr Scand Suppl, 344, 65.

192. Bromet, E.J. and Fennig, S. 1999, Biol Psychiatry, 46, 871.

193. Waddington, J.L., Corvin, A.P., Donohoe, G., O'Tuathaigh, C.M., Mitchell, K.J. and Gill, M. 2007, Psychiatr Clin North Am, 30, 365.

194. Badner, J.A. and Gershon, E.S. 2002, Mol Psychiatry, 7, 405.

195. Lewis, C.M., Levinson, D.F., Wise, L.H., DeLisi, L.E., Straub, R.E., Hovatta, I., Williams, N.M., Schwab, S.G., Pulver, A.E., Faraone, S.V. et al. 2003, Am J Hum Genet, 73, 34.

196. Tecott, L.H. 2003, Am J Psychiatry, 160, 646.

197. Pennacchio, L.A. 2003, Mamm Genome, 14, 429.

198. Ryding, A.D., Sharp, M.G. and Mullins, J.J. 2001, J Endocrinol, 171, 1.

199. Bucan, M. and Abel, T. 2002, Nat Rev Genet, 3, 114.

200. Muller, M.B. and Keck, M.E. 2002, J Psychiatr Res, 36, 53.

201. Bult, C.J., Eppig, J.T., Kadin, J.A., Richardson, J.E. and Blake, J.A. 2008, Nucleic Acids Res, 36, D724.

202. Waterston, R.H. and Lindblad-Toh, K. and Birney, E. and Rogers, J. and Abril, J.F. and Agarwal, P. and Agarwala, R. and Ainscough, R. and Alexandersson, M. and An, P. et al. 2002, Nature, 420, 520.

203. Gould, T.D. and Gottesman, II 2006, Genes Brain Behav, 5, 113.

204. Walters, J.T. and Owen, M.J. 2007, Mol Psychiatry, 12, 886.

205. Gottesman, II and Gould, T.D. 2003, Am J Psychiatry, 160, 636.

206. Bearden, C.E. and Freimer, N.B. 2006, Trends Genet, 22, 306.

207. Winterer, G. and Goldman, D. 2003, Brain Res Brain Res Rev, 43, 134.

208. Burdick, K.E., Lencz, T., Funke, B., Finn, C.T., Szeszko, P.R., Kane, J.M., Kucherlapati, R. and Malhotra, A.K. 2006, Hum Mol Genet, 15, 1563.

209. Ellenbroek, B.A. 2003, Behav Pharmacol, 14, 409.

210. Chen, J., Lipska, B.K. and Weinberger, D.R. 2006, Biol Psychiatry, 59, 1180.

211. Brose, N. 2008, Mol Cells, 25, 7.

212. Harrison, P.J. and Weinberger, D.R. 2005, Mol Psychiatry, 10, 40.

213. O'Tuathaigh, C.M., Babovic, D., O'Meara, G., Clifford, J.J., Croke, D.T. and Waddington, J.L. 2007, Neurosci Biobehav Rev, 31, 60.

214. Gogos, J.A. and Gerber, D.J. 2006, Trends Pharmacol Sci, 27, 226.

215. Fukui, N., Muratake, T., Kaneko, N., Amagane, H. and Someya, T. 2006, Neurosci Lett, 396, 117.

216. Gruber, O., Falkai, P., Schneider-Axmann, T., Schwab, S.G., Wagner, M. and Maier, W. 2008, J Psychiatr Res.

217. Yang, J.Z., Si, T.M., Ruan, Y., Ling, Y.S., Han, Y.H., Wang, X.L., Zhou, M., Zhang, H.Y., Kong, Q.M., Liu, C. et al. 2003, Mol Psychiatry, 8, 706.

218. Zhao, X., Shi, Y., Tang, J., Tang, R., Yu, L., Gu, N., Feng, G., Zhu, S., Liu, H., Xing, Y. et al. 2004, J Med Genet, 41, 31. 
219.Li, T., Stefansson, H., Gudfinnsson, E., Cai, G., Liu, X., Murray, R.M., Steinthorsdottir, V., Januel, D., Gudnadottir, V.G., Petursson, H. et al. 2004, Mol Psychiatry, 9, 698.

220. Munafo, M.R., Thiselton, D.L., Clark, T.G. and Flint, J. 2006, Mol Psychiatry, $11,539$.

221. Munafo, M.R., Attwood, A.S. and Flint, J. 2008, Schizophr Bull, 34, 9.

222. Li, D., Collier, D.A. and He, L. 2006, Hum Mol Genet, 15, 1995.

223. Tosato, S., Dazzan, P. and Collier, D. 2005, Schizophr Bull, 31, 613.

224. Ikeda, M., Takahashi, N., Saito, S., Aleksic, B., Watanabe, Y., Nunokawa, A., Yamanouchi, Y., Kitajima, T., Kinoshita, Y., Kishi, T. et al. 2008, Schizophr Res.

225. Duan, J., Martinez, M., Sanders, A.R., Hou, C., Krasner, A.J., Schwartz, D.B. and Gejman, P.V. 2005, Psychol Med, 35, 1599.

226. Thiselton, D.L., Webb, B.T., Neale, B.M., Ribble, R.C., O'Neill, F.A., Walsh, D., Riley, B.P. and Kendler, K.S. 2004, Mol Psychiatry, 9, 777.

227. Gardner, M., Gonzalez-Neira, A., Lao, O., Calafell, F., Bertranpetit, J. and Comas, D. 2006, Mol Psychiatry, 11, 66.

228. Corfas, G., Roy, K. and Buxbaum, J.D. 2004, Nat Neurosci, 7, 575.

229. Harrison, P.J. and Law, A.J. 2006, Biol Psychiatry, 60, 132.

230. Falls, D.L. 2003, Exp Cell Res, 284, 14.

231. Falls, D.L. 2003, J Neurocytol, 32, 619.

232. Gu, Z., Jiang, Q., Fu, A.K., Ip, N.Y. and Yan, Z. 2005, J Neurosci, 25, 4974.

233. Woo, R.S., Li, X.M., Tao, Y., Carpenter-Hyland, E., Huang, Y.Z., Weber, J., Neiswender, H., Dong, X.P., Wu, J., Gassmann, M. et al. 2007, Neuron, 54, 599.

234. Hahn, C.G., Wang, H.Y., Cho, D.S., Talbot, K., Gur, R.E., Berrettini, W.H., Bakshi, K., Kamins, J., Borgmann-Winter, K.E., Siegel, S.J. et al. 2006, Nat Med, 12, 824.

235. Li, B., Woo, R.S., Mei, L. and Malinow, R. 2007, Neuron, 54, 583.

236. Hall, J., Whalley, H.C., Job, D.E., Baig, B.J., McIntosh, A.M., Evans, K.L., Thomson, P.A., Porteous, D.J., Cunningham-Owens, D.G., Johnstone, E.C. et al. 2006, Nat Neurosci, 9, 1477.

237. Stefansson, H., Sarginson, J., Kong, A., Yates, P., Steinthorsdottir, V., Gudfinnsson, E., Gunnarsdottir, S., Walker, N., Petursson, H., Crombie, C. et al. 2003, Am J Hum Genet, 72, 83.

238. van Erp, T.G., Saleh, P.A., Huttunen, M., Lonnqvist, J., Kaprio, J., Salonen, O., Valanne, L., Poutanen, V.P., Standertskjold-Nordenstam, C.G. and Cannon, T.D. 2004, Arch Gen Psychiatry, 61, 346.

239. van Haren, N.E., Picchioni, M.M., McDonald, C., Marshall, N., Davis, N., Ribchester, T., Hulshoff Pol, H.E., Sharma, T., Sham, P., Kahn, R.S. et al. 2004, Biol Psychiatry, 56, 454.

240. Addington, A.M., Gornick, M.C., Shaw, P., Seal, J., Gogtay, N., Greenstein, D., Clasen, L., Coffey, M., Gochman, P., Long, R. et al. 2007, Mol Psychiatry, 12, 195. 241. Gerlai, R., Pisacane, P. and Erickson, S. 2000, Behav Brain Res, 109, 219.

242. O'Tuathaigh, C.M., Babovic, D., O'Sullivan, G.J., Clifford, J.J., Tighe, O., Croke, D.T., Harvey, R. and Waddington, J.L. 2007, Neuroscience, 147, 18. 
243. Rimer, M., Barrett, D.W., Maldonado, M.A., Vock, V.M. and Gonzalez-Lima, F. 2005, Neuroreport, 16, 271.

244. Reim, K., Wegmeyer, H., Brandstatter, J.H., Xue, M., Rosenmund, C., Dresbach, T., Hofmann, K. and Brose, N. 2005, J Cell Biol, 169, 669.

245. McMahon, H.T., Missler, M., Li, C. and Sudhof, T.C. 1995, Cell, 83, 111.

246. Harrison, P.J. and Eastwood, S.L. 1998, Lancet, 352, 1669.

247. Eastwood, S.L. and Harrison, P.J. 2005, Schizophr Res, 73, 159.

248. Yamauchi, Y., Qin, L.H., Nishihara, M., Sawada, K., Kato, K. and Inoue, S. 2005, Brain Res, 1056, 59.

249. Takahashi, S., Ujihara, H., Huang, G.Z., Yagyu, K.I., Sanbo, M., Kaba, H. and Yagi, T. 1999, Eur J Neurosci, 11, 2359.

250. Chubb, J.E., Bradshaw, N.J., Soares, D.C., Porteous, D.J. and Millar, J.K. 2008, Mol Psychiatry, 13, 36.

251. Hennah, W., Thomson, P., McQuillin, A., Bass, N., Loukola, A., Anjorin, A., Blackwood, D., Curtis, D., Deary, I.J., Harris, S.E. et al. 2008, Mol Psychiatry.

252. Kilpinen, H., Ylisaukko-Oja, T., Hennah, W., Palo, O.M., Varilo, T., Vanhala, R., Nieminen-von Wendt, T., von Wendt, L., Paunio, T. and Peltonen, L. 2008, Mol Psychiatry, 13, 187.

253. St Clair, D., Blackwood, D., Muir, W., Carothers, A., Walker, M., Spowart, G., Gosden, C. and Evans, H.J. 1990, Lancet, 336, 13.

254. Millar, J.K., Wilson-Annan, J.C., Anderson, S., Christie, S., Taylor, M.S., Semple, C.A., Devon, R.S., Clair, D.M., Muir, W.J., Blackwood, D.H. et al. 2000, Hum Mol Genet, 9, 1415.

255. Hennah, W., Varilo, T., Kestila, M., Paunio, T., Arajarvi, R., Haukka, J., Parker, A., Martin, R., Levitzky, S., Partonen, T. et al. 2003, Hum Mol Genet, 12, 3151.

256. Hodgkinson, C.A., Goldman, D., Jaeger, J., Persaud, S., Kane, J.M., Lipsky, R.H. and Malhotra, A.K. 2004, Am J Hum Genet, 75, 862.

257. Porteous, D.J., Thomson, P., Brandon, N.J. and Millar, J.K. 2006, Biol Psychiatry, 60, 123.

258. Duan, X., Chang, J.H., Ge, S., Faulkner, R.L., Kim, J.Y., Kitabatake, Y., Liu, X.B., Yang, C.H., Jordan, J.D., Ma, D.K. et al. 2007, Cell, 130, 1146.

259. Millar, J.K., James, R., Brandon, N.J. and Thomson, P.A. 2004, Ann Med, 36, 367.

260. Schurov, I.L., Handford, E.J., Brandon, N.J. and Whiting, P.J. 2004, Mol Psychiatry, 9, 1100.

261. Lipska, B.K., Peters, T., Hyde, T.M., Halim, N., Horowitz, C., Mitkus, S., Weickert, C.S., Matsumoto, M., Sawa, A., Straub, R.E. et al. 2006, Hum Mol Genet, 15, 1245.

262. Burdick, K.E., Hodgkinson, C.A., Szeszko, P.R., Lencz, T., Ekholm, J.M., Kane, J.M., Goldman, D. and Malhotra, A.K. 2005, Neuroreport, 16, 1399.

263. Millar, J.K., Pickard, B.S., Mackie, S., James, R., Christie, S., Buchanan, S.R., Malloy, M.P., Chubb, J.E., Huston, E., Baillie, G.S. et al. 2005, Science, 310, 1187.

264. Davis, R.L., Cherry, J., Dauwalder, B., Han, P.L. and Skoulakis, E. 1995, Mol Cell Biochem, 149-150, 271. 
265. Alberini, C.M., Ghirardi, M., Huang, Y.Y., Nguyen, P.V. and Kandel, E.R. 1995, Ann N Y Acad Sci, 758, 261.

266. Porteous, D.J. and Millar, J.K. 2006, Trends Mol Med, 12, 255.

267. Samochowiec, J., Hajduk, A., Samochowiec, A., Horodnicki, J., Stepien, G., Grzywacz, A. and Kucharska-Mazur, J. 2004, Psychiatry Res, 128, 21.

268. Hosak, L. 2007, Eur Psychiatry, 22, 276.

269. Murphy, K.C., Jones, L.A. and Owen, M.J. 1999, Arch Gen Psychiatry, 56, 940.

270. Blasi, G., Mattay, V.S., Bertolino, A., Elvevag, B., Callicott, J.H., Das, S., Kolachana, B.S., Egan, M.F., Goldberg, T.E. and Weinberger, D.R. 2005, J Neurosci, 25, 5038.

271.Egan, M.F., Goldberg, T.E., Kolachana, B.S., Callicott, J.H., Mazzanti, C.M., Straub, R.E., Goldman, D. and Weinberger, D.R. 2001, Proc Natl Acad Sci U S A, 98, 6917.

272. Bilder, R.M., Volavka, J., Czobor, P., Malhotra, A.K., Kennedy, J.L., Ni, X., Goldman, R.S., Hoptman, M.J., Sheitman, B., Lindenmayer, J.P. et al. 2002, Biol Psychiatry, 52, 701.

273. Chen, X., Wang, X., O'Neill, A.F., Walsh, D. and Kendler, K.S. 2004, Mol Psychiatry, 9, 962.

274. Shifman, S., Bronstein, M., Sternfeld, M., Pisante-Shalom, A., Lev-Lehman, E., Weizman, A., Reznik, I., Spivak, B., Grisaru, N., Karp, L. et al. 2002, Am J Hum Genet, 71, 1296.

275. Wei, J. and Hemmings, G.P. 1999, Psychiatr Genet, 9, 183.

276. Joo, E.J., Jeong, S.H., Ahn, Y.M., Lee, K.Y., Chang Yoon, S., Kim, E.J., Kim, S.U., Cho, S.C. and Sik Kim, Y. 2005, Psychiatry Res, 136, 83.

277. Munafo, M.R., Bowes, L., Clark, T.G. and Flint, J. 2005, Mol Psychiatry, 10, 765.

278. Glatt, S.J., Faraone, S.V. and Tsuang, M.T. 2003, Am J Psychiatry, 160, 469.

279. Goldberg, T.E., Egan, M.F., Gscheidle, T., Coppola, R., Weickert, T., Kolachana, B.S., Goldman, D. and Weinberger, D.R. 2003, Arch Gen Psychiatry, 60, 889.

280. Apud, J.A., Mattay, V., Chen, J., Kolachana, B.S., Callicott, J.H., Rasetti, R., Alce, G., Iudicello, J.E., Akbar, N., Egan, M.F. et al. 2007, Neuropsychopharmacology, 32, 1011.

281. Meyer-Lindenberg, A. and Weinberger, D.R. 2006, Nat Rev Neurosci, 7, 818.

282. Tunbridge, E.M., Harrison, P.J. and Weinberger, D.R. 2006, Biol Psychiatry, 60, 141.

283. Craddock, N., O'Donovan, M.C. and Owen, M.J. 2006, Schizophr Bull, 32, 9.

284. Straub, R.E., Jiang, Y., MacLean, C.J., Ma, Y., Webb, B.T., Myakishev, M.V., Harris-Kerr, C., Wormley, B., Sadek, H., Kadambi, B. et al. 2002, Am J Hum Genet, 71, 337.

285. Schwab, S.G., Knapp, M., Mondabon, S., Hallmayer, J., Borrmann-Hassenbach, M., Albus, M., Lerer, B., Rietschel, M., Trixler, M., Maier, W. et al. 2003, Am J Hum Genet, 72, 185.

286. Kirov, G., Ivanov, D., Williams, N.M., Preece, A., Nikolov, I., Milev, R., Koleva, S., Dimitrova, A., Toncheva, D., O'Donovan, M.C. et al. 2004, Biol Psychiatry, 55, 971. 
287.Liu, C.M., Liu, Y.L., Fann, C.S., Yang, W.C., Wu, J.Y., Hung, S.I., Chen, W.J., Chueh, C.M., Liu, W.M., Liu, C.C. et al. 2007, Schizophr Res, 93, 391.

288. Joo, E.J., Lee, K.Y., Jeong, S.H., Ahn, Y.M., Koo, Y.J. and Kim, Y.S. 2006, Neurosci Lett, 407, 101.

289. Datta, S.R., McQuillin, A., Puri, V., Choudhury, K., Thirumalai, S., Lawrence, J., Pimm, J., Bass, N., Lamb, G., Moorey, H. et al. 2007, Behav Brain Funct, 3, 50.

290. Benson, M.A., Newey, S.E., Martin-Rendon, E., Hawkes, R. and Blake, D.J. 2001, J Biol Chem, 276, 24232.

291. Weickert, C.S., Straub, R.E., McClintock, B.W., Matsumoto, M., Hashimoto, R., Hyde, T.M., Herman, M.M., Weinberger, D.R. and Kleinman, J.E. 2004, Arch Gen Psychiatry, 61, 544.

292. Talbot, K., Eidem, W.L., Tinsley, C.L., Benson, M.A., Thompson, E.W., Smith, R.J., Hahn, C.G., Siegel, S.J., Trojanowski, J.Q., Gur, R.E. et al. 2004, J Clin Invest, 113, 1353.

293. Olney, J.W., Newcomer, J.W. and Farber, N.B. 1999, J Psychiatr Res, 33, 523.

294. Williams, N.M., Preece, A., Morris, D.W., Spurlock, G., Bray, N.J., Stephens, M., Norton, N., Williams, H., Clement, M., Dwyer, S. et al. 2004, Arch Gen Psychiatry, 61, 336.

295. van den Oord, E.J., Sullivan, P.F., Jiang, Y., Walsh, D., O'Neill, F.A., Kendler, K.S. and Riley, B.P. 2003, Mol Psychiatry, 8, 499.

296. Fanous, A.H., van den Oord, E.J., Riley, B.P., Aggen, S.H., Neale, M.C., O'Neill, F.A., Walsh, D. and Kendler, K.S. 2005, Am J Psychiatry, 162, 1824.

297. DeRosse, P., Funke, B., Burdick, K.E., Lencz, T., Ekholm, J.M., Kane, J.M., Kucherlapati, R. and Malhotra, A.K. 2006, Am J Psychiatry, 163, 532.

298. Gornick, M.C., Addington, A.M., Sporn, A., Gogtay, N., Greenstein, D., Lenane, M., Gochman, P., Ordonez, A., Balkissoon, R., Vakkalanka, R. et al. 2005, J Autism Dev Disord, 35, 831.

299. Burdick, K.E., Goldberg, T.E., Funke, B., Bates, J.A., Lencz, T., Kucherlapati, R. and Malhotra, A.K. 2007, Schizophr Res, 89, 169.

300. Donohoe, G., Morris, D.W., De Sanctis, P., Magno, E., Montesi, J.L., Garavan, H.P., Robertson, I.H., Javitt, D.C., Gill, M., Corvin, A.P. et al. 2008, Biol Psychiatry, 63, 484.

301. Fallgatter, A.J., Herrmann, M.J., Hohoff, C., Ehlis, A.C., Jarczok, T.A., Freitag, C.M. and Deckert, J. 2006, Neuropsychopharmacology, 31, 2002.

302. Sillitoe, R.V., Benson, M.A., Blake, D.J. and Hawkes, R. 2003, J Neurosci, 23, 6576.

303. Li, W., Zhang, Q., Oiso, N., Novak, E.K., Gautam, R., O'Brien, E.P., Tinsley, C.L., Blake, D.J., Spritz, R.A., Copeland, N.G. et al. 2003, Nat Genet, 35, 84.

304. Levitt, P., Ebert, P., Mirnics, K., Nimgaonkar, V.L. and Lewis, D.A. 2006, Biol Psychiatry, 60, 534.

305. Talkowski, M.E., Chowdari, K., Lewis, D.A. and Nimgaonkar, V.L. 2006, Schizophr Bull, 32, 203.

306. Talkowski, M.E., Seltman, H., Bassett, A.S., Brzustowicz, L.M., Chen, X., Chowdari, K.V., Collier, D.A., Cordeiro, Q., Corvin, A.P., Deshpande, S.N. et al. 2006, Biol Psychiatry, 60, 152. 
307. Chen, X., Dunham, C., Kendler, S., Wang, X., O'Neill, F.A., Walsh, D. and Kendler, K.S. 2004, Am J Med Genet B Neuropsychiatr Genet, 129, 23.

308. Mirnics, K., Middleton, F.A., Stanwood, G.D., Lewis, D.A. and Levitt, P. 2001, Mol Psychiatry, 6, 293.

309. Ni, Y.G., Gold, S.J., Iredale, P.A., Terwilliger, R.Z., Duman, R.S. and Nestler, E.J. 1999, J Neurosci, 19, 3674.

310. Sobell, J.L., Richard, C., Wirshing, D.A. and Heston, L.L. 2005, Am J Med Genet B Neuropsychiatr Genet, 139, 23.

311. Ishiguro, H., Horiuchi, Y., Koga, M., Inada, T., Iwata, N., Ozaki, N., Ujike, H., Muratake, T., Someya, T. and Arinami, T. 2007, Schizophr Res, 89, 161.

312. Zhang, F., St Clair, D., Liu, X., Sun, X., Sham, P.C., Crombie, C., Ma, X., Wang, Q., Meng, H., Deng, W. et al. 2005, Genes Brain Behav, 4, 444.

313. Gold, S.J., Ni, Y.G., Dohlman, H.G. and Nestler, E.J. 1997, J Neurosci, 17, 8024.

314. Erdely, H.A., Lahti, R.A., Lopez, M.B., Myers, C.S., Roberts, R.C., Tamminga, C.A. and Vogel, M.W. 2004, Eur J Neurosci, 19, 3125.

315. Taymans, J.M., Leysen, J.E. and Langlois, X. 2003, J Neurochem, 84, 1118.

316. Geurts, M., Hermans, E. and Maloteaux, J.M. 2002, Neurosci Lett, 333, 146.

317. Saugstad, J.A., Marino, M.J., Folk, J.A., Hepler, J.R. and Conn, P.J. 1998, J Neurosci, 18, 905.

318. Bowden, N.A., Scott, R.J. and Tooney, P.A. 2007, Schizophr Res, 89, 165.

319. Cleghorn, J.M., Garnett, E.S., Nahmias, C., Brown, G.M., Kaplan, R.D., Szechtman, H., Szechtman, B., Franco, S., Dermer, S.W. and Cook, P. 1990, Br J Psychiatry, 157, 562.

320. Buckholtz, J.W., Meyer-Lindenberg, A., Honea, R.A., Straub, R.E., Pezawas, L., Egan, M.F., Vakkalanka, R., Kolachana, B., Verchinski, B.A., Sust, S. et al. 2007, J Neurosci, 27, 1584.

321. Grillet, N., Pattyn, A., Contet, C., Kieffer, B.L., Goridis, C. and Brunet, J.F. 2005, Mol Cell Biol, 25, 4221.

322. Sanders, A.R., Duan, J., Levinson, D.F., Shi, J., He, D., Hou, C., Burrell, G.J., Rice, J.P., Nertney, D.A., Olincy, A. et al. 2008, Am J Psychiatry.

323. Riley, B. and Kendler, K.S. 2006, Eur J Hum Genet, 14, 669.

324. Shirts, B.H. and Nimgaonkar, V. 2004, Curr Psychiatry Rep, 6, 303.

325. Sullivan, P.F. 2005, PLoS Med, 2, e212.

326. Bayer, T.A., Falkai, P. and Maier, W. 1999, J Psychiatr Res, 33, 543.

327. Maynard, T.M., Sikich, L., Lieberman, J.A. and LaMantia, A.S. 2001, Schizophr Bull, 27, 457.

328. Hori, T., Subramaniam, S., Srivastava, L.K. and Quirion, R. 2000, Neuropharmacology, 39, 2478.

329. Boucher, A.A., Arnold, J.C., Duffy, L., Schofield, P.R., Micheau, J. and Karl, T. 2007, Psychopharmacology (Berl), 192, 325.

330. Siren, A.L., Radyushkin, K., Boretius, S., Kammer, D., Riechers, C.C., Natt, O., Sargin, D., Watanabe, T., Sperling, S., Michaelis, T. et al. 2006, Brain, 129, 480. 


\subsection{ORIGINAL PUBLICATION}

Radyushkin K, El-Kordi A*, Boretius S, Castaneda S, Ronnenberg A, Reim K,

Bickeböller H, Frahm J, Brose $\mathrm{N}$ and Ehrenreich H (2010). Complexin2 null mutation requires a 'second hit' for induction of phenotypic changes relevant to schizophrenia. Genes, Brain and Behavior, 9:592-602.

${ }^{*}$ Equally contributing first author

\section{Personal contribution}

I was involved in the design of the study, conduction of behavioral and psychopharmacological experiments, analysis and interpretation of behavioral data, analysis and supervision of analysis of MRI data, and preparing the manuscript. 


\section{Complexin2 null mutation requires a 'second hit' for induction of phenotypic changes relevant to schizophrenia}

\author{
K. Radyushkin ${ }^{\dagger, * *, 1}$, A. El-Kordi ${ }^{\dagger, * *, 1}$, \\ S. Boretius ${ }^{\ddagger, * *}$, S. Castaneda ${ }^{\dagger}$, A. Ronnenberg ${ }^{\dagger}$, \\ K. Reim ${ }^{\S}$, H. Bickeböllerף, J. Frahm ${ }^{\ddagger, * *}$, \\ N. Brose ${ }^{\S, * * * *}$ and H. Ehrenreich ${ }^{\dagger, * * * *}$
}

\begin{abstract}
${ }^{\dagger}$ Division of Clinical Neuroscience, Max Planck Institute of Experimental Medicine, ${ }^{\ddagger}$ Biomedizinische NMR Forschungs $\mathrm{GmbH}$, Max Planck Institute for Biophysical Chemistry,

$\S$ Department of Molecular Neurobiology, Max Planck Institute of Experimental Medicine, "Department of Genetic

Epidemiology, Georg August University, and ${ }^{* *}$ DFG Center for Molecular Physiology of the Brain (CMPB), Göttingen, Germany

${ }^{1}$ These authors contributed equally to this work.

* Corresponding authors: Professor Hannelore Ehrenreich, MD, DVM, Division of Clinical Neuroscience, Max Planck Institute of Experimental Medicine, Hermann-Rein-Str. 3, 37075 Göttingen, Germany. E-mail: ehrenreich@em.mpg.de

Professor Nils Brose, PhD, Department of Molecular Neurobiology, Max Planck Institute of Experimental Medicine, Hermann-Rein-Str. 3, 37075 Göttingen, Germany. E-mail: brose@em.mpg.de
\end{abstract}

Schizophrenia is a devastating disease that affects approximately $1 \%$ of the population across cultures. Its neurobiological underpinnings are still unknown. Accordingly, animal models of schizophrenia often lack construct validity. As concordance rate in monozygotic twins amounts to only $50 \%$, environmental risk factors (e.g. neurotrauma, drug abuse, psychotrauma) likely act as necessary 'second hit' to trigger/drive the disease process in a genetically predisposed individual. Valid animal models would have to consider this genetic-environmental interaction. Based on this concept, we designed an experimental approach for modeling a schizophrenia-like phenotype in mice. As dysfunction in synaptic transmission plays a key role in schizophrenia, and complexin2 (CPLX2) gene expression is reduced in hippocampus of schizophrenic patients, we developed a mouse model with $C$ plx2 null mutation as genetic risk factor and a mild parietal neurotrauma, applied during puberty, as environmental 'second hit'. Several months after lesion, $\mathrm{Cp} / \times 2$ null mutants showed reduced pre-pulse inhibition, deficit of spatial learning and loss of inhibition after MK-801 challenge. These abnormalities were largely absent in lesioned wild-type mice and non-lesioned $C_{p} / x 2$ null mutants. Forced alternation in T-maze, object recognition, social interaction and elevated plus maze tests were unaltered in all groups. The previously reported mild motor phenotype of Cplx2 null mutants was accentuated upon lesion. MRI volumetrical analysis showed a decrease of hippocampal volume exclusively in lesioned $C p / x 2$ null mutants. These findings provide suggestive evidence for the 'second hit' hypothesis of schizophrenia and may offer new tools for the development of advanced treatment strategies.

Keywords: Animal model, cryolesion, etiology, MK801, mouse model, neurotrauma, parietal cortex, PPI, schizophrenia

Received 8 January 2010, revised 16 February 2010, 19 March 2010, and 10 April 2010, accepted for publication 13 April 2010

Schizophrenia is a frequent, debilitating, complex disease phenotype, characterized by positive, negative and cognitive symptoms (American Psychiatric Association 2000; van Os \& Kapur 2009; Wong \& Van Tol 2003) with still obscure etiology (Sawa \& Snyder, 2002). As it is debatable which constructs are central in the pathophysiology of schizophrenia, animal models with convincing construct validity (e.g. Willner 1986) have been sparse. In contrast, models with face validity, where immature rodents are exposed to lesions or pharmacological interventions, have been used for many years (for review see, e.g. Lipska 2004).

A genetic root of schizophrenia is indisputable but concordance rate in monozygotic twins is only $50 \%$ (Gottesman \& Shields 1976), pointing to additional, epigenetic/environmental risk factors (Anderson et al. 1998; Bayer et al. 1999). The 'second hit hypothesis' proposes a nongenetic risk factor ('environmental or second hit') triggering schizophrenia in predisposed individuals ('genetic or first hit'). Consequently, recent animal models unite genetic with environmental factors (for review see, e.g. Ayhan et al. 2009). Based on this 'second hit hypothesis', we designed an experimental approach for modeling schizophrenia which combines a genetic [Complexin2 (Cp/x2) null mutation] and an environmental factor (mild parietal cortical lesion during puberty) to induce schizophrenia-like features in mice.

Schizophrenia is seen as a 'disease of the synapse' (Harrison \& Weinberger 2005) bringing synaptic molecules into research focus. Among these are complexins (CPLX), presynaptic proteins that modulate neuronal exocytosis (for review see Brose 2008). They form a family of four small (134-160 residues) charged proteins, subdivided into two subfamilies: Cplx1/2 expressed in central nervous system 
and Cplx3/4 mainly in retinal ribbon synapses (McMahon et al. 1995; Reim et al. 2005). In schizophrenic postmortem brains, hippocampal CPLX2/CPLX2 expression is decreased (Harrison \& Eastwood 1998; Sawada et al. 2002). Association of single nucleotide polymorphisms in CPLX2 with schizophrenia was shown in a Korean population, adding this gene to potential risk genes (Lee et al. 2005). In Cpl $x 2^{-/-}$ mice, a mild behavioral phenotype consists of slight motor deficit and, inconsistently, impaired learning in Morris water maze (Glynn et al. 2003, 2007; Yamauchi et al. 2005).

Neurotrauma is one potential risk factor for schizophrenia (Malaspina et al. 2001; McAllister 1998), and particularly lesions affecting the right parietal lobe have been associated with schizophrenia-like psychosis (Sachdev et al. 2001; Zhang \& Sachdev 2003). As in a pivotal longitudinal study on patients with childhood onset schizophrenia, again the parietal cortex evolved as starting point of cortical gray matter loss (Thompson et al. 2001), we developed a mouse model, where a small standardized lesion is set during puberty onto the right parietal cortex. This mild cryolesion is applied stereotactically through the intact skull (no fractures) and does not cause any obvious immediate symptoms. It leaves only a very tiny, barely visible scar but initiates a bilateral neurodegenerative process very similar to that occurring endogenously in schizophrenia for as yet unknown reasons (Sargin et al. 2009; Sirén et al. 2006). Importantly, mice lesioned at a later age or in another location of the brain do not develop comparable global brain atrophy/neurodegeneration (Sirén et al. 2006). The lesion alone does not create a schizophrenic phenotype even though mild features of it are showed with careful behavioral/morphological testing, e.g. hyperactivity, cognitive dysfunction upon aging and global brain atrophy (Sargin et al. 2009; Sirén et al. 2006).

By combining Cplx2 null mutation with neurotrauma, we provide further experimental proof-of-principle that a genetic predisposition (affecting neurodevelopment) plus an environmental risk factor (inducing neurodegeneration) can give rise to prominent schizophrenia-like alterations in mouse behavior and brain morphology.

\section{Materials and methods}

\section{Animals}

All experiments were permitted by the local Animal Care and Use Committee.

CPLX2 mutant mice of mixed 129Sv-C57BI/6 background were generated by homologous recombination in embryonic stem cells as described previously (Reim et al. 2001). Behavioral experiments were performed after 10 backcrosses to the C57BL/6NCrl (Charles River Laboratories, Sulzfeld, Germany) mouse strain and employed age-matched male wild-type (WT or $\left.\mathrm{Cp}^{+/+} ; n=53\right)$ and $\mathrm{Cp} / x 2$ null mutant [knockout $(\mathrm{KO})$ or $\left.\mathrm{Cpl \times 2} 2^{-1-} ; n=65\right]$ littermates. Two cohorts of mice were used: 'cohort 1 ' $-\mathrm{WT} n=25, \mathrm{KO} n=36$; 'cohort 2' (mice were born 6 weeks later) -WT $n=28, \mathrm{KO} n=29$. Mice were housed at 4-5 per cage in a room with $12 \mathrm{~h}$ light/dark cycle (lights on at $0900 \mathrm{~h}$ ) and ad libitum access to food/water (except for the forced alternation in T-maze period; see below). The order of testing was as follows: elevated plus maze, open field, rotarod, grip strength, hind-limb clasping, social interaction, sucrose preference, pre-pulse inhibition, novel object recognition test, forced alternation in the T-maze and Morris water maze, pharmacological challenge with MK-801. All mice were used for all behavioral tests with the following exceptions: (1) for the pharmacological challenge with MK-801, a subset of mice $(n=12)$ of cohort 1 was used; (2) forced alternation in T-maze was only performed by cohort 2; (3) water maze was applied on cohort 1 and on a randomly selected subset of mice $(n=5-8)$ from cohort 2. Finally, a subset of mice ( $n=11-12$, balanced between cohorts) was examined by magnetic resonance imaging (MRI) volumetry. The order of testing was designed according to increasing invasiveness to minimize a possible influence of experimental history (Mcllwain et al. 2001). Age of mice at the beginning of testing was 16 weeks, i.e. 3 months after surgery (see below). Inter-test interval was at least 1-2 days (for timetable and illustration of the barely visible scar remaining from the cryolesion see Fig. 1a). Behavioral tests were performed in a blind fashion during the light phase of the day from 1000 until $1700 \mathrm{~h}$.

\section{Surgery}

At the age of 28 days (mouse puberty), mice were anaesthetized with an intraperitoneal injection of $0.25 \%$ tribromoethanol (Avertin) $(0.125 \mathrm{mg} / \mathrm{g})$. The parietal skull was exposed through a scalp incision and a freezing lesion was placed on the right parietal cortex (coordinates from bregma: $1.5 \mathrm{~mm}$ posterior, $1.5 \mathrm{~mm}$ lateral; Franklin \& Paxinos 1997). A cone-shaped copper cylinder with flat round tip diameter of $1 \mathrm{~mm}$ was cooled with liquid nitrogen $\left(-183^{\circ} \mathrm{C}\right)$. Its tip was stereotactically placed in direct contact with the exposed parietal skull and kept in a place for 60 seconds. Sham-operated animals went through the same procedure without cooling the metal cone. After that, the scalp was sutured and mice were kept on a heating plate $\left(37^{\circ} \mathrm{C}\right)$ until waking-up. The number of animals which was assigned to either lesion or sham-lesion was balanced between genotypes within each cohort.

\section{Behavioral testing}

\section{Elevated plus maze}

In this test of anxiety, mice were placed in the central platform, facing an open arm of the plus maze (made of gray plastic with a $5 \times 5 \mathrm{~cm}$ central platform, $30 \times 5 \mathrm{~cm}$ open arms and $30 \times 5 \times 15 \mathrm{~cm}$ closed arms; illumination 120 (x). The behavior was recorded for $5 \mathrm{~min}$ by an overhead video camera and a PC equipped with 'Viewer 2' software (Biobserve $\mathrm{GmbH}$, Bonn, Germany) to calculate the time spent in open or closed arms, distance traveled, number of arm visits and velocity. The proportion of time spent in open arms was used to estimate open arm aversion (fear equivalent).

\section{Open field}

Spontaneous activity in the open field was tested in a gray Perspex arena $(120 \mathrm{~cm}$ in diameter, $25 \mathrm{~cm}$ high; illumination $120 \mathrm{~lx})$. Mice were placed in the center and allowed to explore the open field for 7 min. The behavior was recorded by a PC-linked overhead video camera. 'Viewer 2' software was used to calculate velocity, distance traveled and time spent in central, intermediate or peripheral zones of the open field.

\section{Rotarod}

The rotarod test examines motor function, balance and coordination. It comprised a rotating drum (Ugo Basile, Comerio, Varese, Italy), which was accelerated from 4 to 40 r.p.m. over 5 min. Mice were placed individually on the drum and the latency of falling off the drum was recorded using a stop-watch. To assess motor learning, the rotarod test was repeated $24 \mathrm{~h}$ later.

\section{Grip strength}

A grip strength meter (TSE GmbH, Bad Homburg, Germany) was used to assess forelimb grip strength. Mice were lifted and held by their tail so that their forepaws could grasp a wire grid. The mice were then gently pulled backward by the tail with their posture parallel to the surface of the table until they released the grid. The peak force applied by the forelimbs of the mouse was recorded in Newton (N). Each mouse performed the test three times (with 30-min interval), and the greatest value measured was used for statistical analysis. 


\section{Radyushkin et al.}

\section{Hind-limb clasping measurement}

To measure hind-limb clasping, mice were suspended from the tail for $1 \mathrm{~min}$ to elicit the clasping phenotype, which was scored on a scale from 0 to 1 , where 0 represented no clasping, 1 represented hind paw or all paws clasping.

\section{Social interaction}

Sociability and social memory were tested as described earlier (Radyushkin et al. 2009). The social testing arena was a rectangular, three-chambered box. Each chamber was $20 \times 40 \times 22 \mathrm{~cm}$ in size. Dividing walls were made from clear Plexiglas, with rectangular openings $(35 \times 35 \mathrm{~mm})$ allowing access into each chamber. The chambers of the arena were cleaned, and fresh paper chip bedding was added between trials. The test mouse was first placed in the middle chamber and allowed to explore for $5 \mathrm{~min}$. The openings into the two-side chambers were obstructed by plastic boxes during this habituation phase. After the habituation period, an unfamiliar C57BL/6NCrl male mouse (stranger 1) without prior contact with the subject mouse was placed in one of the side chambers. The location of stranger 1 in the left vs. right side chamber was systematically alternated between trials. The stranger mouse was enclosed in a small $(60 \times 60 \times 100 \mathrm{~mm})$, rectangular wire cage, which allowed nose contact through the bars but prevented fighting. The animals serving as strangers had previously been habituated to placement in the small cage. An identical empty wire cage was placed in the opposite chamber. A heavy cup was placed on the top of each of the small wire cages to prevent climbing by the test mice. Both openings to the side chambers were then unblocked, and the subject mouse was allowed to explore the entire social test arena for a 10-min session. The amount of time spent in each chamber and the number of entries into each chamber were recorded by the video-tracking system 'Viewer 2' (Biobserve $\mathrm{GmbH}$ ). An entry was defined as all four paws in one chamber. At the end of the first 10-min trial, each mouse was tested in a second 10-min session to quantify social preference for a new stranger. A second, unfamiliar mouse (stranger 2) was placed into the previously empty wire cage. The test mouse had a choice between the first, already explored mouse (familiar stranger 1), and the novel unfamiliar mouse (new stranger 2). As described above, measures were taken of the amount of time spent in each chamber and the number of transitions between chambers of the apparatus during the second 10-min session. Based on the amount of time spent in each chamber, a 'sociability index' and a 'social memory index' (with a value of 0 meaning no preference) were calculated according to the following formulas:

$$
\begin{aligned}
& \text { Sociability index }=\left\{\left[\frac{\text { Time }_{\text {stranger }}}{\left(\text { Time }_{\text {stranger }}+\text { Time }_{\text {empty }}\right)}\right] \times 100\right\}-50 \\
& \text { Memory index }=\left\{\left[\frac{\text { Time }_{\text {novel mouse }}}{\left(\text { Time }_{\text {novel mouse }}+\text { Time }_{\text {familiar mouse }}\right)}\right] \times 100\right\}-50 \text {. }
\end{aligned}
$$

\section{Sucrose preference}

The sucrose preference test was performed as described earlier (Jamain et al. 2008) using a two-bottle procedure, during which mice had free access to both water and a sucrose solution. Animals were first habituated for $48 \mathrm{~h}$ to consume water from the two smal $(100 \mathrm{ml})$ bottles. After habituation, mice were deprived of water, and the sucrose preference was measured during the next 3 days. The first 2 days served as a habituation to sucrose solution. The results of day 3 were used for the evaluation of sucrose preference. Each day, group-housed mice were placed individually into small plastic cages and two bottles were presented to them for $60 \mathrm{~min}$ - one with tap water and one with a $2 \%$ sucrose solution. Consumption of water or sucrose solution was measured by weighing the bottles before and after the session. Bottles were counterbalanced across the left and the right sides of the cage, and their position was alternated from test to test. Sucrose preference (\%) was calculated as follows: preference $=$ [sucrose solution intake $(\mathrm{ml}) /$ total fluid intake $(\mathrm{ml})] \times 100$.

\section{Pre-pulse inhibition test}

In this test of sensorimotor gating, individual mice were placed in small metal cages $(90 \times 40 \times 40 \mathrm{~mm})$ to restrict major movements and exploratory behavior. The cages were equipped with a movable platform floor attached to a sensor that records vertical movements of the floor. The cages were placed in four sound-attenuating isolation cabinets (TSE GmbH, Bad Homburg, Germany). Startle reflexes were evoked by acoustic stimuli delivered from a loudspeaker that was suspended above the cage and connected to an acoustic generator. The startle reaction to an acoustic stimulus, which evokes a movement of the platform and a transient force resulting from this movement of the platform, was recorded with a computer during a recording window of 260 milliseconds (beginning with the onset of pre-pulse) and stored for further evaluation. The recording window was defined from the onset of the acoustic stimulus. An experimental session consisted of a 2-min habituation to $65 \mathrm{~dB}$ background white noise (continuous throughout the session), followed by a baseline recording for $1 \mathrm{~min}$ at background noise. After baseline recording, six pulse-alone trials using startle stimuli of $120 \mathrm{~dB}$ intensity and 40 milliseconds duration were applied in order to decrease the influence of within-session habituation. These data were not included in the analysis of the pre-pulse inhibition. For tests of pre-pulse inhibition, the $120 \mathrm{~dB} / 40$ milliseconds startle pulse was applied either alone or preceded by a pre-pulse stimulus of 70,75 or $80 \mathrm{~dB}$ intensity and 20 milliseconds duration. An interval of 100 milliseconds with background white noise was employed between each pre-pulse and pulse stimulus. The trials were presented in a pseudorandom order with an interval ranging from 8 to 22 seconds. The amplitude of the startle response (expressed in arbitrary units) was defined as the difference between the maximum force detected during a recording window and the force measured immediately before the stimulus onset. Amplitudes were averaged for each individual animal, separately for both types of trials (i.e. stimulus alone or stimulus preceded by a pre-pulse). Pre-pulse inhibition was calculated as the percentage of the startle response using the following formula:

$$
\begin{aligned}
& \% \text { pre-pulse inhibition } \\
& \left.\quad=100-\left[\frac{(\text { startle amplitude after pre-pulse and pulse })}{\text { (startle amplitude after pulse only) }}\right] \times 100\right] .
\end{aligned}
$$

\section{Novel object recognition test}

As a test for recognition memory, the novel object recognition test consisted of a training phase and a testing phase. First, mice were habituated to the experimental apparatus for $10 \mathrm{~min}$ in the absence of any objects. The experimental apparatus was a rectangular open field $(40 \times 40 \times 40 \mathrm{~cm})$ made of gray plastic. During the training phase, mice were placed in the experimental apparatus with one object and allowed to explore for $10 \mathrm{~min}$. The object was thoroughly cleaned between trials to make sure no olfactory cues were present. Retention was tested immediately after training and $1 \mathrm{~h}$ later. During these retention tests, the mouse explored the experimental apparatus for $10 \mathrm{~min}$ in the presence of one familiar andone novel object. An independent set of objects was used for training and testing of each retention interval. The location of the object was counterbalanced so that one-half of the animals in each group saw the novel object on the left side of the apparatus, and the other half saw the novel object on the right side of the apparatus. A mouse was scored as exploring an object when its head was oriented toward the object within a distance of $1 \mathrm{~cm}$ or closer. Preference index, a ratio of the amount of time spent exploring the new object over the total time spent exploring both objects during the retention test was used as a measure of recognition memory.

\section{Forced alternation in T-maze}

This test is used for the assessment of major deficits in working memory. The gray plastic floors of the T-maze were $5 \mathrm{~cm}$ wide. The stem of the T-maze was $35 \mathrm{~cm}$ long with a guillotine door located $20 \mathrm{~cm}$ before the arm entries. Closure of this door forms the start box. The cross-piece of the T-maze was $70 \mathrm{~cm}$ long, and at each end, there was a water well $2 \mathrm{~cm}$ in diameter and $0.75 \mathrm{~cm}$ deep. The clear Perspex walls of the maze were $15 \mathrm{~cm}$ high. For performance 
a

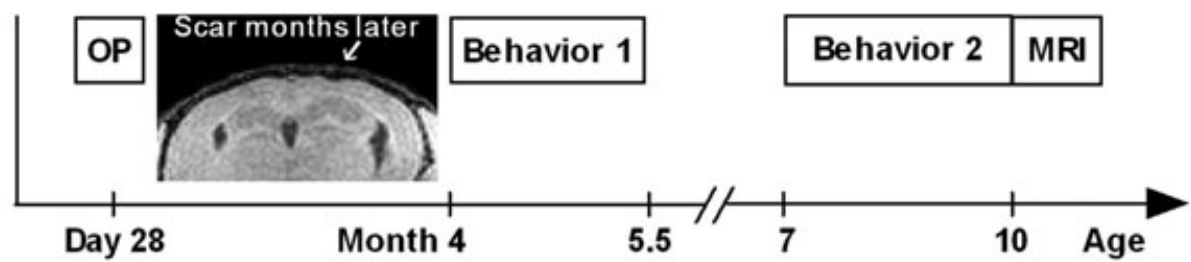

b

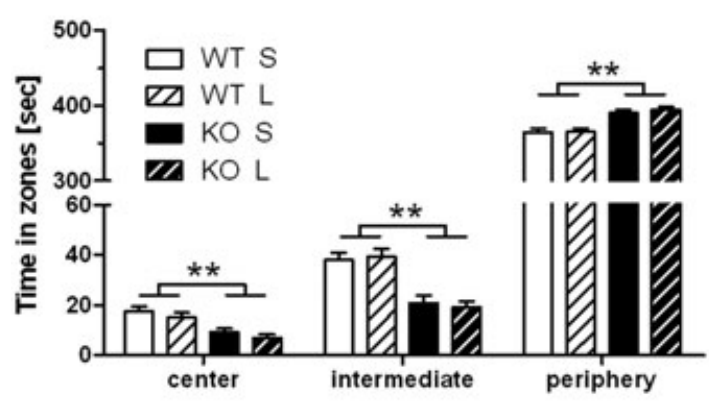

$\mathrm{d}^{200} \quad$ Elevated plus maze

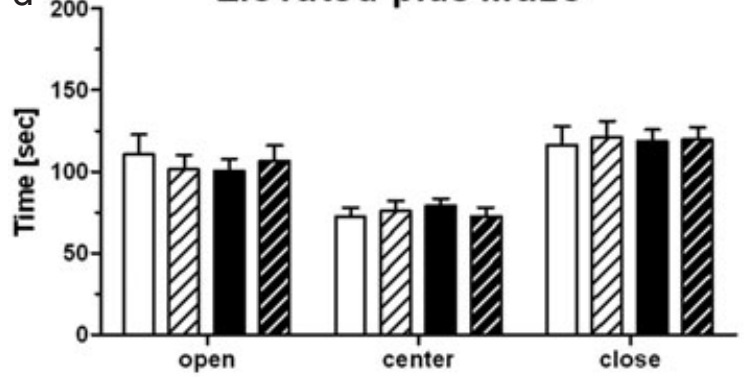

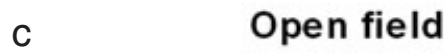
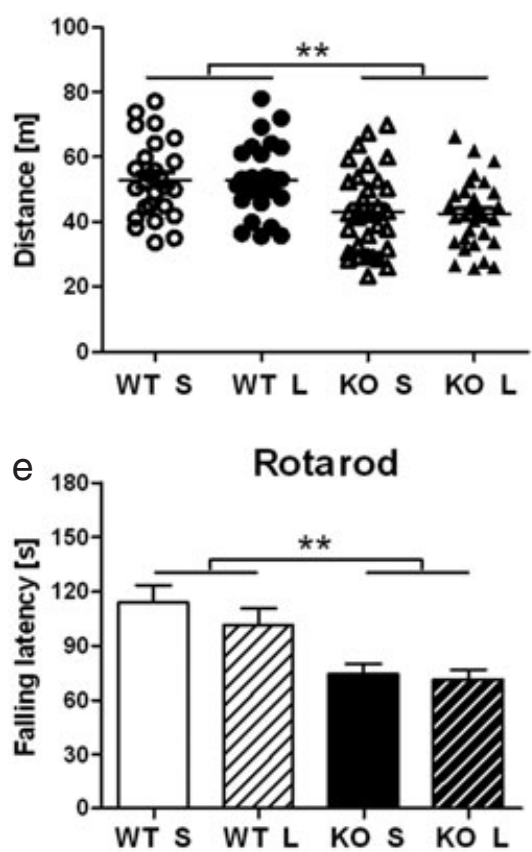

f Body weight
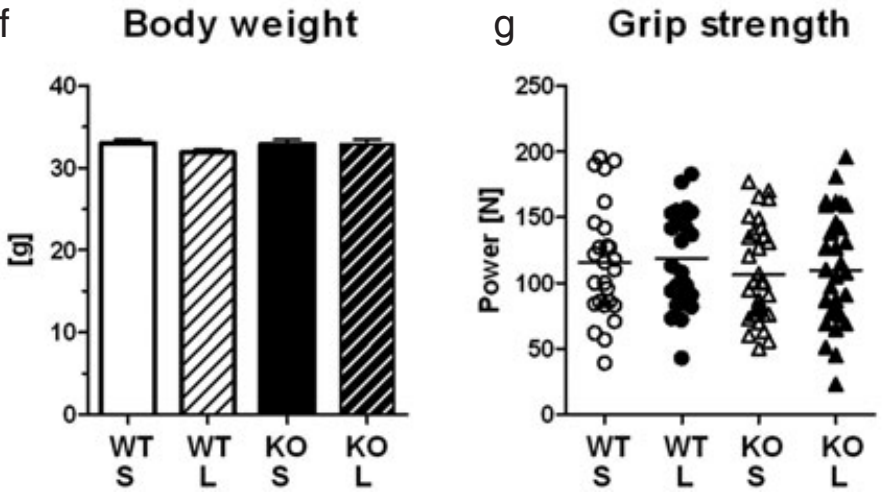

$\mathrm{h}$

Clasping

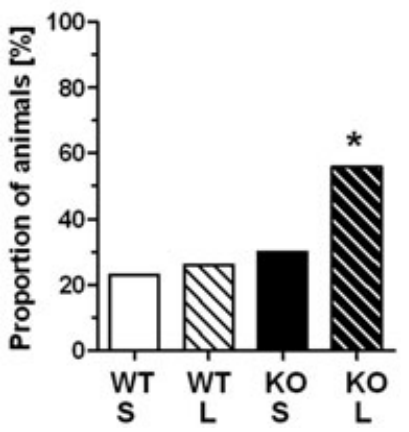

Figure 1: Experimental design (a) and effects of $\mathrm{Cpl}_{\mathrm{x} 2^{-/-}}$genotype alone and in combination with juvenile parietal neurotrauma (barely visible scar indicated by an arrow in a representative coronal MRI section in (a) as 'second hit' on readouts of general activity $(\mathbf{b}, \mathbf{c})$, anxiety $(\mathbf{b}, \mathbf{d})$, motor strength $(\mathbf{e}-\mathbf{g})$ and pathological reflexes $(\mathbf{h})$. The mild activity/motor phenotype of $\mathrm{Cp} / \times 2^{-1-}$ mice is slightly accentuated upon lesion, obvious through increased incidence of pathological reflexes. Abbreviations used in (a): $\mathrm{OP}=$ cryolesion; behavior 1 = battery of tests including elevated plus maze, open field, rotarod, grip strength, hind-limb clasping, pre-pulse inhibition, social interaction, novel object recognition, forced alternation; behavior $2=$ battery of tests including water maze and challenge with MK-801. Abbreviations used in $(b-h): K O=$ knockout; $W T=$ wild-type; $S=$ sham; $L=$ lesion; $n=26-32$ per group; mean \pm SEM presented; ${ }^{*} P<0.05 ;{ }^{*} P<0.001$. 


\section{Radyushkin et al.}

of this test, mice were kept water deprived with $1 \mathrm{~h}$ access to water per day. Habituation to the T-maze was performed over 3 days with 1 daily 5 min session during which drops of water were scattered throughout the maze. Animals were allowed to explore freely to reach the point where, at the end of habituation, they would reliably run down the stem of the maze to find water rewards in both arms. Following habituation, the animals received a total of 20 daily sessions of training. During each training session, two trials were presented with an inter-trial interval (ITI) of approximately $10 \mathrm{~min}$ Each trial consisted of twos runs. In the first run ('sample run'), liquid rewards $(0.1 \mathrm{ml} 4 \%$ sucrose solution) were placed in each well, and a wooden barrier was used to restrict access to one of the arms. As a consequence, the animal was forced to enter a pre-selected arm on each 'sample run' and then allowed to drink the reward there The animal was then picked up and confined in the start box for a specific delay. Two delays were used: 10 sessions with no delay, followed by 10 sessions with 20 seconds delay. During the second run ('test run'), the door to the start box was opened and the anima offered a free choice between the two arms of the T-maze. The criteria for selecting an arm consisted of the mouse placing a back foot in one of the arms. No retracing by the mouse was permitted If the mouse had alternated (i.e. had entered the arm not previously visited on the 'sample run'), it was allowed to drink reward before being returned to its cage. If the other arm was chosen (i.e. the same arm as visited on the 'sample run'), the mouse was confined to that arm for approximately 10 seconds and then returned to its cage. Left and right arms were used as 'sample' and 'test' arms in a counterbalanced manner. Data from five sessions were pooled together, and the percentage of correct runs was calculated and presented in graphs.

\section{Morris water maze}

Spatial learning and memory was assessed in a water maze (Morris 1984). A large circular tank (diameter $1.2 \mathrm{~m}$, depth $0.4 \mathrm{~m}$ ) was filled with opaque water $\left(25 \pm 1^{\circ} \mathrm{C}\right.$, depth $\left.0.3 \mathrm{~m}\right)$ and the escape platform $(10 \times 10 \mathrm{~cm})$ was submerged $1 \mathrm{~cm}$ below the surface. The swim patterns were monitored by a computer and the video-tracking system 'Viewer 2'. The escape latency, swim speed, path length and trajectory of swimming were recorded for each mouse. During the first 2 days, mice were trained to swim to a clearly visible platform (visible platform task) that was marked with a $15-\mathrm{cm}$ high black flag and placed pseudo-randomly in different locations across trials (non-spatial training). The extra-maze cues were hidden during these trials. After 2 days of visible platform training, hidden platform training (spatial training) was performed. For 8 days, mice were trained to find a hidden platform (i.e. the flag was removed) that was located at the center of one of the four quadrants of the pool. The location of the platform was fixed throughout testing. Mice had to navigate using extra-maze cues that were placed on the walls of the testing room. Every day, mice went through four trials with an inter-trial interval of $5 \mathrm{~min}$. The mice were placed into the pool facing the side wall randomly at one of four start locations and allowed to swim until they find the platform, or for a maximum of 90 seconds. Any mouse that failed to find the platform within 90 seconds was guided to the platform. The animal then remained on the platform for 20 seconds before being removed from the pool. The next day after completion of the hidden platform training, a probe trial was conducted in order to determine whether mice used a spatial strategy to find the platform or not. The platform was removed from the pool and the mice were allowed to swim freely for 90 seconds. The percentage of time spent in each quadrant of the pool as well as the number of times the mice crossed the former position of the hidden platform were recorded.

\section{Measurement of MK-801 induced hyperactivity} in the open field

Baseline motor activity of each mouse in open field was registered for 20 min as described above. Then mice were injected intraperitoneally with MK-801 (Sigma-Aldrich Chemie GmbH, Munich, Germany) in a volume of $10 \mathrm{ml} / \mathrm{kg}$ body weight and tested for $120 \mathrm{~min}$. We chose a dose of $0.3 \mathrm{mg} / \mathrm{kg}$, because our pilot experiments showed that this was the lowest dose causing detectable and consistent hyperactivity in non-mutant C57BL/6N mice. Right after injection, the mouse was placed into an open field and its movements were recorded for 120 min. Cumulative distance traveled over 4-min intervals was used as readout of activity.

\section{MRI volumetry}

Upon completion of the behavioral analysis, mice laged 11-12 months, Cpl $\times 2^{-1-}$ sham $n=12 ; C p / \times 2^{-1-}$ lesion as well as WT sham/lesion $n=11$ ) were anesthetized in a chamber with $5 \%$ isofluorane, intubated and kept under anesthesia with 1-1.5\% isofluorane in $\mathrm{O}_{2}$ and room air (1:1.5). In vivo brain volumetry was performed by MRI at a field strength of $2.35 \mathrm{~T}$ (Bruker Biospin, Ettlingen, Germany) using a T1-weighted three-dimensional FLASH sequence as described (Natt et al. 2002), reaching an isotropic resolution of $117 \mu \mathrm{m}$. Total brain volume (excluding olfactory bulb, cerebellum and brainstem) and, separately, the size of lateral and third ventricles, cerebellum, hippocampus and brainstem were determined by manually drawing respective regions-of-interest on up to 50 contiguous horizontal MRI sections. The experimenter performing volumetrical analyses was not aware of any group assignment of individual mice ('completely blinded').

\section{Statistical analysis}

Unless stated otherwise, the data given in figures and text are expressed as mean \pm SEM. Data were compared by either threeway analysis of variance (ANOVA) for repeated measures followed by post hoc subgroup tests (two-way ANOVAs for repeated measures for defined subgroups or, as non-parametric test, Mann-Whitney U-test to make the results even more robust against small deviations from normality) or two-way ANOVA where appropriate. As an interaction between genotype and lesion was our prior hypothesis, an interaction term was usually included in the models. Irrespective of the significance of the interaction effect, the data were further explored by separate analysis of the lesion effect within the two genotypes and of the genotype effect within the two treatment (lesion) groups. Significance obtained through this post hoc procedure was regarded as suggestive evidence for a 'second hit' in the absence of a significant genotype $\times$ lesion interaction. For analysis, SPSS v.14 software (SPSS Inc., Chicago, IL, USA) was used. Data of clasping measures were analyzed by Chi-square test. A $P$-value below 0.05 was considered to be significant. Multiple testing adjustment (Bonferroni) was applied, where indicated.

\section{Results}

\section{Accentuation of the Cplx2-/- motor phenotype upon} standardized parietal cortical neurotrauma

We first compared basic behavioral functions such as locomotor activity, anxiety and motor performance in Cplx2 null mutant vs. WT mice with or without standardized neurotrauma.

In the open field (evaluated for $7 \mathrm{~min}$ ), Cpl $\times 2^{-1-}$ in comparison with WT mice spent less time in central and intermediate zones but more in the periphery, independently of neurotrauma (two-way ANOVAs (with Bonferroni correction) for each zone: significant effect of genotype, no effect of lesion and no interaction effect; for center: $F_{1,109}=19.25$, $P<0.0001$; for intermediate: $F_{1,109}=40.27, P<0.0001$; for periphery: $F_{1,109}=37.49, \quad P<0.0001$; Fig. $\left.1 b\right)$. This behavior was associated with reduced distance traveled of the Cplx2-l-, i.e. a somewhat lower motor activity (twoway ANOVA: significant effect of genotype, $F_{1,112}=21.93$, $P<0.001$; no effects of lesion and interaction; Fig. 1c). Additional analysis of long-term open field activity obtained 
during the MK-801 challenge test (total distance traveled during the 20 min baseline evaluation before administration of MK-801) essentially confirmed these results (see below MK-801 test and Fig. 3a). The two-way ANOVA showed a significant effect of genotype $\left(F_{1,44}=14.62, P<0.0001\right)$ and no effect of lesion or interaction. Anxiety as potential explanation of the open field pattern may be excluded, because comparable levels of anxiety in all groups were found in elevated plus maze with no significant effects of genotype or lesion on the time spent in different zones leven though the amount of time spent in open arms was surprisingly large in this series of experiments, making it difficult to decisively interpret this experiment) (Fig. 1d).

In agreement with an altered motor phenotype, Cplx $2^{-/-}$ mice, independently of lesion, showed a significant decrease of their falling latency in the rotarod test for motor balance and coordination (two-way ANOVA: significant effect of genotype, $F_{1,113}=21.91, P<0.0001$; no effects of lesion and no interaction effect; Fig. 1e). A potential interfering effect of body weight with this readout could be excluded because there was no weight difference between the groups (Fig. 1f). In addition, grip strength measurements yielded no significant differences (Fig. 1g). In contrast, only in Cp/x2-/- mice, neurotrauma led to a significant increase in the proportion of animals with abnormal hind-limb clasping reflex, indicating a 'second hit' effect of lesion on motor functions ( $P=0.035$, Chi-square test; Fig. $1 \mathrm{~h}$ ). Social interaction and sucrose preference test for anhedonia did not yield differences between the groups (data not shown).

\section{Cognitive testing suggests 'second hit' effects in lesioned Cplx2-/- mice}

Neither forced alternation in T-maze nor novel object recognition tests showed any differences in performance between the groups (Fig. 2a-d). Both the tests are relatively insensitive, requiring strong deficits to show appreciable effects. In contrast, Morris water maze, a sensitive test for hippocampus-dependent learning and memory, showed a significant effect of trials (three-way ANOVA for repeated measures, $F_{7,546}=40.305, P<0.001$ ) as well as a significantly longer escape latency, particularly of $\mathrm{Cp} / \times 2^{-/-}$ mice (effect of genotype, $F_{1,78}=29.570, P<0.001$ ) with neither effect of lesion $\left(F_{1,78}=3.335, P=0.72\right)$ nor of interaction $\left(F_{1,78}=2.779, P=0.1\right)$. The plot is suggestive of a selective performance deficit of lesioned null mutants at late stages of training. Interestingly, this suggestive deficit of lesioned Cpl $\times 2^{-/-}$mice only is evident in trials 6-8, reflecting a learning impairment in the period of spatial memory formation (Fig. 2e) (Janus 2004; Wolfer et al. 1998).

Importantly, the results of the probe trial, particularly in the absence of differences at the beginning of training, provide even better support for our 'dual hit hypothesis'. Here, only lesioned Cpl $\times 2^{-1-}$ mice showed a decrease in preference for the target quadrant (Mann-Whitney $U$-test, $P=0.0071$; Fig. 2f). To exclude a potential influence of the Cpl $\times 2^{-1-}$ motor deficit on swimming speed (and therefore on escape latency), we analyzed the distance traveled by each mouse until reaching the platform, a parameter independent of swimming performance. The pattern of results was similar to the findings regarding escape latency (data not shown). Analysis of an alternative readout for spatial memory, i.e. the number of crossings of the platform site in the probe trial, yielded again the clearest defect in lesioned $\mathrm{Cp} / \times 2^{-1-}$ mice (Mann-Whitney $U$-test, $P=0.0356$; data not shown).

Pre-pulse inhibition analysis showed a significant effect of stimulus intensity (three-way ANOVA for repeated measures, $\left.F_{2,220}=136.292, \quad P<0.001\right)$ but no effect of genotype, lesion or interaction. There was a tendency for lesion $(P=0.1)$. Guided by our hypothesis of a 'dual hit' effect (genotype plus lesion), we compared in two separate twoWay ANOVAs for repeated measures (including two groups of the same genotype each) the effect of lesion vs. sham and obtained nominal significance exclusively for the combination of mutant genotype and lesion: (1) KO sham vs. KO lesion: significant effect of lesion, $F_{1,61}=4.71, P=0.03$; (2) WT sham vs. WT lesion: no effect of lesion, $F_{1,49}=0.01$, $P=0.91 \quad$ (Fig. 2g). The startle response amplitude was increased upon lesion only in Cplx2-/- (Mann-Whitney $U$-test, $P=0.0007$ ) but not in WT mice (Mann-Whitney $U$-test, $P=0.19$, Fig. $2 \mathrm{~h}$ ). Taken together, these data provide suggestive evidence for the 'second hit' hypothesis regarding higher brain functions.

\section{Increased sensitivity to MK-801 treatment in lesioned Cplx2-/- mice only}

Using three-way ANOVA for repeated measures to evaluate MK-801 results, we obtained a significant effect of time $\left(F_{29,1276}=48.418, \quad P<0.001\right)$ as well as a significant genotype $\times$ lesion interaction effect $\left(F_{1,44}=4.09, P=0.049\right)$ but no significant effect of genotype or lesion. These results allowed us to go on with a post hoc test using two separate two-way ANOVAs analyzing the effect of genotype in the lesion vs. sham paradigm separately. Although shamoperated Cplx2 $2^{-/-}$mice showed no differences in MK-801 induced hyperlocomotion in comparison to sham-operated WT mice (Fig. 3b), there was a clear 'second hit' effect detectable in lesioned $\mathrm{Cp} / \times 2^{-1-}$ mice. This group showed a relative increase in hyperlocomotion in comparison with lesioned WT mice (two-way ANOVA for repeated measures: significant effect of genotype, $F_{1,22}=5.79, \quad P=0.024$; significant effect of interaction, $F_{29,638}=3.29, P<0.0001$; Fig. 3c). When looking at sham vs. lesion groups of each genotype separately, it becomes obvious that MK-801 leads to reduced hyperactivity in lesioned wild-types while in lesioned mutants, this effect is absent, explaining the relative hyperactivity ('loss of inhibition') seen in Fig. 3.

\section{Decrease of total hippocampal volume exclusively in lesioned Cplx2-/- mice}

$\mathrm{MRI}$ volumetrical analyses failed to uncover significant differences between genotypes, independent of lesion, regarding whole brain, lateral and third ventricles, brainstem and cerebellum. The results on total brain matter (total brain circumference minus cerebellum, olfactory bulb, brain stem and ventricles) are shown in Fig. 3d. In agreement with our previously published data (Sirén et al., 2006) there is a strong tendency of a reduction in brain matter in lesioned wild-type 

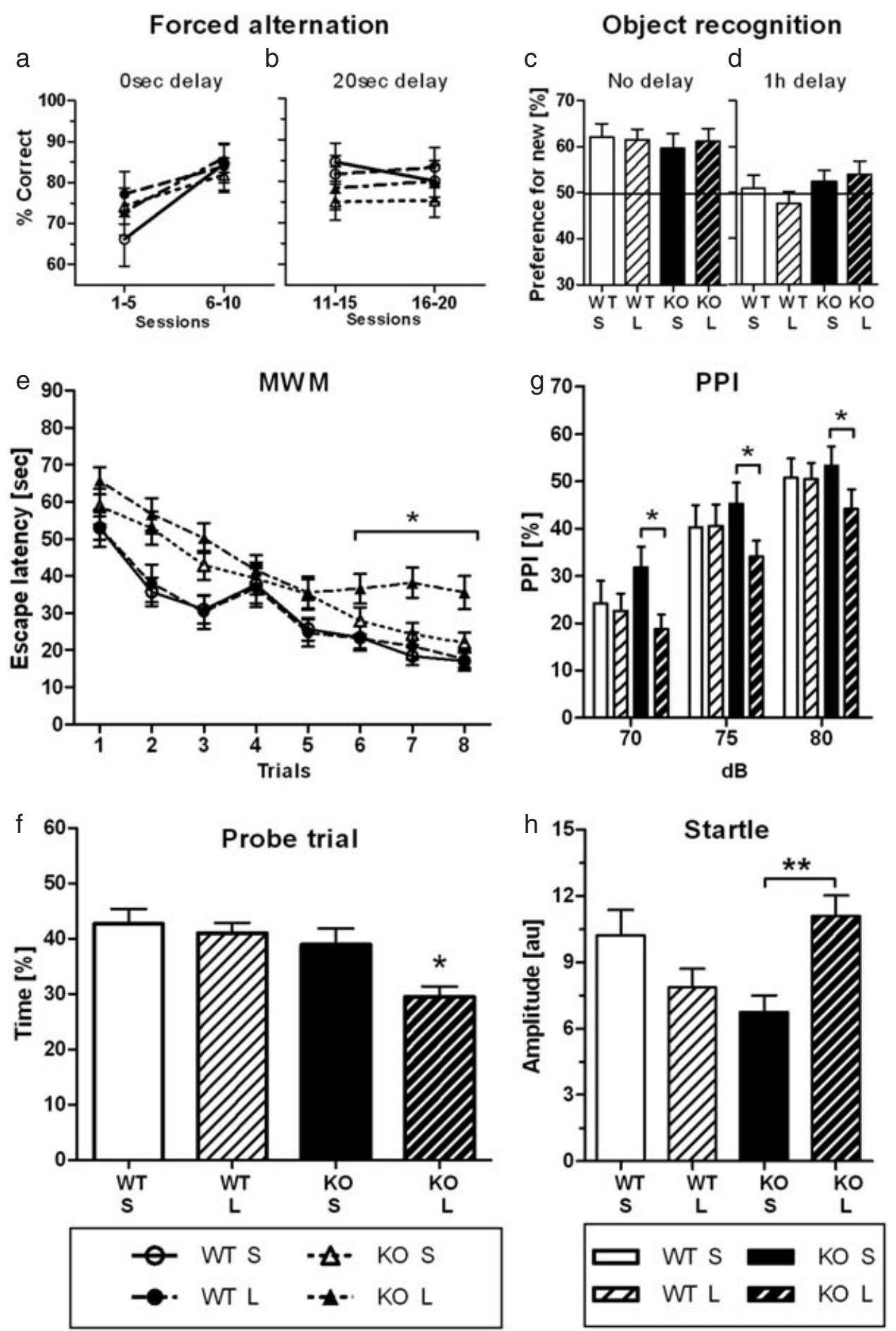

Figure 2: Effects of $\mathrm{Cpl}_{\mathbf{3} 2^{-/-}}$genotype alone and in combination with juvenile parietal neurotrauma as 'second hit' on readouts of cognitive performance $(\mathbf{a}-\mathbf{f})$ and sensorimotor gating $(\mathbf{g}, \mathbf{h})$. Whereas the relatively insensitive tests, forced alternation in T-maze (a, b) and object recognition (c, d), do not show differences between the groups, there is a remarkable deficit in spatial learning and memory in Morris water maze as well as in PPI, particularly pronounced upon dual hit (e-h). Abbreviations: MWM $=$ Morris water maze; $\mathrm{PPI}=$ pre-pulse inhibition; $\mathrm{KO}=$ knockout; $\mathrm{WT}=$ wild-type; $\mathrm{S}=$ sham; $\mathrm{L}=$ lesion; $n=17-22$ per group; mean \pm SEM presented; ${ }^{*} P<0.05 ;{ }^{*} P<0.01$ ). Note that data similar to those shown in (e), obtained with a different cohort of mice, have been included in a human genetic paper, dealing with the modifier role of CPLX2 on cognition in schizophrenia (Begemann et al. 2010). 

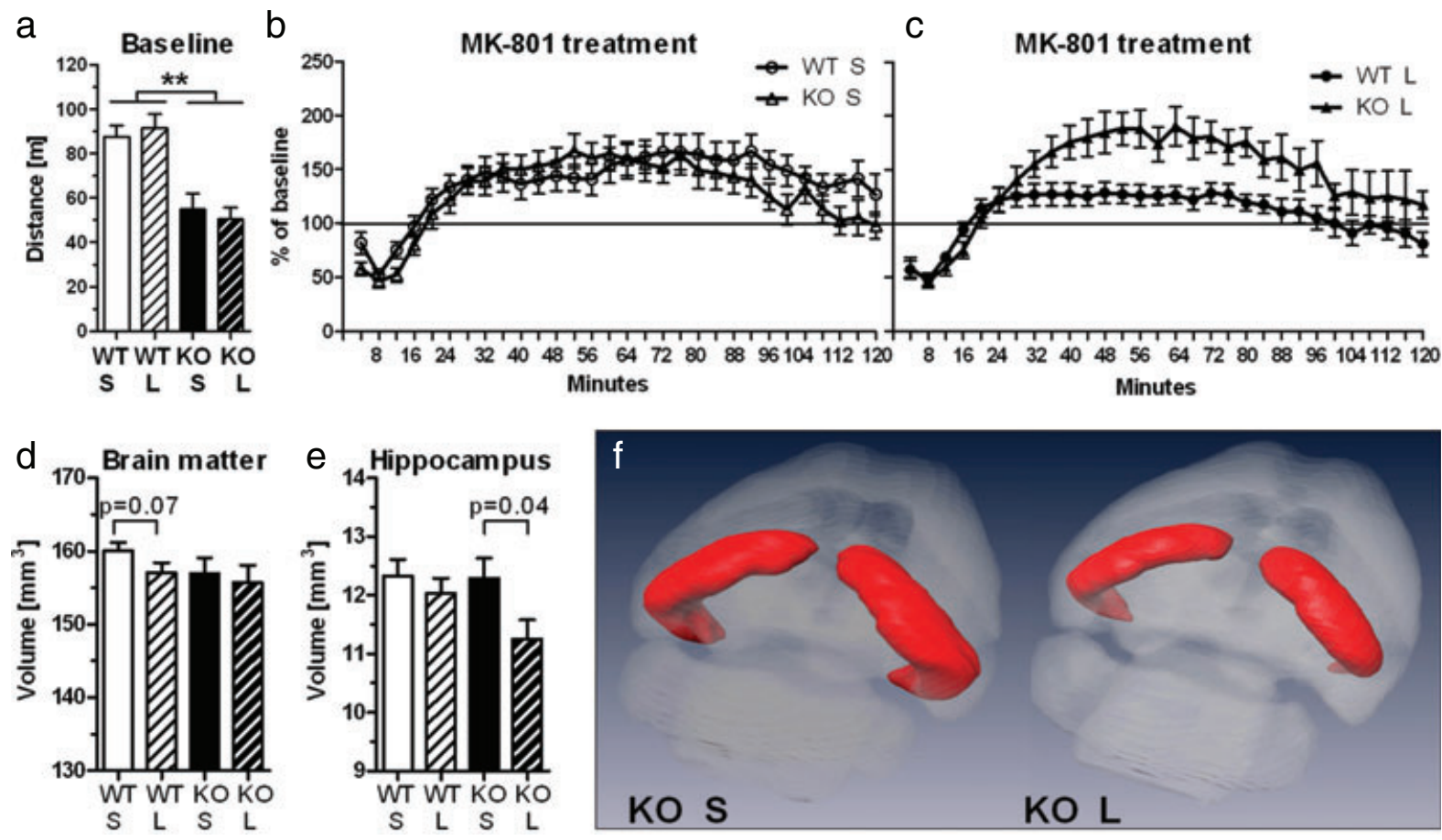

Figure 3: Effects of $C p / \times 2^{-/-}$genotype alone and in combination with juvenile parietal neurotrauma as 'second hit' on pharmacological challenge with the NMDA antagonist MK-801 (a-c), on total brain matter (d) and on hippocampal volume (e, f). Cplx $2^{-1-}$ mice, regardless of lesion, show decreased activity during baseline evaluation (20 min) before administration of MK-801 (a). Only lesioned $C p / \times 2^{-/-}$mice exhibit relative hyperactivity upon MK-801 (b, c). There is a strong tendency of a reduction in brain matter in lesioned wild-type as compared with non-lesioned wild-type mice. In contrast, non-lesioned Cp/x2 ${ }^{-1-}$ mice show a tendency of decreased brain matter that is not further reduced by lesion (d). Exclusively lesioned $C p / \times 2^{-/-}$mice have a decreased hippocampal volume (e, f). Abbreviations: $\mathrm{KO}=$ knockout; $\mathrm{WT}=$ wild-type; $\mathrm{S}=$ sham; $\mathrm{L}=$ lesion; $n=11-12$ per group; mean $\pm \mathrm{SEM}$ presented; ${ }^{*} P<0.05 ;{ }^{*} P<0.0001$.

as compared with non-lesioned wild-type mice. In contrast, non-lesioned Cplx $2^{-/-}$mice show a tendency of decreased brain matter that is not further reduced by lesion. However, using a two-way ANOVA, we obtained a significant effect of lesion $\left(F_{1,41}=4.76, P<0.03\right)$ on total hippocampal volume (Fig. 3e,f). Post hoc tests showed that the hippocampal volume was significantly reduced exclusively in lesioned Cplx2 ${ }^{-/-}$(Mann-Whitney U-test, $P=0.045$, Fig. 3e) but not in lesioned WT mice (Mann-Whitney $U$-test, $P=0.69$ ).

\section{Discussion}

We report here that the exposure of juvenile mice, carrying a null mutation of $\mathrm{Cp} / \times 2$, to a small parietal cortical lesion leads to the development of a phenotype relevant to schizophrenia. Cplx2 encodes a synaptic protein crucial for neurotransmitter release and may represent a probable schizophrenia risk gene. Mild parietal neurotrauma around puberty is one of the several potential environmental factors, acting as 'second hit'. The phenotype resulting from the combination of these two 'hits' includes alterations in sensorimotor gating, selective deficiency in learning and memory functions, a slight accentuation of the genotype-associated motor symptoms, loss of inhibition/relative hyperactivity upon NMDA receptor antagonism and a reduction in total hippocampal volume. Although the phenotype effects are mostly small and statistical support for a genotype $x$ lesion interaction is suggestive rather than conclusive despite large group sizes, chance effects are very unlikely because all observed effects point in the same direction.

A mild behavioral phenotype in $C p / \times 2^{-1-}$ mice has been reported earlier, comparable with the sham-operated Cplx2-/- group in the present work, comprising mainly a motor deficit in rotarod and open field and, inconsistently, a somewhat impaired learning curve in Morris water maze (Glynn etal. 2003, 2007; Yamauchi et al. 2005). In more schizophrenia-relevant tests, such as pre-pulse inhibition (PPI) of the startle response and pharmacological challenge with the NMDA receptor antagonist MK-801, the mutant genotype alone, i.e. sham-operated $\mathrm{Cp} / \times 2^{-1-}$ mice, showed no alterations as compared with WT mice. As an environmental 'second hit', we applied a small standardized right parietal cortical neurotrauma to juvenile mice (Sargin et al., 2009; Sirén et al., 2006). Again, consequences of this mild trauma alone, i.e. in the absence of a modifying genotype, are minor (Sargin et al., 2009; Sirén et al., 2006). 


\section{Radyushkin et al.}

This was also the case for lesioned WT mice in the present study, reflected by a tendency of reduced startle amplitude and number of visits to the former platform location in the Morris water maze probe trial.

The most clear-cut results, collectively resembling phenotypic changes relevant to schizophrenia, were obtained with the experimental group that combined effects of the genetic factor (null mutation of the Cplx2 gene) and the environmental factor (cryolesion as experimental neurotrauma) in the sense of a 'second hit': (1) deficit in PPI, a widely accepted readout of sensorimotor gating, frequently found in schizophrenic patients (Braff \& Geyer 1990; Swerdlow \& Geyer 1998), emerged in Cplx2 ${ }^{-/-}$mice only upon neurotrauma. (2) Similarly, the most prominent phenotype in learning and memory functions was observed upon dual hit. Although crude learning and memory tests, i.e. forced alternation in T-maze and novel object recognition, did not yield differences between the groups, only Morris water maze performance showed the predicted deficit in lesioned null mutant Cplx2 mice. (3) Lesioned Cpl $\times 2^{-/-}$mice displayed an exacerbation of their motor phenotype, reflected by a significantly higher incidence of abnormal hind-limb clasping. Even though pathological motor reflexes do not belong to the core symptoms of schizophrenia, they are among the so-called 'neurological soft signs' of the disease (Chan \& Gottesman 2008; Heinrichs \& Buchanan 1988). (4) Only lesioned Cpl $\times 2^{-1-}$ mice responded to the NMDA receptor antagonist MK-801 with a relative hyperactivity ('loss of inhibition') in the open field. This pharmacological challenge is regarded as another schizophrenia-related readout (Carlsson et al., 1999; Geyer \& Markou, 1995; O'Neill \& Bolger 1989). In fact, NMDA receptor antagonists such as phencyclidine or ketamine induce transient schizophrenia-like cognitive dysfunctions and perceptual alterations in healthy volunteers (Adler et al. 1999; Lahti et al. 2001; Malhotra et al. 1996), and exacerbate symptoms of schizophrenia in patients (Lahti et al. 2001; Malhotra et al. 1997). Among others, these findings gave rise to the NMDA receptor hypofunction hypothesis of schizophrenia (e.g. reviewed by Gunduz-Bruce 2009; Olney et al. 1999). This hypothesis proposes that NMDA receptor blockade disrupts NMDA receptor-mediated stimulation of GABAergic, serotonergic and noradrenergic inhibitory neurons, leading to 'loss of inhibition' in major excitatory pathways (Coyle 2004; Olney et al. 1999). (5) A significant increase of the baseline startle response was only found in $\mathrm{Cp} / \times 2^{-1-}$ mice after juvenile lesion. Although there is accumulating evidence (Csomor et al. 2008; Yee et al. 2005) assuming a dependency of \%PPI from baseline startle response, there is a wealth of literature that argues against such a dependency (Blumenthal et al. 2004; Ison et al. 1997; Yee et al. 2004). Whether baseline startle response determines \% PPI or not, together with the relative hyperactivity to a subthreshold dose of MK-801 in the open field, the significant increase in the baseline startle amplitude in Cplx2-/- mice after juvenile lesion additionally supports the idea of 'loss of inhibition' in these animals. In fact, compounds assumed to produce positive-like symptoms in mice (e.g. MK-801, PCP) have also been reported to increase the baseline startle response (Geyer et al. 1984; Karl et al. 2010). (6) Finally, the reduction in hippocampal volume, a typical finding in schizophrenia (Hyde \& Weinberger
1990; Shenton et al. 2001) is found exclusively in the dual hit group. Only readouts of negative symptoms (social withdrawal, anhedonia) were not observed in any of the groups. It should clearly be noted; however, that even the leading disease classification system (DSM-IV-TR, American Psychiatric Association 2000) does not require the presence of all symptom complexes for diagnosing schizophrenia.

In an experimental attempt similar to the one presented here, Inoue and colleagues had combined early maternal stress with Cplx2 null mutation, in order to investigate the gene $x$ environment interaction hypothesis of schizophrenia (Yamauchi et al. 2005). They found that after early maternal deprivation, Cplx2 null mutant mice developed a decrease in hippocampal long-term potentiation (LTP) and a spatial learning deficit in Morris water maze. Other schizophrenia typical readouts, supporting the validity of the model for this disease, however, were not reported in this study.

Taken together, the model of a gene $x$ environment interaction presented here results in the development of three of four lead symptom complexes of schizophrenia: positive symptoms, cognitive symptoms and brain morphology changes. The findings (1) suggest that the combination of a Cpl $\times 2^{-1-}$ mutation with juvenile parietal neurotrauma may represent a novel animal model for schizophrenia with higher construct validity than most related models tested so far and (2) provide experimental support for the 'second hit' hypothesis of schizophrenia etiology. Although the mechanisms underlying the gene $x$ environment interactions observed here are unclear at present, this novel mouse model offers an important conceptual basis for further studies on the biological processes involved in these interactions that ultimately cause schizophrenia-related brain dysfunctions.

Likely, there are many different other ways of combining genetic and environmental risk in mice to create phenotypic changes relevant for schizophrenia (for review, see, e.g. Ayhan etal. 2009). This is obviously what happens in the human population and explains why no major disease genes have been identified in schizophrenia. The gene $\times$ environment approach; however, will be pivotal for understanding the pathophysiology of the disease. Different gene $x$ environment interactions, resulting in a schizophrenia-like phenotype, will ultimately have to be compared to allow extracting common denominators of disease onset/progression.

\section{References}

Adler, C.M., Malhotra, A.K., Elman, I., Goldberg, T., Egan, M., Pickar, D. \& Breier, A. (1999) Comparison of ketamine-induced thought disorder in healthy volunteers and thought disorder in schizophrenia. Am J Psychiatry 156, 1646-1649.

American Psychiatric Association. (2000) Diagnostic and Statistical Manual of Mental Disorders: DSM-IV-TR, 4th edn. American Psychiatric Association, Washington, DC

Anderson, J.E., O'Donnell, B.F., McCarley, R.W. \& Shenton, M.E. (1998) Progressive changes in schizophrenia: do they exist and what do they mean? Restor Neurol Neurosci 12, 175-184.

Ayhan, Y., Sawa, A., Ross, C.A. \& Pletnikov, M.V. (2009) Animal models of gene-environment interactions in schizophrenia. Behav Brain Res 204, 274-281. 
Bayer, T.A., Falkai, P. \& Maier, W. (1999) Genetic and non-genetic vulnerability factors in schizophrenia: the basis of the 'two hit hypothesis'. J Psychiatr Res 33, 543-548.

Begemann, M., Klaus, S., Papiol, S., et al. (2010) Complexin2 gene polymorphisms modify cognitive performance in schizophrenia. Arch Gen Psychiatry, in press.

Blumenthal, T.D., Elden, A. \& Flaten, M.A. (2004) A comparison of several methods used to quantify prepulse inhibition of eyeblink responding. Psychophysiology 41, 326-332.

Braff, D.L. \& Geyer, M.A. (1990) Sensorimotor gating and schizophrenia. Human and animal model studies. Arch Gen Psychiatry 47, 181-188.

Brose, N. (2008) For better or for worse: complexins regulate SNARE function and vesicle fusion. Traffic 9, 1403-1413.

Carlsson, A., Hansson, L.O., Waters, N. \& Carlsson, M.L. (1999) A glutamatergic deficiency model of schizophrenia. Br J Psychiatry Supp/ 37, 2-6.

Chan, R.C. \& Gottesman, II (2008) Neurological soft signs as candidate endophenotypes for schizophrenia: a shooting star or a Northern star? Neurosci Biobehav Rev 32, 957-971.

Coyle, J.T. (2004) The GABA-glutamate connection in schizophrenia: which is the proximate cause? Biochem Pharmacol 68, $1507-1514$.

Csomor, P.A., Yee, B.K., Vollenweider, F.X., Feldon, J., Nicolet, T. \& Quednow, B.B. (2008) On the influence of baseline startle reactivity on the indexation of prepulse inhibition. Behav Neurosci 122, 885-900

Franklin, K.B.J. \& Paxinos, G. (1997) The Mouse Brain in Stereotaxic Coordinates. Academic Press, San Diego.

Geyer, M.A. \& Markou, A. (1995) Animal models of psychiatric disorders. In Bloom, F. \& Kupfer, D.J. (eds), Psychopharmacology: The Fourth Generation of Progress. Raven Press, New York, pp. xlii, 2002.

Geyer, M.A., Segal, D.S. \& Greenberg, B.D. (1984) Increased startle responding in rats treated with phencyclidine. Neurobehav Toxicol Teratol 6, 161-164.

Glynn, D., Bortnick, R.A. \& Morton, A.J. (2003) Complexin II is essential for normal neurological function in mice. Hum Mol Genet 12, 2431-2448.

Glynn, D., Reim, K., Brose, N. \& Morton, A.J. (2007) Depletion of Complexin II does not affect disease progression in a mouse model of Huntington's disease (HD): support for role for complexin II in behavioural pathology in a mouse model of HD. Brain Res Bull 72, 108-120.

Gottesman, II \& Shields, J. (1976) A critical review of recent adoption, twin, and family studies of schizophrenia: behavioral genetics perspectives. Schizophr Bull 2, 360-401.

Gunduz-Bruce, H. (2009) The acute effects of NMDA antagonism: from the rodent to the human brain. Brain Res Rev 60, 279-286.

Harrison, P.J. \& Eastwood, S.L. (1998) Preferential involvement of excitatory neurons in medial temporal lobe in schizophrenia. Lancet 352, 1669-1673.

Harrison, P.J. \& Weinberger, D.R. (2005) Schizophrenia genes, gene expression, and neuropathology: on the matter of their convergence. Mol Psychiatry 10, 40-68; image 45.

Heinrichs, D.W. \& Buchanan, R.W. (1988) Significance and meaning of neurological signs in schizophrenia. Am J Psychiatry 145, $11-18$.

Hyde, T.M. \& Weinberger, D.R. (1990) The brain in schizophrenia. Semin Neurol 10, 276-286.

Ison, J.R., Bowen, G.P., Pak, J. \& Gutierrez, E. (1997) Changes in the strength of prepulse inhibition with variation in the startle baseline associated with individual differences and with old age in rats and mice. Psychobiology 25, 266-274.

Jamain, S., Radyushkin, K., Hammerschmidt, K., Granon, S. Boretius, S., Varoqueaux, F., Ramanantsoa, N., Gallego, J., Ronnenberg, A., Winter, D., Frahm, J., Fischer, J., Bourgeron, T., Ehrenreich, H. \& Brose, N. (2008) Reduced social interaction and ultrasonic communication in a mouse model of monogenic heritable autism. Proc Natl Acad Sci U S A 105, 1710-1715.
Janus, C. (2004) Search strategies used by APP transgenic mice during navigation in the Morris water maze. Learn Mem 11, 337-346.

Karl, T., Chesworth, R., Duffy, L. \& Herzog, H. (2010) Schizophreniarelevant behaviours in a genetic mouse model for $\mathrm{Y} 2$ deficiency. Behav Brain Res 207, 434-440.

Lahti, A.C., Weiler, M.A., Tamara Michaelidis, B.A., Parwani, A. \& Tamminga, C.A. (2001) Effects of ketamine in normal and schizophrenic volunteers. Neuropsychopharmacology 25, 455-467.

Lee, H.J., Song, J.Y., Kim, J.W., Jin, S.Y., Hong, M.S., Park, J.K., Chung, J.H., Shibata, H. \& Fukumaki, Y. (2005) Association study of polymorphisms in synaptic vesicle-associated genes, SYN2 and CPLX2, with schizophrenia. Behav Brain Funct 1, 15.

Lipska, B.K. (2004) Using animal models to test a neurodevelopmental hypothesis of schizophrenia. J Psychiatry Neurosci 29 282-286.

Malaspina, D., Goetz, R.R., Friedman, J.H., Kaufmann, C.A., Faraone, S.V., Tsuang, M., Cloninger, C.R., Nurnberger, J.I. Jr \& Blehar, M.C. (2001) Traumatic brain injury and schizophrenia in members of schizophrenia and bipolar disorder pedigrees. Am J Psychiatry 158, 440-446.

Malhotra, A.K., Pinals, D.A., Adler, C.M., Elman, I., Clifton, A., Pickar, D. \& Breier, A. (1997) Ketamine-induced exacerbation of psychotic symptoms and cognitive impairment in neuroleptic-free schizophrenics. Neuropsychopharmacology 17, 141-150.

Malhotra, A.K., Pinals, D.A., Weingartner, H., Sirocco, K., Missar, C.D., Pickar, D. \& Breier, A. (1996) NMDA receptor function and human cognition: the effects of ketamine in healthy volunteers. Neuropsychopharmacology 14, 301-307.

McAllister, T.W. (1998) Traumatic brain injury and psychosis: what is the connection? Semin Clin Neuropsychiatry 3, 211-223.

Mcllwain, K.L., Merriweather, M.Y., Yuva-Paylor, L.A. \& Paylor, R. (2001) The use of behavioral test batteries: effects of training history. Physiol Behav 73, 705-717.

McMahon, H.T., Missler, M., Li, C. \& Sudhof, T.C. (1995) Complexins: cytosolic proteins that regulate SNAP receptor function. Cell 83, 111-119.

Morris, R. (1984) Developments of a water-maze procedure for studying spatial learning in the rat. J Neurosci Methods 11, 47-60.

Natt, O., Watanabe, T., Boretius, S., Radulovic, J., Frahm, J. \& Michaelis, T. (2002) High-resolution 3D MRI of mouse brain reveals small cerebral structures in vivo. J Neurosci Methods $\mathbf{1 2 0}$ 203-209.

O'Neill, S.K. \& Bolger, G.T. (1989) Phencyclidine and MK-801: a behavioral and neurochemical comparison of their interactions with dihydropyridine calcium antagonists. Brain Res Bull 22, 611-616.

Olney, J.W., Newcomer, J.W. \& Farber, N.B. (1999) NMDA receptor hypofunction model of schizophrenia. J Psychiatr Res $\mathbf{3 3}$ 523-533.

van Os, J. \& Kapur, S. (2009) Schizophrenia. Lancet 374 635-645.

Radyushkin, K., Hammerschmidt, K., Boretius, S., Varoqueaux, F. El-Kordi, A., Ronnenberg, A., Winter, D., Frahm, J., Fischer, J., Brose, N. \& Ehrenreich, H. (2009) Neuroligin-3-deficient mice: model of a monogenic heritable form of autism with an olfactory deficit. Genes Brain Behav 8, 416-425.

Reim, K., Mansour, M., Varoqueaux, F., McMahon, H.T., Sudhof, T.C., Brose, N. \& Rosenmund, C. (2001) Complexins regulate a late step in $\mathrm{Ca} 2+-$ dependent neurotransmitter release. Cell 104, 71-81.

Reim, K., Wegmeyer, H., Brandstatter, J.H., Xue, M., Rosenmund, C. Dresbach, T., Hofmann, K. \& Brose, N. (2005) Structurally and functionally unique complexins at retinal ribbon synapses. J Cell Biol 169, 669-680.

Sachdev, P., Smith, J.S. \& Cathcart, S. (2001) Schizophrenia-like psychosis following traumatic brain injury: a chart-based descriptive and case-control study. Psychol Med 31, 231-239. 


\section{Radyushkin et al.}

Sargin, D., Hassouna, I., Sperling, S., Sirén, A.L. \& Ehrenreich, H. (2009) Uncoupling of neurodegeneration and gliosis in a murine model of juvenile cortical lesion. Glia 57, 693-702.

Sawa, A. \& Snyder, S.H. (2002) Schizophrenia: diverse approaches to a complex disease. Science 296, 692-695.

Sawada, K., Young, C.E., Barr, A.M., Longworth, K., Takahashi, S., Arango, V., Mann, J.J., Dwork, A.J., Falkai, P., Phillips, A.G. \& Honer, W.G. (2002) Altered immunoreactivity of complexin protein in prefrontal cortex in severe mental illness. Mol Psychiatry $\mathbf{7}$, 484-492.

Shenton, M.E., Dickey, C.C., Frumin, M. \& McCarley, R.W. (2001) A review of MRl findings in schizophrenia. Schizophr Res $\mathbf{4 9}$ $1-52$.

Sirén, A.L., Radyushkin, K., Boretius, S., Kammer, D., Riechers, C.C., Natt, O., Sargin, D., Watanabe, T., Sperling, S., Michaelis, T., Price, J., Meyer, B., Frahm, J. \& Ehrenreich, H. (2006) Global brain atrophy after unilateral parietal lesion and its prevention by erythropoietin. Brain 129, 480-489.

Swerdlow, N.R. \& Geyer, M.A. (1998) Using an animal model of deficient sensorimotor gating to study the pathophysiology and new treatments of schizophrenia. Schizophr Bull 24 285-301.

Thompson, P.M., Vidal, C., Giedd, J.N., Gochman, P., Blumenthal, J. Nicolson, R., Toga, A.W. \& Rapoport, J.L. (2001) Mapping adolescent brain change reveals dynamic wave of accelerated gray matter loss in very early-onset schizophrenia. Proc Natl Acad Sci U S A 98, 11650-11655.

Willner, P. (1986) Validation criteria for animal models of human mental disorders: learned helplessness as a paradigm case. Prog Neuropsychopharmacol Biol Psychiatry 10, 677-690.
Wolfer, D.P., Stagljar-Bozicevic, M., Errington, M.L. \& Lipp, H.P. (1998) Spatial memory and learning in transgenic mice: fact or artifact? News Physiol Sci 13, 118-123.

Wong, A.H. \& Van Tol, H.H. (2003) Schizophrenia: from phenomenology to neurobiology. Neurosci Biobehav Rev 27, 269-306.

Yamauchi, Y., Qin, L.H., Nishihara, M., Sawada, K., Kato, K. \& Inoue, S. (2005) Vulnerability of synaptic plasticity in the complexin II knockout mouse to maternal deprivation stress. Brain Res 1056 $59-67$.

Yee, B.K., Chang, T., Pietropaolo, S. \& Feldon, J. (2005) The expression of prepulse inhibition of the acoustic startle reflex as a function of three pulse stimulus intensities, three prepulse stimulus intensities, and three levels of startle responsiveness in C57BL6/J mice. Behav Brain Res 163, 265-276.

Yee, B.K., Russig, H. \& Feldon, J. (2004) Apomorphine-induced prepulse inhibition disruption is associated with a paradoxical enhancement of prepulse stimulus reactivity. Neuropsychopharmacology 29, 240-248.

Zhang, Q. \& Sachdev, P.S. (2003) Psychotic disorder and traumatic brain injury. Curr Psychiatry Rep 5, 197-201.

\section{Acknowledgements}

This study was supported by the Max Planck Society and the DFG Center for Molecular Physiology of the Brain (CMPB). The staff of the Transgenic Animal Facility at the Max Planck Institute for Experimental Medicine is gratefully acknowledged for excellent technical assistance. 


\section{EFFECT OF EPO ON HIGHER BRAIN FUNCTIONS IN MICE}

\subsection{OVERVIEW OF PROJECT II}

Since the introduction of the hematopoietic growth factor erythropoietin (EPO) to clinical use - over 20 years ago - to treat patients suffering from anemic conditions, reports have been accumulating stating improvement of cognitive performance of the treated individuals. Initially, this improvement was attributed to anemia correction with subsequently enhanced tissue oxygenation also in the central nervous system (Jelkmann, 2005). However, after discovering that EPO and its receptor (EPOR) are produced by and act on cells of the nervous system (Masuda et al., 1993; Masuda et al., 1994) several reports on neuroprotective and neuroregenerative effects of EPO in rodent models of neurological diseases followed (for review, see Brines and Cerami, 2005; Juul, 2004; Sirén et al., 2009). Importantly, many recent human studies in schizophrenia and multiple sclerosis convincingly demonstrated EPO effects on cognition (Ehrenreich, Fischer et al., 2007; Ehrenreich, Hinze-Selch et al., 2007). Finally, the hematopoiesis-independent effect of EPO on the nervous system has been proven by slight modification of the EPO molecule, rendering it nonhematopoietic but still tissue-protective (Sirén et al., 2009).

Our previous work in healthy young mice treated for 3 weeks with EPO revealed improvement of hippocampal memory. At the time of improved memory, an increase in short-term and long-term potentiation was documented in hippocampal slices (Adamcio et al., 2008). Along the same lines, application of a single high intravenous dose of EPO in healthy human subjects enhanced the hippocampal response during memory retrieval measured by functional magnetic resonance imaging one week later (Miskowiak et al., 2007). However, some preclinical studies using disease models failed to show effects of EPO treatment on cognition in their respective healthy control groups (Mala et al., 2005; Mogensen et al., 2008). This might be explained by the use of tests (e.g. T-maze, 8-arm radial maze), well suited to measure pathology but less sensitive for detecting fine improvements in cognitive performance in healthy individuals.

To further delineate EPO-mediated improvement of cognition in healthy mice, where no disease-associated variables interfere, we aimed in project II at investigating the spectrum of cognitive functions potentially influenced by EPO. Therefore, we 
employed a sensitive assay for sequentially detecting cognitive performance, the Five Choice Serial Reaction Time Task (5CSRTT), originally developed for rats by Carli and colleagues (Carli et al., 1983) and adapted for mice by Humby and co-workers (Humby, 2005). The 5CSRTT has been used to study attention and executive functions in different rodent models of neuropsychiatric diseases, including attention deficit / hyperactivity disorder (ADHD) (Blondeau and Dellu-Hagedorn, 2007), schizophrenia (Lecourtier and Kelly, 2005) and impulsivity disorders (Isles et al., 2004; Robbins, 2002). With 5CSRTT, we systematically investigated the effect of EPO on different types of learning, memory and attention in healthy young mice.

In this study, $45 \mathrm{C} 57 \mathrm{BL} / 6 \mathrm{NCrl}$ male mice (randomly assigned to one of three groups: EPO, placebo or "no-inject", 15 mice/group) were treated for 3 weeks with EPO $(5000 \mathrm{IU} / \mathrm{kg})$ versus placebo intraperitoneally every other day, beginning at postnatal day 28. After termination of treatment, mice were started on the 5CSRTT, with daily training and testing extending to about 3 months. Overall, a significantly higher proportion of EPO treated mice finished the task, i.e. reached the criteria of adequately reacting to a $1.0 \mathrm{sec}$ flash light out of five arbitrarily appearing choices. During acquisition of this capability, i.e. over almost all sequential training phases, learning readouts (magazine training, operant and discriminant learning, stability of performance) were superior in EPO versus control mice. 


\subsection{ORIGINAL PUBLICATION}

El-Kordi A, Radyushkin K and Ehrenreich H (2009). Erythropoietin improves operant conditioning and stability of performance in mice. BMC Biology, 7:37.

Personal contribution

I was involved in the design of the study, conduction of all behavioral experiments, analysis and interpretation of behavioral data and preparation of the manuscript. 


\title{
Erythropoietin improves operant conditioning and stability of cognitive performance in mice
} Ahmed El-Kordi ${ }^{\dagger}$, Konstantin Radyushkin ${ }^{\dagger}$ and Hannelore Ehrenreich*

Address: Division of Clinical Neuroscience, Max Planck Institute of Experimental Medicine, Göttingen, Germany

Email: Ahmed El-Kordi - kordi@em.mpg.de; Konstantin Radyushkin - radyushkin@em.mpg.de; Hannelore Ehrenreich* - ehrenreich@em.mpg.de

* Corresponding author †Equal contributors

Published: 8 July 2009

BMC Biology 2009, 7:37 doi:10.1/86/174|-7007-7-37
Received: 6 February 2009

Accepted: 8 July 2009

This article is available from: http://www.biomedcentral.com/174I-7007/7/37

(c) 2009 El-Kordi et al; licensee BioMed Central Ltd.

This is an Open Access article distributed under the terms of the Creative Commons Attribution License (http://creativecommons.org/licenses/by/2.0), which permits unrestricted use, distribution, and reproduction in any medium, provided the original work is properly cited.

\begin{abstract}
Background: Executive functions, learning and attention are imperative facets of cognitive performance, affected in many neuropsychiatric disorders. Recently, we have shown that recombinant human erythropoietin improves cognitive functions in patients with chronic schizophrenia, and that it leads in healthy mice to enhanced hippocampal long-term potentiation, an electrophysiological correlate of learning and memory. To create an experimental basis for further mechanistic insight into erythropoietin-modulated cognitive processes, we employed the Five Choice Serial Reaction Time Task. This procedure allows the study of the effects of erythropoietin on discrete processes of learning and attention in a sequential fashion.
\end{abstract}

Results: Male mice were treated for 3 weeks with erythropoietin $(5,000 \mathrm{lU} / \mathrm{kg})$ versus placebo intraperitoneally every other day, beginning at postnatal day 28. After termination of treatment, mice were started on the Five Choice Serial Reaction Time Task, with daily training and testing extending to about 3 months.

Overall, a significantly higher proportion of erythropoietin-treated mice finished the task, that is, reached the criteria of adequately reacting to a $1.0 \mathrm{sec}$ flash light out of five arbitrarily appearing choices. During acquisition of this capability, that is, over almost all sequential training phases, learning readouts (magazine training, operant and discriminant learning, stability of performance) were superior in erythropoietin-treated versus control mice.

Conclusion: Early erythropoietin treatment leads to lasting improvement of cognitive performance in healthy mice. This finding should be exploited in novel treatment strategies for brain diseases.

\section{Background}

The haematopoietic growth factor erythropoietin (EPO) has been in clinical use for over 20 years to treat patients with anaemic conditions, ranging from renal failure to cancer. Upon introduction of EPO to the clinic, it was observed that cognitive performance of treated individuals also improved. This improvement was essentially attributed to anaemia correction with subsequently enhanced tissue oxygenation also in the brain [1]. Much later, EPO and its receptor were found to be produced by 
and act on cells of the nervous system [2,3]. Many reports on neuroprotective and neuroregenerative effects of EPO in rodent models of neurological diseases followed [4] (for review see $[5,6]$ ). Our recent human studies in schizophrenia and multiple sclerosis revealed profound EPO effects on cognitive performance $[7,8]$. The separation of haematopoietic and neuroprotective properties upon slight modification of the EPO molecule ultimately proved the haematopoiesis-independent effect of EPO on the nervous system [6].

To investigate, in the absence of interfering disease variables, the physiological role of the brain EPO system regarding cognition, we performed a series of studies in healthy young mice where we found hippocampal memory, measured by classical fear conditioning, improved after 3 weeks of EPO treatment. At the time of improved memory, an increase in short-term and long-term potentiation was documented in hippocampal slices [9]. Along the same lines, application of a single high intravenous dose of EPO in healthy human volunteers enhanced the hippocampal response during memory retrieval measured by functional magnetic resonance imaging one week later [10]. It should, however, be also mentioned that preclinical studies using disease models failed to show effects of EPO treatment on cognition in their respective healthy control groups (for example, $[11,12])$. This is most likely explained by the application of tests (for example, Tmaze, 8-arm radial maze), well suited to measure pathology but less sensitive for assessing subtle improvements in cognitive performance in healthy individuals.

Since the development of new therapies, targeting cognitive performance is of major interest for clinical neuroscience, and EPO might here be a promising candidate; thus the spectrum of cognitive functions potentially influenced by EPO has to be better defined. For this purpose, we employed the Five Choice Serial Reaction Time Task (5CSRTT), originally developed for rats by Carli and colleagues [13] and adapted for mice by Humby and coworkers [14]. The 5CSRTT has been used to analyse attention and executive functions in different rodent models of neuropsychiatric diseases, including attention-deficit/ hyperactivity disorder [15], schizophrenia [16] and impulsivity disorders [17] (for review, see [18]). With 5CSRTT, we systematically studied the effect of EPO on different types of learning, memory and attention in healthy young mice. We note that obtaining cognitive results in healthy young individuals cannot automatically be translated to disease situations or aged mice. Nevertheless, this approach is the simplest first step to providing a foundation for mechanistic insight. We report here that early EPO treatment indeed improves most of the sequential learning and memory components of a complex longterm cognitive task, ultimately leading to better and more stable cognitive achievements.

\section{Results}

Erythropoietin increases overall performance in 5CSRTT First, we compared the overall performance of EPO, placebo, or no-inject groups in 5CSRTT. Kaplan-Meier analysis revealed that the proportion of mice that finished training in the 5CSRTT (that is, reached criteria of stably responding to a 1.0 -sec stimulus duration on three consecutive days) up to day 94 after cessation of treatment was significantly higher in the EPO-treated group as compared with placebo and no-inject groups (Log-Rank test, $P$ $=0.02)$. There were no differences between placebo and no-inject groups (Figure 1). Taken as a group, EPO-treated mice learn faster.

\section{Erythropoietin accelerates associative, operant and discriminant learning in 5CSRTT}

Since different types of learning determine performance in the 5CSRTT, we asked whether the superiority of EPOtreated mice is reflected in initial learning parameters. Indeed, the number of head entries during habituation and magazine training appeared higher throughout all phases (M1-M4) in EPO-treated mice compared with the

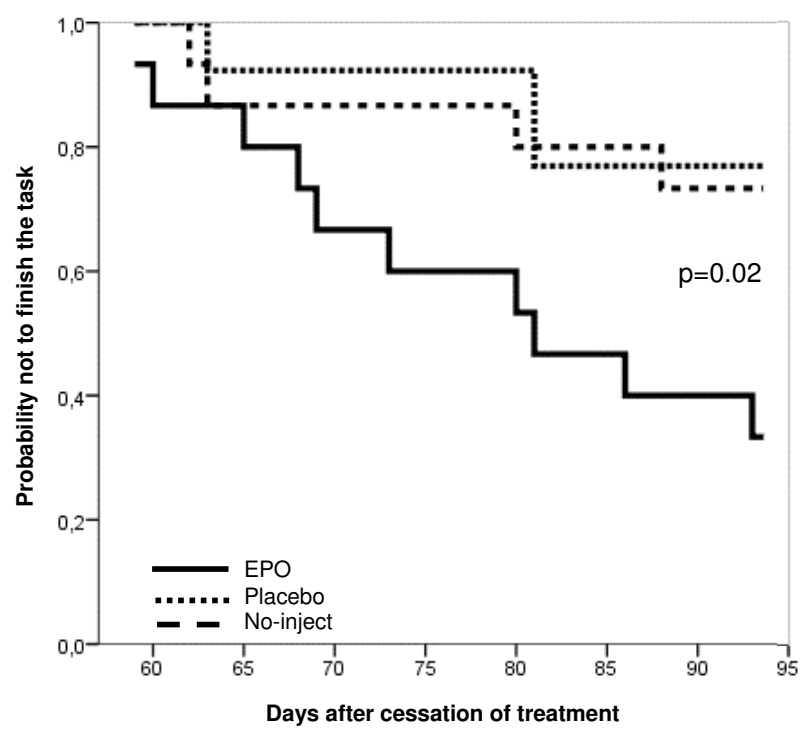

\section{Figure I}

Kaplan-Meier presentation of overall group performance in mice. Curves represent group performance $(n=$ 13-I5 per group). They indicate the cumulative probability of members of each group not to finish the task (that is, not to reach performance criteria in the $1.0-\mathrm{sec}$ stimulus duration phase). EPO-treated mice are superior, that is, have a lower probability not to finish the task as compared with no-inject and placebo groups. On day 59 after cessation of treatment, the first mouse reached criteria in the 1.0 -sec phase. This mouse belonged to the EPO group. Day 94 is the time point on which $>60 \%$ of one experimental group (here the EPO group) had finished the task. 
placebo group (Figure 2A). A significant difference between groups in phases M1 and M4 of magazine training by Kruskal-Wallis test $(P<0.05$ for each) could be attributed to superior performance of the EPO versus placebo group following post hoc analysis (Dunn's multiple comparison test, $P<0.01$ ). During the operant learning phase (S1), EPO-treated mice made more nose pokes (ANOVA: $F_{(1,41)}=8.81, P=0.005$ ) on days 2 and 3 (post hoc $P<0.001$ and $P<0.01$, respectively) (Figure $2 \mathrm{~B}$ ). Additionally, there was a significant group effect in the discriminant learning phase (S2), with EPO-treated mice demonstrating higher choice accuracy (ANOVA: $F_{(1,41)}=$ 5.35, $P=0.026$ ) (Figure 2C).

We wondered whether the improvement in operant and discriminant learning parameters would continue in face of more complex conditions and thus enhanced cognitive challenge during the 5CSRTT training. In fact, the 5CSRTT procedure is much more complex than the preceding shaping phases and consists of more parallel stimuli in addition to stimulus duration (for example, number of stimulus lights, more restricted time-out period). We analysed accuracy in the consecutive stimulus duration phases: $16 \mathrm{sec}, 8 \mathrm{sec}$ and $4 \mathrm{sec}$. The percentage of correct responses over the first 3 days of each phase served as readout of initial learning capabilities in the 5CSRTT training (Figure 2D, E and 2F). EPO-treated mice performed better in 16-sec and 8-sec phases (ANOVA $F_{(1,41)}=7.21 ; P=0.01$ and $F_{(1,39)}=4.87 ; P=0.03$, respectively, Figure $2 \mathrm{D}$ and $2 \mathrm{E}$ ), but no longer in the following ones, from $4 \mathrm{sec}$ (Figure $2 \mathrm{~F}$ ) to $2 \mathrm{sec}, 1.8 \mathrm{sec}, 1.4 \mathrm{sec}, 1.2 \mathrm{sec}$ and $1.0 \mathrm{sec}$ (data not shown). Overall accuracy increased over consecutive phases until reaching a plateau at $4 \mathrm{sec}$ for both groups, which then stayed essentially stable over the following
A Magazine training

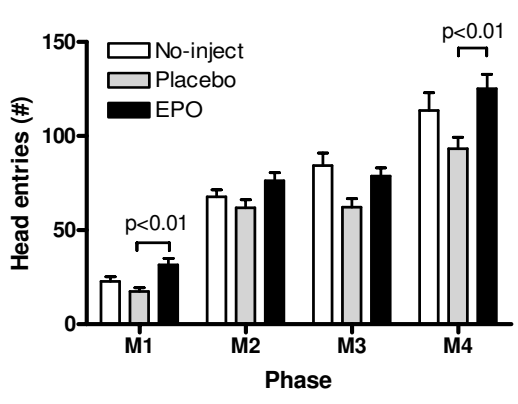

D Stimulus duration $16 \mathrm{sec}$

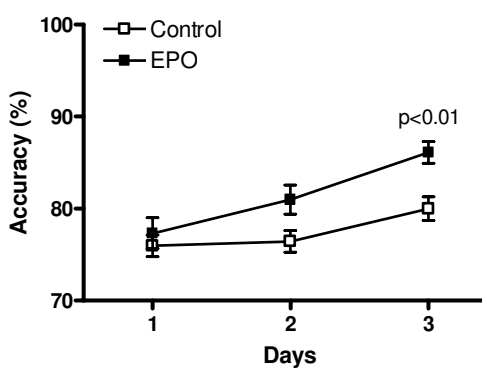

B Operant learning (S1)

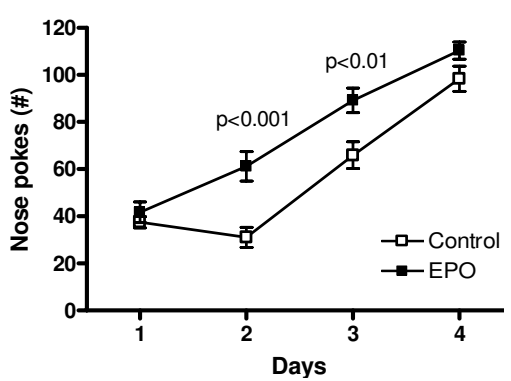

Initial 5CSRTT training phases

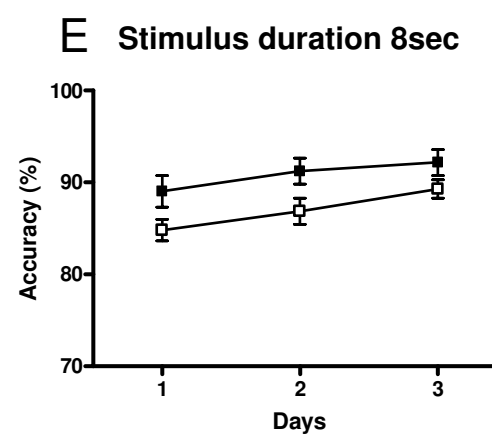

C Discriminant learning (S2)
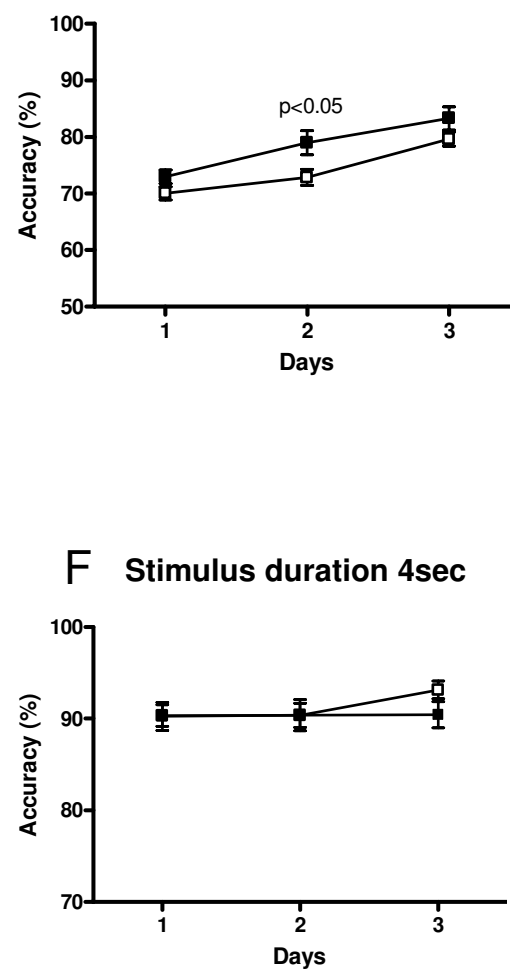

Figure 2

Analysis of distinct sequential learning phases. (A) Number of head entries as indicators of associative learning in magazine training (MI-4) show an overall significant effect of EPO treatment compared with placebo. (B) Number of nose pokes in the operant learning phase (SI) as well as (C) percentage of correct nose pokes in the discriminant learning phase (S2) were higher in the EPO-treated compared with the control group (placebo plus no-inject). (D, E) Initial cognitive performance in the 5CSRTT was better upon EPO treatment in the 16-sec and 8-sec stimulus duration phases, but no longer in the 4-sec phase (F); $n=14-28$ per group; data presented as mean \pm S.E.M.; all significance values refer to post hoc tests which were only significant if ANOVA was also significant; in $(E)$, only ANOVA was significant $(P=0.03)$. 
shorter-duration phases (data not shown). There was no consistent difference in accuracy (nor amount of omissions) during the attentional challenge phase of $0.8 \mathrm{sec}$ $\left(F_{(1,18)}=0.49, P=0.5\right.$ and $F_{(1,18)}=1.45, P=0.24$, respectively).

\section{Erythropoietin improves task adaptation and stabilizes performance in 5CSRTT}

We next analysed the number of training days required to reach performance criteria in 5CSRTT for each stimulus duration, that is, acquisition days (Figure 3A). There was a significant effect of stimulus duration on the number of training days needed, independent of treatment $\left(F_{(7,126)}=\right.$ $8.24, P<0.0001)$. Starting immediately from 2 sec, mice required more days to reach criteria compared with previous phases. To further address the phenomenon of an abrupt increase of training days in the 2-sec phase (independently of treatment), we analysed omissions more closely. In fact, omissions in all training phases from 16 sec to $1 \mathrm{sec}$ were comparable between the two experimental groups, and rates were low up to the switch from the 4sec to the 2-sec phase (for illustration, data of the respective first training day of each stimulus duration are given as an example: range of omissions on day 1 of 16 sec: 6.55 \pm 4.28 EPO versus $5.83 \pm 4.17$ control; on day 1 of 8 sec: $9.45 \pm 13.09$ EPO versus $8.93 \pm 8.99$ control; on day 1 of 4 sec: $16.90 \pm 9.94$ EPO versus $16.90 \pm 9.81$ control; on day 1 of 2 sec: $29.78 \pm 12.86$ EPO versus $35.89 \pm 10.27$ control; on day 1 of $1.8 \mathrm{sec} 22.11 \pm 7.46$ EPO versus 21.96 \pm 8.75 control; on day 1 of $1.4 \mathrm{sec}: 27.00 \pm 7.58$ EPO versus $28.93 \pm 11.12$ control; on day 1 of $1.2 \mathrm{sec}$ : $24.67 \pm$ 8.21 EPO versus $24.1 \pm 8.77$ control; on day 1 of $1.0 \mathrm{sec}$ :
$24.7 \pm 5.72 \mathrm{EPO}$ versus $29.06 \pm 10.29$ control). It turned out that from the 4-sec phase to the 2-sec phase, omissions promptly doubled and stayed at a high level up to the end of 5CSRTT training ( $1 \mathrm{sec}$ ), independent of treatment group. This may explain the prominent increase in training day requirement starting from $2 \mathrm{sec}$.

We next averaged omitted trials in each of the six 10-trial blocks over the first 10 days of this 2-sec phase (Figure 3B). There was no significant overall effect of treatment; however, there was a significant interaction between trial block and treatment $\left(F_{(5,130)}=2.37, P=0.04\right)$. Post hoc analysis revealed that EPO-treated mice had significantly fewer omissions in the first trial block compared with controls $(P<0.05)$, pointing to a faster adaptation to the task.

As fluctuations in performance may increase the number of days needed for reaching performance criteria, we analysed the magnitude of fluctuation ('relapsing back from already achieved criteria') in the 2-sec phase. Here, the control group had significantly more relapses compared with the EPO group, pointing to more stable performance upon EPO ( $\mathrm{Chi}^{2}$ test, $P=0.04$ ) (Figure 3C). To further explore the effect of EPO on stability of performance, we analysed total omissions after reaching criteria (1.0 sec) over additional 4 days. EPO-treated mice were superior $(P$ $=0.04$ ) as compared with the control group (Figure $3 \mathrm{D}$ ).

\section{Erythropoietin does not affect locomotor activity in 5CSRTT}

To clarify whether increased locomotion has contributed to the superior performance of EPO-treated mice in
A Acquisition days

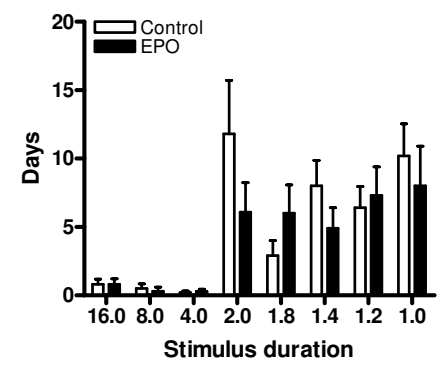

B First ten days in 2 sec phase

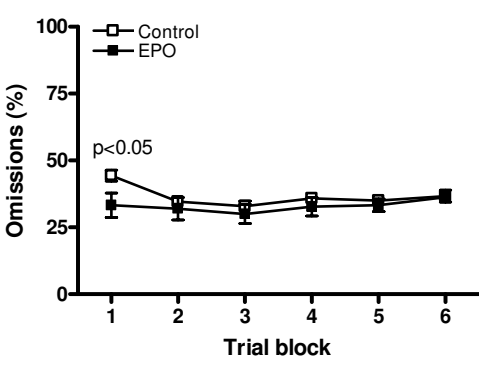

\section{Relapse analysis at 2 sec phase}

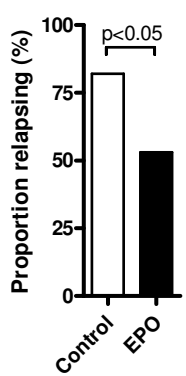

Total omissions in $1.0 \mathrm{sec}$ phase

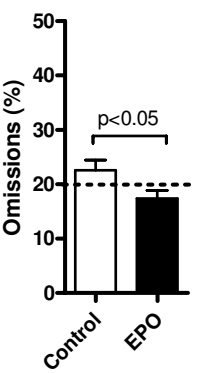

Figure 3

Analysis of selected readouts of cognitive performance in high-performer mice. (A) Number of acquisition days required for reaching performance criteria at each stimulus duration phase shows no group differences but a sharp increase in the transition from $4 \mathrm{sec}$ to $2 \mathrm{sec}$. (B) The EPO-treated group showed faster task adaptation: The proportion of omissions in the first of six 10-trial blocks collapsed across the first 10 days of the 2-sec phase was significantly lower. Post hoc test. (C) The EPO-treated group showed higher stability of performance: the proportion of mice that relapsed from already reached performance criteria in the $2-\mathrm{sec}$ stimulus duration phase was smaller. Chi ${ }^{2}$ test. (D) EPO improves performance stability even in the I-sec phase: Proportion of total omissions over 4 days after reaching performance criteria was significantly lower in the EPO group. Mann-Whitney test. $N=7-28$; data presented as mean \pm S.E.M. 
3 Weeks of injection

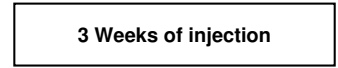

Habituation and magazine training
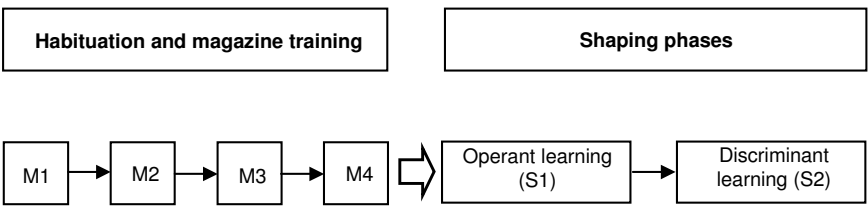

(S1)

$\rightarrow$
learning (S2)
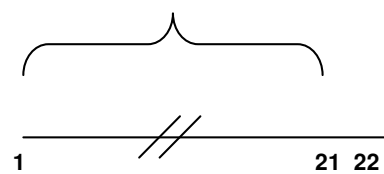

2122

Figure 4

Experimental design. Following 3 weeks of EPO (5,000 IU/kg body weight intraperitoneal) versus placebo (diluent control) treatment or handling only (no-inject group), 7-week-old mice go through habituation/magazine training (MI-M4) and shaping phases (SI-S2) before starting training in the 5CSRTT. Here they move from $16 \mathrm{sec}$ to the respective next phase with lower stimulus duration ( $8 \mathrm{sec}$ and so on) upon reaching fixed criteria. Having reached criteria in the I.0-sec phase, their attention is challenged by further shortening stimulus duration $(0.8 \mathrm{sec})$.

phases with 16-sec and 8-sec stimulus duration, we analysed the average of reward latency (that is, the time between responding correctly and collecting reward) as well as the 'latency correct' (that is, the time between stimulus presentation and responding correctly) in the corresponding phases. There were no differences between groups in either reward latency or in 'latency correct' in any phase of the 5CSRTT training (data not shown).

\section{Discussion}

In the present study, a 3-week high-dose EPO treatment of healthy young mice increased the probability of these animals finishing training in the 5CSRTT. While there were no differences in the number of total days needed to terminate the task among ultimately successful individual mice, regardless of group assignment, the proportion per group was different, with more successful mice in the EPO-treated cohort. EPO-treated mice showed superior performance in associative, operant and discriminant learning as well as in initial 5CSRTT training phases. Moreover, EPO-treated mice demonstrated better task adaptation and higher performance stability. In contrast, with the number of mice remaining in the task, there were no clear effects with this particular EPO treatment schedule (terminated more than 3 months before) on attentional performance, as defined by response to the 0.8 -sec stimulus duration.

To gain an overall impression of the progress in cognitive training of our mouse groups, we employed the survival analysis of Kaplan-Meier. This methodological approach was crucial for our purposes as it describes, over a long testing period, the development of group performance in a higher cognitive task, the 5CSRTT. By applying standard statistical methods only, the clear superiority of the EPO group would not have been detectable. In fact, the KaplanMeier results inspired us to dissect out potential learning processes contributing to the superior group performance of EPO-treated mice. The success of this statistical approach would suggest the usefulness of this method for future group analysis in the 5CSRTT.

While clear benefits of EPO treatment could easily be demonstrated throughout all initial learning phases including the 5CSRTT training up to 8-sec stimulus duration, we did not find differences in accuracy in phases with stimulus durations of $4 \mathrm{sec}$ and below. This might be due to a ceiling effect, as both groups had reached almost maximal performance at this stage. Nevertheless, also in these more progressed phases, EPO superiority was visible when analysing more subtle readouts of cognitive performance, for example, stability and task adaptation.

A particularly difficult 5CSRTT training step for mice is the switching from the 4-sec to the 2-sec stimulus duration phase. In this challenging phase, EPO-treated mice had consistently lower omission rates when entering the task, indicating immediate task adaptation. This improvement just failed to translate into significant differences in acquisition days. Interestingly, a previous study on galanin transgenic mice, a model for impaired learning capacity, reported for this critical 2 -sec phase more than doubling of required training days compared with controls, due to more omissions [19]. In contrast to the improved task adaptation shown in the present study, reflected by less omissions in the first trial block, galanin transgenic mice exhibited more omissions in the last two trial blocks, pointing to deficiency of sustained attention [19]. In contrast to all other studies employing 5CSRTT, we have additionally analysed parameters representing stability of 
performance. The analysis of relapse frequency (that is, dropping down from a once-acquired performance level) in the challenging 2-sec stimulus duration phase with its increasing attentional challenge again revealed a more consistent performance by the EPO group.

An important limitation of the current study (and of 5CSRTT in general) has to be addressed: the strong selection bias of mice with higher cognitive abilities means that mice that do not learn crucial steps of the task are excluded from further testing (which is frequently not mentioned in respective publications). This gradual decrease of numbers in advanced training phases renders statistical analysis increasingly difficult. For instance, in the current study, there would not have been enough mice in the placebo and the no-inject group for separate analysis in the attentional phase with stimulus duration of 0.8 $\mathrm{sec}$, despite starting out with 15 mice per group. A total of 45 mice to be run simultaneously in this task already reaches the limits of a generous set-up of five chambers and more than a full working day of the investigator. Since performance of placebo and no-inject groups did not differ much in the early phases, pooling of both groups to obtain a 'control cohort' was possible.

\section{Conclusion}

The findings of the present study, that is, improved sequential learning and memory components of a complex long-term cognitive task upon EPO treatment, will provide the basis for further work targeting molecular facets of these critical phases. Such studies will include quantitative and qualitative evaluation of neurogenesis and synapse formation in respective brain regions, for example, cingulate cortex and hippocampus. In the latter, we recently detected increased long-term potentiation after EPO treatment [9]. In addition, future work will have to assess whether the effects of EPO obtained here are restricted to the use of very young and healthy animals, or would be similarly strong in older mice and/or disease conditions. Further untangling of molecular mechanisms of EPO action on higher cognitive functions may then ultimately open new avenues for prevention strategies and therapeutic interventions in neuropsychiatric diseases.

\section{Methods}

\section{Animals}

Forty-five male C57BL/6NCrl mice (Charles River, Sulzfeld, Germany), 3 weeks old upon arrival, were housed in groups of five in standard plastic cages, with food and water ad libitum. The temperature in the colony room was maintained at $20-22^{\circ} \mathrm{C}$, the light-dark cycle at $12 \mathrm{~h}$ (light on at $0400 \mathrm{hrs}$ ). After 7 days of acclimatising to the new environment, injections were started at the age of 28 days and were always performed in the first half of the light phase. Behavioural experiments were conducted by an investigator, blinded to treatment condition, during the second half of the light phase (between $1000 \mathrm{hrs}$ and $1500 \mathrm{hrs}$ ). All experiments were approved by the local Animal Care and Use Committee in accordance with the German Animal Protection Law.

\section{Injection protocol}

Mice were randomly assigned to one of three groups: EPO, placebo or 'no-inject' (to uncover potential effects of repeated injection stress), each consisting of 15 mice. Mice were intraperitoneally injected every other day for 21 days (11 injections in total) either with EPO (5,000 IU/kg, Epoetin-alpha, Janssen-Cilag, Neuss, Germany) or with placebo (diluent buffer) in a volume of $0.01 \mathrm{ml} / \mathrm{g}$ body weight. Mice from the no-inject group were just weighed every other day (in order to keep handling similar to the injected mice) with no additional manipulations. Training started 1 day after cessation of injections.

\section{CSRTT apparatus}

Mice were trained in an operant chamber (height $18 \mathrm{~cm}$, width $15.5 \mathrm{~cm}$, depth $20 \mathrm{~cm}$, Med Associates Inc, St. Albans, USA), enclosed in a sound-attenuating box and connected to a Fujitsu Siemens PC. One wall of the operant chamber had a curved shape and carried an array of five stimulus holes. The stimulus holes were $1.2 \mathrm{~cm}$ in diameter and contained an LED stimulus light (depth 1 $\mathrm{cm}$ ) in the rear. Infrared photocell pairs were located at 4 $\mathrm{mm}$ from the entrance of the stimulus holes and detected nose pokes of mice into the holes. The wall opposite to the stimulus holes contained a magazine cup, also with a photocell detector of head entries, in which liquid reward (4\% sucrose solution) was delivered always simultaneously with illumination of the magazine. The house light was located $32 \mathrm{~cm}$ above the magazine.

\section{Habituation and magazine training}

Two days before starting training, mice were habituated to the liquid reward of $4 \%$ sucrose solution in their home cages overnight. The day before starting magazine training, sucrose bottles were removed and mice were waterdeprived. Water deprivation was applied during the whole experimental period. Immediately after finishing the daily test sessions, mice were given water in individual cages for $20 \mathrm{~min}$. Magazine training consisted of four consecutive phases (M1-M4), one phase per day, each lasting for 15 min, with all stimulus holes closed. In the first phase (M1), liquid reward was delivered ( $10 \mu \mathrm{l})$ upon initiation of the training session. In the second phase of magazine training (M2), the number of potential rewards was increased, with a fixed interval of $118 \mathrm{sec}$ between reward presentations. A head entry into the magazine was required to collect the reward. In the third phase (M3), the fixed interval was replaced by a head entry-dependent interval of $100 \mathrm{sec}$ to obtain reward. In the last phase 
(M4), this interval was further reduced to $50 \mathrm{sec}$, ideally yielding a consistently increasing number of head entries. Head entries into the magazine together with reward consumption were taken as indicators for associating the magazine with reward delivery (see experimental design Figure 4).

\section{Shaping phases (operant and discriminant learning)}

During shaping, mice were trained to perform a nose poke into an illuminated stimulus hole in order to obtain reward. The shaping procedure consisted of two phases (each extending over several days, dependent on individual performance, and with a daily session duration of 30 min) where mice were taught to associate nose poking into an illuminated hole with reward (phase S1), and then trained to discriminate between those nose pokes that lead to reward (illuminated holes) and those that do not (unlit holes) (phase S2) (Figure 4). Throughout shaping all stimulus holes were open. During S1, all stimulus lights were on. Any nose poke in a stimulus hole was rewarded. The inter-trial interval (time from pick-up of reward to next stimulus hole illumination) was set to 8 sec. During S2, presentation of lit and unlit holes was conducted in a pseudorandom manner. Mice were only rewarded upon nose poking into a lit stimulus hole. Performing a nose poke in an unlit hole led to switch-off of the house light for $5 \mathrm{sec}$. Mice in S1 were moved to the next training phase once they had reached 35-40 nose pokes each on three consecutive days. S2 was terminated when mice had arrived at a stable performance of $\geq 70 \%$ correct responses. Starting from analysis of shaping phases, placebo and no-inject groups were pooled, since they no longer differed in any of the high-performing tasks beyond habituation.

\section{CSRTT training}

The training session started with illumination of magazine light and presentation of $4 \%$ sucrose solution. Head entry started the trial. At $8 \mathrm{sec}$ after head entry, light (initially set to $16 \mathrm{sec}$ ) was randomly presented in one of the five stimulus holes. A correct response, that is, nose poking into the lit hole, led to reward $(6 \mu \mathrm{l})$ and next trial start after $8 \mathrm{sec}$. Nose poking in an unlit stimulus hole, that is, an incorrect response, led to extinguishing the house light for $5 \mathrm{sec}$ (time-out) and no reward. Further nose pokes during time-out extended that period for additional $5 \mathrm{sec}$ each. If a mouse did not respond by nose poking into any of the holes during stimulus presentation, an omission was counted. As a consequence, no reward was presented. Also omissions provoked time-out. A training session was terminated after $30 \mathrm{~min}$ or upon performing 60 trials, whichever came first. Mice were trained in the phase with 16 -sec stimulus duration until they reached clearly defined performance criteria $(\geq 75 \%$ accuracy (correct responses/correct + incorrect responses $* 100), \leq 20 \%$ omissions and at least 50 trials performed over three consecutive days). Eight such phases followed with gradually declining stimulus duration up to $1.0 \sec (16,8,4,2,1.8$, $1.4,1.2$, and $1.0 \mathrm{sec}$ ).

In the first phases, mice had time to respond as long as the stimulus light was on. For phases with stimulus duration below $5 \mathrm{sec}$, the response time (so-called limited hold) was added up to $5 \mathrm{sec}$. Having 'finished the task' meant having reached the above performance criteria for the 1.0 sec phase. Training sessions were performed every day, including weekends. As soon as $>60 \%$ of the mice in one of the three experimental groups had finished the task by reaching performance criteria in the $1.0 \mathrm{sec}$ phase, regular 5CSRTT training was stopped for all groups (day 94; Figure 1) and only high performers of all groups were carried on with attention testing, that is, stimulus duration below $1.0 \mathrm{sec}(0.8 \mathrm{sec})$. This resulted in EPO $n=10$ versus control $n=10$ (placebo $n=4$ plus no-inject $n=6$ ).

\section{Overview on parameters of task acquisition}

The following parameters were employed: (1) proportion of mice that finished the task in all experimental groups; (2) number of days until reaching performance criteria in all phases (acquisition days); (3) proportion of omissions, that is, number of omitted per total number of performed trials within one training session; (4) number of head entries during a magazine training session; (5) number of nose pokes during an operant learning session; (6) accuracy, that is, percentage of correct responses calculated as number of correct responses/correct + incorrect responses*100; (7) sustained attention, that is, attentional performance, expressed as omissions as a function of time in session, evaluated over the first 10 days in the 2 -sec phase (the most challenging training step). Data are expressed in 10-trial blocks collapsed across all 10 days; (8) newly introduced parameter: Stability of performance/ relapses, that is, the ability to maintain a high performance with respect to omissions and accuracy for three consecutive days. Mice that reached the above mentioned criteria without keeping them for the required 3 days were considered relapsing. An additional measure of performance stability in high performers were omissions in the 1.0-sec stimulus duration phase, as determined for 4 days after reaching criteria.

\section{Statistical analysis}

Statistical analysis was performed using the statistical programs SPSS for windows, release 16 (SPSS Inc., Chicago, USA) and GraphPad Prism version 4.00 for Windows, (GraphPad Software, San Diego, USA). We applied twoway ANOVA repeated measures, Kruskal-Wallis test, chi²test and Mann-Whitney test where indicated. Bonferroni and Dunn's multiple comparison tests were used for post hoc analysis. Threshold for significance was $P<0.05$. Sur- 
vival analysis/Kaplan-Meier curves [20] were introduced to demonstrate the proportion of mice per group finishing the 5CSRTT training up to the 1.0-sec stimulus duration (task goal). Mice that did not reach the goal until day 94 were censored.

\section{Authors' contributions}

AEK and KR carried out the behavioural experiments. AEK performed statistical analysis of experimental results. KR participated in the design of the study and in writing of the manuscript. AEK and HE wrote the manuscript. HE supervised the whole project and designed the study. All authors read and approved the final manuscript.

\section{Acknowledgements}

We would like to express our gratitude to Anja Ronnenberg for her help in the behavioural testing. This study has been supported by the MaxPlanck-Society, the DFG Centre for Molecular Physiology of the Brain (CMPB) as well as by private donations.

\section{References}

I. Jelkmann W: Effects of erythropoietin on brain function. Curr Pharm Biotechnol 2005, 6(I):65-79.

2. Masuda S, Nagao M, Takahata K, Konishi Y, Gallyas F Jr, Tabira T, Sasaki R: Functional erythropoietin receptor of the cells with neural characteristics. Comparison with receptor properties of erythroid cells. J Biol Chem I993, 268(I5): I | 208-I I2I6.

3. Masuda S, Okano M, Yamagishi K, Nagao M, Ueda M, Sasaki R: A novel site of erythropoietin production. Oxygen-dependent production in cultured rat astrocytes. I Biol Chem 1994, 269(30): 19488-19493.

4. Juul S: Recombinant erythropoietin as a neuroprotective treatment: in vitro and in vivo models. Clin Perinatol 2004, 3I(I): I29-I42.

5. Brines M, Cerami A: Emerging biological roles for erythropoietin in the nervous system. Nat Rev Neurosci 2005, 6(6):484-494.

6. Siren AL, Fasshauer T, Bartels $C$, Ehrenreich $\mathrm{H}$ : Therapeutic Potential of Erythropoietin and its Structural or Functional Variants in the Nervous System. Neurotherapeutics 2009, 6(I): 108-127.

7. Ehrenreich H, Fischer B, Norra C, Schellenberger F, Stender N, Stiefel M, Siren AL, Paulus W, Nave KA, Gold R, Bartels C: Exploring recombinant human erythropoietin in chronic progressive multiple sclerosis. Brain 2007, I30(Pt 10):2577-2588.

8. Ehrenreich $\mathrm{H}$, Hinze-Selch D, Stawicki S, Aust C, Knolle-Veentjer $S$, Wilms S, Heinz G, Erdag S, Jahn H, Degner D, Ritzen M, Mohr A, Wagner M, Schneider U, Bohn M, Huber M, Czernik A, Pollmacher T, Maier W, Siren AL, Klosterkotter J, Falkai P, Ruther E, Aldenhoff JB, Krampe $\mathrm{H}$ : Improvement of cognitive functions in chronic schizophrenic patients by recombinant human erythropoietin. Mol Psychiatry 2007, I 2(2):206-220.

9. Adamcio B, Sargin D, Stradomska A, Medrihan L, Gertler C, Theis F, Zhang M, Muller M, Hassouna I, Hannke K, Sperling S, Radyushkin K, El-Kordi A, Schulze L, Ronnenberg A, Wolf F, Brose N, Rhee JS, Zhang W, Ehrenreich $\mathrm{H}$ : Erythropoietin enhances hippocampal long-term potentiation and memory. BMC Biol 2008, 6:37.

10. Miskowiak K, O'Sullivan U, Harmer CJ: Erythropoietin enhances hippocampal response during memory retrieval in humans. Neurosci 2007, 27(I I):2788-2792.

II. Mala H, Alsina CG, Madsen KS, Sibbesen EC, Stick H, Mogensen J: Erythropoietin improves place learning in an 8-arm radial maze in fimbria-fornix transected rats. Neural Plast 2005, I 2(4):329-340.

12. Mogensen J, Boyd MH, Nielsen MD, Kristensen RS, Mala H: Erythropoietin improves spatial delayed alternation in a T-maze in rats subjected to ablation of the prefrontal cortex. Brain Res Bull 2008, 77(I): I-7.

13. Carli M, Robbins TW, Evenden JL, Everitt BJ: Effects of lesions to ascending noradrenergic neurones on performance of a 5- choice serial reaction task in rats; implications for theories of dorsal noradrenergic bundle function based on selective attention and arousal. Behav Brain Res 1983, 9(3):36I-380.

14. Humby T, Wilkinson L, Dawson G: Assaying aspects of attention and impulse control in mice using the 5 -choice serial reaction time task. Curr Protoc Neurosci 2005, Chapter 8:Unit 8.5H.

15. Blondeau C, Dellu-Hagedorn F: Dimensional analysis of ADHD subtypes in rats. Biol Psychiatry 2007, 6 I (1 2): 1340-1350.

16. Lecourtier L, Kelly PH: Bilateral lesions of the habenula induce attentional disturbances in rats. Neuropsychopharmacology 2005, 30(3):484-496.

17. Isles AR, Humby T, Walters E, Wilkinson LS: Common genetic effects on variation in impulsivity and activity in mice. J Neurosci 2004, 24(30):6733-6740.

18. Robbins TW: The 5-choice serial reaction time task: behavioural pharmacology and functional neurochemistry. Psychopharmacology (Berl) 2002, 163(3-4):362-380.

19. Wrenn CC, Turchi JN, Schlosser S, Dreiling JL, Stephenson DA, Crawley JN: Performance of galanin transgenic mice in the 5choice serial reaction time attentional task. Pharmacol Biochem Behav 2006, 83(3):428-440.

20. Kleinbaum D: Survival analysis: A Self-Learning Text New York: Springer; 1996.
Publish with Biomed Central and every scientist can read your work free of charge

"BioMed Central will be the most significant development for disseminating the results of biomedical research in our lifetime. "

Sir Paul Nurse, Cancer Research UK

Your research papers will be:

- available free of charge to the entire biomedical community

- peer reviewed and published immediately upon acceptance

- cited in PubMed and archived on PubMed Central

- yours - you keep the copyright

Submit your manuscript here:

http://www.biomedcentral.com/info/publishing_adv.asp 


\section{EFFECT OF OVER-EXPRESSION OF EPOR IN THE FOREBRAIN AND HIPPOCAMPUS OF MICE ON COGNITIVE PERFORMANCE}

\subsection{OVERVIEW OF PROJECT III}

Cognitive deficits are the core of many neuropsychiatric diseases. It has been shown in project II and in other studies that EPO improves cognitive performance in health and disease (Adamcio et al., 2008; Ehrenreich, Fischer et al., 2007; Ehrenreich, Hinze-Selch et al., 2007; El-Kordi, Radyushkin and Ehrenreich, 2009). In project III we aimed at gaining more mechanistic insight into the cellular contribution to these EPO effects on higher cognition in mice. For this purpose, we chose to target a particular subpopulation of neurons known to be involved in cognition. We developed a transgenic mouse model expressing EPO receptor (EPOR) under the $\alpha$ calcium/calmodulin-dependent protein kinase II ( $\alpha$-CaMKII) promoter, i.e. in pyramidal neurons of cortex and hippocampus. For that, we used a mutant form of the receptor with a point mutation at nucleotide 484 causing a substitution of arginine to cysteine at codon 129 of $\mathrm{N}$ terminus (R129C) leading to disulfide-linked homodimerization of EPOR and rendering it constitutively active. By over-expressing cEPOR, which has been shown to activate EPO-induced signaling without the requirement of its ligand (Fu et al., 2009; Moucadel and Constantinescu, 2005), in principal neurons of cortex and hippocampus, we achieved a reliable and permanent EPO-induced stimulation of a cell type, known to be involved in long-term potentiation and higher brain functions. Wildtype and transgenic mice were analyzed using a wide spectrum of behavioral tasks. Expression of cEPOR in cortical and hippocampal pyramidal neurons of transgenic mice resulted in better performance in Morris water maze compared to their wild type littermates. Moreover, transgenic mice showed increased cognitive flexibility, improved social memory and superior attentional capacities in 5CSRTT. Using electrophysiological techniques, we found that transgenic mice had enhanced synaptic plasticity (long-term potentiation, LTP). However, the superior cognitive performance was accompanied by signs of increased impulsivity under cognitive challenge and mild hyperactivity.

Taken together, selective EPOR activity in pyramidal neurons of hippocampus and cortex prominently affects cognitive performance. These findings will help elucidate the cellular/molecular mechanisms involved in cognitive effects of the brain EPO system and ultimately open new avenues for treatment of neurological and psychiatric diseases. 


\subsection{ORIGINAL PUBLICATION}

Sargin D, El-Kordi A*, Agarwal A, Müller M, Wojcik SM, Hassouna I, Nave K-A and Ehrenreich $\mathrm{H}$ (2010). Expression of constitutively active erythropoietin receptor in cortical neurons boosts higher cognitive functions in mice. Submitted.

*Equally contributing first author

\section{Personal contribution}

I was involved in the design of the behavioral studies, conduction of all behavioral experiments, analysis and interpretation of behavioral data and preparation of the manuscript. Generating the transgenic mice and molecular biological analyses were done by Dr. D. Sargin. Electrophysiological measurements were conducted by our collaborators. 


\title{
Expression of constitutively active erythropoietin receptor in cortical neurons boosts higher cognitive functions in mice
}

\author{
Sargin $D^{1 *}$, El-Kordi $A^{1,2 *}$, Agarwal $A^{3}$, Müller $\mathrm{M}^{2,4}$, Wojcik $S M^{5}$, Hassouna I', \\ Nave $\mathrm{K}-\mathrm{A}^{2,3 \S}$, and Ehrenreich $\mathrm{H}^{1,2 \S}$ \\ ${ }^{1}$ Division of Clinical Neuroscience, Max Planck Institute of Experimental Medicine, \\ 2DFG Research Center for Molecular Physiology of the Brain (CMPB), \\ ${ }^{3}$ Department of Neurogenetics, Max Planck Institute of Experimental Medicine, \\ ${ }^{4}$ Department of Neuro- and Sensory Physiology, Georg-August-University, \\ ${ }^{5}$ Department of Molecular Neurobiology, Max Planck Institute of Experimental Medicine, \\ Göttingen, GERMANY
}

*These authors contributed equally to this work

Running Title: cEPOR in cortical neurons boosts cognition

Word Count Introduction, Results, Discussion: 3.289

Word Count Abstract: 150

Key Words: EPO, neuropsychology, behavior, transgene, 5-CSRTT, impulsivity, neuroplasticity, LTP, hippocampus, neuroprotection

Disclosure: User patents on EPO in schizophrenia and MS have been submitted.

${ }^{\text {s}}$ Correspondence:

Prof. Hannelore Ehrenreich, MD, DVM

Division of Clinical Neuroscience

E-mail: ehrenreich@em.mpg.de

Prof. Klaus-Armin Nave, PhD

Department of Neurogenetics

E-mail: nave@em.mpg.de

Max Planck Institute of Experimental Medicine

Hermann-Rein Str. 3, 37075 Göttingen, Germany

Tel: 49-551-3899-628

Fax: 49-551-3899-670 


\section{ABSTRACT}

Erythropoietin (EPO) and its receptor (EPOR) are expressed in the developing brain and their transcription is upregulated in adult neurons and glia upon injury or neurodegeneration. We have shown neuroprotective effects and improved cognition in patients with neuropsychiatric diseases treated with EPO. However, the critical EPO targets in brain are unknown, and separation of direct and indirect effects has remained difficult, given the role of EPO in hematopoiesis and brain oxygen supply. Here we demonstrate that mice with transgenic expression of a constitutively active EPOR isoform (cEPOR) in pyramidal neurons of cortex and hippocampus exhibit enhancement of spatial learning, cognitive flexibility, social memory, and attentional capacities, accompanied by increased impulsivity. Superior cognitive performance is associated with augmented long-term potentiation of cEPOR expressing neurons in hippocampal slices. That endogenous EPOR activity stimulates plasticity of cortical neurons is unexpected, but functionally complements anti-apoptotic actions of EPO in the recovery from brain injury.

\section{INTRODUCTION}

The hematopoietic growth factor erythropoietin (EPO) expands erythrocytes by preventing apoptosis of erythroblasts in the bone marrow. Recombinant human EPO (rhEPO) is a clinically safe drug, applied for the treatment of anemia worldwide. The surprising clinical observation that rhEPO improves cognitive functions has always been attributed to the increase in hemoglobin levels and thus enhanced tissue oxygenation (e.g. Grimm et al. , 1990 ;Hengemihle et al. , 1996 ;Kramer et al. , 1996 ;Pickett et al. , 1999), (for review, see Ehrenreich et al. , 2008 ;Jelkmann, 2005, 1992). Even after the discovery of EPO and EPO receptor (EPOR) in the brain (Masuda et al. , 1993 ;Masuda et al. , 1994), it took years until direct EPO effects on the central nervous system were first explored by in vivo 
experiments (Sadamoto et al. , 1998). In the following, EPO turned out to have potent antiapoptotic, antioxidative and anti-inflammatory properties in the brain (for review, see e.g. Brines and Cerami, 2005 ;Siren et al. , 2009). Downstream signaling pathways of EPO in cells of the nervous system were extensively explored, showing an involvement of signal transducers and activators of transcription (STATs), phosphatidylinositol-3 kinase (PI3K)/AKT, RAS/extracellular signal-regulated kinase (ERK1/2), nuclear factor kappa B (NF-kappa B) and calcium (Byts et al. , 2008 ;Digicaylioglu and Lipton, 2001 ;Siren et al. , 2001). A large number of preclinical studies followed, devoted to the employment of EPO as a neuroprotective agent (for review, see Sargin et al. , 2010). Recent clinical trials on patients with schizophrenia (Ehrenreich et al. , 2007b ;Wustenberg et al. , 2010) or chronic progressive multiple sclerosis (Ehrenreich et al. , 2007a) as well as a trial involving extremely preterm infants (Neubauer et al. , 2010), which all demonstrated improved cognitive outcome upon EPO treatment, strongly suggested that this growth factor should be considered as a candidate neuroprotective drug counteracting cognitive decline. Nevertheless, the relevant mechanisms of action remained unclear and difficult to formally separate from blood oxygenation effects.

As compared to neuroprotection studies in disease models (for review, see Sargin et al. , 2010), work on the function of the EPO system in normal brain is scarce. Based on the prominent effects of EPO on cognition, we hypothesized that an important physiological role of EPO in postnatal life or adulthood might be the modulation of neuroplasticity and of higher cognitive functions. We showed previously that in healthy young mice high-dose EPO treatment over 3 weeks enhanced hippocampal long-term potentiation (LTP) and memory (Adamcio et al. , 2008), as well as executive and attentional functions (El-Kordi et al. , 2009). A recent functional magnetic resonance imaging ( $\mathrm{fMRI}$ ) study on healthy volunteers reported enhancement of the hippocampal response during memory retrieval after only one high dose of EPO (Miskowiak et al. , 2007). In all of these cases, EPO was applied peripherally, penetrated an intact blood-brain-barrier (Banks et al. , 2004 ;Brines et al. , 2000 ;Ehrenreich et al. , 2004), and likely bound to all major classes of brain cells expressing EPOR, making it impossible to delineate the cell type(s) responsible for enhanced cognition. 
To explore whether EPO-EPOR signaling in cortical neurons has a direct impact on cognitive functions in the non-injured brain, we chose a transgenic strategy. To be independent of rhEPO and to genetically define the neuronal target cells, we expressed a constitutively active form, EPOR ${ }^{\mathrm{R} 129 \mathrm{C}}$ in the postnatal mouse forebrain, using a transgene driven by the $\alpha$-calcium/calmodulin-dependent protein kinase II ( $\alpha$ CaMKII) promoter. The substitution in $\mathrm{EPOR}^{\mathrm{R} 129 \mathrm{C}}$ (CEPOR) confers growth factorindependent survival and tumorigenicity to hematopoietic $\mathrm{Ba} / \mathrm{F} 3$ cells that are normally dependent on interleukin-3 for growth and survival (Longmore et al. , 1994 ;Yoshimura et al. , 1990). The constitutive activity results from the formation of a disulfide-linked receptor homodimer, mimicking the EPO-bound form (Watowich et al. , 1992). Expression of $c E P O R$ thus activates EPOR signaling without requirement of a ligand (Fu et al. , 2009 ;Moucadel and Constantinescu, 2005).

We show here that CEPOR expression in pyramidal neurons of cortex and hippocampus of transgenic (TG) mice prominently enhances higher cognitive performance. Superior cognition is correlated with enhanced paired pulse facilitation and long-term potentiation at the Schaffer collateral CA1 synapse, indicative of increased short- and long-term plasticity. Cognitive augmentation in this genetic model is different from that observed in mice receiving rhEPO injections and comes at the price of higher impulsivity and reduced behavioral control under strong cognitive challenge. We conclude that EPO-EPOR signaling stimulates neuronal plasticity independent of any hematopoietic effects and in addition to its neuroprotective actions.

\section{RESULTS}

\section{Transgenic CEPOR expression in cortical and hippocampal neurons}

To systematically investigate the role of the EPO system in learning, memory and attention, we generated a TG mouse line expressing a constitutively active form of EPOR (CEPOR) under control of the a-CaMKII promoter (Fig.1A), which restricts expression of cEPOR to forebrain pyramidal neurons of postnatal mice. A 
hemagglutinin (HA) tag at the amino terminus of cEPOR allowed monitoring of transgene expression. Of 3 independent lines produced, we analyzed 2 (TG1 and TG2) in detail, which showed a similar spatio-temporal expression pattern of the mutant receptor (Fig.1B and Fig.S1). In fact, cEPOR expression was enriched in cortex and hippocampus (Fig.1B and C) but not in cerebellum and peripheral tissues (Fig.1C). Western blot analysis demonstrated the presence of a $64 \mathrm{kDa}$ mutant receptor in the brains of TG animals that was regulated developmentally over time and augmented in early postnatal days (Fig.1D). Mice lacked any signs of neuropathology, were long-lived, and reproduced well. Animals in both lines showed normal growth and development, body weight, mating behavior and gross brain morphology, virtually identical to that of WT mice (Fig.S2). Moreover, hematocrit levels were comparable between WT and TG mice (WT mice: $45.00 \pm 0.3162 \%, N=5$; TG mice: $46.00 \pm 1.080 \%, p=0.36 ; N=4)$. Thus, behavioral differences would not be secondary to developmental changes of the brain or hyperoxygenation.

\section{Slight hyperactivity but normal basic behavior of cEPOR TG mice}

We first assessed basic behavioral functions, such as locomotor and exploratory activity, anxiety, and motor performance, in cEPOR TG mice in comparison to WT littermates. In the elevated plus maze, there was the expected significant effect of arms (2-way ANOVA, $F(2,90)=66.70 ; p<0.0001)$, but neither a genotype $(p=0.9997)$ nor an interaction effect $(p=0.4748)$, indicating comparable anxiety levels between TG and WT mice (Fig.2A). In the open field test, mice did not differ with respect to the time spent in zones (2-way ANOVA, effect of genotype $p=0.9996$ ) (Fig.2B). However, TG mice showed increased velocity (Mann-Whitney U-test, $p=0.003$ ) (Fig.2B inset) as well as distance travelled (data not shown). There were no differences between groups in exploratory activity, locomotor coordination, motor learning and acoustic startle response (Fig.2C-E).

\section{Enhanced spatial learning and cognitive flexibility}

The novel object recognition test as a relatively crude readout of basic cognition did not reveal any differences between groups (data not shown). However, Morris water maze, a sensitive test for hippocampus-dependent learning/memory processes (Morris, 1984), yielded a significant effect of days (2-way ANOVA for repeated measures $F(7,203)=24.66$; $p<0.0001$ ) as well as a significant effect of genotype (2- 
way ANOVA for repeated measures, $F(1,29)=8.863 ; p=0.006)$ and no interaction effect ( $p=0.87$ ) (Fig.2F). To exclude hyperactivity as a potential confounding variable, we evaluated the distance travelled for locating the hidden platform - a parameter independent of swimming speed. Again, there was a significant effect of genotype (2-way ANOVA for repeated measures, $F(1,29)=6.791 ; p=0.014)$ (Fig.2G) indicating enhanced hippocampus-dependent learning and memory function in cEPOR TG mice. The similar performance level of both groups on day 8 of the hidden platform paradigm $(p=0.8)$ allowed us to further assess cognitive flexibility of cEPOR TG mice using the reversal paradigm. Again, 2-way ANOVA for repeated measures revealed a significant effect of days $(F(7,196)=55.71, p<0.0001)$, significant effect of genotype $(F(1,28)=5.090, p=0.03)$ and a significant interaction effect $(F(7,196)=3.649$, $p=0.001$ ). Post-hoc analysis indicated a significant difference on day 1 (Bonferroni post-hoc test, $p<0.001)$. To further delineate performance of both groups on this critical day 1, we applied a 2-way ANOVA for repeated measures over the 4 trials of this day. Both groups significantly improved over time $(F(3,84)=12.71, p<0.0001)$, however there was a significant genotype effect $(F(1,28)=9.632, p=0.004)$, pointing to faster adaptation of cEPOR TG mice to the new platform position (Fig.2H and inset).

\section{Enhanced synaptic plasticity of the hippocampus}

We hypothesized that the improvements of hippocampal learning are associated with a higher degree of synaptic plasticity. To assess changes in synaptic function and plasticity at the Schaffer collateral-CA1 synapse, extracellular field potential recordings were performed in stratum radiatum of the CA1 subfield of acute hippocampal tissue slices. Basal synaptic function and neuronal excitability were judged on the basis of input-output curves and synaptic plasticity was tested by paired-pulse stimulation and long-term potentiation (LTP)-inducing trains of tetanic stimuli. The amplitude and slope of orthodromically evoked field excitatory postsynaptic potentials (fEPSPs) did not differ significantly among WT and TG mice, and the normalized input-output curves $(10-150 \mu \mathrm{A}$ stimuli) were only slightly rightshifted for cEPOR mice at low stimulation intensities ( $\leq 70 \mu \mathrm{A}$, data not shown). On average, half maximum response amplitudes were obtained with $30 \mu \mathrm{A}$ and $50 \mu \mathrm{A}$ stimuli in WT ( $n=14$ slices) and TG mice ( $n=12$ slices), respectively. 
Clear differences were observed, however, in response to twin-pulse stimulation. In cEPOR TG mice, paired-pulse facilitation with interpulse durations of up to $150 \mathrm{~ms}$ was significantly increased as compared to WT mice (Fig.3A,B). At the shortest interpulse interval tested ( $25 \mathrm{~ms})$, the fEPSP amplitudes increased by $59.3 \pm 15.5 \%$ and $127.9 \pm 22.7 \%$ for WT ( $n=13$ slices) and TG mice $(n=12)$, respectively, and fEPSP slopes increased by $113.6 \pm 20.2 \%$ and $268.2 \pm 45.4 \%$.

LTP-inducing stimuli (3 trains of $100 \mathrm{~Hz}$, each lasting for 1s) also resulted in a more pronounced potentiation of fEPSPs in cEPOR mice, during both the early and the late phase of LTP (Fig.3C,D). Immediately after tetanic stimulation, i.e. the phase being referred to as post-tetanic potentiation (PTP), fEPSP amplitudes were increased by $68.6 \pm 9.4 \%$ and $190.5 \pm 22.4 \%$ for WT and TG mice, respectively, and fEPSP slopes increased by $133.3 \pm 16.0 \%$ and $283.3 \pm 47.9 \%$ (Fig.3D). This initial post-tetanic potentiation decayed over the course of 3-5min and the evoked responses stabilized. One hour after LTP induction, fEPSP amplitudes were still increased by $38.4 \pm 7.4 \%$ and $116.7 \pm 18.7 \%$ and fEPSP slopes were elevated by $51.0 \pm 13.2 \%$ and $140.7 \pm 32.5 \%$ (Fig.3D).

\section{Social memory, attention and higher cognitive capacities}

Would EPO-EPOR signaling also affect higher cortical functions that cannot be easily correlated with electrophysiological readouts? We first addressed social functions in cEPOR TG mice. While there were no differences in social interaction and social approach (data not shown), cEPOR TG mice showed enhanced social memory (Fig.2l). This behavior was not due to increased exploratory activity, since performance in the hole board test did not differ between groups (Fig.2C). Additionally, no differences in olfaction were detected between groups (data not shown) indicating that this finding reflects improved memory function in the area of social interaction.

We next assessed attention and behavioral control in these mice using the 5-choice serial reaction time task (5-CSRTT) (Fig.4A). In this test, mice are trained on an everyday basis over many months to adequately respond to a short $(\leq 1.4 \mathrm{sec})$ light stimulus. Light stimuli appear pseudorandomly in one of five equally distant stimulus holes. Mice are only rewarded if they make a nose poke in the location, where the 
light shows up. To be considered correct, responses have to occur in a short limited time window after stimulus presentation. After reaching stable performance, this trained behavior is challenged via different interfering manipulations (e.g. sound distracters, changing stimulus durations etc).

Training under baseline conditions for 21 days revealed enhanced attentional abilities in cEPOR mice demonstrated by significantly lower reaction times (2-way ANOVA for repeated measures, effect of genotype $F(1,16)=6.649, p=0.005$, Fig.4B). Post-hoc analysis indicated a significant difference in the first trial block (i.e. the first ten trials collapsed over 21 days) $(p<0.001)$. This pattern remained robust even in face of higher cognitive challenges in INT2 (effect of genotype $F(1,12)=8.09$, $p=0.015$; effect of ITI $F(3,36)=15.62$, $p<0.0001$; effect of interaction $F(3,36)=3.757$ ), $p=0.02$, Fig. $4 \mathrm{C}$ ). Post-hoc evaluation revealed significantly lower reaction times in CEPOR TG mice in ITI5 and ITI6 ( $p<0.01$ and $p<0.05$, respectively). Furthermore, cEPOR TG mice tended to show less premature responses in INT2 compared to WT littermates (effect of genotype $F(1,14)=6.658, p=0.02$, Fig.4D). Since progression until 'Recovery4' is performance-dependent, unifying various aspects of learning and cognitive control, we analyzed the proportion of mice finishing this phase until experimental day 250. Survival analysis showed a significant difference between groups $(p=0.03)$, indicating overall faster task progression in cEPOR TG mice (Fig.4E).

\section{Resistance to behavioral distracters}

Having obtained a consistently superior performance of TG mice in the whole series of consecutive 5-CSRTT attentional challenges, we exposed the mice to interfering acoustic stimuli. In the intervention phase 5 (INT5), mice were randomly confronted either with trials, where a sound distracter was simultaneously applied with a short light stimulus, or with trials lacking such distracting auditory stimulus. The effect of the sound distracter on attentional accuracy was investigated. In cEPOR TG mice, the sound distracter did not affect attentional accuracy (Wilcoxon test, $p=0.94$ ) in contrast to WT littermates where sound distraction led to a decrease in accuracy $(p=0.01$, Fig. $4 F)$. Reaction times showed here only borderline significance (effect of genotype $p=0.057$ ). Sound distraction led to a similar increase in reaction times in both groups (effect of sound $F(1,14)=6.572, p=0.023$, Fig.4G). 


\section{Increased impulsivity and reduced behavioral control under challenge}

Experimental cEPOR transgene expression was not ubiquitous in all EPO responsive cells in the brain, but restricted to forebrain pyramidal neurons, suggesting that a natural balance of stimulatory and inhibitory circuits may have been perturbed. We therefore wondered whether overall superior cognitive performance would come at a 'price' and searched for subtle behavioral defects. Indeed, an interesting behavioral abnormality was impulsivity. Under conditions of long inter-trial intervals $(11 \mathrm{sec})$ CEPOR TG mice tended to have more omissions compared to WT littermates (Mann-Whitney U-test, $p=0.02$, Fig.5A). In contrast to their resistance towards attentional distractions, CEPOR TG mice had more premature responses in the task where they were confronted with sound distracters, i.e. responses that occurred before the light-coupled sound appeared (MannWhitney U-test, $p=0.03$, Fig.5B). To address whether signs of increased impulsivity are also detectable in simpler readouts of this behavioral feature, we performed the marble burying test. Indeed, cEPOR TG mice buried more marbles compared to WT littermates (Mann-Whitney U-test, $p=0.047$, Fig.5C). Thus, confining enhanced EPOR activity to glutamatergic cortical projection neurons in the reported experiment may have specific disadvantages, and it will be interesting to compare the here obtained behavioral pattern with the in vivo effects of enhanced EPO-EPOR signaling in other neuronal subpopulations.

\section{DISCUSSION}

In order to investigate how the EPO system influences cognitive performance and synaptic plasticity, and to experimentally prove that previously reported effects of rhEPO on cognition in patients are independent of brain oxygen supply, we created a novel mouse model with constitutive EPOR signaling in cortical neurons that are defined by $\alpha$-CaMKII promoter activity. This way, we were able to specifically mimic EPO system function independent of any ligand in pyramidal neurons of cortex and hippocampus, i.e. regions pivotal for learning and memory processes. In other 
words, we 'over-accentuated' the endogenous action of the EPO system in specific cortical and hippocampal layers, particularly the CA1 subregion (Morishita et al. , 1997), to delineate the contribution of these neuronal subpopulations to the EPO effects on cognition. We found that selective constitutive expression of EPOR in forebrain neurons leads to a phenotype with superior performance in higher cognitive tasks. Behaviorally, this phenotype is accompanied by slightly increased activity and impulsivity. Electrophysiologically, both short- and long-term plasticity at the Schaffer collateral CA1 synapses are significantly increased in cEPOR expressing TG mice.

We performed a detailed and comprehensive behavioral-cognitive analysis of cEPOR TG mice to demonstrate that increased EPO signaling in cortex and hippocampus enhances a whole array of learning and memory processes, as well as cognitive flexibility and attentional capacities, reflected by shorter reaction times and reduced distractibility through competing irrelevant auditory stimuli. Very similar higher cognitive tasks were found improved in human patients upon several months of weekly high-dose intravenous EPO treatment (Ehrenreich et al. , 2007a ;Ehrenreich et al. , 2007b ;Wustenberg et al. , 2010), pointing to specific targets of EPO action on cognition that are common to both mice and humans. Augmented EPOR signaling in cEPOR TG mice also improved social memory, which is partly dependent on hippocampal functions (Kogan et al. , 2000). We note that a recent study reported on better facial recognition performance in patients with major depression following high-dose EPO application (Miskowiak et al. , 2009), supporting social cognition as another selective target of EPO effects across species.

It is important to point out that there are clear differences between EPO effects on higher cognition upon systemic administration to healthy mice (El-Kordi et al. , 2009) as compared to the selective and specific stimulation of the EPO system in forebrain pyramidal neurons reported here. In contrast to mice receiving intraperitoneal EPO injections (El-Kordi et al. , 2009), the cEPOR TG mice did not show improved performance in the (still relatively basic) initial 5-CSRTT training phases. Their superiority, however, was pronounced in the highest cognitive challenge tasks, demanding tremendous attentional capacities. 
Surprisingly, under cognitively most challenging conditions, cEPOR TG mice demonstrated more premature responses as readout of impaired behavioral impulse control (Chudasama et al. , 2003). The slightly hyperactive and impulsive phenotype of cEPOR TG mice was further confirmed by a simple additional assay - the marble burying test. Behavioral consequences of this kind were not noted upon high-dose EPO treatment where the cellular target is defined by the almost ubiquitous presence of EPOR throughout the brain. A potential explanation for the cEPOR TG phenotype of impulsivity and hyperactivity might be the continuous stimulation of the EPO system exclusively in cortical projection neurons. Since the frontal cortex has reciprocal projections to subcortical and basal brain regions (e.g. Kuroda et al. , 1998 ;Rotaru et al. , 2005 ;Uylings et al. , 2003), responsible for locomotion, motivation and impulsivity (for review, see e.g. Berridge and Kringelbach, 2008 ;Chambers et al. , 2003 ;Takakusaki et al. , 2004), the excitation of frontal pyramidal neurons might lead to a relative deficit in the simultaneous inhibitory regulation of these areas, consistent with a disturbance of the homeostatic balance within neuronal networks, resulting in impulsivity and hyperactivity of TG mice. This hypothesis is presently under systematic investigation in our laboratory through selected cEPOR expression in subpopulations of inhibitory interneurons.

EPO and EPOR are expressed at very high levels in the developing central nervous system (Digicaylioglu et al. , 1995 ;Knabe et al. , 2004 ;Knabe et al. , 2005 ;Liu et al. , 1996 ; Liu et al. , 1994). In contrast, their expression is markedly reduced postnatally and remains low in the normal adult brain (Ehrenreich et al. , 2005). Both genes are upregulated under disease conditions in various different cell types in the brain, possibly to exert neuroprotective effects (Bernaudin et al. , 1999 ;Bernaudin et al. , 2002). Our present data indicate that EPO-EPOR signaling serves a role in neuroplasticity, independent of and in addition to its anti-apoptotic neuroprotective tasks. Since the steady-state level of both receptor and ligand is nevertheless low in the uninjured brain, we suggest that this function may also be disease-relevant. We propose a model in which EPO-EPOR induction under disease conditions not only prevents neuronal cell death, but also triggers the enhanced neuronal plasticity that is required to functionally compensate for lost neuronal functions. It is intriguing that this could indicate the strategy of the neocortex, which is known to provide striking functional compensations after injury. This study, together with our previous work, 
also supports a physiological role for EPO in cognitive processes. All this should be exploited to define novel strategies to therapeutically enhance brain plasticity and cognitive performance in disease conditions. 


\section{MATERIALS AND METHODS}

\section{Generation and characterization of transgenic (TG) mice}

$\mathrm{EPOR}^{\mathrm{R} 129 \mathrm{C}}$ (CEPOR) bears a single point mutation at nucleotide 484 , i.e. in the exoplasmic domain, causing a substitution of cysteine for arginine at codon 129 of the $\mathrm{N}$ terminus (R129C). The cDNA sequence of cEPOR, containing a hemagglutinin (HA; YPYDVPDY) tag inserted five residues downstream of the signal peptidase cleavage site (Constantinescu et al. , 2001), was excised with Pacl and Sall from the pMX-HA-cEPOR plasmid. The HA-cEPOR cDNA was inserted into pNN265 plasmid, with a modified multiple cloning site, that carries a 5 ' hybrid intron and a 3 ' intron plus poly-A signal from SV40 through Pacl and Sall sites. Finally, the entire DNA fragment of HA-CEPOR, flanked by a hybrid intron at the 5 ' end and a polyadenylation signal from SV40 at the 3' end was cut out from pNN265 vector using Notl and placed downstream of the $8.5 \mathrm{~kb} \alpha$-CaMKII promoter.

The TG founders were produced by pronuclear injection of the linearized DNA into C57BL6/N ('TG1') or FvB/N ('TG2') zygotes. The analysis of line TG1 mice was performed after ( $\mathrm{N}=4-6)$ backcrosses with $\mathrm{C} 57 \mathrm{BL} 6 / \mathrm{N}$ wildtype mice. The TG1 line was used (because of its clean C57BL6/N background) for the behavioral experiments presented here. The analysis of line TG2 mice was performed after backcrossing $(\mathrm{N}=8-9)$ to $\mathrm{C} 57 \mathrm{BL} 6 / \mathrm{N}$ mice.

The genotype of transgenic offspring was analyzed by PCR of tail genomic DNA using primers specific for the 3 ' end of the $\alpha$-CaMKII promoter sequence (5'-GGGAGGTAGGAAGAGCGATG-3') and the 5' end of the HA-cEPOR cDNA sequence (5'-CACCCTGAGTTTGTCCATCC-3') yielding a 769 bp product. PCR amplification of the tail DNA was carried out with the following conditions: $2 \mathrm{~min}, 94^{\circ} \mathrm{C}$ ( 1 cycle); $30 \mathrm{~s} 94^{\circ} \mathrm{C}, 30 \mathrm{~s} 60^{\circ} \mathrm{C}, 1 \mathrm{~min}, 72^{\circ} \mathrm{C}$ (35 cycles), followed by final extension at $72^{\circ} \mathrm{C}$ for $10 \mathrm{~min}$.

\section{Immunofluorescence}

Wildtype and TG male HA-cEPOR mice were transcardially perfused under deep anesthesia with saline followed by $4 \%$ paraformaldehyde in $0.1 \mathrm{M}$ sodium phosphate buffer (PBS) ( $\mathrm{pH} \mathrm{7.4).} \mathrm{Brains} \mathrm{were} \mathrm{removed} \mathrm{from} \mathrm{the} \mathrm{skulls,} \mathrm{postfixed} \mathrm{in} \mathrm{4 \%} \mathrm{PFA}$ overnight at $4{ }^{\circ} \mathrm{C}$ and subsequently cryoprotected in $30 \%$ sucrose/PBS solution. After 
being frozen on dry ice, coronal cryosections $(30 \mu \mathrm{m})$ were collected, washed briefly with PBS and incubated for $40 \mathrm{~min}$ at room temperature (RT) with $0.1 \%$ glycine (Merck, Darmstadt, Germany), $0.1 \%$ Triton X-100 in PBS. Sections were then immersed for $1 \mathrm{~h}$ at RT in blocking solution [5\% normal horse serum (NHS), $0.1 \%$ Triton X-100 in PBS] and incubated with mouse monoclonal anti-HA (1:500, Covance, Hiss Diagnostics, Freiburg, Germany) diluted in 3\% NHS, $0.1 \%$ Triton X$100 /$ PBS overnight at $4^{\circ} \mathrm{C}$. After washing with PBS, sections were treated with antimouse Cy3-coupled secondary antibody (1:1000, Jackson ImmunoResearch Laboratories-Dianova, Hamburg, Germany) for $1 \mathrm{~h}$ at RT. Following PBS washes, sections were mounted on Super Frost microscopic slides, air dried and coverslipped using Aqua-Poly/Mount (Polysciences, Eppelheim, Germany). Sections were imaged with a fluorescence stereomicroscope (Leica MZ16 FA, Wetzlar, Germany).

\section{Protein extraction and immunoblotting}

Wildtype and TG HA-cEPOR male mice were sacrificed by cervical dislocation. The tissue samples were dissected and immediately frozen on dry ice. For immunoblotting, tissue samples including hippocampus, cortex, cerebellum, liver and kidney, were homogenized in lysis buffer $(50 \mathrm{mM}$ Tris $\mathrm{HCL}(\mathrm{pH} 8.3), 150 \mathrm{mM} \mathrm{NaCl}$, $40 \mathrm{mM} \mathrm{NaF}, 5 \mathrm{mM}$ EDTA, $5 \mathrm{mM}$ EGTA, $1 \mathrm{mM} \mathrm{Na} \mathrm{NO}_{4}, \quad 1 \%$ Igepal, $0.1 \%$ Natriumdesoxycholat, $0.1 \% \mathrm{SDS}$ ] containing $1 \mathrm{mM}$ phenylmethysulfonylfluoride, $10 \mu \mathrm{g} / \mathrm{ml}$ aprotinin and $0.1 \mathrm{mg} / \mathrm{ml}$ leupeptin) using an Ultra-turrax homogenizer (Kinematica, Luzern, Switzerland). The lysates were centrifuged (1200rpm) for $45 \mathrm{~min}$ at $4^{\circ} \mathrm{C}$. The supernatant was collected and mixed with 3 volumes of Laemmli buffer [250 mM Tris HCL (pH 8.3), 8\% SDS, 40\% glycerol, 20\% 2-mercaptoethanol, $0.04 \%$ pyronin $\mathrm{Y}$ ], and boiled for $10 \mathrm{~min}$ at $70^{\circ} \mathrm{C}$ for immunoblotting. The protein samples were run on NuPAGE 4-12\% Bis-Tris Gel (Invitrogen, Karlsruhe, Germany) for $1 \mathrm{~h}$ at $200 \mathrm{~V}$ and transferred to a nitrocellulose membrane. After blocking with $5 \%$ milk in Tween 20-Tris-buffered saline (TTBS) for $1 \mathrm{~h}$ at RT, membranes were incubated in primary antibodies for mouse rat monoclonal anti-HA (1:500, Roche, Mannheim, Germany) with mouse monoclonal anti-GAPDH (1:10000, Assay Designs/Stressgen, Ann Arbor, MI, USA) as an internal control. Immunoreactive bands were visualized using secondary antibodies coupled to horseradish peroxidase by enhanced chemoluminescence (Amersham, Freiburg, Germany). 
RNA isolation and expression analysis by reverse transcriptase PCR (RT-PCR) Brains of E12, E17, P0, P14 and adult (2 months old) TG male HA-cEPOR mice were rapidly frozen after being sacrificed. Tissue was homogenized in Trizol using an Ultra-turrax homogenizer (Kinematica, Luzern, Switzerland). Total RNA was isolated by using the RNeasyPlus kit (Qiagen, Hilden, Germany). During RNA isolation, on-column DNase digestion was performed with the RNase-free DNase set (Qiagen, Hilden, Germany). cDNA was prepared using N9 random and Oligo(dT)18 primers. HA-cEPOR expression was detected by PCR using the sense (5' CTACCCATACGACGTCCCAG 3') and antisense (5' GCGTCCAGGAGCACTACTTC 3') primers specific for the transgene, yielding a 362bp product. GAPDH cDNA was amplified as internal control using the sense 5' TGCCAAGGCTGTGGGCAAGG 3' and antisense 5' TGTTGGGGGCCGAGTTGGGA 3' primers (431bp). PCR amplification was done with following conditions: $2 \mathrm{~min}, 94^{\circ} \mathrm{C}$ ( 1 cycle); $45 \mathrm{~s} 94^{\circ} \mathrm{C}, 45 \mathrm{~s}$ $58^{\circ} \mathrm{C}, 1 \mathrm{~min} 72^{\circ} \mathrm{C}$ (30 cycles), followed by final extension at $72^{\circ} \mathrm{C}$ for $10 \mathrm{~min}$.

\section{Slice preparation and electrophysiological recordings}

Synaptic function and plasticity were assessed in acute brain tissue slices. Adult male wildtype and TG HA-cEPOR mice (3-4 months old) were decapitated under deep ether anesthesia, the brain was rapidly removed from the skull and placed in chilled artificial cerebrospinal fluid (ACSF) for 1-2min. Acute neocortical/hippocampal tissue slices $(400 \mu \mathrm{m}$ thick transverse slices) were cut from the forebrain using a vibroslicer (752M Vibroslice, Campden Instruments, Loughborough, UK). The slices were then separated in the sagittal midline, transferred to an Oslo style interface recording chamber and left undisturbed for at least $90 \mathrm{~min}$ to ensure recovery from surgical trauma. Recording chamber was kept at a temperature of $32-33^{\circ} \mathrm{C}$, continuously aerated with $95 \% \mathrm{O}_{2}-5 \% \quad \mathrm{CO}_{2}(400 \mathrm{ml} / \mathrm{min})$, and perfused with oxygenated ACSF (3-4ml/min). The ACSF contained (in $\mathrm{mM}$ ): $130 \mathrm{NaCl}, 3.5 \mathrm{KCl}$, $1.25 \mathrm{NaH}_{2} \mathrm{PO}_{4}, 24 \mathrm{NaHCO}_{3}, 1.2 \mathrm{CaCl}_{2}, 1.2 \mathrm{MgSO}_{4}$, and 10 dextrose; aerated with $95 \% \mathrm{O}_{2}-5 \% \mathrm{CO}_{2}$ to adjust $\mathrm{pH}$ to 7.4 .

Orthodromically evoked field excitatory postsynaptic potentials (fEPSPs) were elicited by stimulation of Schaffer collaterals and recorded in stratum radiatum of the CA1 subfield with a locally constructed extracellular DC potential amplifier as described earlier (Adamcio et al. , 2008 ;Hepp et al. , 2005). Unipolar stimuli of 
$0.1 \mathrm{~ms}$ duration, negative polarity and $10-150 \mu \mathrm{A}$ amplitude were generated by a stimulator (Grass S88 stimulator equipped with PSIU6 stimulus isolation units, Grass Instruments, Astro-Med Inc., Rodgau, Germany) and delivered via stimulation electrodes made from steel microwire $(50 \mu \mathrm{m}$ diameter, AM-Systems, Carlsborg WA, USA; (Muller and Somjen, 1998)). Extracellular recording electrodes were pulled from thin-walled borosilicate glass capillaries (GC150TF-10, Harvard Apparatus, Holliston MA, USA) using a horizontal electrode puller (P-97 Flaming/Brown Micropipette Puller, Sutter Instruments, Novato CA, USA). They were filled with ACSF and their tips were trimmed to a final resistance of $\sim 5 \mathrm{M} \Omega$. Evoked responses were sampled at an acquisition rate of $20 \mathrm{kHz}$ using an Axon Instruments Digitizer 1322A and PClamp 9.2 software (Molecular Devices Corporation, Sunnyvale CA, USA). Synaptic function and neuronal excitability were assessed by recording input-

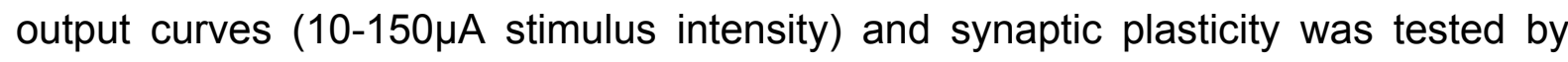
inducing paired-pulse facilitation (PPF) and long-term potentiation (LTP). For PPF and LTP-recordings, stimulation intensity was adjusted to obtain half-maximum response amplitudes. In PPF recordings, the inter-stimulus interval was varied in the range of $25-200 \mathrm{~ms}$. LTP was induced by three $1 \mathrm{~s}$ lasting $100 \mathrm{~Hz}$ trains, separated by 20 s each, and fEPSPs were then recorded for $60 \mathrm{~min}$. To improve the signal to noise ratio of the recordings, 4 consecutive sweeps were averaged online (Muller and Somjen, 1998); individual stimuli were delivered every 5s (input-output curves and PPF) or 15s (LTP). To quantify changes in synaptic function and plasticity, the amplitude of the EPSPs and their slope (within the $20-80 \%$ range of the falling phase) were analyzed using PClamp 9.2 software (Molecular Devices).

\section{Behavioral testing}

All experiments were approved by the local Animal Care and Use Committee in accordance with the German Animal Protection Law. For behavioral testing, mice were housed in groups of 3-5 in standard plastic cages, food and water ad libitum (except for the 5-choice training period, see below). The temperature in the colony room was maintained at $20-22^{\circ} \mathrm{C}$, with a $12 \mathrm{~h}$ light-dark cycle (light on at 7:00am). Behavioral experiments were conducted by an investigator, blinded to the genotype, during the light phase of the day (between 8:00am and 17:00pm). The order of testing was as follows: Elevated plus maze, open field, hole board, rotarod, pre-pulse 
inhibition, social interaction, novel object recognition test, Morris water maze, the 5choice serial reaction time task and marble burying test. Age of mice at the beginning of testing was 11-12 weeks. Inter-test interval was at least 1-2 days.

Elevated plus maze: In this test of anxiety, mice were placed in the central platform, facing an open arm of the plus-maze (made of grey plastic with a $5 \times 5 \mathrm{~cm}$ central platform, $30 \times 5 \mathrm{~cm}$ open arms and $30 \times 5 \times 15 \mathrm{~cm}$ closed arms; illumination 120lx). The behavior was recorded for $5 \mathrm{~min}$ by an overhead video camera and a PC equipped with "Viewer 2" software (Biobserve $\mathrm{GmbH}$, Bonn, Germany) to calculate the time spent in open or closed arms, distance traveled, number of arm visits, and velocity. The proportion of time spent in open arms was used to estimate open arm aversion (fear equivalent).

Open field: Spontaneous activity in the open field was tested in a grey Perspex arena $(120 \mathrm{~cm}$ in diameter, $25 \mathrm{~cm}$ high; illumination $120 \mathrm{~lx})$. Mice were placed in the center and allowed to explore the open field for $7 \mathrm{~min}$. The behavior was recorded by a PC-linked overhead video camera. "Viewer 2" software was used to calculate velocity, distance traveled, and time spent in central, intermediate or peripheral zones of the open field.

Hole board: The hole board test measures exploratory activity. The apparatus consisted of a $51 \times 51 \times 33 \mathrm{~cm}$ transparent Perspex chamber with a non-transparent floor, with 16 equally spaced holes, $2 \mathrm{~cm}$ in diameter, $2 \mathrm{~cm}$ deep. Mice were allowed to explore the chamber for $5 \mathrm{~min}$ and the number of explored holes (head dips) was registered by a computer software (TSE GmbH, Bad Homburg, Germany). The illumination in the testing room was $1201 x$.

Rotarod: The rotarod test examines motor function, balance, and coordination. It comprised a rotating drum (Ugo Basile, Comerio, Varese, Italy), which was accelerated from 4 to $40 \mathrm{rpm}$ over $5 \mathrm{~min}$. Mice were placed individually on the drum and the latency of falling off the drum was recorded using a stop-watch. To assess motor learning, the rotarod test was repeated $24 \mathrm{~h}$ later.

Pre-pulse inhibition test: In this test of sensorimotor gating, individual mice were placed in small metal cages $(90 \times 40 \times 40 \mathrm{~mm})$ to restrict major movements and exploratory behavior. The cages were equipped with a movable platform floor attached to a sensor that records vertical movements of the floor. The cages were placed in 4 sound-attenuating isolation cabinets (TSE GmbH, Bad Homburg, Germany). Startle reflexes were evoked by acoustic stimuli delivered from a 
loudspeaker that was suspended above the cage and connected to an acoustic generator. The startle reaction to an acoustic stimulus, which evokes a movement of the platform and a transient force resulting from this movement of the platform, was recorded with a computer during a recording window of $260 \mathrm{~ms}$ (beginning with the onset of pre-pulse) and stored for further evaluation. The recording window was defined from the onset of the acoustic stimulus. An experimental session consisted of a $2 \mathrm{~min}$ habituation to $65 \mathrm{~dB}$ background white noise (continuous throughout the session), followed by a baseline recording for $1 \mathrm{~min}$ at background noise. After baseline recording, 6 pulse-alone trials using startle stimuli of $120 \mathrm{~dB}$ intensity and $40 \mathrm{~ms}$ duration were applied in order to decrease influence of within-session habituation. These data were not included in the analysis of the pre-pulse inhibition. For tests of pre-pulse inhibition, the $120 \mathrm{~dB} / 40 \mathrm{~ms}$ startle pulse was applied either alone or preceded by a pre-pulse stimulus of $70 \mathrm{db}, 75 \mathrm{db}$, or $80 \mathrm{~dB}$ sound pressure level and $20 \mathrm{~ms}$ duration. An interval of $100 \mathrm{~ms}$ with background white noise was employed between each pre-pulse and pulse stimulus. The trials were presented in a pseudorandom order with an interval ranging from 8 to 22s. The amplitude of the startle response (expressed in arbitrary units) was defined as the difference between the maximum force detected during a recording window and the force measured immediately before the stimulus onset. Amplitudes were averaged for each individual animal, separately for both types of trials (i.e. stimulus alone or stimulus preceded by a pre-pulse). Pre-pulse inhibition was calculated as the percentage of the startle response using the following formula: \% pre-pulse inhibition $=100-[$ (startle amplitude after pre-pulse and pulse) / (startle amplitude after pulse only) x 100].

Social interaction: Sociability and social memory were tested as described in detail elsewhere ((e.g. Moy et al. , 2004)). The social testing arena was a rectangular, 3chambered box. Each chamber was $20 \times 40 \times 22 \mathrm{~cm}$ in size. Dividing walls were made from clear Plexiglas, with rectangular openings $(35 \times 35 \mathrm{~mm})$ allowing access into each chamber. The chambers of the arena were cleaned, and fresh paper chip bedding was added between trials. The test mouse was first placed in the middle chamber and allowed to explore for $5 \mathrm{~min}$. The openings into the two-side chambers were obstructed by plastic boxes during this habituation phase. After the habituation period, an unfamiliar $\mathrm{C} 57 \mathrm{BL} / 6 \mathrm{NCrl}$ male mouse (stranger 1) without prior contact with the subject mouse was placed in one of the side chambers. The location of stranger 1 in the left versus right side chamber was systematically alternated 
between trials. The stranger mouse was enclosed in a small $(60 \times 60 \times 100 \mathrm{~mm})$, rectangular wire cage, which allowed nose contact through the bars but prevented fighting. The animals serving as strangers had previously been habituated to placement in the small cage. An identical empty wire cage was placed in the opposite chamber. A heavy cup was placed on the top of each of the small wire cages to prevent climbing by the test mice. Both openings to the side chambers were then unblocked, and the subject mouse was allowed to explore the entire social test arena for a 10min-session. The amount of time spent in each chamber and the number of entries into each chamber were recorded by the video tracking system "Viewer 2" (Biobserve GmbH). An entry was defined as all 4 paws in one chamber. At the end of the first $10 \mathrm{~min}$-trial, each mouse was tested in a second $10 \mathrm{~min}$-session to quantify social preference for a new stranger. A second, unfamiliar mouse (stranger 2) was placed into the previously empty wire cage. The test mouse had a choice between the first, already explored mouse (familiar stranger 1), and the novel unfamiliar mouse (new stranger 2). As described above, measures were taken of the amount of time spent in each chamber and the number of transitions between chambers of the apparatus during the second $10 \mathrm{~min}$-session. Based on the amount of time spent in each chamber, a 'sociability index' and a 'social memory index' (with a value of 0 meaning no preference) were calculated according to the following formulas:

Sociability index $=\left(\left(\right.\right.$ Time $_{\text {stranger }} /\left(\right.$ Time $_{\text {stranger }}+$ Time $\left.\left.\left._{\text {empty }}\right)\right) \times 100\right)-50$. Memory index $=\left(\left(\right.\right.$ Time $_{\text {novel mouse }} /\left(\right.$ Time $_{\text {novel mouse }}+$ Time $\left.\left.\left._{\text {familiar mouse }}\right)\right) \times 100\right)-50$.

Novel object recognition test: As a test for recognition memory, the novel object recognition test consisted of a training phase and a testing phase. First, mice were habituated to the experimental apparatus for $10 \mathrm{~min}$ in the absence of any objects. The experimental apparatus was a rectangular open field $(40 \times 40 \times 40 \mathrm{~cm})$ made of grey plastic. During the training phase, mice were placed in the experimental apparatus with 1 object and allowed to explore for $10 \mathrm{~min}$. The object was thoroughly cleaned between trials to make sure no olfactory cues were present. Retention was tested immediately after training and 30min later. During these retention tests, the mouse explored the experimental apparatus for 10min in the presence of 1 familiar and 1 novel object. An independent set of objects was used for training and testing of each retention interval. The location of the object was counterbalanced so that one-half of the animals in each group saw the novel object on the left side of the 
apparatus, and the other half saw the novel object on the right side of the apparatus. A mouse was scored as exploring an object when its head was oriented toward the object within a distance of $1 \mathrm{~cm}$ or closer. Preference index, a ratio of the amount of time spent exploring the new object over the total time spent exploring both objects during the retention test was used as a measure of recognition memory.

Morris water maze: Spatial learning and memory was assessed in a water maze (Morris, 1984). A large circular tank (diameter $1.2 \mathrm{~m}$, depth $0.4 \mathrm{~m}$ ) was filled with opaque water $\left(25 \pm 1^{\circ} \mathrm{C}\right.$, depth $\left.0.3 \mathrm{~m}\right)$ and the escape platform $(10 \times 10 \mathrm{~cm})$ was submerged $1 \mathrm{~cm}$ below the surface. The swim patterns were monitored by a computer and the video-tracking system "Viewer 2". The escape latency, swim speed, path length, and trajectory of swimming were recorded for each mouse. During the first 2 days, mice were trained to swim to a clearly visible platform (visible platform task) that was marked with a $15 \mathrm{~cm}$ high black flag and placed pseudorandomly in different locations across trials (non-spatial training). The extra-maze cues were hidden during these trials. After 2 days of visible platform training, hidden platform training (spatial training) was performed. For 8 days, mice were trained to find a hidden platform (i.e. the flag was removed) that was located in the center of one of the 4 quadrants of the pool. The location of the platform was fixed throughout testing. Mice had to navigate using extra-maze cues that were placed on the walls of the testing room. Every day, mice went through 4 trials with an inter-trial interval of $5 \mathrm{~min}$. The mice were placed into the pool facing the side wall randomly at 1 of 4 start locations and allowed to swim until they found the platform, or for a maximum of $90 \mathrm{~s}$. Any mouse that failed to find the platform within 90 s was guided to the platform. The animal then remained on the platform for 20 s before being removed from the pool. The next day after completion of the hidden platform training, a probe trial was conducted in order to determine whether mice used a spatial strategy to find the platform or not. The platform was removed from the pool and the mice were allowed to swim freely for $90 \mathrm{~s}$. The percentage of time spent in each quadrant of the pool as well as the number of times the mice crossed the former position of the hidden platform were recorded. In order to investigate the flexibility of cognitive processes in mice, the reversal water maze test was performed. The experimental procedure was identical to the one used for the hidden platform training with the exception that the escape platform was moved from the original position to the neighbouring quadrant. 
The 5-choice serial reaction time task (5-CSRTT) measures higher brain functions, ranging from various discrete learning/memory to attentional paradigms (Humby et al. , 2005 ;Robbins, 2002). Mice were trained in an operant chamber (width $15.5 \mathrm{~cm}$, depth $20 \mathrm{~cm}$, height $18 \mathrm{~cm}$; Med Associates Inc, St. Albans, USA), enclosed in a sound attenuating box and connected to a Fujitsu Siemens PC. One wall of the operant chamber had a curved shape and carried an array of five stimulus holes. The stimulus holes were $1.2 \mathrm{~cm}$ in diameter and contained a LED stimulus light (depth $1 \mathrm{~cm}$ ) in the rear. Infrared photocell pairs were located at $4 \mathrm{~mm}$ from the entrance of the stimulus holes and detected nose pokes of mice into the holes. The wall opposite to the stimulus holes contained a magazine cup, also with a photocell detector of head entries, in which liquid reward (4\% sucrose solution) was delivered always simultaneously with illumination of the magazine. The house light was located 32cm above the magazine.

Habituation and magazine training: Two days before starting training, mice were habituated to the liquid reward of $4 \%$ sucrose solution in their home cages over night. The day before starting magazine training, sucrose bottles were removed and mice were water deprived. Water deprivation was applied during the entire experimental period. Immediately after finishing the daily test sessions, mice were given water in individual cages for 20min. Magazine training consisted of four consecutive phases (M1-M4), 1 phase per day, each lasting for 15min, with all stimulus holes closed. In the first phase (M1), liquid reward was delivered $(10 \mu \mathrm{l})$ upon initiation of the training session. In the second phase of magazine training (M2), the number of potential rewards was increased, with a fixed interval of $118 \mathrm{sec}$ between reward presentations. A head entry into the magazine was required to collect the reward. In the third phase (M3), the fixed interval was replaced by a head entry-dependent interval of $100 \mathrm{sec}$ to obtain reward. In the last phase (M4), this interval was further reduced to $50 \mathrm{sec}$, ideally yielding a consistently increasing number of head entries. Head entries into the magazine together with reward consumption were taken as indicators for associating the magazine with reward delivery.

Shaping phases (operant and discriminant learning): During shaping, mice were trained to perform a nose poke into an illuminated stimulus hole in order to obtain reward. The shaping procedure consisted of two phases (each extending over several days, dependent on individual performance, and with a daily session 
duration of $30 \mathrm{~min}$ ) where mice were taught to associate nose poking into an illuminated hole with reward (phase S1), and then trained to discriminate between those nose pokes that lead to reward (illuminated holes) and those that do not (unlit holes) (phase S2). Throughout shaping all stimulus holes were open. During S1, all stimulus lights were on. Any nose poke in a stimulus hole was rewarded. The intertrial interval (time from pick-up of reward to next stimulus hole illumination) was set to 8sec. During S2, presentation of lit and unlit holes was conducted in a pseudorandom manner. Mice were only rewarded upon nose poking into a lit stimulus hole. Performing a nose poke in an unlit hole led to switch-off of the house light for $5 \mathrm{sec}$. Mice in $\mathrm{S} 1$ were moved to the next training phase once they had reached 35-40 nose pokes each on 3 consecutive days. The number of trials in S2 was 60 per day and training in this phase was terminated when mice had arrived at a stable performance of $\geq 70 \%$ correct responses for three consecutive days.

5-CSRTT training: The training session started with illumination of magazine light and presentation of $4 \%$ sucrose solution. Head entry started the trial. At $8 \mathrm{sec}$ after head entry, light (initially set to 16sec) was randomly presented in one of the 5 stimulus holes. A correct response, i.e. nose poking into the lit hole, led to reward $(6 \mu l)$ and the next trial start after 8sec (inter-trial interval, ITI). Nose poking in an unlit stimulus hole, i.e. an incorrect response, led to extinguishing the house light for $5 \mathrm{sec}$ (time-out) and no reward. Further nose pokes during time-out extended that period for additional $5 \mathrm{sec}$ each. If a mouse did not respond by nose poking into any of the holes during stimulus presentation, an omission was counted. As a consequence, no reward was presented. Also omissions provoked time-out. A training session was terminated after $30 \mathrm{~min}$ or upon performing 60 trials, whatever came first. Mice were trained in the phase with $16 \mathrm{sec}$ stimulus duration until they reached clearly defined performance criteria $(\geq 75 \%$ accuracy [correct responses/correct + incorrect responses * 100], $\leq 20 \%$ omissions and at least 50 trials performed over 3 consecutive days). Six such phases followed with gradually declining stimulus duration up to $1.4 \mathrm{sec}(16,8,4,2,1.8$ and $1.4 \mathrm{sec})$. In the first phases, mice had time to respond as long as the stimulus light was on. For phases with stimulus duration below $5 \mathrm{sec}$, the response time (so called limited hold) was added up to $5 \mathrm{sec}$.

Baseline training and attentional manipulations

After the acquisition phase, which was terminated by reaching stable performance criteria in the $1.4 \mathrm{sec}$-phase (see above), mice were trained at 'baseline parameters', 
i.e. stimulus duration of $1.4 \mathrm{sec}$, an ITI of $8 \mathrm{sec}$ and a maximum of 60 trials. This training was performance-independent, lasted for 21 days ( 1 session/day) and was conducted to further stabilize performance of mice (Wrenn et al. , 2006). Immediately after baseline training, attentional intervention phases (INT1-5) succeeded in the following manner: (INT1) variable, long ITI (8, 9, 10 and 11sec); (INT2) variable, short ITI (5, 6, 7 and 8sec); (INT3) variable, short stimulus duration (1.4, 1.0, 0.6 and 0.2sec); (INT4) variable long ITI (8, 9, 10 and 11sec) coupled with variable short stimulus duration $(1.4,1.0,0.6$ and $0.2 \mathrm{sec})$ and (INT5) short stimulus duration (0.6sec) applied simultaneously with a sound distracter $(80 \mathrm{~dB}$, white noise). INT1, INT2, INT3 and INT5 lasted for 4 days (1 session/day), while INT4 was conducted for 15 days ( 1 session/day). Between 2 intervention phases, mice were kept in a 'recovery' phase, consisting of baseline parameters, until they re-reached the above described baseline performance criteria. This was done to rule out the impact of motivational and learning factors resulting from the prior intervention phase (Humby et al. , 1999).

Marble burying test: The marble burying test is used to assess stereotypies and obsessive-compulsive behaviors in mice (Deacon, 2006). Mice were tested in plastic cages $(34.5 \times 56.5 \times 18 \mathrm{~cm})$ filled with $5 \mathrm{~cm}$ deep wood chip bedding. 24 glass marbles evenly spaced (4cm apart) were placed on the surface. Individual mice were put in the cage and left there for 30min. Illumination was dimmed (6lx). The number of buried marbles (to 2/3 their depth) during this time was counted.

\section{Statistical analysis}

Statistical analysis was performed using the statistical programs SPSS for windows, release 16 (SPSS Inc., Chicago, USA) and GraphPad Prism version 4.00 for Windows, (GraphPad Software, San Diego, USA). We applied 2-way ANOVA for repeated measures, Kruskal-Wallis test, chi²-test, Mann-Whitney U-Test and survival analysis where indicated. Bonferroni and Dunn's multiple comparison tests were used for post-hoc analysis. Threshold for significance was $p<0.05$. All data are presented as mean \pm SEM (standard error of the mean). 


\section{ACKNOWLEDGEMENTS}

This study was supported by the Max Planck Society and the DFG-Research Center for Molecular Physiology of the Brain (CMBP). The authors thank Swetlana Sperling and Anja Ronnenberg for their excellent technical help.

\section{COMPETING INTEREST}

User patents on EPO in schizophrenia and MS have been submitted. 


\section{Figure Legends}

Figure 1: Construction and characterization of HA-cEPOR TG mice ('TG1').

(A) Construct HA-cEPOR used for production of HA-cEPOR TG mice. HA-cEPOR, flanked by a hybrid intron at the 5' end and a polyadenylation signal at the 3 ' end was placed under the control of the $\alpha$-CaMKII promoter. (B) Forebrain-specific expression of HA-cEPOR transgene revealed by immunohistochemistry. A monoclonal antibody against the HA-tag was used to stain coronal sections of the hippocampus. Expectedly, HA-cEPOR expression is absent in WT mice [(a) and (b) are magnifications of the respective regions of interest]. In TG mice, HA-cEPOR expression is restricted to pyramidal neurons of the cortex (c), CA1 (d) as well as CA3 subregions of the hippocampus and granular layer of the dentate gyrus. Scale bars; $100 \mu \mathrm{m}$ and $500 \mu \mathrm{m}$. (C) Tissue-specific expression of HA-cEPOR mRNA (top) and protein (bottom) in TG mice. TG mRNA expression was detected by PCR using TG specific primers, yielding a 362bp product. Western blot analysis of HA-cEPOR using a monoclonal antibody against HA-tag revealed a $64 \mathrm{kDa}$ band. HA-cEPOR mRNA and protein were expressed in cortex (CX) and hippocampus (HP) of TG mice but not in cerebellum (CB) or in peripheral tissues (LIV: liver; KID: kidney). GAPDH was used as the internal control for both mRNA (431bp) and protein (36kDa) expression analysis. (D) Developmental regulation of the HA-cEPOR transgene. HA-cEPOR transgenic mRNA and protein expression was not seen in fetal tissue ('embryonic' day 12 and 17), but detected at early postnatal days (P0, P14) and remained constant until adulthood.

Figure 2: Essentially normal basic behavior of cEPOR TG mice contrasts the distinctly superior cognitive performance.

TG mice were normal in basic behavioral functions (A-E) except for slight hyperactivity, as demonstrated by increased velocity in the open field (B). In basic cognitive tasks, i.e. spatial learning/memory and cognitive flexibility as evaluated by Morris water maze (F-H) and social memory (I), CEPOR TG mice were significantly superior to WT. $\mathrm{N}=13-18$ per group; mean \pm SEM presented. 
Figure 3: cEPOR mice reveal improved synaptic short- and long-term plasticity $(A, B)$ Short-term plasticity as tested by paired-pulse facilitation was markedly enhanced in CEPOR mice. The amplitude (A) and slope (B) of the second EPSP are normalized to the first EPSP of a given twin pulse. Plotted are the averages of 13 (wildtype) and 12 slices (CEPOR TG). Error bars represent SEM and the level of significance of the changes observed is indicated. The sample responses shown in the inset were elicited by $30 \mu \mathrm{A}$ stimuli separated by $25 \mathrm{~ms}$; stimulation artefacts are truncated. (C,D) Also, post tetanic potentiation (PTP) as well as long-term potentiation (LTP) was more pronounced in CEPOR mice. LTP was induced at time 0 ; plotted are the normalized averages of 11 slices for each genotype. The sample responses in the inset show EPSPs under baseline conditions, immediately after the LTP-inducing stimulus, i.e. the phase of PTP, and 60min after stimulation. Responses were elicited by $60 \mu \mathrm{A}$ stimuli, scaling is identical to panel $\mathrm{B}$.

Figure 4: cEPOR TG mice perform better in the attentional interventions of the 5-choice serial reaction time task (5-CSRTT).

(A) Experimental design depicting the standard baseline training steps in 5-CSRTT as well as attentional manipulations. (B,C) CEPOR TG mice showed better attentional performance reflected by shorter reaction times. (D) They displayed less premature responses under lower cognitive demands and (E) were overall faster in progressing through the consecutive training steps. $(\mathbf{F}, \mathbf{G})$ Additionally, they were less distractible and more resistant towards irrelevant competing auditory stimuli. $\mathrm{N}=7-11$ per group; mean \pm SEM presented.

Figure 5: cEPOR TG mice show increased impulsivity and reduced behavioral control under conditions of high cognitive challenge.

(A) CEPOR TG mice were more distractible in trials comprising low frequency stimulus presentations (longer inter-trial interval) and (B) more impulsive in sessions including an irrelevant auditory distracter. (C) Additional evaluation of these phenomena using the marble burying test confirmed the impulsive phenotype. $\mathrm{N}=7$ 11 per group; mean \pm SEM presented. 
Figure S1: Expression of HA-cEPOR in the 'TG2' line. Forebrain-specific expression of HA-cEPOR transgene also in line 'TG2' was revealed by immunohistochemistry. A monoclonal antibody against the HA-tag was used to stain coronal sections of hippocampus. HA-cEPOR expression is absent in WT mice (A and B). Similar to the line 'TG1', HA-CEPOR expression is restricted to the pyramidal neurons of cortex (C), CA1 (D), CA3 subregions of hippocampus and granular layer of dentate gyrus. Scale bars; $100 \mu \mathrm{m}$ and $500 \mu \mathrm{m}$.

Figure S2: Overexpression of cEPOR does not affect overall brain morphology. (A) Coronal sections from WT and TG mice were stained with haematoxylin-eosin and (B) Luxol Fast Blue. General brain morphology and myelin architecture were comparable between WT and TG mice. Scale bars; $1 \mathrm{~mm}$. 


\section{REFERENCES}

Adamcio B, Sargin D, Stradomska A, Medrihan L, Gertler C, Theis F, et al. Erythropoietin enhances hippocampal long-term potentiation and memory. BMC Biol. 2008;6:37.

Banks WA, Jumbe NL, Farrell CL, Niehoff ML, Heatherington AC. Passage of erythropoietic agents across the blood-brain barrier: a comparison of human and murine erythropoietin and the analog darbepoetin alfa. Eur J Pharmacol. 2004 Nov 28;505(1-3):93101.

Bernaudin M, Marti HH, Roussel S, Divoux D, Nouvelot A, MacKenzie ET, et al. A potential role for erythropoietin in focal permanent cerebral ischemia in mice. J Cereb Blood Flow Metab. 1999 Jun;19(6):643-51.

Bernaudin M, Nedelec AS, Divoux D, MacKenzie ET, Petit E, Schumann-Bard P. Normobaric hypoxia induces tolerance to focal permanent cerebral ischemia in association with an increased expression of hypoxia-inducible factor- 1 and its target genes, erythropoietin and VEGF, in the adult mouse brain. J Cereb Blood Flow Metab. 2002 Apr;22(4):393-403.

Berridge KC, Kringelbach ML. Affective neuroscience of pleasure: reward in humans and animals. Psychopharmacology (Berl). 2008 Aug;199(3):457-80.

Brines M, Cerami A. Emerging biological roles for erythropoietin in the nervous system. Nat Rev Neurosci. 2005 Jun;6(6):484-94.

Brines ML, Ghezzi P, Keenan S, Agnello D, de Lanerolle NC, Cerami C, et al. Erythropoietin crosses the blood-brain barrier to protect against experimental brain injury. Proc Natl Acad Sci U S A. 2000 Sep 12;97(19):10526-31.

Byts N, Samoylenko A, Fasshauer T, Ivanisevic M, Hennighausen L, Ehrenreich H, et al. Essential role for Stat5 in the neurotrophic but not in the neuroprotective effect of erythropoietin. Cell Death Differ. 2008 Apr;15(4):783-92.

Chambers RA, Taylor JR, Potenza MN. Developmental neurocircuitry of motivation in adolescence: a critical period of addiction vulnerability. Am J Psychiatry. 2003 Jun;160(6):1041-52.

Chudasama Y, Passetti F, Rhodes SE, Lopian D, Desai A, Robbins TW. Dissociable aspects of performance on the 5-choice serial reaction time task following lesions of the dorsal anterior cingulate, infralimbic and orbitofrontal cortex in the rat: differential effects on selectivity, impulsivity and compulsivity. Behav Brain Res. 2003 Nov 30;146(1-2):105-19.

Constantinescu SN, Keren T, Socolovsky M, Nam H, Henis YI, Lodish HF. Ligandindependent oligomerization of cell-surface erythropoietin receptor is mediated by the transmembrane domain. Proc Natl Acad Sci U S A. 2001 Apr 10;98(8):4379-84.

Deacon RM. Digging and marble burying in mice: simple methods for in vivo identification of biological impacts. Nat Protoc. 2006;1(1):122-4. 
Digicaylioglu M, Bichet S, Marti HH, Wenger RH, Rivas LA, Bauer C, et al. Localization of specific erythropoietin binding sites in defined areas of the mouse brain. Proc Natl Acad Sci U S A. 1995 Apr 25;92(9):3717-20.

Digicaylioglu M, Lipton SA. Erythropoietin-mediated neuroprotection involves cross-talk between Jak2 and NF-kappaB signalling cascades. Nature. 2001 Aug 9;412(6847):641-7.

Ehrenreich H, Bartels C, Sargin D, Stawicki S, Krampe H. Recombinant human erythropoietin in the treatment of human brain disease: focus on cognition. J Ren Nutr. 2008 Jan;18(1):146-53.

Ehrenreich H, Degner D, Meller J, Brines M, Behe M, Hasselblatt M, et al. Erythropoietin: a candidate compound for neuroprotection in schizophrenia. Mol Psychiatry. 2004 Jan;9(1):4254.

Ehrenreich H, Fischer B, Norra C, Schellenberger F, Stender N, Stiefel M, et al. Exploring recombinant human erythropoietin in chronic progressive multiple sclerosis. Brain. 2007a Oct;130(Pt 10):2577-88.

Ehrenreich H, Hasselblatt M, Knerlich F, von Ahsen N, Jacob S, Sperling S, et al. A hematopoietic growth factor, thrombopoietin, has a proapoptotic role in the brain. Proc Natl Acad Sci U S A. 2005 Jan 18;102(3):862-7.

Ehrenreich H, Hinze-Selch D, Stawicki S, Aust C, Knolle-Veentjer S, Wilms S, et al. Improvement of cognitive functions in chronic schizophrenic patients by recombinant human erythropoietin. Mol Psychiatry. 2007b Feb;12(2):206-20.

El-Kordi A, Radyushkin K, Ehrenreich H. Erythropoietin improves operant conditioning and stability of cognitive performance in mice. BMC Biol. 2009;7:37.

Fu P, Jiang X, Arcasoy MO. Constitutively active erythropoietin receptor expression in breast cancer cells promotes cellular proliferation and migration through a MAP-kinase dependent pathway. Biochem Biophys Res Commun. 2009 Feb 13;379(3):696-701.

Grimm G, Stockenhuber F, Schneeweiss B, Madl C, Zeitlhofer J, Schneider B. Improvement of brain function in hemodialysis patients treated with erythropoietin. Kidney Int. 1990 Sep;38(3):480-6.

Hengemihle JM, Abugo O, Rifkind J, Spangler E, Danon D, Ingram DK. Chronic treatment with human recombinant erythropoietin increases hematocrit and improves water maze performance in mice. Physiol Behav. 1996 Jan;59(1):153-6.

Hepp S, Gerich FJ, Muller M. Sulfhydryl oxidation reduces hippocampal susceptibility to hypoxia-induced spreading depression by activating BK channels. J Neurophysiol. 2005 Aug;94(2):1091-103.

Humby T, Laird FM, Davies W, Wilkinson LS. Visuospatial attentional functioning in mice: interactions between cholinergic manipulations and genotype. Eur J Neurosci. 1999 Aug;11(8):2813-23. 
Humby T, Wilkinson L, Dawson G. Assaying aspects of attention and impulse control in mice using the 5-choice serial reaction time task. Curr Protoc Neurosci. 2005 May;Chapter 8:Unit 8 5H.

Jelkmann W. Effects of erythropoietin on brain function. Curr Pharm Biotechnol. 2005 Feb;6(1):65-79.

Jelkmann W. Erythropoietin: structure, control of production, and function. Physiol Rev. 1992 Apr;72(2):449-89.

Knabe W, Knerlich F, Washausen S, Kietzmann T, Siren AL, Brunnett G, et al. Expression patterns of erythropoietin and its receptor in the developing midbrain. Anat Embryol (Berl). 2004 Mar;207(6):503-12.

Knabe W, Siren AL, Ehrenreich H, Kuhn HJ. Expression patterns of erythropoietin and its receptor in the developing spinal cord and dorsal root ganglia. Anat Embryol (Berl). 2005 Oct;210(3):209-19.

Kogan JH, Frankland PW, Silva AJ. Long-term memory underlying hippocampus-dependent social recognition in mice. Hippocampus. 2000;10(1):47-56.

Kramer L, Madl C, Stockenhuber F, Yeganehfar W, Eisenhuber E, Derfler K, et al. Beneficial effect of renal transplantation on cognitive brain function. Kidney Int. 1996 Mar;49(3):833-8.

Kuroda M, Yokofujita J, Murakami K. An ultrastructural study of the neural circuit between the prefrontal cortex and the mediodorsal nucleus of the thalamus. Prog Neurobiol. 1998 Mar;54(4):417-58.

Liu C, Yu K, Shen K, Liu Z, Noguchi CT. Transgenic mice containing the human erythropoietin receptor gene exhibit correct hematopoietic and neural expression. Proc Assoc Am Physicians. 1996 Nov;108(6):449-54.

Liu ZY, Chin K, Noguchi CT. Tissue specific expression of human erythropoietin receptor in transgenic mice. Dev Biol. 1994 Nov;166(1):159-69.

Longmore GD, Pharr PN, Lodish HF. A constitutively activated erythropoietin receptor stimulates proliferation and contributes to transformation of multipotent, committed nonerythroid and erythroid progenitor cells. Mol Cell Biol. 1994 Apr;14(4):2266-77.

Masuda S, Nagao M, Takahata K, Konishi Y, Gallyas F, Jr., Tabira T, et al. Functional erythropoietin receptor of the cells with neural characteristics. Comparison with receptor properties of erythroid cells. J Biol Chem. 1993 May 25;268(15):11208-16.

Masuda S, Okano M, Yamagishi K, Nagao M, Ueda M, Sasaki R. A novel site of erythropoietin production. Oxygen-dependent production in cultured rat astrocytes. J Biol Chem. 1994 Jul 29;269(30):19488-93.

Miskowiak K, O'Sullivan U, Harmer CJ. Erythropoietin enhances hippocampal response during memory retrieval in humans. J Neurosci. 2007 Mar 14;27(11):2788-92. 
Miskowiak KW, Favaron E, Hafizi S, Inkster B, Goodwin GM, Cowen PJ, et al. Effects of erythropoietin on emotional processing biases in patients with major depression: an exploratory fMRI study. Psychopharmacology (Berl). 2009 Nov;207(1):133-42.

Morishita E, Masuda S, Nagao M, Yasuda Y, Sasaki R. Erythropoietin receptor is expressed in rat hippocampal and cerebral cortical neurons, and erythropoietin prevents in vitro glutamate-induced neuronal death. Neuroscience. 1997 Jan;76(1):105-16.

Morris R. Developments of a water-maze procedure for studying spatial learning in the rat. J Neurosci Methods. 1984 May;11(1):47-60.

Moucadel V, Constantinescu SN. Differential STAT5 signaling by ligand-dependent and constitutively active cytokine receptors. J Biol Chem. 2005 Apr 8;280(14):13364-73.

Moy SS, Nadler JJ, Perez A, Barbaro RP, Johns JM, Magnuson TR, et al. Sociability and preference for social novelty in five inbred strains: an approach to assess autistic-like behavior in mice. Genes Brain Behav. 2004 Oct;3(5):287-302.

Muller M, Somjen GG. Inhibition of major cationic inward currents prevents spreading depression-like hypoxic depolarization in rat hippocampal tissue slices. Brain Res. 1998 Nov 23;812(1-2):1-13.

Neubauer AP, Voss W, Wachtendorf M, Jungmann T. Erythropoietin improves neurodevelopmental outcome of extremely preterm infants. Ann Neurol. 2010 May;67(5):657-66.

Pickett JL, Theberge DC, Brown WS, Schweitzer SU, Nissenson AR. Normalizing hematocrit in dialysis patients improves brain function. Am J Kidney Dis. 1999 Jun;33(6):1122-30.

Robbins TW. The 5-choice serial reaction time task: behavioural pharmacology and functional neurochemistry. Psychopharmacology (Berl). 2002 Oct;163(3-4):362-80.

Rotaru DC, Barrionuevo G, Sesack SR. Mediodorsal thalamic afferents to layer III of the rat prefrontal cortex: synaptic relationships to subclasses of interneurons. J Comp Neurol. 2005 Sep 26;490(3):220-38.

Sadamoto Y, Igase K, Sakanaka M, Sato K, Otsuka H, Sakaki S, et al. Erythropoietin prevents place navigation disability and cortical infarction in rats with permanent occlusion of the middle cerebral artery. Biochem Biophys Res Commun. 1998 Dec 9;253(1):26-32.

Sargin D, Friedrichs H, El-Kordi A, Ehrenreich H. Erythropoietin as neuroprotective and neuroregenerative treatment strategy: Comprehensive overview of 12 years of preclinical and clinical research. Best Pract Res Clin Anaestesiol. 2010;(in press).

Siren AL, Fasshauer T, Bartels C, Ehrenreich H. Therapeutic potential of erythropoietin and its structural or functional variants in the nervous system. Neurotherapeutics. 2009 Jan;6(1):108-27. 
Siren AL, Fratelli M, Brines M, Goemans C, Casagrande S, Lewczuk P, et al. Erythropoietin prevents neuronal apoptosis after cerebral ischemia and metabolic stress. Proc Natl Acad Sci U S A. 2001 Mar 27;98(7):4044-9.

Takakusaki K, Saitoh K, Harada H, Kashiwayanagi M. Role of basal ganglia-brainstem pathways in the control of motor behaviors. Neurosci Res. 2004 Oct;50(2):137-51.

Uylings HB, Groenewegen HJ, Kolb B. Do rats have a prefrontal cortex? Behav Brain Res. 2003 Nov 30;146(1-2):3-17.

Watowich SS, Yoshimura A, Longmore GD, Hilton DJ, Yoshimura Y, Lodish HF. Homodimerization and constitutive activation of the erythropoietin receptor. Proc Natl Acad Sci U S A. 1992 Mar 15;89(6):2140-4.

Wrenn CC, Turchi JN, Schlosser S, Dreiling JL, Stephenson DA, Crawley JN. Performance of galanin transgenic mice in the 5-choice serial reaction time attentional task. Pharmacol Biochem Behav. 2006 Mar;83(3):428-40.

Wustenberg T, Begemann M, Bartels C, Gefeller O, Stawicki S, Hinze-Selch D, et al. Recombinant human erythropoietin delays loss of gray matter in chronic schizophrenia. Mol Psychiatry. 2010 May 18.

Yoshimura A, Longmore G, Lodish HF. Point mutation in the exoplasmic domain of the erythropoietin receptor resulting in hormone-independent activation and tumorigenicity. Nature. 1990 Dec 13;348(6302):647-9. 
Sargin et al Figure 1

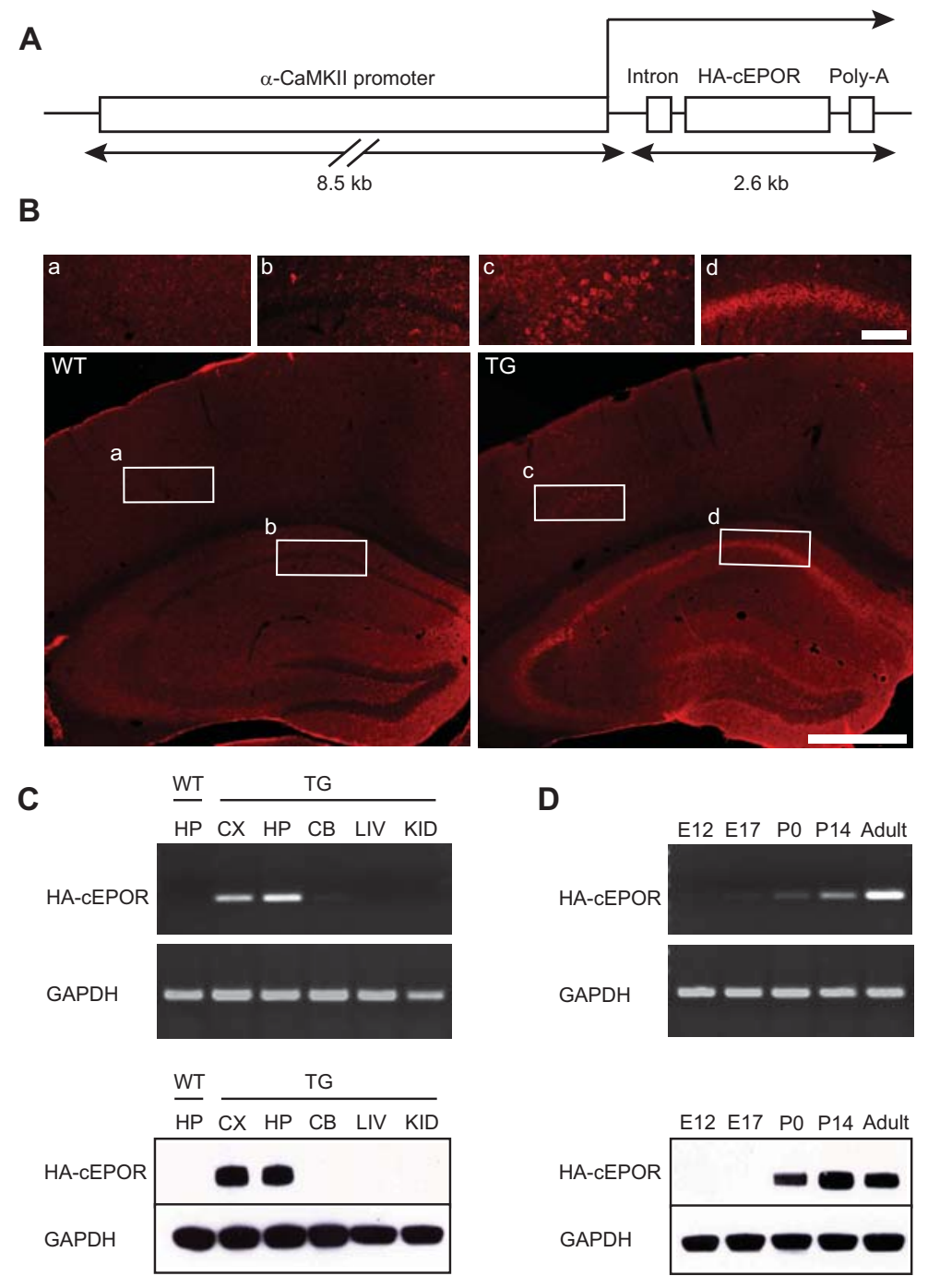


N.
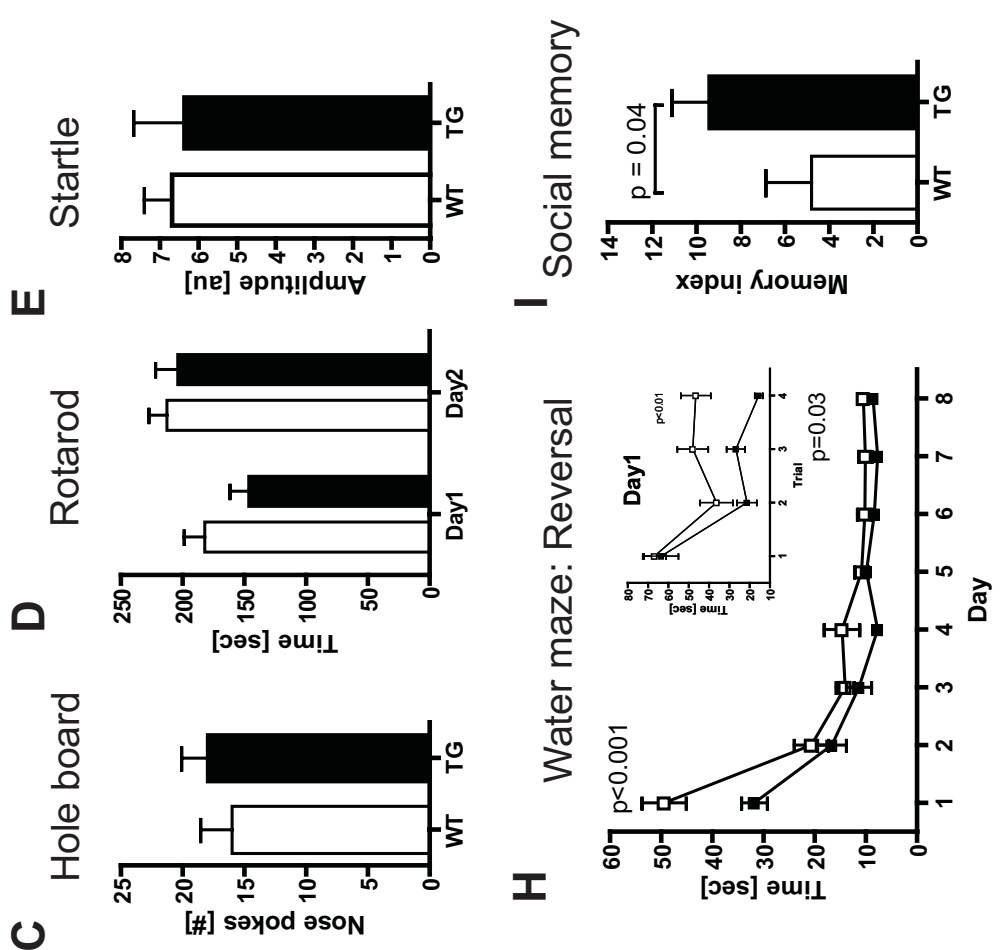

0
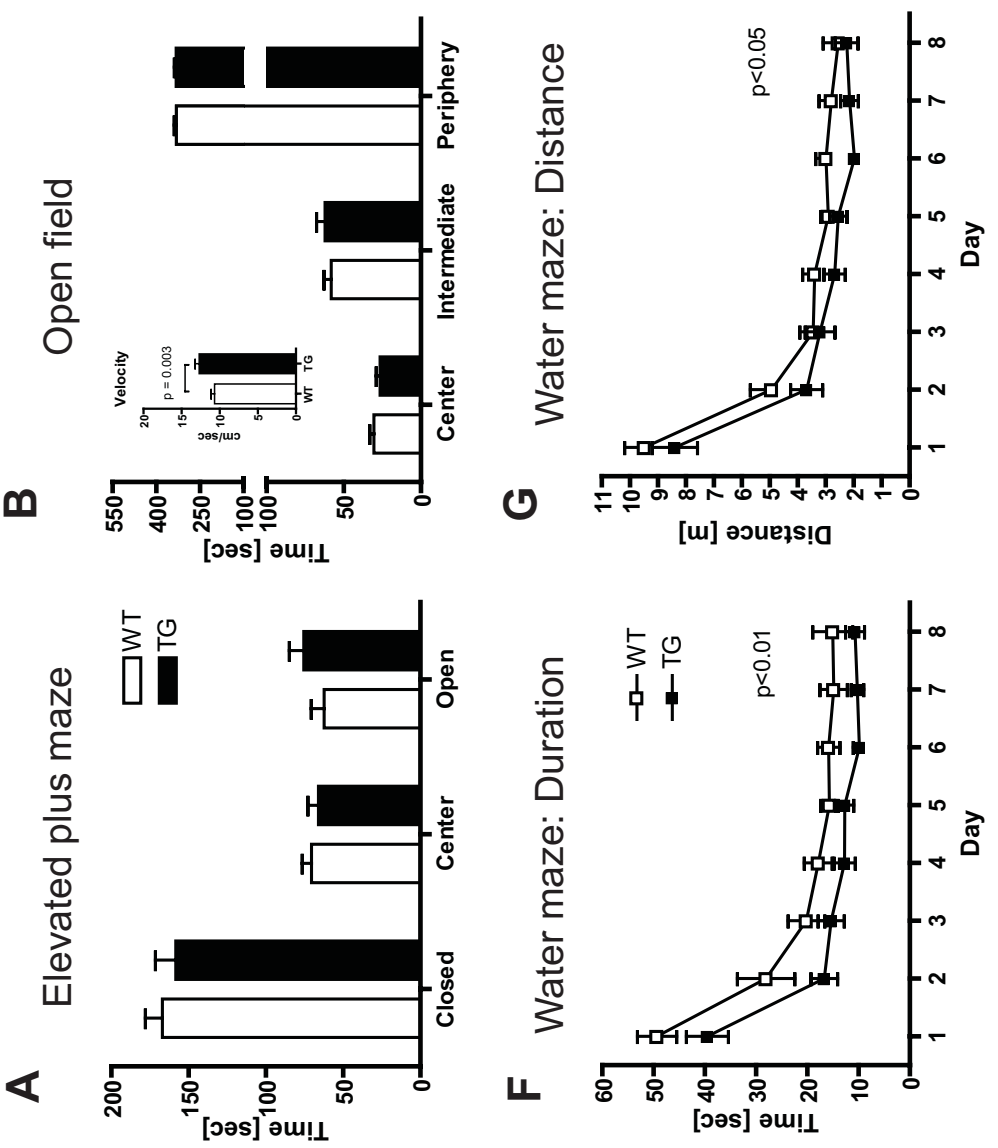


\section{Sargin et al Figure 3}

A

Paired-pulse facilitation

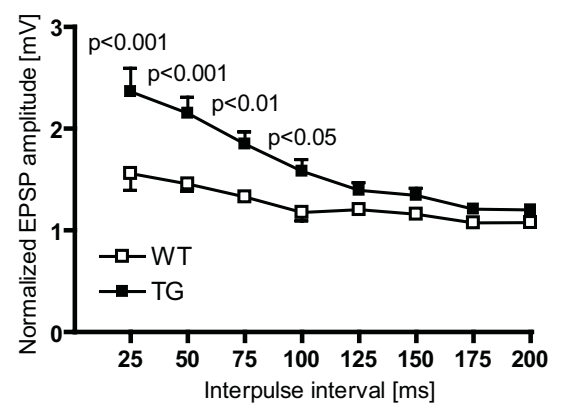

B

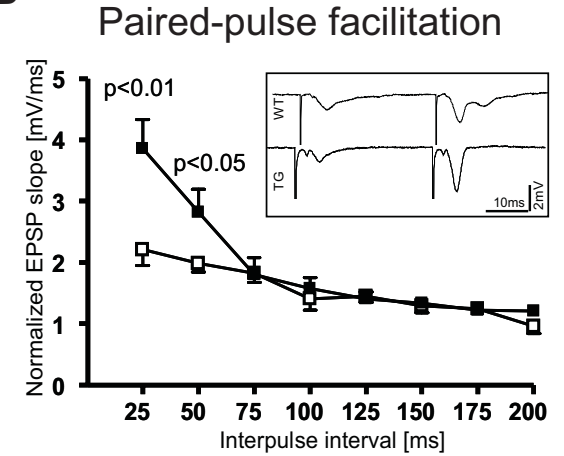

C

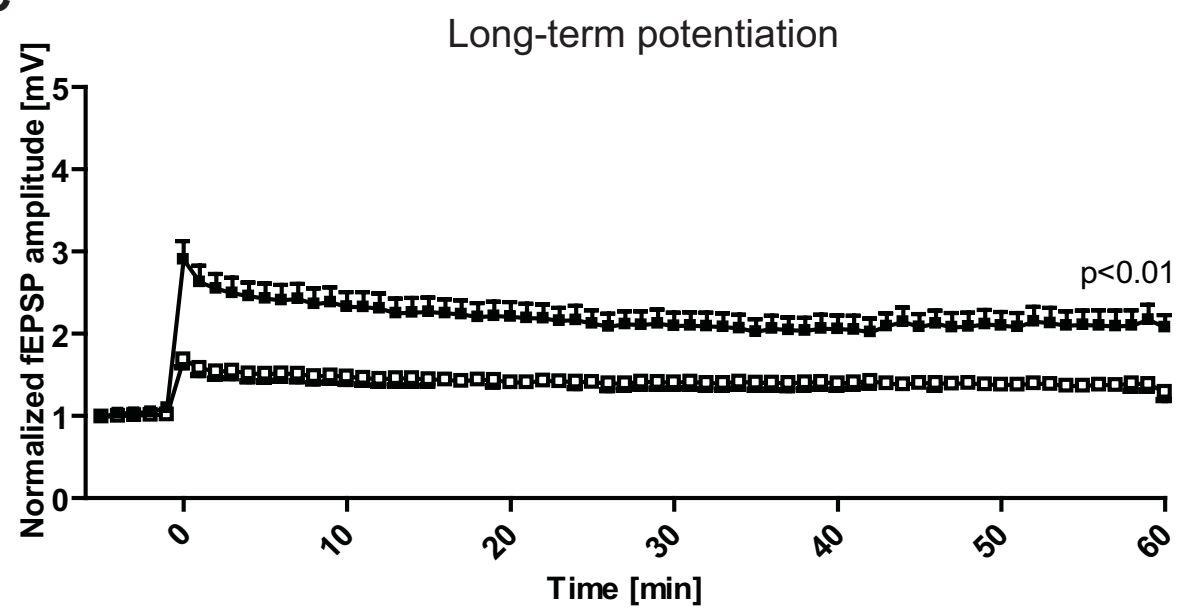

D

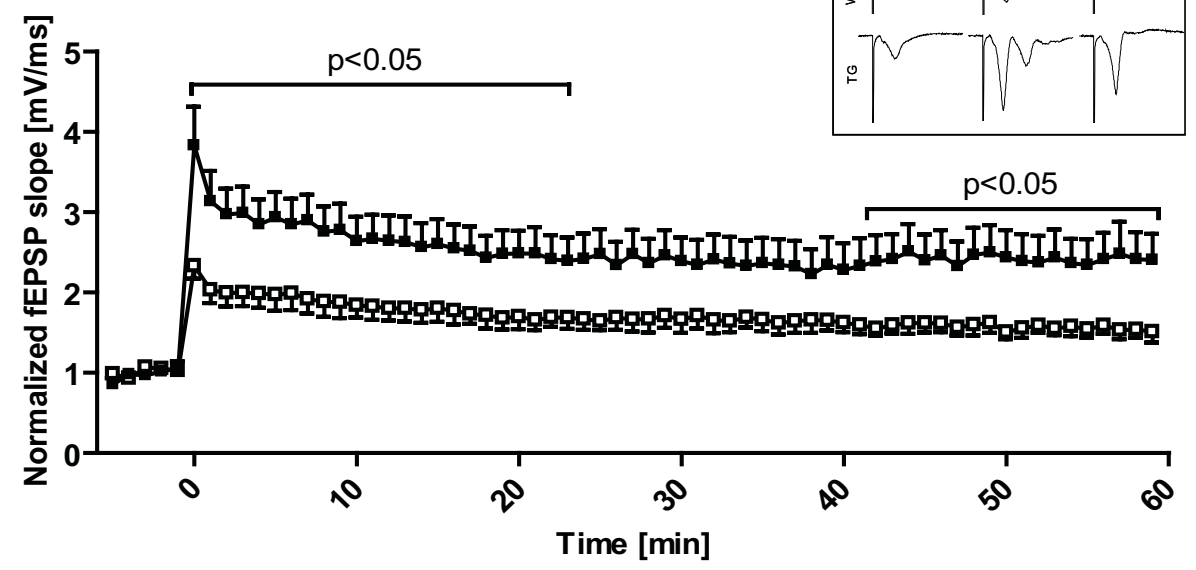



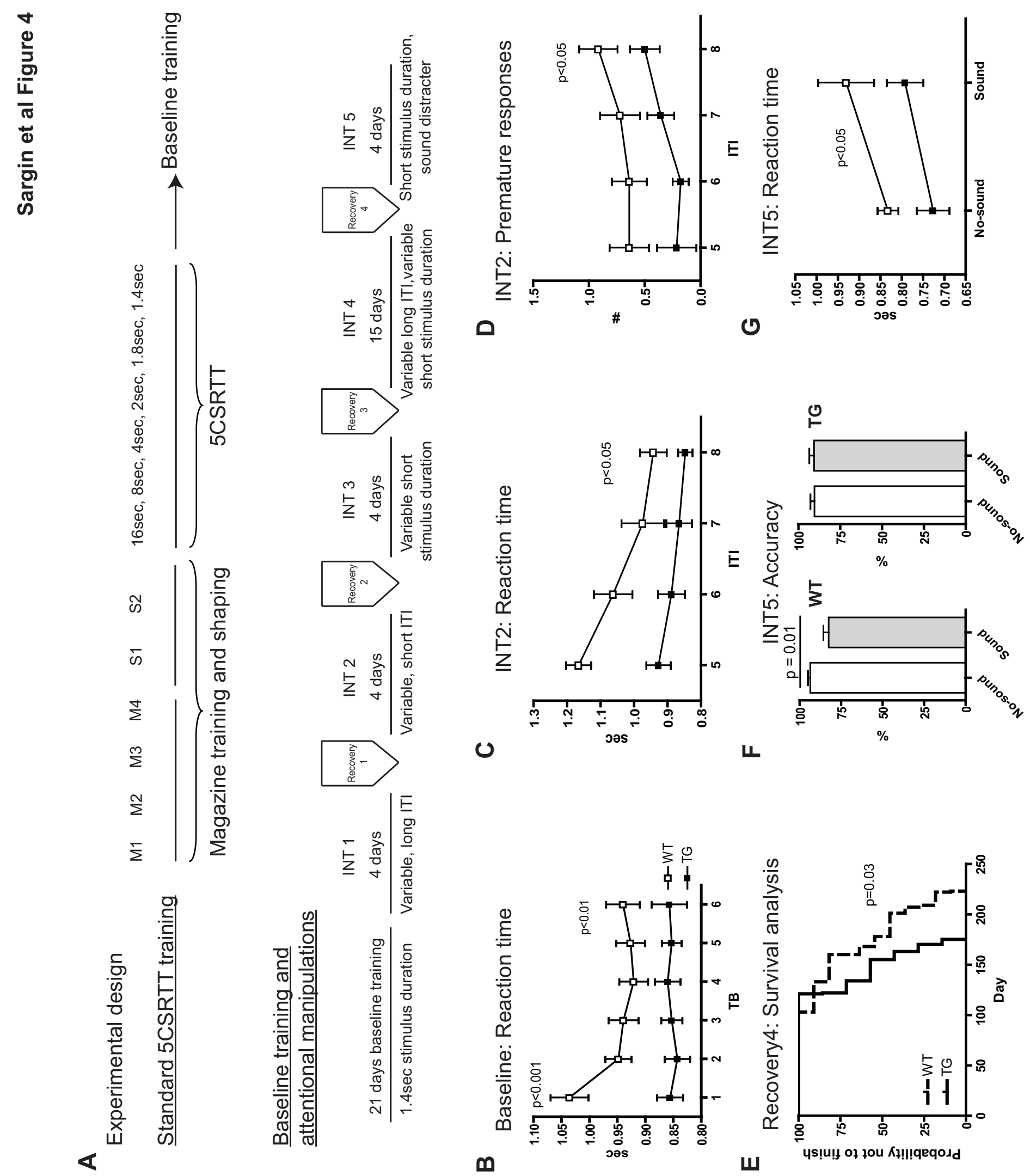

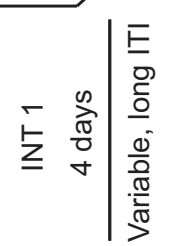
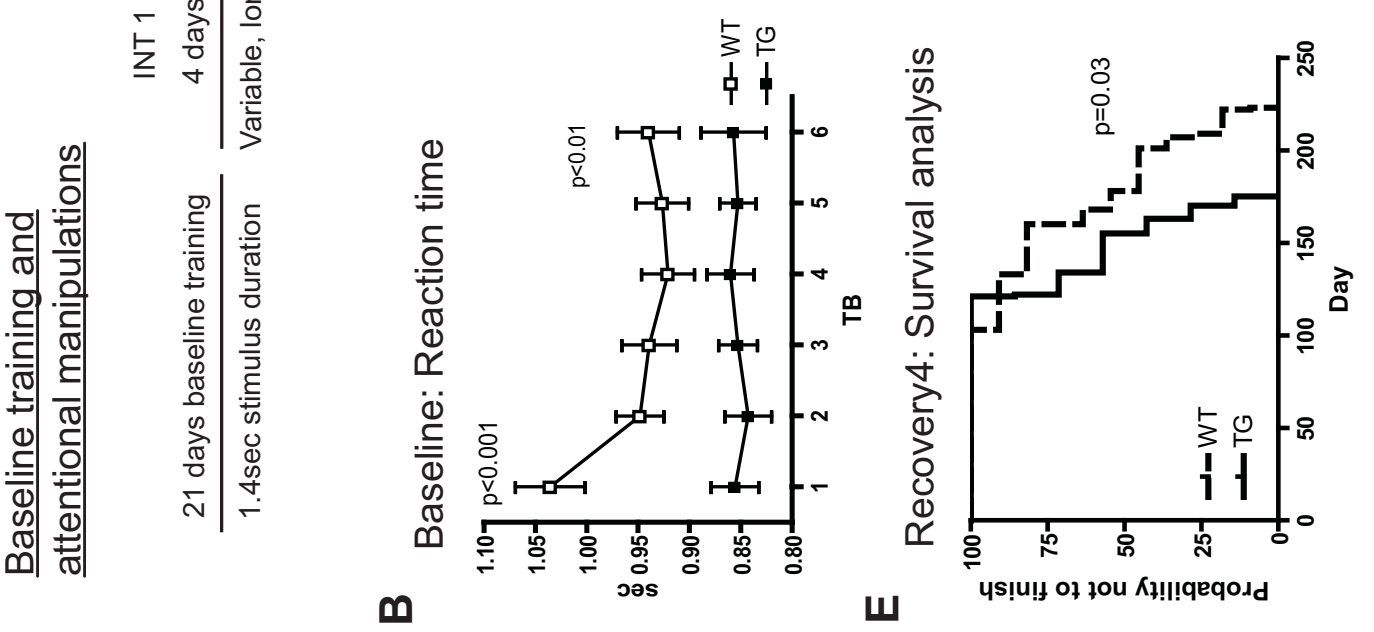
ம
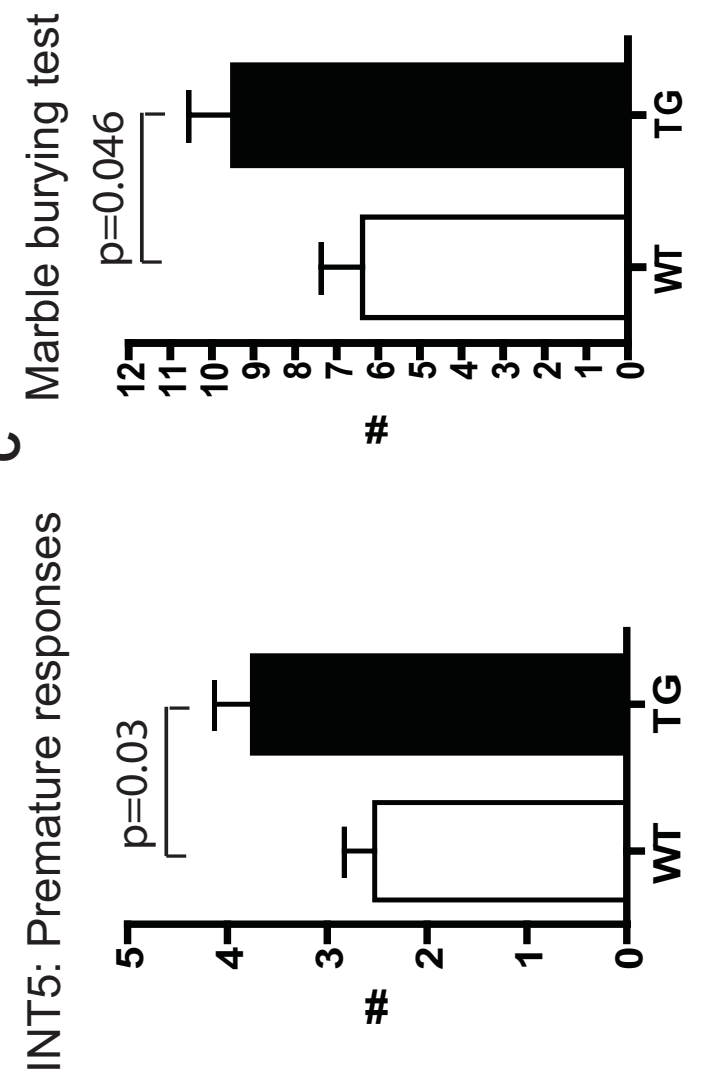

m

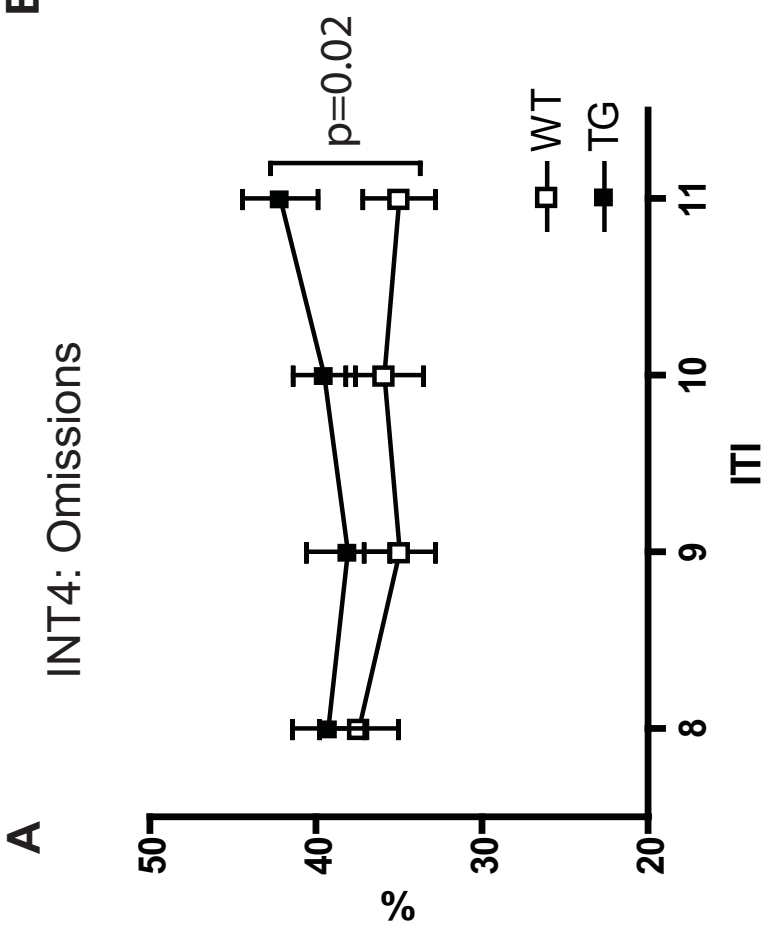


Sargin et al Supplementary Figure 1

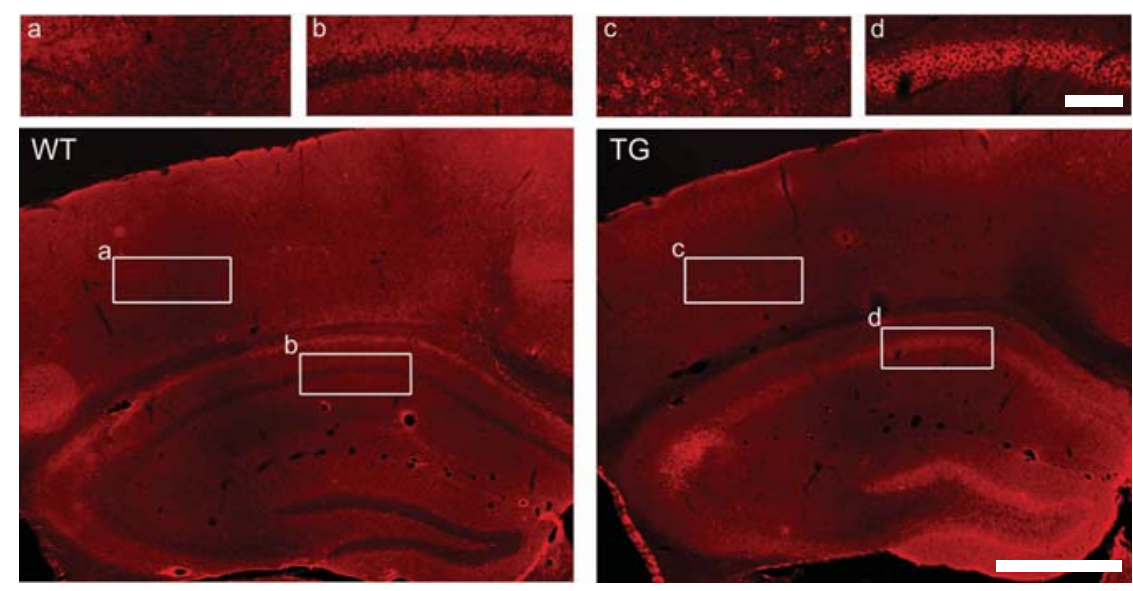


Sargin et al Supplementary Figure 2
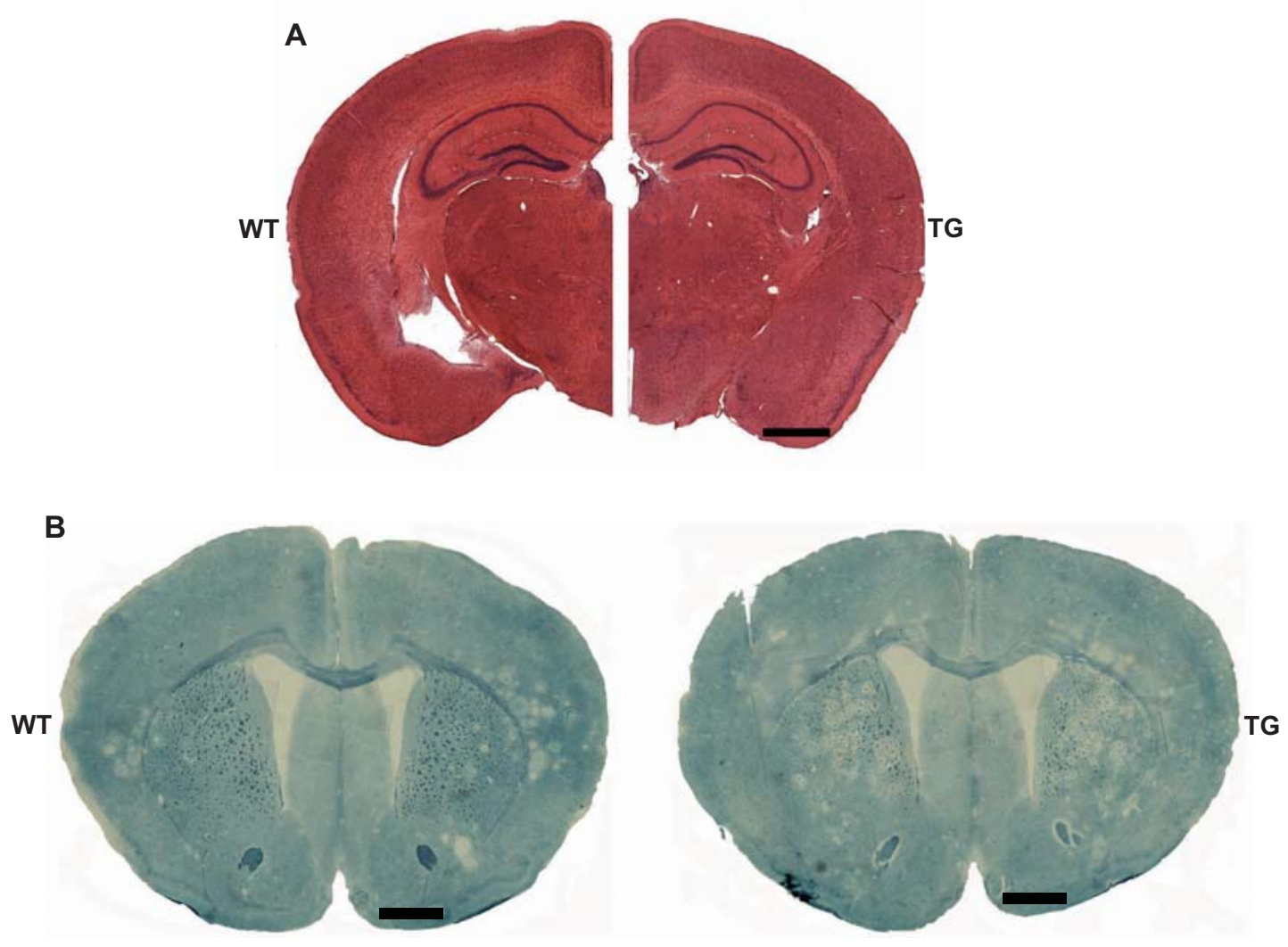


\section{SUMMARY \& CONCLUSIONS}

In the first original publication we studied the effect of combining a complexin2 null mutation (genetic predisposition) with a juvenile parietal neurotrauma as a second hit (environmental trigger) on inducing cognitive phenotypes relevant to schizophrenia in mice. Several months after lesion, only $C p / x 2$ null mutants showed reduced pre-pulse inhibition (measurement for sensory motor gating), deficit of spatial learning, and loss of inhibition after MK-801 administration. Forced alternation in T-maze, object recognition, social interaction and elevated plus maze tests were unaltered in all experimental groups. Additionally, we have replicated the previously reported mild motor phenotype of $C p / x 2$ null mutants. However, this phenotype was accentuated upon lesion. Finally, MRI volumetrical analysis revealed a decrease of hippocampal volume exclusively in lesioned Cplx2 null mutants. This goes in concert with the observed deficits in spatial learning, which rely on the hippocampus.

Taken together, the combination of $C p l \times 2$ null mutation with juvenile neurotrauma provided a further proof for the interaction between genetic predisposition and environmental triggers in the emergence of schizophrenia. This model satisfied almost all validity issues, which have to be met while modeling schizophrenia in rodents.

From there, we wanted to investigate potential treatment approaches of cognitive dysfunctions in brain diseases. Since there are no satisfying treatments for cognitive decline in schizophrenia and other neuropsychiatric diseases and due to the often reported neurodegeneration in the schizophrenic brain, we aimed at delineating the spectrum of cognitive domains potentially targeted by EPO (as a promising neuroprotective candidate). For this purpose, in the second project we made use of a sequential operant paradigm for rodents, which addresses different types of learning, attention and executive functions - the five choice serial reaction time task. We found upon 3-week EPO treatment of young healthy male mice improvements in associative, operant and discriminant learning. Moreover, EPO treated mice were faster in acquiring the task, i.e. reacting correctly to a short randomly appearing visual stimulus in one of five possible locations. Furthermore, EPO led to more stable cognitive performance. For the first time, this study showed (1) the effect of EPO on higher cognitive functions in healthy young mice, which is similar to our previous 
findings in chronic schizophrenia patients (Ehrenreich, Hinze-Selch et al., 2007) and (2) the long lasting effect of this neuroprotective compound, since the cognitive improvements were detected up to 3 months after termination of EPO treatment. This again parallels its enduring action in a neurological population (Ehrenreich, Fischer et al., 2007).

Up to that point the cellular contribution of EPO-mediated action was still obscure. For gaining more mechanistic insight on EPO action on higher cognitive functions, we designed the third study, in which we over-expressed EPO receptor (EPOR) in selected neurons. We developed a transgenic mouse model expressing EPOR under the $\alpha$-calcium/calmodulin-dependent protein kinase II ( $\alpha$-CaMKII) promoter, i.e. in pyramidal neurons of cortex and hippocampus, i.e. in brain regions heavily involved in higher cognition. Behavioral tests revealed improved spatial learning abilities, enhanced social memory and superior attentional functions of EPOR transgenic mice. Along these lines, transgenic mice had enhanced synaptic plasticity, which was indicated by increased long-term potentiation. However, perhaps as a price for higher cognition, transgenic mice had signs of reduced behavioral control under higher cognitive challenge and were mildly hyperactive in the open field. This study showed for the first time the effect of selectively stimulating EPOR in neuronal subpopulations involved in higher cognition. By circumventing the hematopoietic system this study proved that EPO-mediated cognitive improvement is due to the receptiveness of neuronal structures to EPO. In contrast to the cognitive advantages, over-stimulation of EPOR in selected brain areas led to hyperactivity and decreased behavioral control under cognitive challenge.

Taken together, the work presented in this thesis showed, that EPO is a very attractive and promising neuroprotective agent for deficits of higher cognitive functions. The findings presented in this work open new avenues for treating neuropsychiatric cognitive deficits. This has still to be addressed in the here proposed mouse model of schizophrenia. The knowledge gained here will be further pursued. We are planning to look at the effect of over-expressing EPOR in interneurons, and thus stimulating inhibitory properties of neuronal networks. 


\section{LITERATURE}

Adamcio B, Sargin D, Stradomska A, Medrihan L, Gertler C, Theis F, et al. Erythropoietin enhances hippocampal long-term potentiation and memory. BMC Biol. 2008;6:37.

American-Psychiatric-Association. Diagnostic and Statistical Manual of Mental Disorders. 4th ed. Washington, DC: American Psychiatric Association; 2000.

Ayhan Y, Sawa A, Ross CA, Pletnikov MV. Animal models of gene-environment interactions in schizophrenia. Behav Brain Res. 2009 Dec 7;204(2):274-81.

Bayer TA, Falkai P, Maier W. Genetic and non-genetic vulnerability factors in schizophrenia: the basis of the "two hit hypothesis". J Psychiatr Res. 1999 NovDec;33(6):543-8.

Blondeau C, Dellu-Hagedorn F. Dimensional analysis of ADHD subtypes in rats. Biol Psychiatry. 2007 Jun 15;61(12):1340-50.

Brines M, Cerami A. Emerging biological roles for erythropoietin in the nervous system. Nat Rev Neurosci. 2005 Jun;6(6):484-94.

Brose N. For better or for worse: complexins regulate SNARE function and vesicle fusion. Traffic. 2008 Sep;9(9):1403-13.

Buchanan RW, Freedman R, Javitt DC, Abi-Dargham A, Lieberman JA. Recent advances in the development of novel pharmacological agents for the treatment of cognitive impairments in schizophrenia. Schizophr Bull. 2007 Sep;33(5):1120-30.

Carli M, Robbins TW, Evenden JL, Everitt BJ. Effects of lesions to ascending noradrenergic neurones on performance of a 5-choice serial reaction task in rats; implications for theories of dorsal noradrenergic bundle function based on selective attention and arousal. Behav Brain Res. 1983 Sep;9(3):361-80.

Ehrenreich H, Bartels C, Sargin D, Stawicki S, Krampe H. Recombinant human erythropoietin in the treatment of human brain disease: focus on cognition. $J$ Ren Nutr. 2008 Jan;18(1):146-53.

Ehrenreich H, Fischer B, Norra C, Schellenberger F, Stender N, Stiefel M, et al. Exploring recombinant human erythropoietin in chronic progressive multiple sclerosis. Brain. 2007 Oct;130(Pt 10):2577-88.

Ehrenreich H, Hinze-Selch D, Stawicki S, Aust C, Knolle-Veentjer S, Wilms S, et al. Improvement of cognitive functions in chronic schizophrenic patients by recombinant human erythropoietin. Mol Psychiatry. 2007 Feb;12(2):206-20.

El-Kordi A, Radyushkin K, Adamcio B, Ehrenreich H. Rodent models of schizophrenia: Past, present and future. In: Granon S, editor. Endophenotypes of Psychiatric and Neurodegenerative Disorders in Rodent Models. Kerala: Signpost; 2009. p. 209-64. 
El-Kordi A, Radyushkin K, Ehrenreich H. Erythropoietin improves operant conditioning and stability of cognitive performance in mice. BMC Biol. 2009;7:37.

Elvevag B, Goldberg TE. Cognitive impairment in schizophrenia is the core of the disorder. Crit Rev Neurobiol. 2000;14(1):1-21.

Fu P, Jiang X, Arcasoy MO. Constitutively active erythropoietin receptor expression in breast cancer cells promotes cellular proliferation and migration through a MAPkinase dependent pathway. Biochem Biophys Res Commun. 2009 Feb 13;379(3):696-701.

Galletly CA, Clark CR, MacFarlane AC. Treating cognitive dysfunction in patients with schizophrenia. J Psychiatry Neurosci. 2000 Mar;25(2):117-24.

Glynn D, Bortnick RA, Morton AJ. Complexin II is essential for normal neurological function in mice. Hum Mol Genet. 2003 Oct 1;12(19):2431-48.

Glynn D, Reim K, Brose N, Morton AJ. Depletion of Complexin II does not affect disease progression in a mouse model of Huntington's disease (HD); support for role for complexin II in behavioural pathology in a mouse model of HD. Brain Res Bull. 2007 Apr 30;72(2-3):108-20.

Gottesman, II, Shields J. A critical review of recent adoption, twin, and family studies of schizophrenia: behavioral genetics perspectives. Schizophr Bull. 1976;2(3):360401.

Harrison PJ, Eastwood SL. Preferential involvement of excitatory neurons in medial temporal lobe in schizophrenia. Lancet. 1998 Nov 21;352(9141):1669-73.

Harrison PJ, Weinberger DR. Schizophrenia genes, gene expression, and neuropathology: on the matter of their convergence. Mol Psychiatry. 2005 Jan;10(1):40-68; image 5.

Harvey PD. Pharmacological cognitive enhancement in schizophrenia. Neuropsychol Rev. 2009 Sep;19(3):324-35.

Humby T, Wilkinson, L \& Dawson, G. Assaying Aspects of Attention and Impulse Control in Mice Using the 5-Choice Serial Reaction Time Task. Current protocols in neuroscience. 2005;31(Unit 8.5H).

Isles AR, Humby T, Walters E, Wilkinson LS. Common genetic effects on variation in impulsivity and activity in mice. J Neurosci. 2004 Jul 28;24(30):6733-40.

Jelkmann W. Effects of erythropoietin on brain function. Curr Pharm Biotechnol. 2005 Feb;6(1):65-79.

Jelkmann W. Erythropoietin: structure, control of production, and function. Physiol Rev. 1992 Apr;72(2):449-89. 
Juul S. Recombinant erythropoietin as a neuroprotective treatment: in vitro and in vivo models. Clin Perinatol. 2004 Mar;31(1):129-42.

Lecourtier L, Kelly $\mathrm{PH}$. Bilateral lesions of the habenula induce attentional disturbances in rats. Neuropsychopharmacology. 2005 Mar;30(3):484-96.

Lee HJ, Song JY, Kim JW, Jin SY, Hong MS, Park JK, et al. Association study of polymorphisms in synaptic vesicle-associated genes, SYN2 and CPLX2, with schizophrenia. Behav Brain Funct. 2005 Aug 31;1:15.

Lieberman JA. Is schizophrenia a neurodegenerative disorder? A clinical and neurobiological perspective. Biol Psychiatry. 1999 Sep 15;46(6):729-39.

Lipska BK. Using animal models to test a neurodevelopmental hypothesis of schizophrenia. J Psychiatry Neurosci. 2004 Jul;29(4):282-6.

Mala H, Alsina CG, Madsen KS, Sibbesen EC, Stick H, Mogensen J. Erythropoietin improves place learning in an 8-arm radial maze in fimbria-fornix transected rats. Neural Plast. 2005;12(4):329-40.

Malaspina D, Goetz RR, Friedman JH, Kaufmann CA, Faraone SV, Tsuang M, et al. Traumatic brain injury and schizophrenia in members of schizophrenia and bipolar disorder pedigrees. Am J Psychiatry. 2001 Mar;158(3):440-6.

Masuda S, Nagao M, Takahata K, Konishi Y, Gallyas F, Jr., Tabira T, et al. Functional erythropoietin receptor of the cells with neural characteristics. Comparison with receptor properties of erythroid cells. J Biol Chem. 1993 May 25;268(15):1120816.

Masuda S, Okano M, Yamagishi K, Nagao M, Ueda M, Sasaki R. A novel site of erythropoietin production. Oxygen-dependent production in cultured rat astrocytes. J Biol Chem. 1994 Jul 29;269(30):19488-93.

Maynard TM, Sikich L, Lieberman JA, LaMantia AS. Neural development, cell-cell signaling, and the "two-hit" hypothesis of schizophrenia. Schizophr Bull. $2001 ; 27(3): 457-76$.

McAllister TW. Traumatic Brain Injury and Psychosis: What Is the Connection? Semin Clin Neuropsychiatry. 1998 Jul;3(3):211-23.

Miskowiak K, O'Sullivan U, Harmer CJ. Erythropoietin enhances hippocampal response during memory retrieval in humans. J Neurosci. 2007 Mar 14;27(11):278892.

Miyamoto S, Duncan GE, Marx CE, Lieberman JA. Treatments for schizophrenia: a critical review of pharmacology and mechanisms of action of antipsychotic drugs. Mol Psychiatry. 2005 Jan;10(1):79-104.

Mogensen J, Boyd MH, Nielsen MD, Kristensen RS, Mala H. Erythropoietin improves spatial delayed alternation in a T-maze in rats subjected to ablation of the prefrontal cortex. Brain Res Bull. 2008 Sep 5;77(1):1-7. 
Mortimer AM. Novel antipsychotics in schizophrenia. Expert Opin Investig Drugs. 2004 Apr;13(4):315-29.

Moucadel V, Constantinescu SN. Differential STAT5 signaling by ligand-dependent and constitutively active cytokine receptors. J Biol Chem. 2005 Apr 8;280(14):1336473.

Radek RJ, Kohlhaas KL, Rueter LE, Mohler EG. Treating the cognitive deficits of schizophrenia with alpha4beta2 neuronal nicotinic receptor agonists. Curr Pharm Des. 2010 Jan;16(3):309-22.

Radyushkin K, El-Kordi A, Boretius S, Castaneda S, Ronnenberg A, Reim K, et al. Complexin2 null mutation requires a 'second hit' for induction of phenotypic changes relevant to schizophrenia. Genes Brain Behav. 2010 Aug 1;9(6):592-602.

Robbins TW. The 5-choice serial reaction time task: behavioural pharmacology and functional neurochemistry. Psychopharmacology (Berl). 2002 Oct;163(3-4):362-80.

Rund BR. Is schizophrenia a neurodegenerative disorder? Nord J Psychiatry. 2009;63(3):196-201.

Sachdev P, Smith JS, Cathcart S. Schizophrenia-like psychosis following traumatic brain injury: a chart-based descriptive and case-control study. Psychol Med. 2001 Feb;31(2):231-9.

Sakanaka M, Wen TC, Matsuda S, Masuda S, Morishita E, Nagao M, et al. In vivo evidence that erythropoietin protects neurons from ischemic damage. Proc Natl Acad Sci U S A. 1998 Apr 14;95(8):4635-40.

Sargin D, El-Kordi A, Agarwal A, Müller M, Wojcik SM, Hassouna I, et al. Expression of constitutively active erythropoietin receptor in cortical neurons boosts higher cognitive functions in mice. (Submitted). 2010.

Sargin D, Friedrichs H, El-Kordi A, Ehrenreich H. Erythropoietin as neuroprotective and neuroregenerative treatment strategy: Comprehensive overview of 12 years of preclinical and clinical research. Best Pract Res Clin Anaestesiol (In press). 2010.

Sargin D, Hassouna I, Sperling S, Siren AL, Ehrenreich H. Uncoupling of neurodegeneration and gliosis in a murine model of juvenile cortical lesion. Glia. 2009 May;57(7):693-702.

Sawa A, Snyder SH. Schizophrenia: diverse approaches to a complex disease. Science. 2002 Apr 26;296(5568):692-5.

Sawada K, Young CE, Barr AM, Longworth K, Takahashi S, Arango V, et al. Altered immunoreactivity of complexin protein in prefrontal cortex in severe mental illness. Mol Psychiatry. 2002;7(5):484-92. 
Simon V, van Winkel R, De Hert M. Are weight gain and metabolic side effects of atypical antipsychotics dose dependent? A literature review. J Clin Psychiatry. 2009 Jul;70(7):1041-50.

Sirén AL, Fasshauer T, Bartels C, Ehrenreich H. Therapeutic Potential of Erythropoietin and its Structural or Functional Variants in the Nervous System. Neurotherapeutics. 2009 Jan;6(1):108-27.

Sirén AL, Knerlich F, Poser W, Gleiter CH, Bruck W, Ehrenreich H. Erythropoietin and erythropoietin receptor in human ischemic/hypoxic brain. Acta Neuropathol. 2001 Mar;101(3):271-6.

Sirén AL, Radyushkin K, Boretius S, Kammer D, Riechers CC, Natt O, et al. Global brain atrophy after unilateral parietal lesion and its prevention by erythropoietin. Brain. 2006 Feb;129(Pt 2):480-9.

Stroup TS, Alves WM, Hamer RM, Lieberman JA. Clinical trials for antipsychotic drugs: design conventions, dilemmas and innovations. Nat Rev Drug Discov. 2006 Feb;5(2):133-46.

Thompson PM, Vidal C, Giedd JN, Gochman P, Blumenthal J, Nicolson R, et al. Mapping adolescent brain change reveals dynamic wave of accelerated gray matter loss in very early-onset schizophrenia. Proc Natl Acad Sci U S A. 2001 Sep 25;98(20):11650-5.

van Os J, Kapur S. Schizophrenia. Lancet. 2009 Aug 22;374(9690):635-45.

Wong $\mathrm{AH}$, Van Tol $\mathrm{HH}$. Schizophrenia: from phenomenology to neurobiology. Neurosci Biobehav Rev. 2003 May;27(3):269-306.

Wüstenberg T, Begemann M, Bartels C, Gefeller O, Stawicki S, Hinze-Selch D, et al. Recombinant human erythropoietin delays loss of gray matter in chronic schizophrenia. Mol Psychiatry. 2010 May 18.

Yamauchi Y, Qin LH, Nishihara M, Sawada K, Kato K, Inoue S. Vulnerability of synaptic plasticity in the complexin II knockout mouse to maternal deprivation stress. Brain Res. 2005 Sep 14;1056(1):59-67.

Zhang Q, Sachdev PS. Psychotic disorder and traumatic brain injury. Curr Psychiatry Rep. 2003 Jul;5(3):197-201.

Zhang Y, Xiong Y, Mahmood A, Meng Y, Qu C, Schallert T, et al. Therapeutic effects of erythropoietin on histological and functional outcomes following traumatic brain injury in rats are independent of hematocrit. Brain Res. 2009 Oct 19;1294:153-64. 


\section{LIST OF PUBLICATIONS}

Sargin D, Friedrichs H, El-Kordi A and Ehrenreich H (2010). Erythropoietin as neuroprotective and neuroregenerative treatment strategy: Comprehensive overview of 12 years of preclinical and clinical research. Best Practice \& Research: Clinical Anaesthesiology. In press.

Sargin D, El-Kordi A*, Agarwal A, Müller M, Wojcik SM, Hassouna I, Nave K-A and Ehrenreich $H$ (2010). Expression of constitutively active erythropoietin receptor in cortical neurons boosts higher cognitive functions in mice. Submitted.

${ }^{*}$ Equally contributing first author

Radyushkin K, El-Kordi A*, Boretius S, Castaneda S, Ronnenberg A, Reim K, Bickeböller H, Frahm J, Brose $\mathrm{N}$ and Ehrenreich $\mathrm{H}$ (2010). Complexin2 null mutation requires a 'second hit' for induction of phenotypic changes relevant to schizophrenia. Genes, Brain and Behavior, 9:592-602.

${ }^{*}$ Equally contributing first author

Schwab M, Gummert M, Zang M, Agarwal A, Radyushkin K, El-Kordi A, Hülsmann S, Fuchs E, Monyer H, Lai C, Ehrenreich H, Nave K-A and Zhang W (2010). ErbB4 signaling in projection neurons and parvalbumin-positive interneurons serves opposing functions in cortical inhibition. Submitted.

El-Kordi A, Radyushkin K, Adamcio B and Ehrenreich H (2009). Rodent models of schizophrenia: Past, present and future. In: Endophenotypes of Psychiatric and Neurodegenerative Disorders in Rodent Models. Ed. Granon S. Signpost.

El-Kordi A, Radyushkin K and Ehrenreich H (2009). Erythropoietin improves operant conditioning and stability of performance in mice. BMC Biology, 7:37 
Reim K, Regus-Leidig H, Ammermüller J, El-Kordi A, Radyushkin K, Ehrenreich H, Brandstätter JH and Brose $\mathrm{N}$ (2009). Aberrant function and structure of retinal ribbon synapses in the absence of complexin 3 and complexin 4. J Cell Sci. 1;102 (Pt.9), 1352-61.

Radyushkin K, Hammerschmidt K, Boretius S, Varoqueaux F, El-Kordi A, Ronnenberg A, Winter D, Frahm J, Fischer J, Brose N and Ehrenreich H. (2009). Neuroligin-3 deficient mice: Model of a monogenic heritable form of autism with an olfactory deficit. Genes Brain Behav, Jun;8(4):416-25.

Adamcio B, Sargin D, Stradomska A, Medrihan L, Gertler C, Theis F, Zhang M, Müller M, Hassouna I, Hannke K, Sperling S, Radyushkin K, El-Kordi A, Schulze L, Ronnenberg A, Wolf F, Brose N, Rhee JS, Zhang W and Ehrenreich H. (2008). Erythropoietin enhances hippocampal long-term potentiation and memory. BMC Biol, 6:37.

Krampe H, Stawicki S, Wagner, T, Ribbe K, El-Kordi A, Niehaus S, Timner W and Ehrenreich H (2005). ALITA - Ambulante Langzeit-Intensivtherapie für Alkoholkranke [OLITA - Outpatient Longterm Intensive Therapy for Alcoholics]. Psychoneuro, 31 (10): 507-512. 


\section{CURRICULUM VITAE}

\section{Personal data}

Date of birth:

Place of birth:

Nationality:

\section{Education}

from March 2007

February 2007

May 2002
16.12.1982

Cairo, Egypt

Egyptian
PhD student at the Division of Clinical Neuroscience (Head: Prof. Dr. Dr. H. Ehrenreich) at the Max Planck Institute of Experimental Medicine, Göttingen.

Diploma in Psychology, Georg-August-University Göttingen (Thesis title: The establishment and modification of the Five-Choice Serial Reaction Time Task (5-CSRTT) for modelling higher brain functions in mice), final grade: very good.

German "Abitur" at the German School (Deutsche Evangelische Oberschule) in Cairo, Egypt

\section{Work and practical experience}

October 04 - February $07 \quad$ Student research assistant at the Division of Clinical Neuroscience (Prof. H. Ehrenreich), Max Planck Institute of Experimental Medicine, Göttingen.

March 04 - September 04

Student research assistant at the Department of Cognitive and Decision Sciences (Prof. M. Waldmann), Georg-Elias-Müller Institute of Psychology, University of Göttingen.

Winter term 03/04

Conduction of tutorial in statistics and research methods in psychology until summer term 2004.

October 03 - March 04

Student research assistant at the Department of Work and Cognitive Psychology (Prof. G. Lüer), Georg-EliasMüller Institute of Psychology, University of Göttingen.

December 02 - October 03 Student research assistant at the Department of Differential and Personality Psychology (Prof. S. Ertel), Georg-Elias-Müller Institute, University of Göttingen. 


\section{Research skills}

\subsection{Human neuropsychology}

Conduction and evaluation of basic neuropsychological functions, e.g. learning abilities, short- and long-term memory, attention and executive functions using established test batteries (HAWIE-R, I-S-T2000, LPS, VLMT, TAP, MMST, Tower of Hanoi, a.o.).

\subsection{Behavioural and ex vivo neuroscience}

Conduction and evaluation of basic and cognitive functions in mice using established behavioural tests (elevated plus maze, open field, hole board, rotarod, prepulse inhibition, fear conditioning, 8-arm maze, Morris water maze, assessment of social and sensory functions, e.g. pain sensation, hearing, olfaction and vision). Development and modification of new tests and test paradigms, e.g. creation of modified versions of the five-choice serial reaction time task (5-CSRTT) for comprehensive evaluation of higher brain functions in mice. Advanced knowledge on rodent brain magnetic resonance imaging (MRI), including region-specific volumetrical analysis. Analysis and processing of hippocampal electrophysiology data.

\subsection{Cellular and in vitro neuroscience}

Basic lab techniques (PCR, Western, Northern), including dissection and preparation of rodent brain tissue for biochemical and molecular biological analysis, brain perfusion.

\section{Computer and language skills}

\subsection{Computer skills}

- Office packages: Excel, Power Point, Word, Outlook

- Acrobat Photoshop

- SPSS 16

- $\quad$ Prism 4.03 for Windows

- Amira 4.1

Experimental scientific software: e-Prime, ProLab, Med. Associates, MATLAB, Clampfit

\subsection{Languages}

Arabic: $\quad$ Native language

German: $\quad$ Fluent

English: $\quad$ Fluent

French: Intermediate

\section{Teaching and supervision}

\subsection{Teaching}

Junior lecturer in Translational Neuroscience: Schizophrenia $(2008,2009,2010)$. Topics: Animal models of schizophrenia, testing brain functions in rodents, neurophysiology, neuropathology and histology, hippocampal plasticity.

Junior lecturer in Translational Neuroscience: Multiple Sclerosis (2008, 2009). Topics: Testing motor functions in rodents, neurophysiology of MS, glucocorticoids and MS. 


\subsection{Supervision}

Supervision of practical internships / lab rotations of:

Daniela Winkler, June-August 2010

Caroline Ritter, November-December 2009

Salah Abdo, Yemen, November 2009

Salvador Castaneda Vega, El Salvador, June-August 2009

Martesa Tantra, University of Osnabrück, April 2009

Derya Akad, Neuroscience Program, May 2008

Hoon Soon, Neuroscience Program, May 2008

\subsection{Reviewer}

Ad hoc reviewer (together with Prof. Ehrenreich) for the following journals Journal of Pharmacology and Experimental Therapeutics

Journal of Neuroscience

Neuroscience

European Journal of Neuroscience

Genes, Brain and Behavior

\section{Affiliations and memberships}

Associate member of the German Psychological Association, Section Biological Psychology and Neuropsychology (since 2007).

Student representative of the PhD program Systems Neuroscience in the Göttingen Graduate School for Neurosciences and Molecular Biosciences (GGNB) and Member of the Examination Board (2008-2010).

\section{Attended Conferences and Symposia}

04.-09.05.2010 Workshop on novel approaches to neuroprotection and neuroregeneration. The Hebrew University of Jerusalem, Israel.

14. -16.11 .2009$

04.11 .2009

30.07. - 01.08.2009

20.04.2009
EMBL Workshop and Conference on "Translating Behaviour: Bridging Clinical and Animal Model Research"

Poster presentation

Heidelberg, Germany

$5^{\text {th }}$ Mini-Symposium of the Center for Systems Neuroscience Organizing committee

Göttingen, Germany

$8^{\text {th }}$ International Lübeck Conference on the Pathophysiology and Pharmacology of Erythropoietin and other Hematopoietic Growth Factors

Poster presentation

Lübeck, Germany

$4^{\text {th }}$ Mini-Symposium of the Center for Systems Neuroscience

Organizing committee

Göttingen, Germany 
25. -29.03 .2009$

02. -04.10 .2008$

26. -27.09 .2008$

$29.03-01.04 .2007$

15. -16.07 .2005$

14. -15.09 .2006$

18. -19.09 .2008$

\section{Scientific Talks}

06.05 .2010

13.08.2009

06.06.2009

27.02.2009 $32^{\text {nd }}$ Göttingen Neurobiology Conference

Attended

Göttingen, Germany

Intensive workshop neuroanatomy

Attended

München, Germany

$4^{\text {th }}$ Brainstorming Conference

Poster presentation

Hannover, Germany

$31^{\text {st }}$ Göttingen Neurobiology Conference

Attended

Göttingen, Germany

International Symposia on Schizophrenia of the Göttingen

Research Association for Schizophrenia (GRAS)

Organizing committee

Göttingen, Germany

The effects of EPO on higher cognitive processes in mice Workshop on novel approaches to neuroprotection and neuroregeneration. Jerusalem, Israel

Erythropoietin improves operant conditioning and stability of cognitive performance in mice

Ahmed El-Kordi

Summer Symposium of the MPI of Experimental Medicine Göttingen, Germany

Complexin2 is a modifier gene of cognition in schizophrenia

Sergi Papiol and Ahmed El-Kordi

Retreat of the DFG Research Center Molecular Physiology of the Brain (CMPB), Bad Salzdetfurth, Germany

Rett syndrome - Of Mice and Girls

Ahmed El-Kordi

Retreat of Section B2 "From synaptopathies to system dysfunctions" of the DFG Research Center Molecular Physiology of the Brain (CMPB) Seeburger See, Germany 